\title{
STRESS ENGINEERING FOR POLARIZATION CONTROL IN SILICON-ON-INSULATOR WAVEGUIDES AND ITS APPLICATIONS IN NOVEL PASSIVE POLARIZATION SPLITTERS/FILTERS
}

\author{
by \\ Winnie Ning Ye \\ B.Eng (Carleton), M.A.Sc. (Toronto)
}

\begin{abstract}
A Thesis
Submitted to the Faculty of Graduate Studies and Research in Partial Fulfillment of the Requirements for the Degree of Doctor of Philosophy
\end{abstract}

Ottawa-Carleton Institute for Electrical Engineering Department of Electronics

Faculty of Engineering

Carleton University

Ottawa, Canada

Copyright @ December 2006 by Winnie N. Ye 


$\begin{array}{ll}\begin{array}{l}\text { Library and } \\ \text { Archives Canada }\end{array} & \begin{array}{l}\text { Bibliothèque et } \\ \text { Archives Canada }\end{array} \\ \begin{array}{l}\text { Published Heritage } \\ \text { Branch }\end{array} & \begin{array}{l}\text { Direction du } \\ \text { Patrimoine de l'édition }\end{array} \\ \begin{array}{l}\text { 395 Wellington Street } \\ \text { Ottawa ON K1A 0N4 }\end{array} & \begin{array}{l}\text { 395, rue Wellington } \\ \text { Ottana ON K1A ON4 } \\ \text { Canada Oa }\end{array}\end{array}$

Your file Votre référence ISBN: 978-0-494-23305-4 Ourfile Notre référence ISBN: $978-0-494-23305-4$

NOTICE:

The author has granted a nonexclusive license allowing Library and Archives Canada to reproduce, publish, archive, preserve, conserve, communicate to the public by telecommunication or on the Internet, loan, distribute and sell theses worldwide, for commercial or noncommercial purposes, in microform, paper, electronic and/or any other formats.

The author retains copyright ownership and moral rights in this thesis. Neither the thesis nor substantial extracts from it may be printed or otherwise reproduced without the author's permission.
AVIS:

L'auteur a accordé une licence non exclusive permettant à la Bibliothèque et Archives Canada de reproduire, publier, archiver, sauvegarder, conserver, transmettre au public par télécommunication ou par l'Internet, prêter, distribuer et vendre des thèses partout dans le monde, à des fins commerciales ou autres, sur support microforme, papier, électronique et/ou autres formats.

L'auteur conserve la propriété du droit d'auteur et des droits moraux qui protège cette thèse. $\mathrm{Ni}$ la thèse ni des extraits substantiels de celle-ci ne doivent être imprimés ou autrement reproduits sans son autorisation.
In compliance with the Canadian

Privacy Act some supporting forms may have been removed from this thesis.

While these forms may be included in the document page count, their removal does not represent any loss of content from the thesis.
Conformément à la loi canadienne sur la protection de la vie privée, quelques formulaires secondaires ont été enlevés de cette thèse.

Bien que ces formulaires aient inclus dans la pagination, il n'y aura aucun contenu manquant. 


\title{
Abstract \\ STRESS ENGINEERING FOR POLARIZATION CONTROL IN SILICON-ON-INSULATOR WAVEGUIDES AND ITS APPLICATIONS IN NOVEL PASSIVE POLARIZATION SPLITTERS/FILTERS
}

\author{
Winnie Ning Ye \\ Submitted to the Faculty of Graduate Studies and Research \\ in partial fulfillment of the requirements for the Degree of \\ Doctor of Philosophy in Electrical Engineering \\ Carleton University \\ 2006
}

Over the last decade, devices in silicon-on-insulator (SOI) have gained tremendous attention due to their potential for making highly compact monolithic integrated optoelectronic circuits. As the waveguide cross-section reduces to less than the wavelength of light, polarization control becomes increasingly challenging in the device design and operation and is one of the main obstacles to the development of viable microphotonic commercial products. This thesis explores and develops a method of polarization control in SOI: stress engineering. A systematic and comprehensive study of the geometrical and stress-induced effects on waveguide modal birefringence is presented. A new calculation scheme, the Normalized Plane-Strain Model, is proposed and implemented for stress analysis with excellent accuracy and efficiency. Both calculations and experiments confirm that the cladding induced stress can effectively modify or eliminate polarization dispersion in SOI waveguides of arbitrary shapes, for typical $\mathrm{SiO}_{2}$ cladding film stress $\left(\sigma_{\text {film }}=-100\right.$ to $\left.-300 \mathrm{MPa}\right)$ and cladding thicknesses on the order of $1 \mu \mathrm{m}$ or less.

An important application of stress engineering is in passive polarization splitters/filters. The modeling, fabrication and characterization of several novel polarization splitters and filters in the SOI platform by employing stress engineering are presented. The designs are 
mainly focused on two configurations: the Mach-Zehnder interferometer (MZI) and the zero-order arrayed waveguide grating (AWG). The main advantage of these devices is the simplicity in polarization control - only one single postfabrication step is required. In addition, stress engineering provides the freedom to decouple the birefringence constraints from the waveguide cross-section design such that the waveguides can be independently optimized for its insertion loss, coupler performance and bend loss. Furthermore, the wavelength independent characteristics of stress engineering ensure the broadband performance of the proposed devices. Functioning stress-induced polarization splitters are demonstrated experimentally in both the MZI- and zero-order AWG-based devices, exhibiting broadband polarization splitting as high as $-14 \mathrm{~dB}$ for both output polarizations from 1460 to $1570 \mathrm{~nm}$. The device sizes are $2.5 \mathrm{~mm} \times 16 \mu \mathrm{m}$ and $12 \mathrm{~mm} \times 4 \mathrm{~mm}$ for the MZI- and the zero-order AWG-based devices, respectively. This work represents the first reported use of stress engineering for making SOI polarization splitters/filters. 


\section{Acknowledgements}

I have absolutely no doubt that doing a Ph.D. degree was one of the best decisions of my life. This four-year-long journey has not always been smooth and easy. A mixture of happiness, sadness, excitement and stress, made this an unforgettable adventure. There are so many people I am grateful for who have made this journey easier and more enjoyable for me. I am glad that I have now the opportunity to express my sincere gratitude to all of them.

I would like to express my most sincere gratitude to my supervisors, Dr. Dan-Xia $\mathrm{Xu}$, Dr. Siegfried Janz and Prof. Garry Tarr, for their inspiration, guidance and support throughout the entire project. Their patience, enthusiasm, immense knowledge and encouragements have carried me through to the end of the journey. Thank you for being the greatest mentors. This dissertation would not have been possible without them.

I am grateful to my thesis committee members: Prof. Jacques Albert, Prof. Xiaoyi Bao, Prof. Robert Gauthier, Prof. Andy Knights, Prof. Tom Smy and Prof. Barry Syrett for their constructive feedback contributed to this dissertation.

I would like to take this opportunity to acknowledge all the members of the Optoelectronics and Nanofabrication group at the National Research Council for their support. A big thank-you goes to Dr. Phil Waldron for his long hours of hard work in fabrication - this dissertation would NOT have been complete without his great work. Special thanks are due to Dr. Juan Caballero for the oxide deposition, Dr. Andre Delâge for his excellent lectures in finite element method, Dr. Pavel Cheben for his advice in the device design, Dr. Adam Densmore for his support and valuable advice, Ms. Edith Post for her help in the experimental setup, Mr. Jeff Fraser for the SEM images of the fabricated devices, Mr. Hassan Zeindeddine for the computer support, and Dr. Boris Lamontagne, Dr. Pedro Barriors, Dr. Margaret Buchanan, Mr. Mark Malloy, Ms. Marie-Josée Picard, and Dr. Greg Pakulski, for their help at various stages of my research. From Carleton University, I'm deeply indebted to Mr. Nagui Mikhail for his help in fixing and setting 
up my computer, Scott Bruce and Jacques Lemieux for computer help, Chris Raum and Khaled Mnaymneh for numerous fruitful discussions, and Peggy Piccolo, Betty Zahalan, Lorena Duncan and Blazenka Power for their help in the thesis submission.

I am deeply indebted to Prof. Yasuhiko Arakawa for accepting me as a visiting research fellow at the University of Tokyo during the summer of 2005. It was a privilege to work with Arakawa Sensei and Dr. Masahiro Nomura. Fabricating photonic crystals during a 6.1 magnitude earthquake is one of my most memorable experience. A big thank-you goes to all the members of the Arakawa laboratory, James Sims, Micheal Tsay, and my close Japanese friends Masahiro Nomura, Mio Ono, and Rieko Yamada. I would like to thank my host family in Japan, the Watanabes, (Kazumi, Kimikatsu, Yoshino, Aritomo, Yuuka and Kai) for treating me as a part of their family.

I want to express my heartfelt gratitude to my good friends who have provided support and encouragement during my most stressful time. My special thanks go to Kuan Pei Yap, Peter Clipsham, Daniel-Steve Fournier, Kevin Lee, Ksenia Yadav, Mariève Chabot, Khen Tea, Erin Doherty, Simei Xiang, Jeannine Casselman, Cathy Ma, Nika Robinson, Pia Sindile and Siwei Gao. Thank you Kevin for proofreading parts of this manuscript. It has been my greatest pleasure to have friends like you! Thank you!

I believe I owe my deepest thanks to my family for their love and support throughout my life. Thank you for always believing in me and encouraging me to pursue my dreams. Mom and dad, your unconditional love has helped me through so many tough times and has made me the person I am today. 爸爸媽媽, 真心的謝謝您們. Lei, thank you for being a wonderful loving and caring sister. And Susan, thank you for always being there for me as a sister, a mentor and a close friend.

Finally, I acknowledge NSERC, CIPI, Carleton University, and the National Research Council Canada for the financial support during this work.

\section{書山有路勤鸾徑，學海無涯苦作舟。}




\section{Contents}

1 Introduction 1

1.1 Silicon-on-insulator $(\mathrm{SOI}) \ldots \ldots \ldots \ldots \ldots$

1.1.1 Fabrication of SOI Wafers ................ 3

1.1 .2 SOI Waveguides . . . . . . . . . . . . 6

1.2 Waveguide Mode and Polarization . . . . . . . . . . . 8

1.2.1 Waveguide Modal Analysis . . . . . . . . . . . . . 8

1.2 .2 Effective Index Method . . . . . . . . . . . . . . . . . 9

1.2.3 Finite Element Method . . . . . . . . . . . . . . . 11

1.2 .4 Single Mode Condition . . . . . . . . . . . . . . . . . 13

1.2.5 Waveguide Birefringence . . . . . . . . . . . . . 16

1.3 Thesis Organization . . . . . . . . . . . . . . . . . . 19

2 Literature Review and Thesis Objective 21

2.1 Past Research . . . . . . . . . . . . . . . . . . . . 21

2.1.1 Birefringence Elimination . . . . . . . . . . . 22

2.1 .2 Polarization Splitting . . . . . . . . . . . . . . . 41

2.2 Thesis Objective . . . . . . . . . . . . . . . 48

3 Stress: Theory and Modeling $\quad 50$

3.1 Waveguide Stress and Strain: Theory . . . . . . . . . . . . 52

3.2 Stress Modeling . . . . . . . . . . . . . . . . 56

vi 
3.2.1 Numerical Simulation Tool . . . . . . . . . . . . . . . . 56

3.2.2 Generalized Plane Strain Model . . . . . . . . . . . . . . . . . 57

3.2.3 Ordinary Plane Strain Model . . . . . . . . . . . . . 59

3.2.4 Normalized Plane Strain Model . . . . . . . . . . . . . . . 60

3.2.5 Stress Relief and Calculation Window Size . . . . . . . . . . 63

3.3 Summary . . . . . . . . . . . . . . . . . . . 69

4 Stress Engineering - Stress and Geometry Induced Effects $\quad 70$

4.1 Influence of Geometrical Parameters . . . . . . . . . . . . . 70

4.1 .1 Effects of Upper Oxide Cladding . . . . . . . . . . . . 71

4.1.2 Effects of Ridge Geometrical Profile . . . . . . . . . . . . . 73

4.1 .3 Effects of Ridge Height . . . . . . . . . . . . . . . . 80

4.1 .4 Summary .......................... 81

4.2 Stress-induced Birefringence . . . . . . . . . . . . . . . . 81

4.2.1 Effects of Oxide Cladding Stress . . . . . . . . . . . . . 83

4.2 .2 Effects of Ridge Etch Depth with Stress . . . . . . . . . . 88

4.2.3 Effects of Ridge Width with Stress . . . . . . . . . . . . . 90

4.2.4 Effects of Ridge Height with Stress . . . . . . . . . . . . . . 93

4.2.5 Effects of Stress in Photonic Wire Waveguides . . . . . . . . . . . 94

4.2 .6 Summary . . . . . . . . . . . . . . . . . . 95

5 Experimental Demonstration of Stress Engineering $\quad 97$

5.1 Test Arrayed Waveguide Grating Devices . . . . . . . . . . . . . . 97

5.2 Experimental Setup . . . . . . . . . . . . . . . . 101

5.3 Results . . . . . . . . . . . . . . . . . . . . 103

5.3.1 Polarization Tuning Using Stress . . . . . . . . . . . . . . . 103

5.3.2 Polarization-independent Operation Using Stress . . . . . . . . 106

5.4 Summary . . . . . . . . . . . . . . . . . . 107

vii 
6.1 Introduction . . . . . . . . . . . . . . . . 109

6.2 Splitter/Filter Operating Principles . . . . . . . . . . . . . . . 111

6.2.1 MZI with Two Y-junctions . . . . . . . . . . . . 111

6.2.2 MZI with One Y-junction and One Directional Coupler . . . . . . 113

6.2.3 MZI with One Y-junction and One MMI Coupler . . . . . . . . 116

6.2.4 MZI with Two Symmetric Directional Couplers . . . . . . . . . 118

6.2.5 MZI with Two Symmetric MMI Couplers . . . . . . . . . . . 120

6.3 Design Considerations . . . . . . . . . . . . . . . . . 122

6.3.1 Waveguide Cross-section . . . . . . . . . . . . . . 122

$6.3 .23 \mathrm{~dB}$ Couplers . . . . . . . . . . . . . . . . . 124

6.4 Simulation Results . . . . . . . . . . . . . . . . . 126

6.5 Photomask Layout . . . . . . . . . . . . . . . . . . . 130

6.6 Fabrication . . . . . . . . . . . . . . . 132

6.7 Optical Measurements . . . . . . . . . . . . . . . . 134

6.7 .1 Experimental Setup . . . . . . . . . . . . 134

6.7 .2 Test Results . . . . . . . . . . . . . . . 136

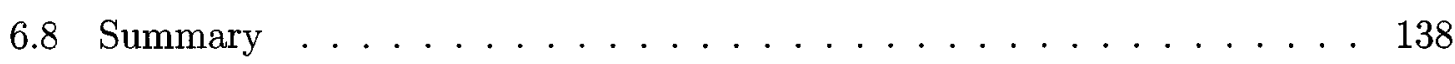

7 Zero-order Arrayed Waveguide Grating Based Polarization Splitters 140

7.1 Introduction . . . . . . . . . . . . . . . . 140

7.2 Splitter Operating Principles . . . . . . . . . . . . . . . . 142

7.3 Design Considerations . . . . . . . . . . . . . . . . 145

7.4 Simulation Results . . . . . . . . . . . . . . . . . . 148

7.5 Photomask Layout . . . . . . . . . . . . . . . . . 151

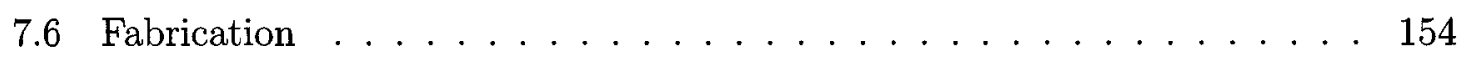

7.7 Optical Measurements . . . . . . . . . . . . 155

7.8 Summary . . . . . . . . . . . . . . . . 160

viii 
8 Conclusions

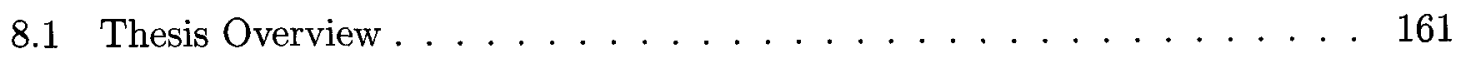

8.2 Original Contributions . . . . . . . . . . . . . 162

8.3 Significance of Work . . . . . . . . . . . . . . . . 163

8.4 Final Remarks . . . . . . . . . . . . . . . . . 168

A Stress And Modal Analysis Using FEMLAB ${ }^{\circledR} \quad 169$

B Zero-order AWG Layout Generation $\quad 176$

B.1 MATLAB ${ }^{\circledR}$ Programming Code . . . . . . . . . . . . . . . . . 178

B.2 Visual Basic ${ }^{\circledR}$ Programming Code . . . . . . . . . . . . . . . 181

$\begin{array}{ll}\text { Bibliography } & 194\end{array}$ 


\section{List of Tables}

3.1 Material parameters of $\mathrm{Si}$ (substrate and waveguide core) and $\mathrm{SiO}_{2}$ (upper and lower claddings). . . . . . . . . . . . . . . . . . . 54

3.2 Comparison of oxide film stress $\sigma_{\text {film }}$ and waveguide birefringence calculations from generalized, ordinary, and normalized plane strain models. The stress values are taken at the middle of the oxide film, $4 \mu \mathrm{m}$ away from the center of the ridge waveguide. The SOI waveguide has the following dimensions: $H=2.2 \mu \mathrm{m}, W=1.8 \mu \mathrm{m}$, and $t=1 \mu \mathrm{m}$. The stress calculation window is $100 \mu \mathrm{m}$ ( wide) $\times 100 \mu \mathrm{m}$ thick (see Fig. 3.2). Material parameters listed in Table 3.1 were used for these calculations. . . . . . .

3.3 Comparison of oxide film stress $\sigma_{\text {film }}$ and waveguide birefringence calculations from generalized and normalized plane strain models. The stress values are taken at the middle of the oxide film, $4 \mu \mathrm{m}$ away from the center of the ridge waveguide. Young's modulus of the substrate was set to $E_{s}^{\prime}=10^{3} E_{s}$. The SOI ridge waveguide has the same dimensions as in Table 3.2. The stress calculation window is reduced to $40 \mu \mathrm{m}$ wide by $5 \mu \mathrm{m}$ thick (from $100 \mu \mathrm{m} \times 100 \mu \mathrm{m}$ as in Table 3.2 ). . . . . . . . .

5.1 List of measured ridge dimensions. The targeted etch depth is $1.5 \mu \mathrm{m}$ for both the dry- and wet-etched waveguides. . . . . . . . . . . . 100 
6.1 Design parameters for the 3dB input and output couplers used in MZIbased polarization splitters and filters. . . . . . . . . . . 126

6.2 A list of device design parameters and calculated splitting ratio of the MZI-based polarization splitters and filters. . . . . . . . . . . . . . . 129

7.1 A list of design parameters for the zero-order AWGs. The spatial separation $d_{s}$ are the distance measured from the reference line, as shown in Fig. 7.5. The etch depth of all waveguides are $D=1.5 \mu \mathrm{m}$. . . . . . . 151

7.2 The measured spatial separations between the TE and TM polarizations in the devices presented in Figs. 7.14(a), 7.14(b), and 7.15 . . . . . 158 


\section{List of Figures}

1.1 Cross-section of an SOI wafer: a slab waveguide. . . . . . . . . . 3

1.2 Schematic of fabrication process flow of SmartCut or Nano-Cleave. NanoCleave process does not require the last polishing step. . . . . . . . . 5 5

1.3 Cross-sections of two basic SOI waveguide structures: (a) a strip (or channel) waveguide and (b) a ridge (or "rib" in some literature) waveguide.

1.4 Mode definitions and electric field distributions in a strip waveguide. From [22]. 9

1.5 Effective index method (EIM): (a) a 3-D ridge waveguide is split into three parts: a core region and two cladding regions. Each region is treated as a 2-D slab waveguide to obtain $n_{\text {eff1 }}$ and $n_{\text {eff2 }}$. (b) The $n_{\text {eff1 }}$ and $n_{\text {eff2 }}$ are used to construct another three-layer slab waveguide to obtain the final effective index. . . . . . . . . . . . . . . . . . 10

1.6 Examples of element discretization in FEM waveguide analysis. The number of mesh elements is $N=2752 \ldots \ldots \ldots \ldots$

1.7 The cross-section of a ridge waveguide in an SOI platform: geometry parameter definitions. . . . . . . . . . . . . . . . . . . . 14

1.8 The single mode condition for SOI ridge waveguides: dotted curve (Eq. 1.6), solid curve (Eq. 1.6 with a modified -0.05 constant), and single and multimode experimental data points. From [21] . . . . . . . . . . 
1.9 The electric field profiles of the (a) TE-polarized and (b) the TM-polarized mode of a typical SOI ridge waveguide. The waveguide has a ridge width of $2.5 \mu \mathrm{m}$, an etch depth of $1.5 \mu \mathrm{m}$, and a ridge height of $2.2 \mu \mathrm{m}$. . . . 17

2.1 Cross-section of a silica-on-silicon planar waveguide. . . . . . . . .

2.2 Schematic layout of a polarization insensitive arrayed waveguide grating device fabricated using $\mathrm{SiO}_{2}-\mathrm{GeO}_{2} / \mathrm{SiO}_{2}$ on a $\mathrm{Si}$ substrate. A halfwave plate is inserted in the center of the grating section. From [41]. . . . . .

2.3 Schematic of the arrayed waveguide multiplexer. The shaded patch is the amorphous Si film (a-Si) for stress compensation. From [43] . . . . . .

2.4 Schematic of an arrayed waveguide grating device with stress relief grooves for birefringence compensation. From [45]. The $\mathrm{SiO}_{2}$-on-Si waveguide cross-section is also shown in the inset diagram. The groove had an etch depth of $d_{\text {etch }}=5.8 \mu \mathrm{m}$ and a width of $75 \mu \mathrm{m}$, the gap increment between two adjacent arrayed waveguides was $36 \mu \mathrm{m} . \ldots \ldots \ldots$

2.5 Schematic layout of a 48-channel diffraction grating based waveguide demultiplexer with a polarization compensator. Inset: an SEM image of the etched diffraction grating. From $[33] \ldots \ldots \ldots \ldots$

2.6 Schematic layout of a planar waveguide balanced Mach-Zehnder interferometer. The device length is $25 \mathrm{~mm}$ with a $3.8 \mathrm{~mm}$-long Bragg grating in each arm. From $[51] \ldots \ldots \ldots \ldots \ldots$

2.7 Birefringence as a function of waveguide width. The guiding structure was InGaAsP. Experimental results (data points) were compared with simulation results to determine the width of the strip waveguide. From [53]. . .

2.8 Schematic diagram of a polarization compensated waveguide grating router on InP. The InP cladding in the triangular patch section was $0.2 \mu \mathrm{m}$ thick while the cladding thickness was $1 \mu \mathrm{m}$ everywhere else. From [57] . . . . 
2.9 Polarization independent SOI waveguide bends: (a) schematic diagram of a waveguide $90^{\circ}$-bend structure with a radius of $4 \mu \mathrm{m}$ and the singlemode strip waveguide has a cross-section dimension of $0.35 \times 0.35 \mu \mathrm{m}^{2}$ (adapted from [59]); and (b) SEM image of a fabricated U-shaped (180 $)$ bend with a radius of $2.75 \mu \mathrm{m}$ and the strip waveguide has a cross-section of $0.32 \times 0.45 \mu \mathrm{m}^{2}$ (adapted from $\left.[60]\right) \ldots \ldots \ldots \ldots$

2.10 SEM image of the smallest reported AWG demultiplxer in SOI. The wire waveguides have a cross-section dimension of $0.32 \times 0.45 \mu \mathrm{m}^{2}$ and the overall device size is $\sim 70 \times 60 \mu \mathrm{m}^{2}$. From [61]. . . . . . . . .

2.11 Schematic configuration of a polarization insensitive MMI coupler using SOI. The MMI has the following dimensions: $W_{\mathrm{MMI}}=3.86 \mu \mathrm{m}, L_{\mathrm{MMI}}=$ $15.3 \mu \mathrm{m}$. The wire waveguides have square cross-sections with $W_{\mathrm{co}}=$ $H_{\mathrm{co}}=0.4 \mu \mathrm{m}$. From $[63] \ldots \ldots \ldots \ldots \ldots \ldots$

2.12 Waveguide geometries that satisfy both single-mode and birefringencefree condition simultaneous for various waveguide ridge heights: (a) at $\lambda_{0}=1530 \mathrm{~nm}$ (From Ref. [30]), and (b) at $\lambda_{0}=1550 \mathrm{~nm}$ (From Ref. [27]).

2.13 (a) Microscope image of an SOI AWG demultiplexer with two identical polarization compensators in the slab FPR regions (adapted from $[7,66]$ ); (b) cross-section of the polarization compensation region: an etched compensator for the AWG device in Ref. [7] (left) and a silicon-oxide-silicon (SOS) compensator for the device in Ref. [66] (right). . . . . . . . . . .

2.14 Microscope image of a compact polarization independent racetrack ring resonator on SOI. The cross-section of the directional coupler is shown in the inset. From $[67] . \ldots \ldots \ldots \ldots \ldots$ 
2.15 A schematic plan view of an SOI AWG device. The ridge waveguides has the following dimensions: width $W=5.8 \mu \mathrm{m}, H=4.3 \mu \mathrm{m}, D=1.7 \mu \mathrm{m}$. The truncated triangular region 30 is the patterned thermal oxide patch. From $[68] \ldots \ldots \ldots \ldots \ldots \ldots \ldots$

2.16 (a) Cross-section of the SOI ridge waveguide: $W=H=1.5 \mu \mathrm{m}, H-h=$ $0.9 \mu \mathrm{m}$; (b) a ring resonator using an MMI coupler with a polarization independent MMI coupler: $R=200 \mu \mathrm{m}$; (c) a close-up of the MMI coupler: $W_{\mathrm{MMI}}=6 \mu \mathrm{m}$ and $L_{\mathrm{MMI}}=54 \mu \mathrm{m}$. From $[70,71] \ldots \ldots . \ldots 40$

2.17 Polarization splitter geometry with waveguide phased array configuration based on an $\mathrm{SiO}_{2} / \mathrm{Al}_{2} \mathrm{O}_{3} / \mathrm{SiO}_{2}$ waveguide structure on a silicon substrate. From $[73] \ldots \ldots \ldots \ldots \ldots \ldots \ldots$

2.18 (a) Configuration of a MZI-based polarization splitter using a stressed a-Si film; (b) the cross-section geometry of the upper MZI arm: the core had a dimension of $8 \times 8 \mu \mathrm{m}^{2}$, and the a-Si film was $7 \mu \mathrm{m}$ thick and had a width ranged from 50 to $100 \mu \mathrm{m}$. From $[42] \ldots \ldots \ldots \ldots$

2.19 Schematic diagrams of an electro-optic polarization splitter with an MZI configuration. Waveguides were fabricated using InGaAsP-InP. From [75].

2.20 Schematic diagram of a $1 \times 2$ asymmetric Y-branch polarization splitter. From [76]

2.21 Schematic of an MZI-based polarization splitter, with two MMI $3 \mathrm{~dB}$ couplers. The shaded area indicates the $\mathrm{Ti}$ metal-SiO $\mathrm{S}_{2}$ cladding on the top of the InP waveguide. From [79] . . . . . . . . . . . . . . . . . 46

2.22 Schematic of a directional coupler based polarization splitter. (a) Top view of the device layout. At a chosen coupling length, $L_{c}$, the mode profiles show that (b) the TE mode stays in bar state; (c) the TM mode transfers to the cross-state. From $[81] \ldots \ldots \ldots \ldots \ldots$ 
2.23 (a) Cross-section of the SOI ridge waveguide; (b) schematic layout of the MZI based polarization splitter. From [12] . . . . . . . . . . . . .

3.1 The influence of core dimensions on waveguide geometrical birefringence $\left(\Delta n_{\text {geo }}\right)$ for a geometry shown in the inset. . . . . . . . . . 5

3.2 Typical simulation calculation window: a $100 \times 100 \mu \mathrm{m}^{2}$ window for stress analysis and a $8 \times 5 \mu \mathrm{m}^{2}$ window for modal analysis. Inset: a close-up view of a typical ridge waveguide in the mode calculation window. . . . .

3.3 The $x$ and $y$-components of the stress distributions in an SOI ridge waveguide: (a) the stress field $\sigma_{x}$ and (b) the stress field $\sigma_{y}$. The buried oxide layer thickness is $0.37 \mu \mathrm{m}$, the upper oxide cladding thickness is $1 \mu \mathrm{m}$, and Si ridge height and width are $2.2 \mu \mathrm{m}$ and $1.8 \mu \mathrm{m}$, respectively. . . . .

3.4 Simulation window size. The cladding film thickness is $t$, the substrate thickness is $d$. Usually $d \gg t$. The parameter $L_{\min }$ defines the minimum distance between the edge of the ridge waveguide and the calculation window boundary to avoid edge effects. . . . . . . . . . . . . . .

4.1 An SEM image of the cross section of a ridge waveguide in the arrayed grating section of a test AWG device (see Fig. 5.1) . . . . . . . . . .

4.2 Influence of the upper oxide cladding thickness $(t)$ on the effective index $\left(n_{\mathrm{eff}}^{\mathrm{TM}}\right.$ and $\left.n_{\mathrm{eff}}^{\mathrm{TE}}\right)$ and the geometrical birefringence $\left(\Delta n_{\mathrm{geo}}=n_{\mathrm{eff}}^{\mathrm{TM}}-n_{\mathrm{eff}}^{\mathrm{TE}}\right)$. The considered trapezoidal ridge waveguide has the following geometry parameters: $W_{1}=1.5 \mu \mathrm{m}$, sidewall slope $\theta=54^{\circ}, D=1.5 \mu \mathrm{m}$, and $H=2.2 \mu \mathrm{m}$. No stress is considered here. . . . . . . . . . 
4.3 Fundamental TE mode shapes with equal electric field contours $(0.5,0.1$, and 0.01 of the maximum fields) in SOI ridge waveguide. The ridge height is $2.2 \mu \mathrm{m}$, the etch depth is $1.5 \mu \mathrm{m}$. (a) A rectangular ridge with a width of $2 \mu \mathrm{m}$; and (b) a trapezoidal ridge with a top width $W_{1}$ of $2 \mu \mathrm{m}$ and a sidewall angle of $54^{\circ} \ldots \ldots \ldots \ldots \ldots$. . . . . . . . . . . . 74

4.4 The variation of waveguide geometrical birefringence $\left(\Delta n_{\text {geo }}\right)$ with sidewall slope $(\theta)$ for 3 different etch depths $(D)$. The Si ridge has an upper width $\left(W_{1}\right)$ of $1.5 \mu \mathrm{m}$ and the total height of the core is $2.2 \mu \mathrm{m} . \ldots \ldots$. . . .

4.5 Etch depth influence on waveguide geometrical birefringence $\left(\Delta n_{\text {geo }}\right)$ for rectangular and trapezoidal waveguides with sidewall slope of $54^{\circ}, 72^{\circ}$, $87^{\circ}$ and $90^{\circ}$, in the absence of stress. The Si ridge has a height $H$ of 2.2 $\mu \mathrm{m}$ and a top ridge width $W_{1}$ of $1.5 \mu \mathrm{m}$ (bottom ridge width $W_{2}$ varies according to slope $\theta) \ldots \ldots \ldots \ldots \ldots \ldots$

4.6 Ridge width influence on waveguide effective index and geometrical birefringence for trapezoidal and rectangular waveguides. Shown are (a) effective mode indices of TE and TM polarizations in trapezoidal $\left(\theta=54^{\circ}\right)$ and rectangular $\left(\theta=90^{\circ}\right)$ ridge waveguides and (b) geometrical birefringence $\left(\Delta n_{\text {geo }}\right)$. The Si ridge etch depth is fixed at $1.5 \mu \mathrm{m}$, and ridge height $H$ is

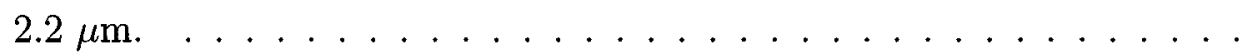

4.7 Electric field distribution of the fundamental TE mode for (a) a rectangular waveguide and (b) a trapezoidal waveguide with a sidewall angle of $54^{\circ}$. Both waveguides have $W_{1}=0.1 \mu \mathrm{m}, D=1.5 \mu \mathrm{m}, H=2.2 \mu \mathrm{m} . \quad \ldots \ldots \quad 78$

4.8 Geometrical birefringence $\Delta n_{\text {geo }}$ as a function of the parameter $r=1-$ $D / H$ and the cross-section aspect ratio $W / H$ in rectangular ridge waveguides. Here the silicon core thickness $H$ is fixed at $2 \mu \mathrm{m} . \ldots . . . . .79$

xvii 
4.9 Geometrical birefringence $\Delta n_{\text {geo }}$ as a function of the ridge etch ratio $r$, for different ridge height $H$ as indicated in the figure. The waveguide geometry is shown in the inset, with $W / H=0.8$. . . . . . . . . . . 80

4.10 Stress distributions along $x$-direction $\left(\sigma_{x}\right)$ for (a) a rectangular waveguide and (b) a trapezoidal waveguide with a sidewall angle of $54^{\circ}$. Both waveguides have $W_{1}=1.5 \mu \mathrm{m}, D=1.5 \mu \mathrm{m}$, and $H=2.2 \mu \mathrm{m}$. The oxide cladding thickness is $t=0.7 \mu \mathrm{m} . \ldots \ldots \ldots$

4.11 Influence of the upper cladding with different levels of stress on the effective indices of the TE and TM modes. The trapezoidal waveguide geometry is identical to that defined in Fig. 4.2: $\theta=54^{\circ}, W_{1}=1.5 \mu \mathrm{m}, D=1.5 \mu \mathrm{m}$, and $H=2.2 \mu \mathrm{m} . \ldots \ldots \ldots \ldots \ldots \ldots \ldots \ldots \ldots \ldots \ldots \ldots \ldots \ldots \ldots \ldots \ldots$

4.12 Influence of upper cladding under different levels of stress on waveguide birefringence. The bottom curve corresponds to the geometrical birefringence (no stress is included in the calculations of waveguide effective indices). The waveguide geometry is identical to that defined in Fig. 4.2

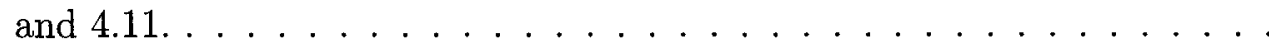

4.13 Temperature sensitivity of (a) stress in the upper cladding film $\sigma_{\text {film }}$ (cooling from 400 to $20^{\circ} \mathrm{C}$ ); (b) the stress-induced effective index change and stress-induced birefringence $\Delta n_{\text {stress }}$. The operating temperature is assumed to vary from $10^{\circ} \mathrm{C}$ to $80^{\circ} \mathrm{C}$. The waveguide has the following specifications: $W=1.5 \mu \mathrm{m}, D=1.4 \mu \mathrm{m}, H=2.2 \mu \mathrm{m}, t=1 \mu \mathrm{m}$, and the

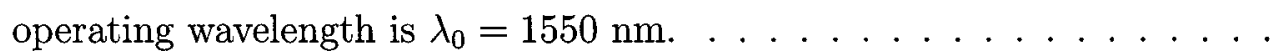

4.14 The wavelength dependency on the effective index of the TE and TM (i) with no stress ( $n_{\mathrm{eff}}^{\mathrm{TE}}$ and $n_{\mathrm{eff}}^{\mathrm{TM}}$ ); (ii) with $\sigma_{\text {film }}=-330 \mathrm{MPa}$. The waveguide has the following dimensions: $W=1.5 \mu \mathrm{m}, D=1.4 \mu \mathrm{m}, H=2.2 \mu \mathrm{m}$, and $t=1 \mu \mathrm{m} \ldots \ldots \ldots \ldots \ldots \ldots \ldots \ldots \ldots \ldots \ldots \ldots \ldots \ldots \ldots \ldots \ldots \ldots$

xviii 
4.15 Etch depth influence on stress-induced birefringence $\left(\Delta n_{\text {stress }}\right)$ for a rectangular $\left(\theta=54^{\circ}\right)$ and a trapezoidal $\left(\theta=90^{\circ}\right)$ ridge waveguide. The $0.7 \mu \mathrm{m}$ upper oxide cladding film is under a compressive stress of $-300 \mathrm{MPa}$. The waveguide geometry is identical to that defined in Fig. 4.5: $W_{1}=1.5 \mu \mathrm{m}$ and $H=2.2 \mu \mathrm{m} \ldots \ldots \ldots \ldots \ldots \ldots \ldots \ldots \ldots \ldots \ldots \ldots \ldots \ldots \ldots$

4.16 Ridge width influence on waveguide effective index and birefringence for trapezoidal and rectangular waveguides. Shown are (a) effective mode indices of TE polarization with stress (solid curves) and without stress (dashed curves) in trapezoidal $\left(\theta=54^{\circ}\right)$ and rectangular $\left(\theta=90^{\circ}\right)$ ridge waveguides and (b) geometrical birefringence (dashed curves) and total birefringence $\left(\Delta n_{\text {stress }}+\Delta n_{\text {geo }}\right)$ under a compressive stress of $\sigma_{\text {film }}=-300$ MPa (solid curves). The oxide cladding thickness is $t=0.7 \mu \mathrm{m}$. Identical waveguide geometries as in Fig. 4.6 are used: $D=1.5 \mu \mathrm{m}, H=2.2 \mu \mathrm{m}$. .

4.17 Stress-induced birefringence $\Delta n_{\text {stress }}$ as a function of the etch ratio $r=$ $1-D / H$ and the cross-section aspect ratio $W / H$ in rectangular ridge waveguides. Here the silicon core thickness $H$ is fixed at $2 \mu \mathrm{m}$. The oxide cladding is assumed to be $1.5 \mu \mathrm{m}$ thick and a compressive stress of -100 $\mathrm{MPa}$ is assumed.

4.18 Stress-induced birefringence $\Delta n_{\text {stress }}$ (waveguide geometry shown inset) as a function of the the oxide thickness. The stress level in the oxide cladding is assumed to be $\sigma_{\text {film }}=-100 \mathrm{MPa} \ldots \ldots \ldots \ldots$

4.19 The variation of modal birefringence with waveguide width for a photonic wire with dimensions shown in the inset.

5.1 A top view of a fabricated SOI AWG demultiplexer. . . . . . . . . 
5.2 SEM images of the cross section of a ridge waveguide in the arrayed grating section (see Fig. 5.1). Shown are (a) a dry-etched waveguide with a PECVD deposited oxide cladding (same as Fig. 4.1) and (b) a wet-etched waveguide without cladding deposition. . . . . . . . . . . . . .

5.3 A schematic of a bent wafer for stress calculation. . . . . . . . . . . . . 101

5.4 A schematic of the experimental setup for waveguide testing. . . . . . . 102

5.5 Measured output TE (dashed) and TM (solid) spectra of a wet etched 200 $\mathrm{GHz}$ 9-channel SOI AWG with ridge height $H=2.2 \mu \mathrm{m}$, ridge widths $W_{1}=1.1 \mu \mathrm{m}$ and $W_{2}=3.8 \mu \mathrm{m}$, and etch depth $D=1.47 \mu \mathrm{m}$. There is no upper oxide cladding. . . . . . . . . . . . . . . . .

5.6 The measured (data points) and calculated (solid curves) wavelength shift $(\Delta \lambda)$ in the demultiplexer's spectra for varying oxide thickness. Shown are (a) dry-etched waveguides and (b) wet-etched waveguides, with stress of $\sigma_{\text {film }}=-320 \mathrm{MPa}$. The ridge geometrical parameters are shown in the inset of (b). The ridge edge depth was measured as $1.47 \mu \mathrm{m}$. Stress was determined from the measurements on wafer bow radius. . . . . . . . . 105

5.7 The measured output TE (dashed) and TM (solid) spectra of a wet etched $200 \mathrm{GHz}$ 9-channel SOI AWG after stress compensation. The geometry of the ridge waveguides is identical to that defined in Fig. 5.5. An upper oxide cladding thickness of $0.6 \mu \mathrm{m}$ was used to compensate and eliminate the large polarization dependent wavelength shift.

6.1 Schematic diagram of a basic balanced MZI-based polarization splitter with two $3 \mathrm{~dB}$ couplers. The oxide cladding patch provides the necessary phase shift for polarization splitting. . . . . . . . . . . . . . . 110

6.2 Schematic diagram of an MZI-based polarization filter with two Y-junctions as the input and output combiners. . . . . . . . . . . . . 111 
6.3 Schematic diagram of an MZI-based polarization splitter with one Yjunction input coupler and one $3 \mathrm{~dB}$ directional coupler as the output combiner.

6.4 Schematic diagram of an MZI-based polarization splitter with a Y-junction input coupler and an MMI output coupler. . . . . . . . . . . . . .

6.5 A $2 \times 2$ MMI coupler: (a) the schematic layout; (b) the light intensity patterns along the longitudinal and transversal directions, producing twofold images $[70] \ldots \ldots \ldots \ldots \ldots \ldots \ldots$

6.6 Schematic diagram of an MZI-based polarization splitter with two 3dB directional couplers as both the input and the output combiners. . . . . . 118

6.7 Schematic diagram of an MZI-based polarization splitter with MMI couplers as both the input and the output combiners. . . . . . . . . .

6.8 Summary of ridge cross-section dimensions for the ridge waveguides used in our polarization splitter devices. . . . . . . . . . . . .

6.9 Triangular patch to cover the split region for $\mathrm{Y}$-junction fabrication. . . .

6.10 Polarization and wavelength insensitive performance of a $3 \mathrm{~dB}$ MMI device. The example MMI has the following dimensions: $L_{\mathrm{MMI}}=186 \mu \mathrm{m}$ and $W_{\mathrm{MMI}}=10.5 \mu \mathrm{m} \ldots \ldots \ldots \ldots \ldots \ldots$

6.11 An MZI-based polarization splitter with symmetric $3 \mathrm{~dB}$ couplers: shown are the simulated TE and TM power in the polarization splitter output ports [(a) top port and (b) bottom port] as a function of the upper oxide cladding patch length. The stress is $\sigma_{\text {film }}=-330 \mathrm{MPa}$ and the cladding thickness $t$ is $1 \mu \mathrm{m}$. The ridge waveguides have the following dimensions: $H=2.2 \mu \mathrm{m}, W=1.5 \mu \mathrm{m}$, and $D=1.4 \mu \mathrm{m} \ldots \ldots \ldots \ldots$ 
6.12 Output power of the TE and TM modes as a function of operating wavelength in an MZI-based polarization splitter with symmetric $3 \mathrm{~dB}$ couplers. The same device parameters defined in Fig. 6.11 are used here. The oxide

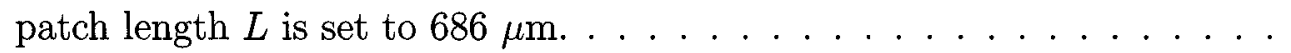

6.13 Optical masks of Mach-Zehnder interferometer based polarization test devices. The mask layout also includes ring resonator devices which are not discussed in this thesis. The typical MZI devices are roughly $2.5 \mathrm{~mm} \times$

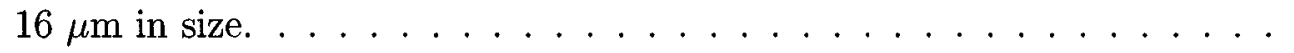

6.14 (SEM images of fabricated dry-etched ridge waveguides: (a) cross-section of a ridge waveguide; (b) sidewall roughness along an etched ridge. . . . .

6.15 Microscope plan view of the fabricated MZI-based polarization filters/splitters: (a) a Y-junction, (b) a directional coupler, (c) an MMI coupler, (d) a deposited oxide patch on the bottom MZI arm. . . . . . . . . . . .

6.16 Close-up image of (a) the split region of a $\mathrm{Y}$-junction and (b) the ridge waveguides/slab junction of an MMI coupler. . . . . . . . . . . . .

6.17 An SEM image of the oxide cladding on one MZI arm. . . . . . . . .

6.18 A picture of the testing stage for the waveguide polarization splitter measurements. A tapered single-mode fiber is used to collect the device output

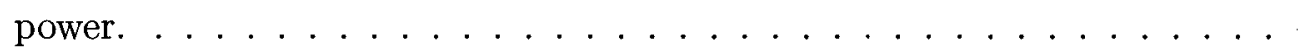

6.19 Broadband MZI-based polarization splitters: (a) MZI with asymmetric 3 $\mathrm{dB}$ couplers (i.e., one Y-junction + one MMI); (b) MZI with two symmetric MMI couplers. The ridge waveguides for both devices have the following dimensions: $W=1.5 \mu \mathrm{m}, D=1.4 \mu \mathrm{m}$, oxide cladding is under a compressive film stress $\sigma_{\text {film }}=-345 \mathrm{MPa}$, with a length $L=800 \mu \mathrm{m}$ and a thickness $t=1.2 \mu \mathrm{m}$. The MMIs have $W_{\mathrm{MMI}}=10.5 \mu \mathrm{m}$ and

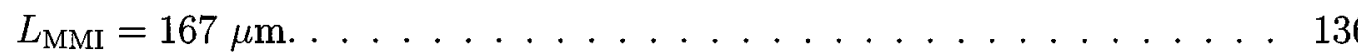


6.20 Simulated stress distribution for ridge waveguides. The oxide cladding has (a) full sidewall coverage and (b) has zero sidewall coverage. The dimensions of the ridge waveguide are: $W=1.5 \mu \mathrm{m}, H=2.2 \mu \mathrm{m}, D=$ $1.4 \mu \mathrm{m}$ and $t=1 \mu \mathrm{m} \ldots \ldots \ldots \ldots \ldots \ldots$

7.1 (a) Layout of a typical arrayed waveguide grating (AWG) structure; (b) Geometry of the receiver free-propagation region. From [94]. . . . . . . . 141

7.2 Geometrical layout of a waveguide array based thermo-optical switch, with the optical waveguides, chromium heaters, and aluminum leads. From [95]. 143

7.3 A schematic diagram of a zero-order arrayed waveguide grating. The waveguides in the arrayed section have identical channel length. The green enclosed area indicates the size of the selectively deposited oxide cladding

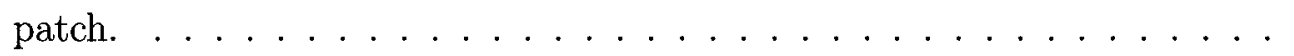

7.4 Calculated bending loss vs. radius of curvature for ridge waveguides with $W=D=1.5 \mu \mathrm{m}$ and $H=2.2 \mu \mathrm{m}$ : (a) fundamental and first-order TE modes and (b) fundamental and first-order TM modes. . . . . . . . . . . 146

7.5 The geometry of the output side free-propagation region (FPR) of a zeroorder AWG device. The output aperture and the image plane follow a typical design of a Rowland mounting. . . . . . . . . . . . . . 147

7.6 The spatial separation of the output TE and TM polarizations as a function of operation wavelength. The AWG has an oxide patch length increment $\Delta L=16.2 \mu \mathrm{m}$. The oxide cladding has a thickness of $0.8 \mu \mathrm{m}$ with a stress of $\sigma_{\text {film }}=-300 \mathrm{MPa}$. The ridge waveguides have the following

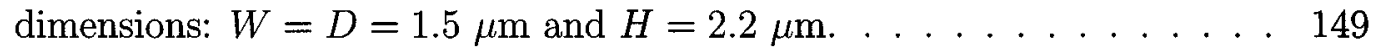

7.7 The geometry of the input free-propagation region (FPR) of a zero-order AWG device. . . . . . . . . . . . . . . . . . 150

7.8 Optical masks of zero-order arrayed waveguide grating (AWG) test devices. The overall device size of an AWG-based splitter is $\sim 12 \mathrm{~mm} \times 4 \mathrm{~mm}$. . . 153

xxiii 
7.9 SEM images of (a) a dry-etched ridge waveguide and (b) a wet-etched waveguide

7.10 SEM images of the wet-etched oxide cladding on (a) the dry-etched ridge waveguides and (b) the wet-etched ridge waveguides.

7.11 A top view of our fabricated wavelength independent zero-order AWG polarization splitter. There are 100 waveguides in each arrayed section. The triangular windows define the oxide cladding patch regions. . . . . .

7.12 Measured wavelength dependence of a zero-order AWG before oxide cladding deposition: (a) a dry-etched AWG and (b) a wet-etched AWG. The power of both polarizations were measured from the same output channel. All ridge waveguides in the AWGs are designed to have a width $W=2 \mu \mathrm{m}$ and an etch depth $D=1.5 \mu \mathrm{m} . \ldots \ldots \ldots \ldots$

7.13 Screen shots of the outputs of a functioning polarization splitter: (a) the TE polarization appears in channel II and (b) the TM polarization appears in channel I. . . . . . . . . . . . . . . . . . . . .

7.14 Measured wavelength dependence of zero-order AWG-based polarization splitter with an oxide cladding patch: (a) a dry-etched AWG with an oxide patch length increment $L=20.1 \mu \mathrm{m}$ and (b) a wet-etched AWG with $L=23.3 \mu \mathrm{m}$. The ridge waveguides have a width and etch depth of $2 \mu \mathrm{m}$ and $1.5 \mu \mathrm{m}$, respectively. The oxide cladding has a thickness of 1 $\mu \mathrm{m}$ and a stress level of $\sigma_{\text {film }}=-340 \mathrm{MPa} \ldots \ldots \ldots$

7.15 A wet-etched zero-order AWG-based polarization splitter with an extinction ratio as high as $-20 \mathrm{~dB}$. The ridge waveguides have a width and etch depth of $W=D=1.5 \mu \mathrm{m}$. The oxide cladding is under a stress level of $\sigma_{\text {film }}=-340 \mathrm{MPa}$, with a thickness of $1 \mu \mathrm{m}$ and a length increment $L=16.4 \mu \mathrm{m} . \ldots \ldots \ldots \ldots \ldots \ldots \ldots$ 
B.1 Algorithm for drawing the layout of a zero-order AWG device (left half) with equal spacing at the center of the arrayed section. The constant spacing between adjacent waveguides at the middle of the arrayed section

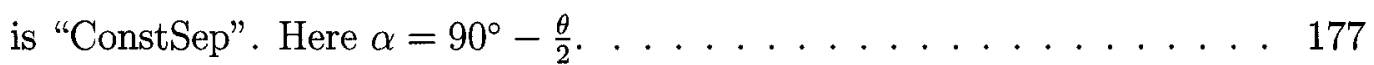

$\mathrm{xxy}$ 


\section{Chapter 1}

\section{Introduction}

Integrated circuits are ubiquitous and have turned into an inextricable part of our modern society. Ever since the first silicon transistor demonstration in the 1950s, silicon has been the dominant platform for the integrated microelectronics circuits, and it will continue to be the material of choice in the foreseeable future [1]. Silicon based integrated circuits can be found in computers, cars, cellular phones, and other essential appliances that affect our everyday life. The continuing demand for increased functionality, enhanced performance, improved reliability, and reduced cost remains the strongest driving force towards further downscaling of the device feature size. In recent years, monolithic integration of electronic and optic circuits on a single substrate for improved performance and reduced cost has become an active research topic around the world. Amongst the available material systems, the high-index contrast "silicon-on-insulator (SOI)" platform has received tremendous interest due to its potential for miniaturization of monolithic photonic integrated circuits [2].

\subsection{Silicon-on-insulator (SOI)}

SOI is known for its compatibility with the current CMOS technologies for making compact, highly integrated, and multifunctional photonic devices. The SOI platform not only 
uses silicon as the substrate but also as the waveguiding core material. The SOI material system has a number of distinct advantages over the other commonly used platforms such as the III-V semiconductor compounds and polymers. First, the high refractive index contrast between the silica cladding and the silicon waveguide core facilitates the confinement and guiding of light in structures within submicron dimensions. In addition, the mature silicon microfabrication technology establishes a firm foundation for making low-cost and compact integrated photonic devices. Furthermore, SOI technology offers tremendous potential for monolithic integration of electronic and photonic devices on the same substrate. A wide range of optical devices has been realized on the SOI platform, including optical modulators [3-5], optical switches [6], optical spectrometers [7], variable optical attenuators [8], optical add/drop filters [9,10], polarization-mode converters [11], polarization splitters [12], and even light sources [13].

Although the high index contrast in the SOI system allows significant reduction in component foot-print, challenges such as the control of polarization still remain the biggest obstacles to the development of viable microphotonic commercial products. Polarization is important in a wide range of applications, from common household items such as sunglasses, to advanced applications such as receivers in coherent optical communication systems, wavelength demultiplexing and high resolution spectroscopy in telecommunication applications, polarization microscopes and sensors for biomedicine applications, radar displays in defense and security systems, saccharimeters for measuring sugar content in chemistry, and polariscopes for determining strain patterns in mechanical engineering [14]. Polarization analysis, control, and manipulation have become increasingly critical in the design and the operation of optical devices. This thesis explores polarization control for SOI integrated optical devices and proposes feasible and practical applications.

The configuration of an SOI wafer is shown in Fig. 1.1. It consists of a core layer of single-crystal silicon, a thin buried $\mathrm{SiO}_{2}$ layer which acts as the bottom cladding, and 


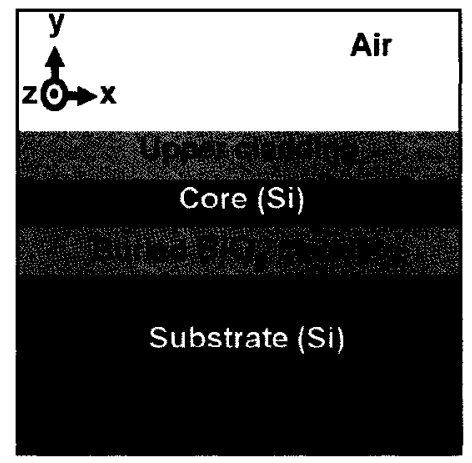

Figure 1.1: Cross-section of an SOI wafer: a slab waveguide.

a silicon substrate. The buried $\mathrm{SiO}_{2}$ cladding layer has a material refractive index of $\left(n_{\mathrm{SiO}_{2}} \approx 1.5\right)$, which is much lower than that of the silicon core layer $\left(n_{\mathrm{Si}} \approx 3.5\right)$, at the operating wavelength of $1550 \mathrm{~nm}$. As a result, the silicon core layer and the buried oxide layer can be used to form an optical waveguide. Typically, an upper cladding layer is applied over the silicon core in planar waveguides to reduce surface contamination or to provide electrical isolation [15]. An $\mathrm{SiO}_{2}$ film is often chosen as the upper cladding, although nitride or polymers are also used. In microelectronics and micromechanics, the optimal thickness and the physical property of the silicon core layer, as well as the thickness of the buried $\mathrm{SiO}_{2}$ cladding layer, are application specific. In silicon microphotonics, the buried oxide provides optical isolation of the silicon core from the substrate. The exact thickness of the buried $\mathrm{SiO}_{2}$ cladding does not have a significant effect on the waveguide, as long as it is thick enough to prevent optical field penetration into the substrate. However, the choice of the material and the properties of the upper cladding does affect the overall performance of the waveguide. Thus the term "cladding" in this thesis refers to the upper cladding, unless otherwise specified.

\subsubsection{Fabrication of SOI Wafers}

This section describes the five basic approaches to the fabrication of commercial SOI wafers: separation by implanted oxygen (SIMOX), bond and etch-back (BESOI), SmartCut, NanoCleave, and epitaxial layer transfer (ELTRAN). 
One of common methods for mass production of SOI wafers is the Separation by IMplanted OXygen (SIMOX) process [16]. It employs a high-dose ion implantation of oxygen into plain silicon wafers. In a SIMOX process, a total implantation dose of $10^{18} \mathrm{~cm}^{-2}$ with an energy of up to $200 \mathrm{keV}$ is required to form a buried $\mathrm{SiO}_{2}$ layer $500 \mathrm{~nm}$ below the wafer surface [17]. A final high temperature annealing step at approximately $1300^{\circ} \mathrm{C}$ [2] is usually performed to allow the implanted oxygen atoms to migrate and combine with silicon atoms to form a buried $\mathrm{SiO}_{2}$ layer of uniform thickness, which ensures that the silicon overlayer is free of implantation induced defects. The buried $\mathrm{SiO}_{2}$ layer and the silicon core cannot usually be made greater than $500 \mathrm{~nm}$ in thickness. The implant energy and dose determine the final thickness and depth of the buried $\mathrm{SiO}_{2}$ layer. Besides the limited achievable core thickness, the high wafer cost and long processing time are the main disadvantages of the SIMOX approach for most optical applications.

Alternatively, SOI wafers can be fabricated by the Bond and Etch-back method (BESOI). This process involves the bonding of two oxidized silicon wafers (one is a devicequality "donor wafer" and the another is a "handle wafer"), forming a sandwiched oxide layer in between the two silicon wafers. The two wafers are initially brought into contact at room temperature, followed by a thermal annealing at temperatures as high as $1100^{\circ} \mathrm{C}[2,17]$. The bonded pair then undergoes a wafer thinning step by etching and chemical mechanical polishing (CMP) that leaves a thin, device-quality layer of single crystal silicon overlayer on top of the oxide layer (the buried oxide) of the "handle wafer". The silicon overlayer thickness of the BESOI wafers is limited to a minimum of around $5 \mu \mathrm{m}[2,17]$ due to technical challenges in removing excess silicon during the last wafer thinning process. Besides the concern of the surface uniformity of the overlayer surface, wafer waste is another problem of this approach.

The layer-transfer approach has led to the development of the other three production methods for fabrication of SOI wafers: the SmartCut, NanoCleave, and ELTRAN. The 
former two processes adopt steps from the SIMOX and BESOI methods. They both employ a high-dose ion implantation (using hydrogen, for example), to form a weakened silicon layer in the thermally oxidized "donor wafer". The implantation dosage of the hydrogen ion is usually on the order of $10^{17} \mathrm{~cm}^{-2}$, and the distance from the wafer surface to the peak of the implantation profile is usually between $5 \mathrm{~nm}$ and $1.5 \mu \mathrm{m}[2,17-19]$. After bonding with a "handle wafer" as in a BESOI process, a special cleaving technique is used to split the donor wafer along the weakened ion penetration region. The SmartCut technique [18] involves a two-phase thermal treatment to split the wafer. Post-cleaving polishing is required to remove micro-roughness on the overlayer surface. In contrast, the NanoCleave [19] uses a gas pressured controlled cleaving process, and no post-cleaving CMP is needed. The process flow can be illustrated in Fig. 1.2. The ELTRAN (Epitaxial Layer TRANsfer) method has a similar process flow as presented in Fig. 1.2, except it does not use ion implantation to form the weakened splitting layer. Instead, it employs a

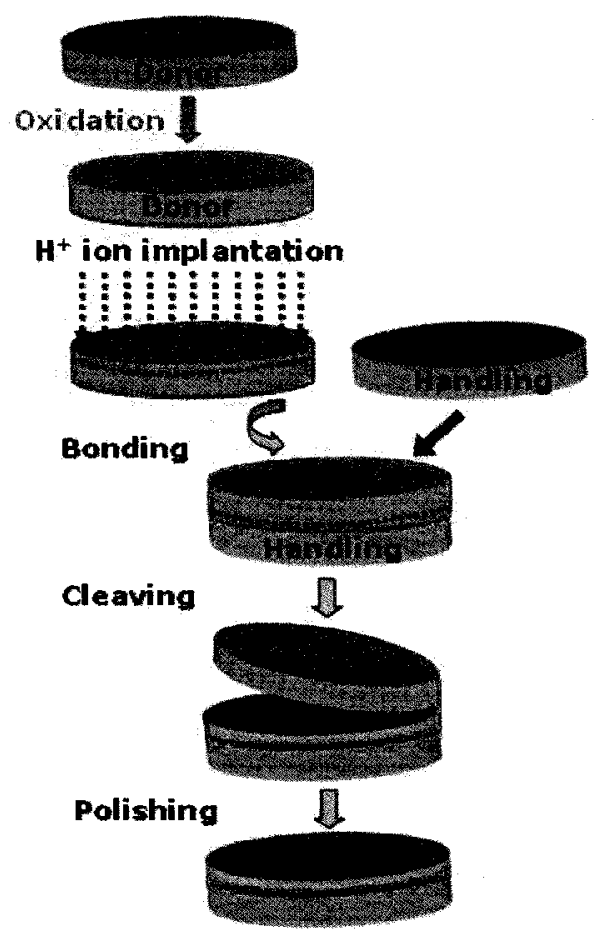

Figure 1.2: Schematic of fabrication process flow of SmartCut or NanoCleave. NanoCleave process does not require the last polishing step. 
layer of porous silicon below the surface of the "donor wafer", which is formed by anodic etching and annealing [17]. The SmartCut, NanoCleave, and ELTRAN methods share one significant efficiency: the split "donor wafer" can be recycled for future use.

In all the above mentioned fabrication method, the silicon overlayer thickness in general cannot be made larger than $1.5-2 \mu \mathrm{m}$. To achieve a thicker overlayer, it is possible to use the silicon overlayer as a seed layer for subsequent epitaxial growth [2]. The term "epitaxial growth" means that the silicon atoms in the overlayer are deposited with a matching crystalline structure of the seed layer. The most popular epitaxial silicon growth technique is chemical vapor deposition (CVD), where a gas mixture such as $\mathrm{SiHCl}_{3}$ and $\mathrm{SiH}_{2} \mathrm{Cl}_{2}$ are decomposed at the wafer surface. A high temperature, typically $>1000^{\circ} \mathrm{C}[2]$, is required for the chemical reaction.

Amongst the mentioned fabrication techniques of SOI wafers, the SmartCut technique has gained more popularity due to its flexibility, high quality and efficient use of silicon, with relatively low cost [18]. Although a high dose implantation is involved in forming the thin silicon overlayer, the small mass of the hydrogen ions leaves negligible damage after subsequent thermal processing. All the SOI wafers used in this project were of the SmartCut type, ordered from Soitec USA Inc. The purchased wafers had a silicon core thickness of $2.2 \mu \mathrm{m}$ that had been epitaxially grown from a $0.2 \mu \mathrm{m}$ seed silicon layer.

\subsubsection{SOI Waveguides}

With the low material refractive index of the upper and lower cladding layers (e.g, $n_{\mathrm{SiO}_{2}} \approx$ 1.5), light is guided and confined primarily in the high index silicon core layer (with $\left.n_{\mathrm{Si}} \approx 3.5\right)$. The index difference between the core and cladding $\left(\Delta n=n_{\mathrm{Si}}-n_{\mathrm{SiO}_{2}} \approx 2.0\right)$ is exceptionally high in SOI compared to waveguides based on glass or polymers $(\Delta n<$ 0.01 ), making it possible to tightly confine the mode.

An SOI wafer itself (Fig. 1.1) is a simple slab waveguide, offering a vertical confinement in the $y$-direction for light propagating along the $z$-direction in the silicon core 
layer. In general, waveguides with such a two-dimensional confinement are of limited practical use. A simple etching step can be used to make a three-dimensional waveguide. Fig. 1.3 shows two possible SOI waveguide structures, namely, the strip (or channel) and ridge (sometimes referred to as "rib") waveguides. Both types of waveguides experience an additional lateral confinement defined by the boundaries of the core in the $x$-direction. To achieve single mode operation (which will be discussed later in this chapter), a strip waveguide must be small in dimension (e.g., smaller than $350 \mathrm{~nm} \times 350 \mathrm{~nm}$ for a square cross-section), which can be challenging for coupling and fabrication. A single-mode ridge waveguide, on the other hand, can be larger in size, and hence is usually the preferred choice in device design. In reality, the ridge waveguide may never be truly single mode. However, if properly designed, it has been demonstrated theoretically and experimentally that the power carried by the higher-order modes will leak out of the waveguide over a very short traveling distance [20,21], leaving only the fundamental mode. Therefore, ridge waveguides with larger cross-sections and with much relaxed fabrication tolerances are still considered as quasi single-mode waveguides. Throughout this thesis SOI ridge waveguides are used for all theoretical analyses and experimental demonstrations.

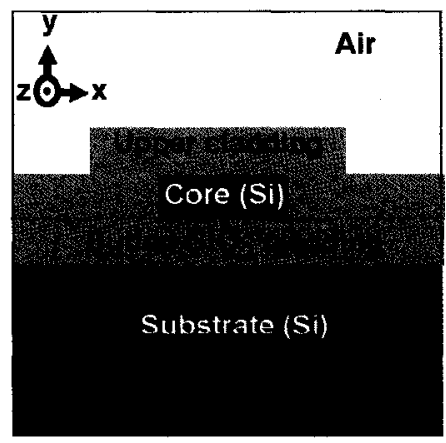

(a)

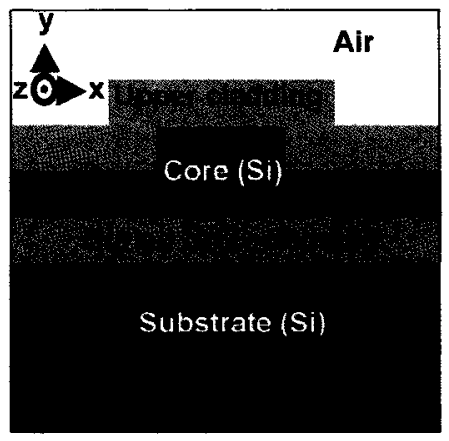

(b)

Figure 1.3: Cross-sections of two basic SOI waveguide structures: (a) a strip (or channel) waveguide and (b) a ridge (or "rib" in some literature) waveguide. 


\subsection{Waveguide Mode and Polarization}

This section first reviews the basic concepts of optical modes. Then the two commonly used modal analysis methods are described in detail. Finally, the waveguide polarization and its associated problems are discussed.

\subsubsection{Waveguide Modal Analysis}

Light propagation in waveguides is governed by the Maxwell equations. The optical modes of the waveguide are the eigen solutions to the Maxwell equations, subject to the appropriate boundary conditions imposed by the waveguide geometry. In the simplest slab waveguide system (e.g., Fig. 1.1), two orthogonally polarized modes can be supported: the transverse electric (TE) mode which is defined as the electric field perpendicular to the propagation direction and lying in the plane of the core layer, and the transverse magnetic (TM) mode which refers to the field distribution with the magnetic field perpendicular to the propagation direction and in the plane of the core layer (i.e., the electric field is normal to the core layer).

In a strip or a ridge waveguide shown in Fig. 1.3, light is confined two-dimensionally in both the $x$ - and $y$-direction and is guided along the propagation $z$-direction. The requirement for the field components defined for the slab waveguides, either $E_{y}=E_{z}=$ $H_{x}=0$ for $\mathrm{TE}$ or $H_{z}=H_{y}=E_{x}=0$ for $\mathrm{TM}$, is no longer valid because the propagating light must satisfy the boundary conditions imposed by the waveguide in both the $x$ - and $y$-direction. Even though pure TE and TM modes do not exist, we use the term "TE-like mode" to define the hybrid mode in which $E_{x}$ and $H_{y}$ components dominate, and "TMlike mode" for the mode in which $E_{y}$ and $H_{x}$ dominate. They are denoted as $E_{p q}^{x}$ and $E_{p q}^{y}$, respectively, where $p$ and $q$ are the number of the field extrema (maxima and minima) in the $x$ - and $y$-direction. Fig. 1.4 illustrates the electric field distributions of various modes in a strip waveguide. It should be pointed that the longitudinal component $E_{z}$ of 


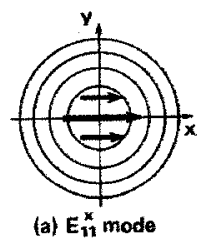

(a) $\mathbf{E}_{11}^{x}$ mode

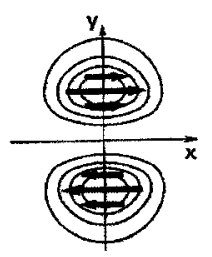

(c) $E_{12}^{x}$ mode

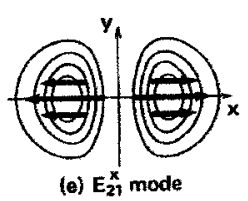

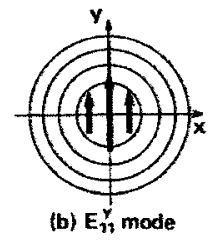

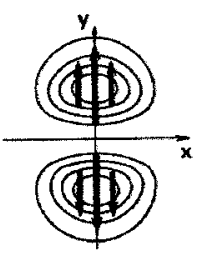

(d) Ex mode

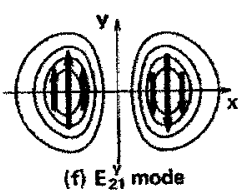

Figure 1.4: Mode definitions and electric field distributions in a strip waveguide. From [22].

the TE-like mode or $H_{z}$ of the TM-like mode is much weaker compared to the other field components.

A number of analysis techniques have been used to solve for waveguide modes, including the wave optics method, the matrix method, the modified ray model method, the finite difference method, the effective index method, and the finite element method $[14,22]$. The latter two approaches have been used for modal analysis in this project. The following two subsections describes the modal analysis for optical waveguides with ridge cross-sections defined in Fig. 1.3(b).

\subsubsection{Effective Index Method}

It is not trivial to solve for the exact field distributions of the optical modes in a threedimensional (3-D) waveguide. The effective index method (or EIM) [22] approximates a 3-D structure to a two-dimensional (2-D) slab waveguide problem. The EIM is known for its simplicity and accuracy, and has been widely and successfully used for ridge 
waveguides.

The EIM works by first decomposing the ridge waveguide cross-section into the core and slab regions as in Fig. 1.5(a), and then treating each region individually as an asymmetric slab waveguide to find their respective effective indices. To calculate the effective index of the "TE-like mode", $n_{\mathrm{eff}}^{\mathrm{TE}}$, solutions to the TE eigenvalue equation of an asymmetric slab waveguide are used in each of the three regions. The details of solving for effective index in a slab waveguide can be found in general optics books $[2,14,22-24]$, and hence, are not repeated here.
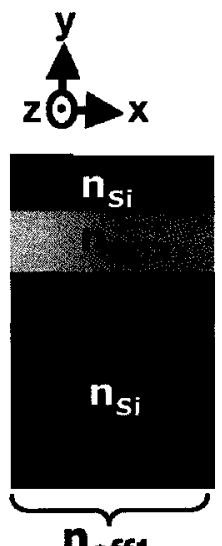

$\mathbf{n}_{\text {eff1 }}$

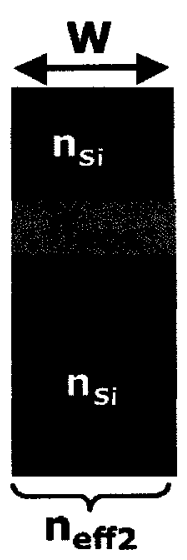

(a)

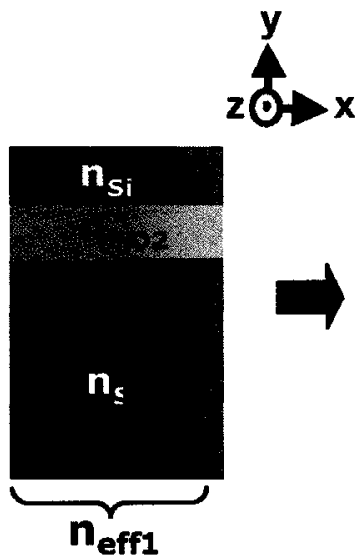

$\mathbf{n}_{\text {eff1 }}$

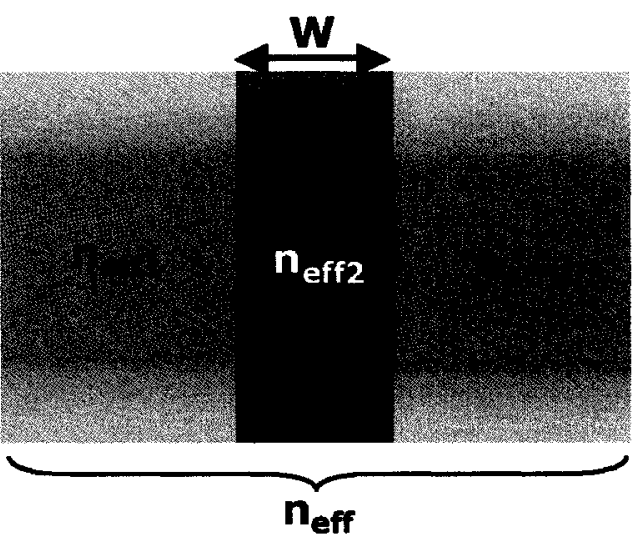

(b)

Figure 1.5: Effective index method (EIM): (a) a 3-D ridge waveguide is split into three parts: a core region and two cladding regions. Each region is treated as a 2-D slab waveguide to obtain $n_{\text {eff1 }}$ and $n_{\text {eff2 }}$. (b) The $n_{\text {eff1 }}$ and $n_{\text {eff2 }}$ are used to construct another three-layer slab waveguide to obtain the final effective index.

The resulting effective indices $\left(n_{\text {eff1 }}\right.$ and $\left.n_{\text {eff2 }}\right)$ are used to create an equivalent vertical three-layer slab waveguide, as shown in Fig. 1.5(b). This slab waveguide is oriented vertically instead of horizontally. To account for this change in orientation, the eigenvalue equation for TM should be used with $n_{\mathrm{eff} 1}^{\mathrm{TE}}$ and $n_{\mathrm{eff} 2}^{\mathrm{TE}}$ for deriving the overall effective index and the field distributions of the TE mode in a ridge waveguide.

Similarly, to solve for the effective index of the "TM-like mode" of a ridge waveguide, the effective indices for the TM modes are first calculated for the three regions defined in 
Fig. 1.5(a). A second slab waveguide, oriented vertically, is then constructed using the calculated $n_{\mathrm{eff} 1}^{\mathrm{TM}}$ and $n_{\mathrm{eff} 2}^{\mathrm{TM}}$. A TE eigenvalue equation should be used to obtain the final effective index of the TM mode in the ridge waveguide.

The EIM method performs best in low-index contrast materials and waveguide with simple geometrical cross-section profiles [2,22]. In many cases, ridge waveguides can have complicated cross-sections (with slanted ridge sidewalls, for example), which are difficult to model with the EIM.

\subsubsection{Finite Element Method}

In classical analytical procedures without subdivision processes, including the above mentioned EIM, a waveguide system is usually modeled using analytical procedures defined over the entire waveguide region [22]. Thus these procedures generally are applicable only to waveguides with simple geometries and material compositions. One of the simplest analytical method that employs the subdivision process is the finite different method (FDM), where the system under investigation is discretized into small regions using regular rectangular grids. However, waveguides with curved boundaries and interfaces cannot be accurately modeled by rectangular grids. The finite element method (FEM), on the other hand, analyzes the waveguide system by dividing the entire model domain into discrete elements. As a result, FEM is applicable to structures with arbitrary refractiveindex profiles and complicated cross-sectional geometries.

Mathematically FEM finds the approximate solution of partial differential equations derived from the wave equations by combining local solutions over discrete volume elements. In the following, FEM is used to analyze the propagation characteristics of a ridge waveguide shown in Fig. 1.3(b).

The starting assumption is that the electric field takes the form of

$$
\mathbf{E}(x, y, z)=\mathbf{E}(x, y) e^{-i \beta z}
$$


where $\beta$ is the propagation constant, and the propagation direction is along the $z$-axis. This assumption effectively reduces the $3-\mathrm{D}$ structure to a $2-\mathrm{D}$ waveguide problem. The vectorial wave equation can then be simplified and written in the form of [22],

$$
\nabla^{2} \mathbf{E}+\left[k^{2} \mathbf{n}^{2}(x, y)-\beta^{2}\right] \mathbf{E}(x, y)=0
$$

where $\nabla=\partial^{2} / \partial x^{2}+\partial^{2} / \partial y^{2}$ is the del operator, $k$ is the wave number $k=2 \pi / \lambda_{0}$, $\lambda_{0}$ is the vacuum wavelength, and $\mathbf{n}(x, y)$ is the index distribution of the waveguide. Instead of solving the wave equation directly, FEM transforms Eq. 1.2 into an equivalent variational problem with appropriate boundary conditions. The solution of the wave equation can be obtained by finding the $\mathbf{E}$ field that satisfies the stationary condition of the functional [22]:

$$
\Psi[\mathbf{E}]=\frac{1}{2} \int_{-\infty}^{\infty} \int_{-\infty}^{\infty}\left[\left(\frac{\partial \mathbf{E}}{\partial x}\right)^{2}+\left(\frac{\partial \mathbf{E}}{\partial y}\right)^{2}-\left(k^{2} \mathbf{n}^{2}-\beta^{2}\right) \mathbf{E}^{2}\right] d x d y
$$

The proof of the above solution can be found in [22], and will not be discussed here. The next step is the mesh generation, in which the entire structure is divided into $N$ discrete elements. Fig. 1.6 shows the domain of a sample ridge waveguide cross-section with $N=2752$ discretized triangular elements. The electric field $\mathbf{E}(x, y)$ in an element $i$ is approximated by a linear function of $x$ and $y$ :

$$
\mathbf{E}(x, y)^{i}=a_{0}^{i}+a_{1}^{i} x+a_{2}^{i} y
$$

where $a_{0}^{i}, a_{1}^{i}, a_{2}^{i}$ are constants for the element $i$, and can be determined based on the properties of the specific element. Each element is triangular in shape, defined by three nodal points. The electric field amplitude at each nodal point shares the same linear function as in Eq. 1.4, with the appropriate $x$ and $y$. The expression in Eq. 1.3 is evaluated with the electric field defined in Eq. 1.4 for each discretized element. The overall solution (i.e., the electric field distribution and the propagation constant) of the entire domain is formed by the summation of the individual elements; that is,

$$
\Psi=\sum_{i=1}^{N} \Psi^{i}
$$




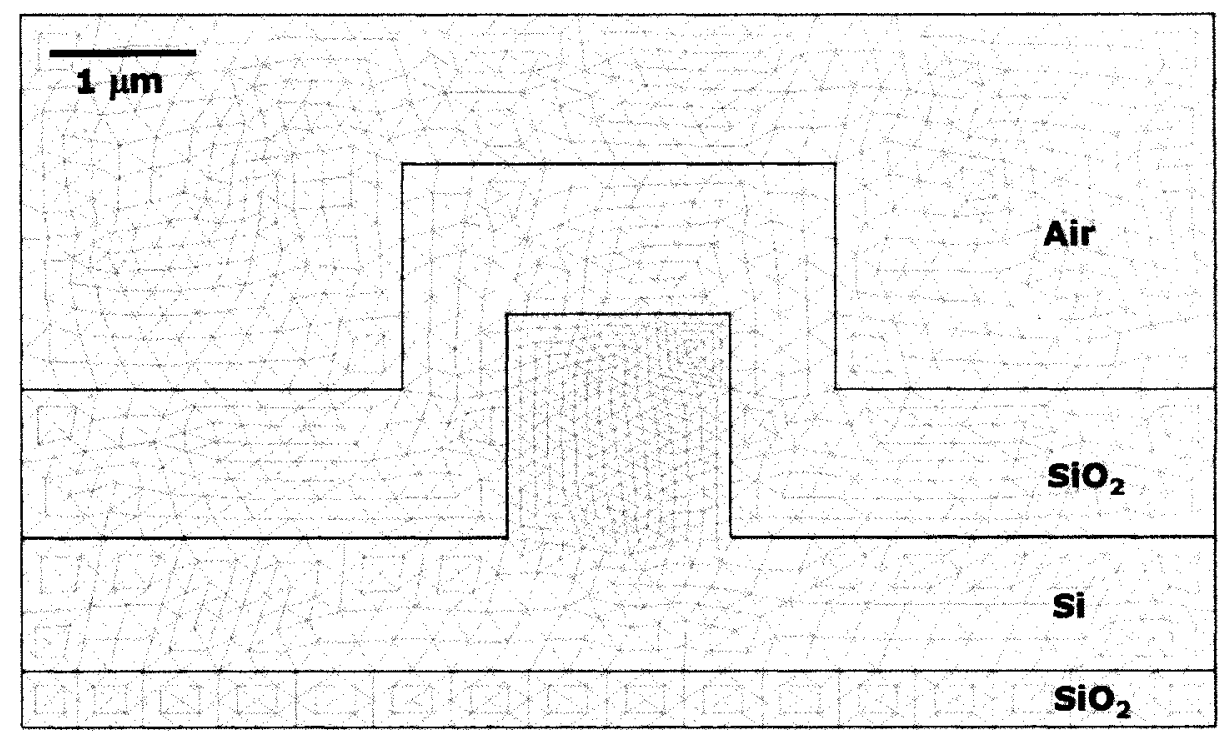

Figure 1.6: Examples of element discretization in FEM waveguide analysis. The number of mesh elements is $N=2752$.

The effective index $\left(n_{\text {eff }}\right)$ of the modes in the waveguide are then determined for both the TE- and TM-like polarizations, by following the relation $n_{\text {eff }}=\beta \lambda_{0} /(2 \pi)$. The total number of the mesh elements, or mesh density, is crucial to the accuracy of the solution. Usually the accuracy of the solution is improved by simply increasing the number of mesh elements in regions where $\mathbf{E}$ is changing rapidly. However, this may result in a time-consuming calculation process. A convergence analysis is generally performed to determine an appropriate mesh density required to achieve acceptable accuracy.

\subsubsection{Single Mode Condition}

In the previous sections, we discussed the numerical methods to calculate the field distributions of optical modes in a ridge waveguide. The total number of optical modes that is supported by a waveguide depends on its ridge cross-section geometry. In most of the sensor and telecommunication applications, waveguides are required to support a single mode, which means only the fundamental mode is allowed to propagate in the waveguide. It is worth emphasizing that the term "single mode" implies that the lowest order orthogonally polarized TE and TM waves are supported. 
Fig. 1.7 shows the geometry of a typical SOI ridge waveguide with a $\mathrm{SiO}_{2}$ upper cladding layer, similar to those we investigate in this work. The silicon ridge width $W$, etch depth $D$, and the total core thickness $H$ define the three basic ridge waveguide dimensions. The wide use of ridge waveguide geometry is due to the fact that the waveguides can be designed to support only the fundamental mode, even if the slab waveguides with the same core thickness are multimode.

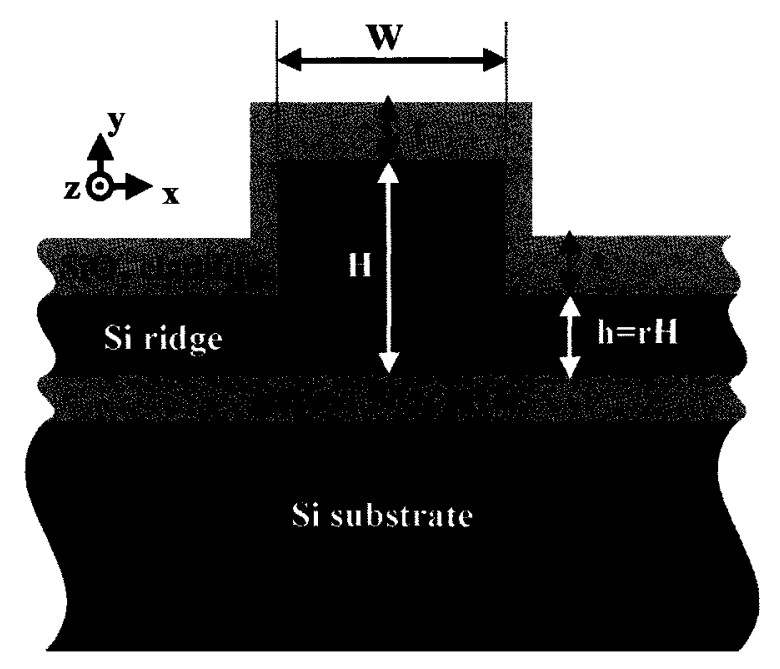

Figure 1.7: The cross-section of a ridge waveguide in an SOI platform: geometry parameter definitions.

Soref et al. [20] were the first to propose that a ridge structure with large crosssectional dimensions of several microns could guide only the fundamental mode. The higher-order modes do exist initially in the light propagation; however, with appropriate design of the cross-section geometry, all higher-order modes are leaky and only the fundamental mode in the central ridge region survives after a short propagation distance [20]. With the geometry parameters defined in Fig. 1.7, the approximate expression of the single-mode condition for relatively large SOI ridge waveguides was derived by Soref et al. to be:

$$
\frac{W}{H} \leq 0.3+\frac{r}{\sqrt{1-r^{2}}}, \quad \text { for } r \geq 0.5
$$

where $r=h / H$ is the ratio of the slab height $(h)$ to the Si ridge height $(H)$. The restriction imposed by $r \geq 0.5$ ensures that all the higher-order vertical modes in the 
central ridge region will leak out of the waveguide, leaving only the fundamental mode in the waveguide. For waveguides that satisfy Eq. 1.6, simulations have verified that all the higher-order vertical modes, except the fundamental mode in the central ridge region have propagation constants lower than that of the fundamental mode of the slab waveguide in the side regions. Thus the higher-order modes will couple into the adjacent slab regions, and experience a high propagation loss. The waveguide behaves as a single mode waveguide as all higher-order modes are lost during propagation.

The single-mode conditions were studied experimentally by Rickman et al. [25]. The experimental data was further analyzed with the effective index method (EIM) by Pogossian et al. [21]. A constant of -0.05 instead of the 0.3 in Eq. 1.6 was claimed to fit better with the experimental data. Fig. 1.8 [21] summarizes the theoretical calculations and experimental results of the single mode condition in ridge waveguide with relatively large cross-sections.

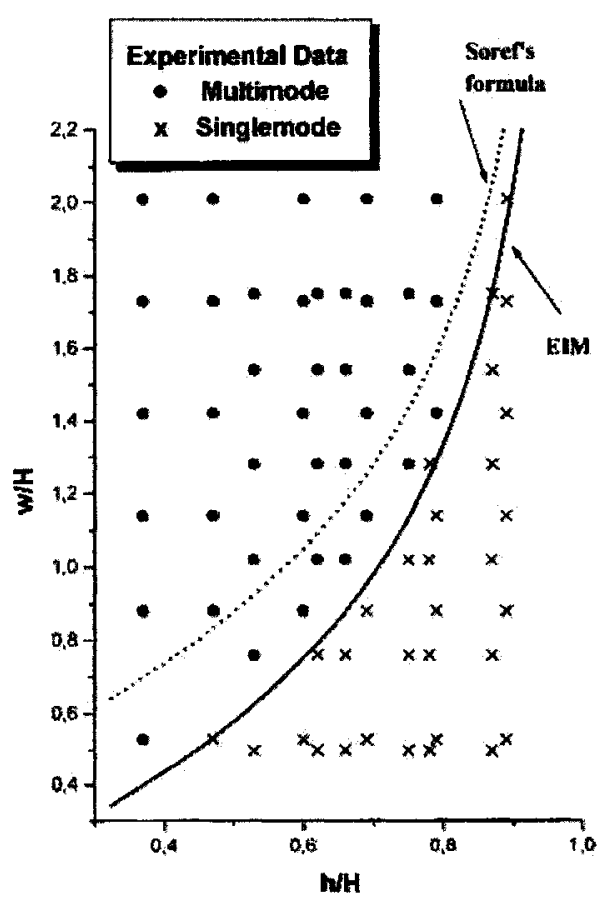

Figure 1.8: The single mode condition for SOI ridge waveguides: dotted curve (Eq. 1.6), solid curve (Eq. 1.6 with a modified -0.05 constant), and single and multimode experimental data points. From [21]. 
Soon after, Powell [26] published his work verifying the single mode condition by using the beam propagation method. The new simulation results confirmed the original condition defined in the empirical equation (Eq. 1.6), and with a slight width $(W)$ variation in Eq. 1.6, this single mode condition was claimed to be applicable to ridge waveguides with both vertical and angled sidewalls. Similar to previous investigations, the analysis was limited to waveguides of large ridge cross-section with shallow etch depth $(r \geq 0.5)$.

Recently, Chan et al. [27] from University of Surrey reported their study on the polarization characteristics of small waveguides ( $\sim 1 \mu \mathrm{m}$ or less in cross-section) with deeply etched ridges. The single mode condition was generalized as:

$$
\frac{W}{H} \leq 0.05+\frac{(0.94+0.25 H) r}{\sqrt{1-r^{2}}}, \quad \text { for } 0.3<r<0.5 \text { and } 1.0 \leq H \leq 0.5
$$

The relation defined in Eq. 1.7 is similar to Soref's Eq. 1.6, with only small changes to the terms.

In summary, the conditions listed in Eq. 1.6 and Eq. 1.7 are empirical relations that apply over a range of waveguide dimensions. However, they are not fundamental conditions for single mode waveguides, and do not apply to all situations. Soref's Eq. 1.6 has been generally accepted as a guideline for the choices of the available ridge width and etch depth ratios in keeping the waveguide single mode. Commercial mode solvers are usually used to simulate the mode characteristics and verify the single mode design of a particular waveguide geometry.

\subsubsection{Waveguide Birefringence}

As discussed in the previous section, the fundamental mode contains two orthogonally polarized components, the TE and TM modes. For a perfectly symmetric waveguide (e.g., square), the propagation constants of the two orthogonal components are identical due to the identical boundary conditions imposed by the geometry. However, waveguides are rarely perfectly symmetric, and even in the case where the geometry is designed to be 
symmetric, external factors such as fabrication errors, temperature fluctuations, or stress will always cause a difference in the propagation constants or the effective indices (i.e., $\left.\beta=2 \pi n_{\text {eff }} / \lambda\right)$ of the TE and TM modes. This difference between the effective indices of these two polarized modes results in waveguide birefringence.

In a typical SOI ridge waveguide presented in Fig. 1.7, waveguide birefringence is defined as the difference between the effective indices of the out-of-plane polarized TM mode (with the electric field along the $y$-axis) and the in-plane polarized TE mode (with the electric field along the $x$-axis); that is, $\Delta n_{\text {eff }}=n_{\text {eff }}^{\mathrm{TM}}-n_{\mathrm{eff}}^{\mathrm{TE}}$. Fig. 1.9 illustrates the electric field profiles of the TE- and TM-polarized mode of a typical SOI ridge waveguide. The electric field distributions of the TE and TM modes satisfy the boundary conditions

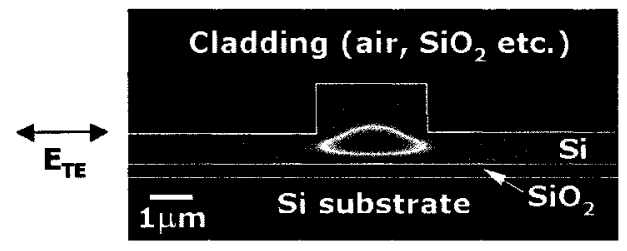

(a)

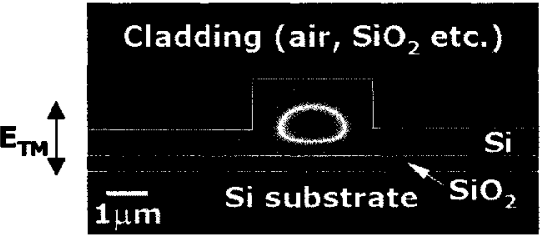

(b)

Figure 1.9: The electric field profiles of the (a) TE-polarized and (b) the TM-polarized mode of a typical SOI ridge waveguide. The waveguide has a ridge width of $2.5 \mu \mathrm{m}$, an etch depth of $1.5 \mu \mathrm{m}$, and a ridge height of $2.2 \mu \mathrm{m}$.

at the interface between two different material layers. In the absence of surface electric charge and current density, the tangential components of the electric and magnetic field, and the normal components of the electric displacement $\mathbf{D}=\epsilon \mathbf{E}$ and the magnetic induction $\mathbf{B}=\mu \mathbf{H}$, are continuous across the boundary interface; that is,

$$
\mathbf{E}_{t}^{a}=\mathbf{E}_{t}^{b} ; \quad \mathbf{H}_{t}^{a}=\mathbf{H}_{t}^{b} ; \quad \mathbf{D}_{n}^{a}=\mathbf{D}_{n}^{b} ; \quad \mathbf{B}_{n}^{a}=\mathbf{B}_{n}^{b}
$$

where the subscripts $t$ and $n$ denote the tangential and normal components to the boundary interface, respectively, the superscripts $a$ and $b$ indicate the two media, and $\epsilon$ and $\mu$ are the permittivity and permeability of the medium, respectively. In addition, there is a natural boundary condition that requires the electromagnetic fields to be zero at infinity. The high index contrast of the core and cladding layer in an SOI system gives a large 
discontinuity in $\epsilon$ at the layer interface. Thus the TE mode properties can significantly differ from those of the TM, leading to a non-zero birefringence, as shown in Fig. 1.9.

Waveguide birefringence $\left(\Delta n_{\text {eff }}\right)$ consists of two types, geometrical birefringence $\left(\Delta n_{\text {geo }}\right)$ and stress-induced birefringence $\left(\Delta n_{\text {stress }}\right): \Delta n_{\text {eff }}=\Delta n_{\text {geo }}+\Delta n_{\text {stress. }}$. The former is the consequence of an axially asymmetric waveguide cross-section [22] (as shown in Fig. 1.9), while the latter is produced by the anisotropic stress distributions in the waveguide [28].

Birefringence is an important issue in integrated optical systems. It gives rise to the polarization mode dispersion (PMD) in optical fibers, the polarization dependent modulation (PDM) in electro-optic modulators, the polarization dependent gain (PDG) in optical amplifiers, the polarization dependent center wavelength $(\mathrm{PD} \lambda)$ in WDM filters, the polarization dependent response (PDR) in receivers, and the polarization dependent sensitivity (PDS) in sensors and coherent communication systems $[14,22,29]$. Hence, polarization management is crucial to the design, implementation, and operation of optical devices.

Two schemes have been proposed and implemented for polarization management: one is to make devices polarization insensitive, and the other is to use the polarization diversity approach [23]. The former method requires a precise polarization independent design of the waveguide device, while the latter method relies on a polarization splitter device to separate the TE and TM from each other and process them individually to avoid the birefringence problem. Although in principle the birefringence-free and single-mode waveguides with large cross-sections can be achieved for waveguides by adjusting the core geometry [30], our previous studies [31,32] have shown that this approach is not practical for waveguides with dimensions on the order of a micron. In addition, the waveguide core geometry determines other critical performance parameters such as the mode sizes, minimum allowed bend radius, and the coupling between adjacent waveguides. Furthermore, the low tolerance to dimensional fluctuations arising from fabrication processes poses challenging technical problems. These challenges, limitations and constraints motivate 
research into exploring novel, effective, and practical methods for polarization management, which is the primary focus of this thesis research project.

\subsection{Thesis Organization}

The organization of the thesis is as follows:

Chapter 2 "Literature Review and Thesis Objective" first reviews the recent advances in polarization management by comparing various methods reported in the literature for slica-based, III-V compound semiconductor, and SOI-based planar waveguide devices. The advantages and disadvantages of these polarization control methods are discussed systematically. Most of these methods have been developed specifically for silica-onsilicon based waveguide devices. Limited work has been targeted for efficient and practical polarization control in high-index-contrast systems such as SOI. The urgent need for viable microphotonic polarization control devices requires an effective and practical method. The objective of the research project is then identified at the end of the chapter.

Chapter 3 "Stress: Theory and Modeling" begins with a review on the concept of the stress and the photoelastic relations, followed by descriptions of the available numerical analysis techniques for modeling stress. After presenting the issues related to these available techniques, a simple and accurate calculation scheme, the "Normalized Plane Strain Model", is proposed and derived. Based on this numerical model, a comprehensive study of the stress-induced effects from the cladding layer, combined with the geometrical contribution, on the overall effective index and birefringence are carried out in Chapter 4 "Stress Engineering - Stress and Geometry Induced Effects". The effects of the sidewall angle, width, and etch depth of the ridge waveguides, combined with the stress effects, are investigated systematically. After that, an experimental demonstration of stress engineering in polarization management in SOI waveguide devices is presented in Chapter 5 “Experimental Demonstration of Stress Engineering”. A polarization insensitive arrayed 
waveguide grating is reported using stress compensation. The experimental results not only validate our predictions of the stress effects on birefringence, but also confirm the usefulness, practicality, and effectiveness of the approach.

An important application of stress engineering is in passive polarization splitters and filters. A number of novel passive polarization splitters and filters utilizing stress engineering are proposed. The designs are mainly focused on two configurations: the MachZehnder interferometer (MZI) and the zero-order arrayed waveguide grating (AWG), presented in Chapter 6 "Stress-induced MZI-based Polarization Splitters/Filters" and Chapter 7 "Stress-induced Zero-order AWG Polarization Splitters", respectively. Both chapters explain the actual design options, modeling, fabrication, and characterization of the devices. They start with the device operating principles, followed by the design considerations in terms of the ridge cross-section geometry, temperature sensitivity, and dispersion effects, with supporting simulation results. Then the optical mask layout is explained, with a detailed description of the fabrication flow. Finally, the performance of the fabricated test devices is investigated in detail, by comparing with the numerical simulated results.

Finally, the thesis ends in Chapter 8 "Conclusions" with an overview of the significant contributions of this thesis project made to the field of polarization management in optical waveguide devices. Remarks on the potential improvements to the device design and fabrication and suggestions of future research directions are included. 


\section{Chapter 2}

\section{Literature Review and Thesis}

\section{Objective}

The chapter begins with a detailed survey of past and present research on the subject of polarization control in planar optical waveguide devices, followed by the objective of the thesis project. The aim of this chapter is to lay a foundation for the remainder of the thesis by identifying what has been done, and propose a feasible scheme to fill the knowledge gap identified in the published literature.

\section{$2.1 \quad$ Past Research}

The performance of a waveguide device should ideally be the same regardless of the polarization. However, optical devices usually have different effective indices for the two orthogonally polarized waveguide modes: the transverse electric (TE) mode with the electric field polarized parallel to the wafer plane and the transverse magnetic (TM) mode with the electric filed polarized in the normal direction of the wafer plane. This property is referred to as the "waveguide birefringence". It almost always exists in waveguide devices, with only a few exceptions such as perfectly symmetric waveguides, where the two orthogonally polarized modes are degenerate. Birefringence is in general undesir- 
able as it degrades the device performance and causes unwanted polarization dependent wavelength shift and phase shift in all interferometric devices such as spectrometers, Mach-Zehnder interferometers, and ring resonators. In applications such as wavelength demultiplexing and high-resolution spectroscopy, birefringence results in a wavelength shift in the spectral response peak of each wavelength channel, which limits the wavelength resolution and affects the spectral channel density. A birefringence less than $10^{-5}$, which corresponds to a polarization dependent wavelength shift in the center wavelength of $\Delta \lambda<0.04 \mathrm{~nm}$, is usually required for acceptable polarization insensitive spectrometers. Clearly, polarization control and manipulation are a crucial element in the design and operation of optical devices.

Two approaches have been attempted to mitigate the birefringence related problems in planar integrated optics: one is to produce polarization insensitive devices by birefringence elimination or compensation, and the other is to separate the TE and TM polarized light and process each polarization individually by polarization splitting. Both approaches have been demonstrated with different degree of success in the control and management of polarization dependence in devices fabricated in silica-based waveguides, III-V semiconductors, and silicon-on-insulator (SOI). A detailed review on these research activities are presented in the next two sections.

\subsubsection{Birefringence Elimination}

In this polarization control method, device birefringence is completely eliminated by either the design of the waveguide structures or the insertion of polarization compensation components. Consequently, the polarization sensitivity is effectively controlled or removed in device performance. Past research on polarization insensitive planar waveguide devices are based on three main types of material systems: silica-on-silicon, III-V semiconductor, and SOI. 


\section{Silica Waveguide Devices}

In silica planar waveguides such as silica-on-silicon devices (shown in Fig. 2.1), the index step between the core and the cladding is typically about $1 \%$. The geometrical contribution to the waveguide birefringence is negligible due to the large core size and the low index contrast. The birefringence is primarily due to the biaxial stress distribution caused by the large mismatch in the thermal expansion coefficients between the constituent silica layers and the silicon substrate. This stress-induced birefringence ranges from $\Delta n=10^{-4}$ to $10^{-3}$, depending on the material composition, deposition conditions, and processing [33]. An enormous amount of research has been devoted to reduce and elimi-nate the stress in achieving polarization insensitive operations for silica based devices.

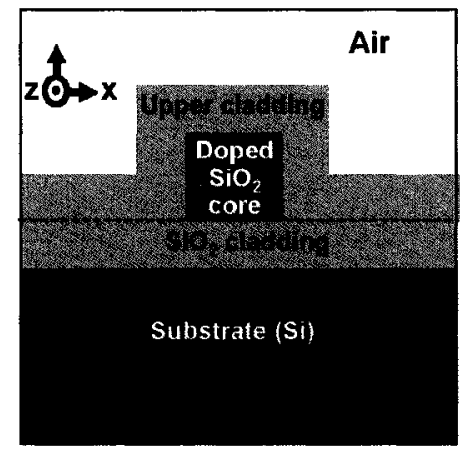

Figure 2.1: Cross-section of a silica-on-silicon planar waveguide.

The arrayed waveguide grating (AWG) based devices have been widely investigated as waveguide (de)multiplexers for wavelength division multiplexing (WDM) systems. Polarization sensitivity is one of the key problems of these devices. Several schemes have been developed for making polarization insensitive AWGs. One way to avoid the stressinduced effects is to use a silica substrate, instead of silicon [34]. Polarization insensitive AWG devices fabricated on $\mathrm{SiO}_{2}-\mathrm{On}-\mathrm{SiO}_{2}$ were demonstrated [34-36]. Alternatively, a dopant-rich cladding whose thermal expansion coefficient is similar to that of the Si substrate can be applied to balance the internal stress in the waveguide [37]. The effects 
of the cladding dopants such as phosphorus and boron on the thermal coefficient of the cladding layer and the fabrication deposition processes with precise dopant concentration control have been studied extensively. Numerous polarization insensitive AWG devices with dopant-rich cladding layer have been reported [37-40].

The insertion of a half-wave $(\lambda / 2)$ plate at the middle point of an AWG structure was also proven to be useful for birefringence elimination [41,42]. Fig. 2.2 presents a schematic diagram of a polarization insensitive AWG with a $\lambda / 2$ plate inserted in the center of the arrayed waveguide section. A $\lambda / 2$ plate can rotate the polarization direction from $\mathrm{TE}$ to

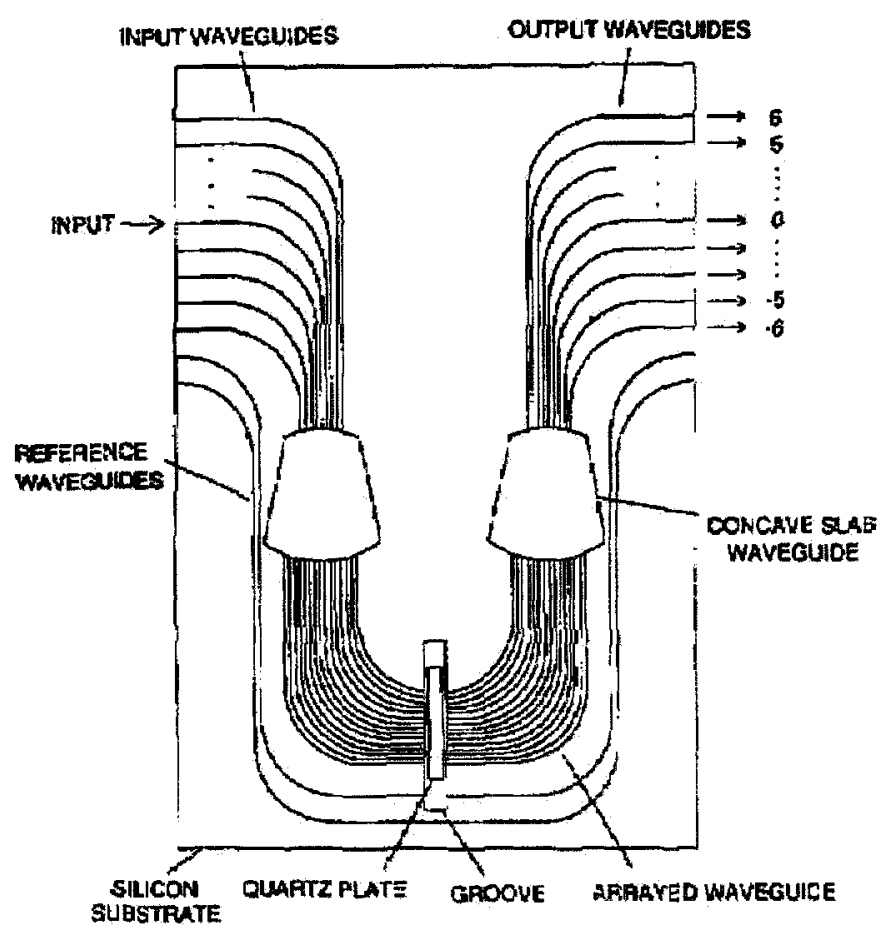

Figure 2.2: Schematic layout of a polarization insensitive arrayed waveguide grating device fabricated using $\mathrm{SiO}_{2}-\mathrm{GeO}_{2} / \mathrm{SiO}_{2}$ on a $\mathrm{Si}$ substrate. A halfwave plate is inserted in the center of the grating section. From [41].

TM and vice versa. In other words, light entering the AWG as the TE (or TM) polarized light propagates through the first half of the device as the TE (or TM) polarization, and the second half of the device as the TM (or TE) polarization. The interchanging of the polarization states from the $\lambda / 2$ plate effectively removes the polarization dependence of the center wavelength. The authors demonstrated the elimination of a birefringence of 
$3.3 \times 10^{-4}$ in the AWG device by placing a $\lambda / 2$ plate into a $100 \times 500 \mu \mathrm{m}^{2}$ groove in the center of the arrayed grating section.

Soon after, the same research team, led by Takahashi et al. [43], reported that the deposition of an amorphous silicon film (a-Si) on selected areas of the waveguides could compensate birefringence. The residual stress in the a-Si film compensates the initial stress that existed in the waveguide device. This method was introduced in the demonstration of a polarization insensitive AWG device, as illustrated in Fig. 2.3. The birefringence-free

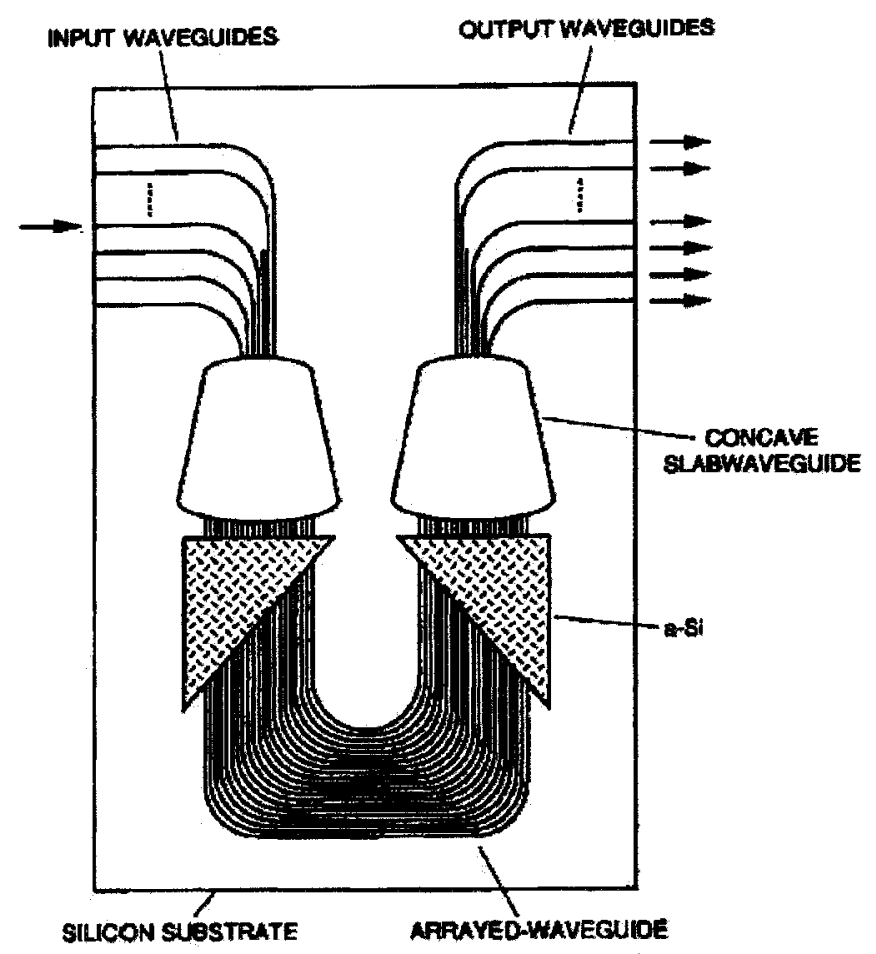

Figure 2.3: Schematic of the arrayed waveguide multiplexer. The shaded patch is the amorphous Si film (a-Si) for stress compensation. From [43]

condition is defined as:

$$
\left(n_{\mathrm{TM}}-n_{\mathrm{TE}}\right)(\Delta L-\Delta l)+\left(n_{\mathrm{TM}}^{\prime}-n_{\mathrm{TE}}^{\prime}\right) \Delta l=0
$$

where $n^{\prime}$ and $n$ denote the polarization dependent effective index with and without the a-Si film, respectively, $\Delta L$ is the waveguide length increment in the arrayed grating section, and $\Delta l$ is the patch length increment between two adjacent waveguides in the a-Si film. Two symmetric patches of $8 \mu \mathrm{m}$ thick a-Si film with an area of $7.5 \times 10^{5} \mu \mathrm{m}^{2}$ 
were deposited to achieve a stress-induced birefringence modification of $4.4 \times 10^{-5}$. The measured polarization dependent wavelength shift was reduced from $0.11 \mathrm{~nm}$ to less than $0.06 \mathrm{~nm}$.

A further method for achieving polarization insensitivity in silica planar waveguides is the use of stress-relief grooves in close proximity to the waveguides [44, 45]. Fig. 2.4 shows a polarization insensitive AWG device with stress relief grooves. By placing the stress relief grooves close to the arrayed waveguides, the internal stress in the waveguides can be effectively released. The grooves had an etch depth of $d_{\text {etch }}=5.8 \mu \mathrm{m}$ and the gap

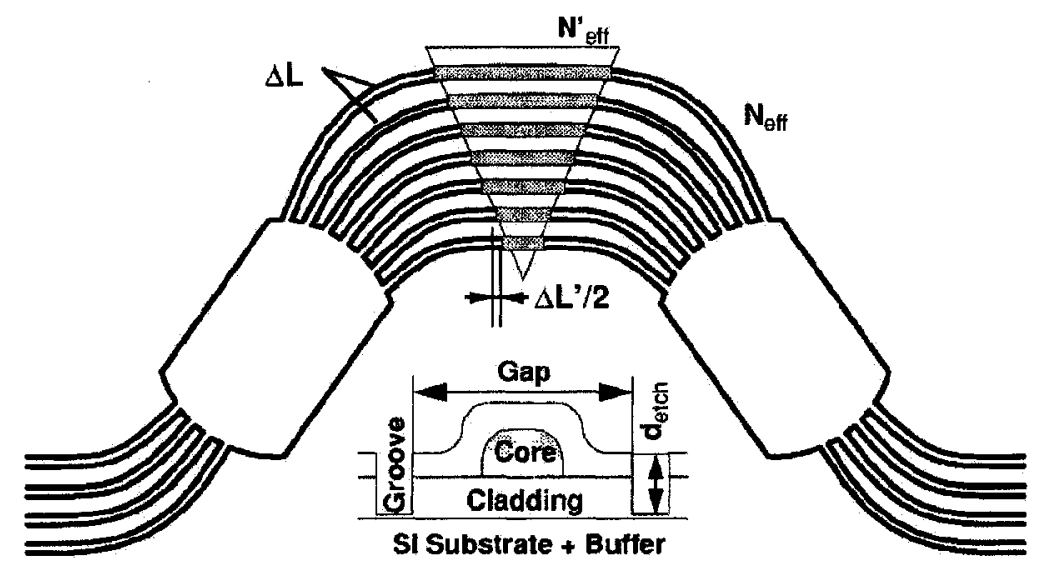

Figure 2.4: Schematic of an arrayed waveguide grating device with stress relief grooves for birefringence compensation. From [45]. The $\mathrm{SiO}_{2}$-on-Si waveguide cross-section is also shown in the inset diagram. The groove had an etch depth of $d_{\text {etch }}=5.8 \mu \mathrm{m}$ and a width of $75 \mu \mathrm{m}$, the gap increment between two adjacent arrayed waveguides was $36 \mu \mathrm{m}$.

increment between two adjacent waveguides in the arrayed grating section was $36 \mu \mathrm{m}$. The authors reported that the grooves reduced the polarization shift of the AWG from $0.66 \mathrm{~nm}$ to less than $0.05 \mathrm{~nm}$. This corresponds to an order of magnitude decrease in birefringence (from $6 \times 10^{-4}$ down to $5 \times 10^{-5}$ ). The 16-channel $200 \mathrm{GHz}$ AWG device has a chip size of $60 \times 20 \mathrm{~mm}^{2}$.

The polarization control methods mentioned thus far have been focused on the AWG demultiplexers. Another type of integrated waveguide (de)multiplexer is based on a diffraction grating. A diffraction grating consists of a surface with many parallel grooves, 
which disperse light into spatially separated wavelength components. Besides the perceived difficulties in manufacturing planar waveguide diffraction grating devices, polarization dependence was a fundamental challenge in implementing a commercially viable diffraction grating technology [33]. Janz et al. developed a polarization compensator to eliminate the TE-TM shift in channel output wavelength in an etched diffraction grating demultiplexer fabricated on a silica-on-silicon waveguide platform [33]. The device consists of input/output waveguide arrays, a slab waveguide region, and an etched diffraction grating, as shown in Fig. 2.5. Here the device birefringence mainly comes from the slab

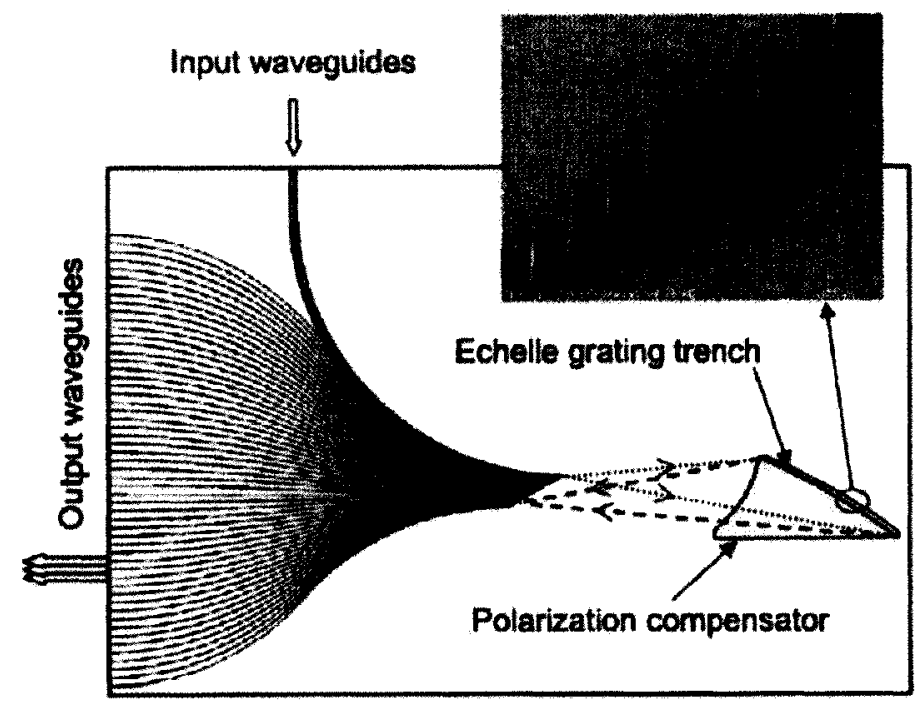

Figure 2.5: Schematic layout of a 48-channel diffraction grating based waveguide demultiplexer with a polarization compensator. Inset: an SEM image of the etched diffraction grating. From [33].

region. The birefringence modification was realized by etching the slab waveguide section to define a triangular shaped polarization compensator, with a thin nitride layer deposited on top. The TE and TM polarized light are diffracted at slight different angles after passing through the compensator such that they both focus at the same focal point on the grating. This method was originally proposed and implemented in an InP-based grating spectrometer [46], which will be discussed shortly. The grating-based demultiplexer in the silica-on-silicon platform with an etched polarization compensator was demonstrated with a polarization dependent wavelength shift of $0.01 \mathrm{~nm}$ which corresponded to a total 
waveguide birefringence of less than $1 \times 10^{-5}$. The 48-channel spectrometer with a 100 $\mathrm{GHz}$ channel spacing has an overall chip size of $18 \times 17 \mathrm{~mm}^{2}$. The scalability of this type of device was also demonstrated. No significant increase in the chip size was observed by scaling the total channel counts to 256 channels. A comparable 256 channel AWG device, on the other hand, would take up about five times the area of the grating-based chip [33].

Laser processing offers another way to tailor and tune birefringence in silica-onsilicon based waveguides. Albert et al. [47] first demonstrated polarization insensitive ultraviolet-induced Bragg gratings in silica-on-silicon planar waveguides. The ultraviolet (UV) irradiation introduces damage at the silica/silicon interface which relaxes the internal stress in the waveguide through a reduction of the adhesion between the substrate and the silica. Intense ArF excimer laser light at $193 \mathrm{~nm}$ at a pulse repetition rate of 100 pulse per second was used to form the gratings [47]. The UV-induced birefringence of more than $3 \times 10^{-4}$ is used to compensate for the inherent birefringence of the germanium-doped silica-on-silicon waveguides. The Bragg grating response was identical for TE and TM modes, with the polarization dependent Bragg wavelength shift reduced from $0.2 \mathrm{~nm}$ to less than $0.05 \mathrm{~nm}$. Further studies on UV irradiation $[48,49]$ and mid-infrared laser processing [50] on birefringence compensation were also carried out. Based on this technology, a compact optical add/drop multiplexer (Fig. 2.6), with the polarization independent Bragg gratings written on the two arms of a symmetric MachZehnder interferometer (MZI), was demonstrated [51]. Two identical Bragg gratings of $3.8 \mathrm{~mm}$ in length were written on the two arms of the MZI. The imbalance in the two Bragg gra-tings and the two MZI arms was compensated by uniform UV trimming. The add/drop function can be described as follows. In the drop mode, all the light entering Port 1 exits at Port 3, except for the light resonant with the Bragg wavelength which exits at Port 4. Due to the symmetry of the device, light at the Bragg wavelength can be added to the light going out of Port 3 by injecting it in Port 2. The UV-induced birefrin- 


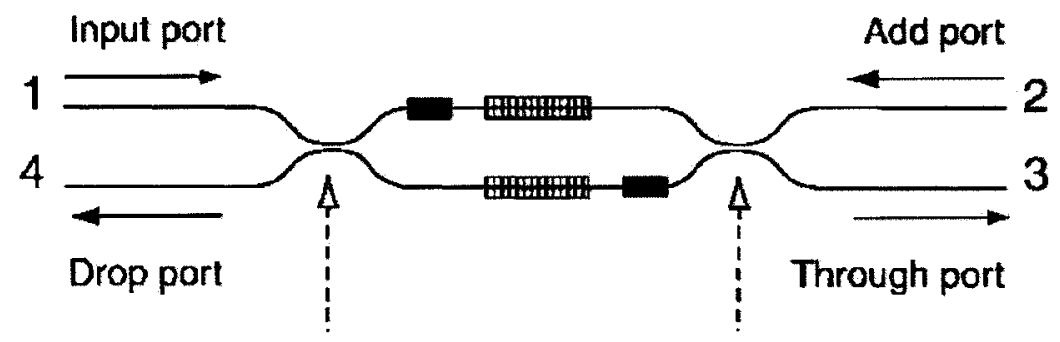

\section{Broadband $3 \mathrm{~dB}$ couplers}

Figure 2.6: Schematic layout of a planar waveguide balanced MachZehnder interferometer. The device length is $25 \mathrm{~mm}$ with a $3.8 \mathrm{~mm}$-long Bragg grating in each arm. From [51].

gence compensation ensures the polarization insensitivity in the device response. One of the main advantages of this method is that the UV-induced birefringence is produced automatically during the Bragg grating formation. However, the high UV exposure dosage may result in undesirable damage to the waveguide device.

\section{III-V Semiconductor Devices}

Birefringence control has also been investigated for III-V semiconductor devices. Unlike the silica-based devices, the birefringence in III-V semiconductors is significantly dependent on the geometry of the waveguides. Thus, most of the birefringence control methods involve specific designs of the waveguide geometry. For example, Bissessur et al. studied a special layered InGaAsP/InP structure to achieve low device birefringence [52]. An AWG demultiplexer fabricated on InP with the proposed layered guiding core was demonstrated to have a polarization dependent wavelength shift of less than $0.01 \mathrm{~nm}$ which corresponded to a birefringence of $1 \times 10^{-5}$. However, the low-index contrast of the layered core causes the low confinement of the mode. Large bending radius is necessary to prevent significant radiation losses for the AWG device. The overall chip size of the 16-channel AWG device with 48 arrayed waveguides is $33.5 \times 4.2 \mathrm{~mm}^{2}$. The same research group later investigated a "raised strip" structure to achieve zero birefringence in waveguides, which is illustrated in the inset of Fig. 2.7. The InGaAsP core layer was 


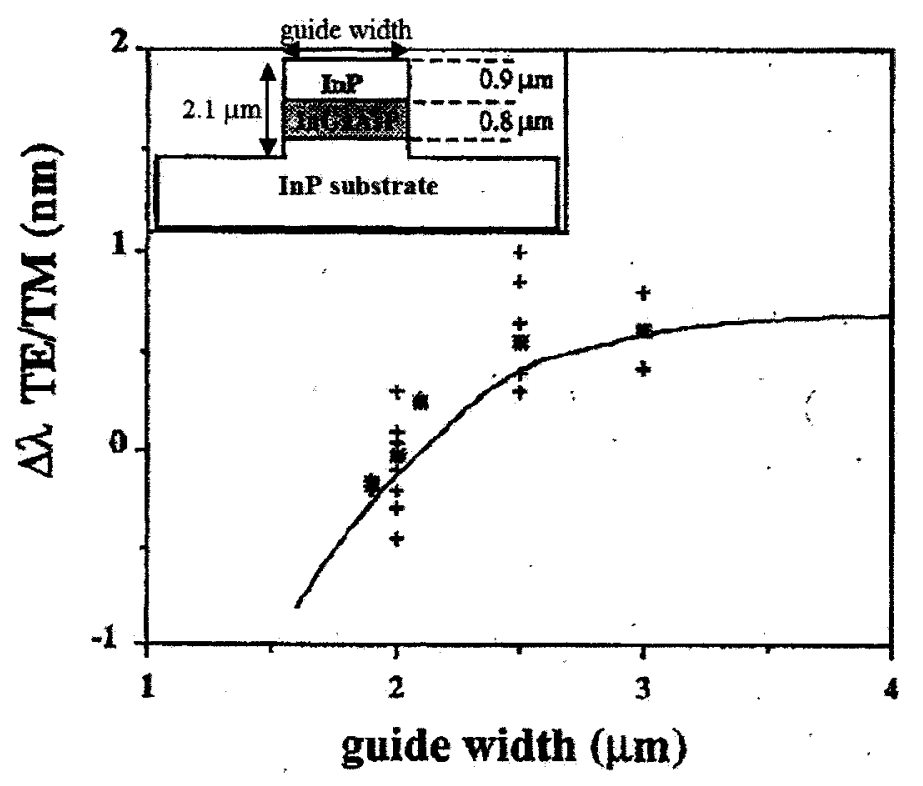

Figure 2.7: Birefringence as a function of waveguide width. The guiding structure was InGaAsP. Experimental results (data points) were compared with simulation results to determine the width of the strip waveguide. From [53].

over-etched down to the InP substrate by $0.4 \mu \mathrm{m}$. The over-etch of the lower cladding compensates and eliminates the birefringence of the waveguide structure [53]. Fig. 2.7 shows that a $2 \mu \mathrm{m}$ waveguide width fulfills the birefringence-free condition. The deep etch ridge structure allows the bending curvature to be less than $100 \mu \mathrm{m}$. A 16-channel polarization independent AWG device with 80 arrayed waveguides has a chip size of $1.6 \times 1.7 \mathrm{~mm}^{2}$, which is much smaller than their earlier work presented in Ref. [52] and is orders of magnitude smaller than the silica-based AWG devices [41,43,45]. The "raised strip" approach has also been adopted to silica-on-silicon waveguide design [54].

Soole et al. [55] reported the use of non-birefringent waveguides with square crosssection in a demonstration of a polarization independent InP-based AWG device. The square geometry ensures birefringence-free operation as well as the polarization independent loss. The InGaAsP waveguide core was designed to have a cross-section dimension of $1.8 \times 1.8 \mu \mathrm{m}^{2}$. The average measured shifts between the TE and TM were below 0.008 $\mathrm{nm}$ for all the wavelength channels. 
Designs of matching the free spectral range (FSR) and the polarization shift of the multiplexer were also reported for achieving polarization insensitive response in AWG devices [56]. FSR is defined as the wavelength separation between adjacent transmission peaks. To match a polarization wavelength shift of $\Delta \lambda_{\mathrm{TE}-\mathrm{TM}}=4.7 \mathrm{~nm}$ which corresponds to a birefringence of $1 \times 10^{-2}$, the grating order of the AWG for the TE mode was designed to be exactly one order higher than that for the TM at the same wavelength; that is, the spectral patterns of $\mathrm{TE}_{m}$ and the $\mathrm{TM}_{m-1}$ overlap. The disadvantage of this method is that the spectral width of the device is restricted to be within the range of the TETM wavelength shift, which limits the number of channels and/or the channel spacing, resulting in a relatively narrow operating bandwidth.

Birefringence compensation using patch segments of different lengths in the waveguide array were proposed and demonstrated in InP-based AWG devices [57]. For example, in Fig. 2.8, a section of straight waveguides in the middle of the AWG, marked as the triangular shaped patch, is specially designed for birefringence control. Outside of the patched area, the InGaAsP waveguiding cores were buried at $1 \mu \mathrm{m}$ below the top surface of the InP cladding. In contrast, the cladding for the waveguides in the patch segments was thinner, with a thickness of around $0.2 \mu \mathrm{m}$. With this method, the TE and TM polarized beams experience different refractive index in the patched and normal sections of the arrayed waveguides. A birefringence-free operation was successfully demonstrated.

For diffraction grating based demultiplexers, the elimination of the polarization dependence can be accomplished by exploiting the low polarization-dependent slab waveguide between the input/output waveguides and the grating. Gini et al. [58] obtained low index contrast and birefringence-free slab waveguides using homogeneous $\operatorname{InP}$ with different doping levels for the guiding and the cladding layers. The design restrictions on the layered structures pose severe limitations for this type of integrated grating demultiplexers. J.-J. He et al. proposed and implemented a polarization dispersion compensator in an InP-based grating demultiplexer [46], where the compensator was formed by selectively 


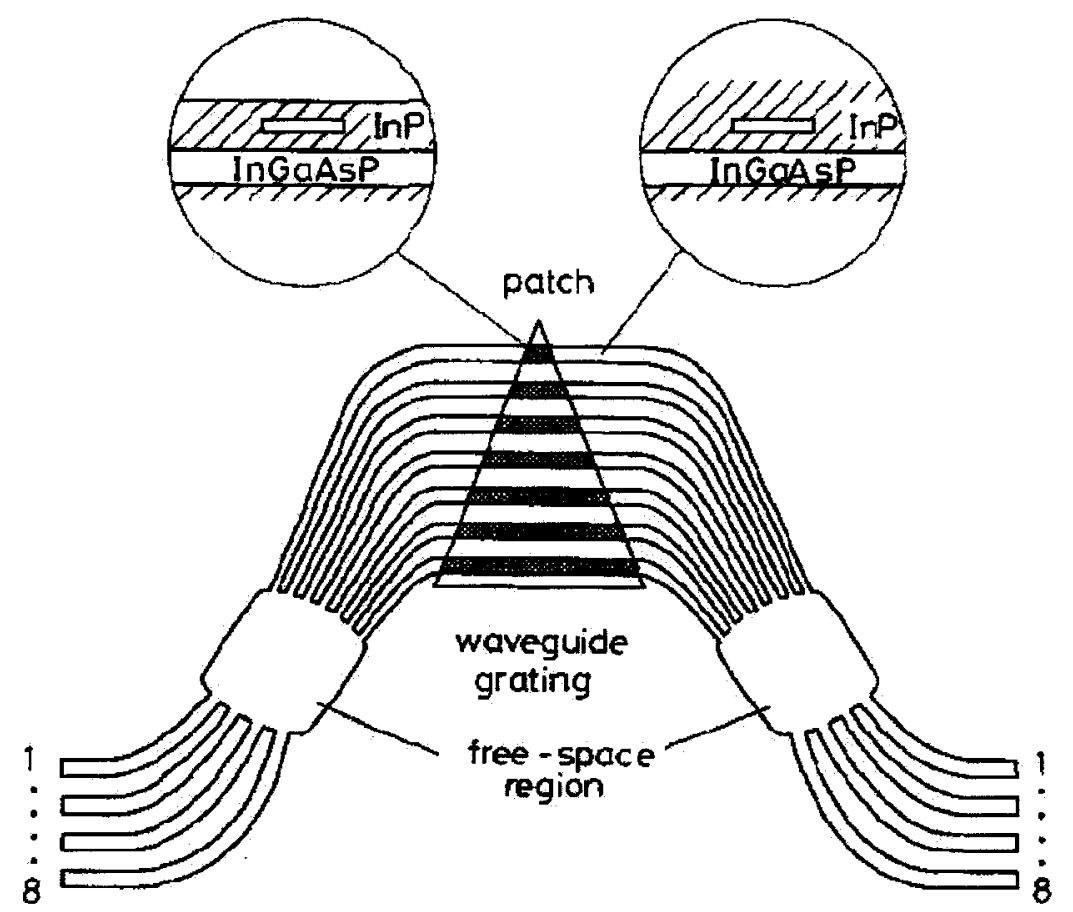

Figure 2.8: Schematic diagram of a polarization compensated waveguide grating router on InP. The InP cladding in the triangular patch section was $0.2 \mu \mathrm{m}$ thick while the cladding thickness was $1 \mu \mathrm{m}$ everywhere else. From [57].

etching the cladding layer of the slab waveguide. As mentioned earlier, this method was adopted in the silica-on-silicon demultiplexer design presented in Fig. 2.5.

\section{SOI-based Waveguide Devices}

SOI offers tremendous potential for cost-effective monolithic integration of optical and electronic devices on a same silicon substrate. The high-index contrast between the waveguide core and cladding permits strong light confinement and tight bending radius as small as a few microns. However, this high-index contrast property introduces large waveguide birefringence due to the electromagnetic boundary conditions imposed by the waveguide cross-section geometry. Therefore, most of the polarization control methods investigated for SOI waveguides have been focused on the waveguide cross-section modification to achieve polarization insensitivity.

In the case of SOI strip or photonic wire waveguides, single-mode operation is usual- 
ly obtained by designing waveguides with square cross-sections. An extreme compact dimension smaller than $0.35 \times 0.35 \mu \mathrm{m}^{2}$ is required for the single-mode propagation at the telecommunication wavelengths. The perfect symmetry in a waveguide with a square cross-section theoretically ensures the polarization independence in device performance due to the degeneracy between the TE and TM optical modes. Both theoretical and experimental work on the single-mode and polarization independent waveguide bends for optical interconnects have been reported $[59,60]$. Cassan et al. [59] presented a numerical analysis of a $90^{\circ}$-bend with a $4 \mu \mathrm{m}$ radius using SOI strip waveguides. The simulated

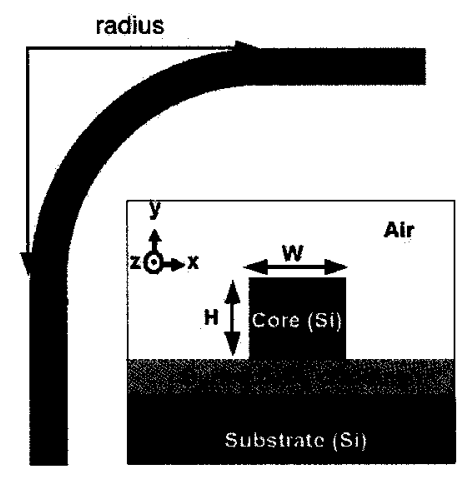

(a)

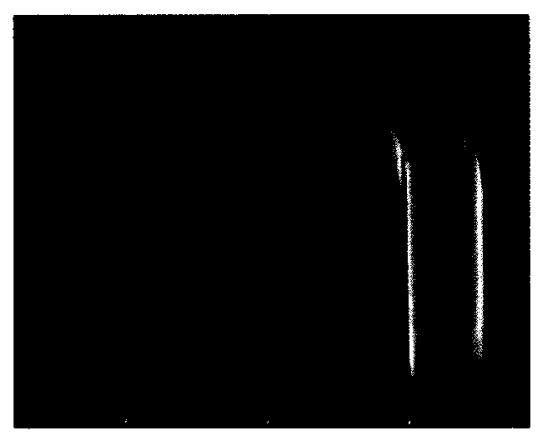

(b)

Figure 2.9: Polarization independent SOI waveguide bends: (a) schematic diagram of a waveguide $90^{\circ}$-bend structure with a radius of $4 \mu \mathrm{m}$ and the single-mode strip waveguide has a cross-section dimension of $0.35 \times 0.35 \mu \mathrm{m}^{2}$ (adapted from [59]); and (b) SEM image of a fabricated U-shaped $\left(180^{\circ}\right)$ bend with a radius of $2.75 \mu \mathrm{m}$ and the strip waveguide has a cross-section of $0.32 \times 0.45 \mu \mathrm{m}^{2}$ (adapted from [60]).

losses were below $0.1 \mathrm{~dB}$ for both polarizations and a square waveguide cross-section of $0.35 \times 0.35 \mu \mathrm{m}^{2}$ was used to ensure the polarization insensitivity of the bend structure. The designed device size was $16 \mu \mathrm{m}^{2}$.

Strip waveguides with rectangular cross-sections can also be single-mode. Baba et al. successfully fabricated a single-mode waveguide bend on SOI with a cross-section dimension of $0.32 \times 0.45 \mu \mathrm{m}^{2}$ [60], as illustrated in Fig. 2.9(b). With the given geometry of the waveguide cross-section, it was found that a U-shape $\left(180^{\circ}\right)$ bend structure effec- 
tively suppressed the polarization crosstalk. Based on such bend design, an ultracompact AWG was recently demonstrated [61], as shown in Fig. 2.10. The 9-channel device had

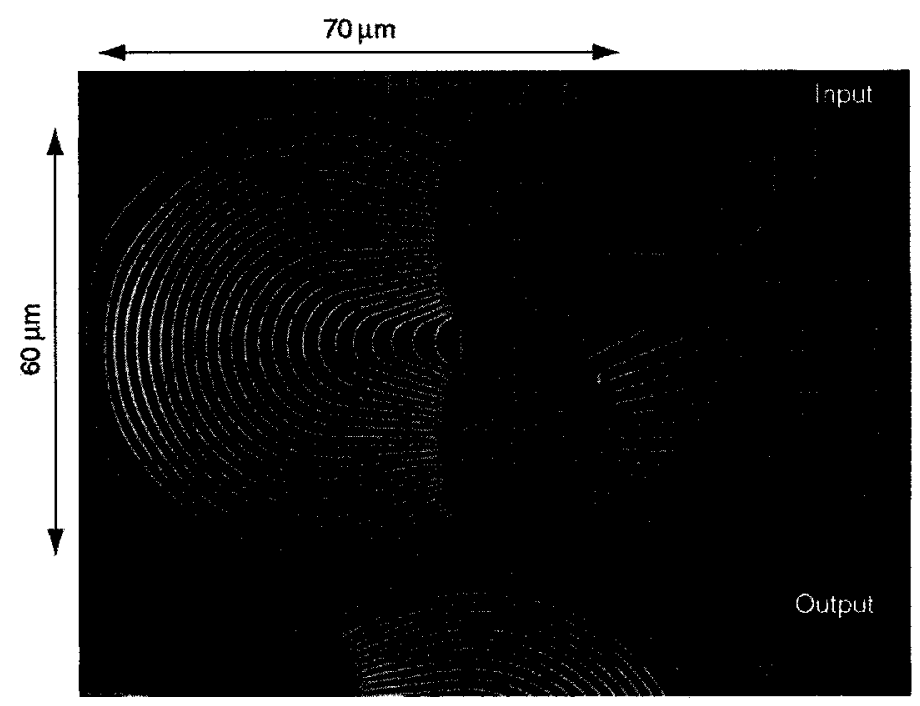

Figure 2.10: SEM image of the smallest reported AWG demultiplxer in SOI. The wire waveguides have a cross-section dimension of $0.32 \times 0.45 \mu \mathrm{m}^{2}$ and the overall device size is $\sim 70 \times 60 \mu \mathrm{m}^{2}$. From [61].

24 arrayed waveguides, with a total size of only $70 \times 60 \mu \mathrm{m}^{2}$, which is the smallest AWG device reported so far in literature. The measured channel spacing was $11 \mathrm{~nm}$, and the free spectral range (FSR) was $85 \mathrm{~nm}$. It should be pointed out this device is not polarization insensitive, only the TE polarization was used to demonstrate the demultiplexing function.

The design of a polarization insensitive AWG device based on silicon photonic wires were proposed in [62]. The wire was designed to have a cross-section of $0.297 \times 0.362 \mu \mathrm{m}^{2}$. The polarization dependent wavelength shift caused by the free-propagation region (FPR) was compensated by using different input waveguides for each polarization. The designed AWG device has 34 arrayed waveguides, with a device footprint of $316 \times 216 \mu \mathrm{m}^{2}$.

Ultracompact design of a polarization insensitive multimode interferometer (MMI) coupler based on silicon nanowire waveguides was also discussed by the same research group [63]. The MMI configuration is illustrated in Fig. 2.11. The polarization sensitivity of the MMI device was minimized by carefully choosing the length and width of the MMI 


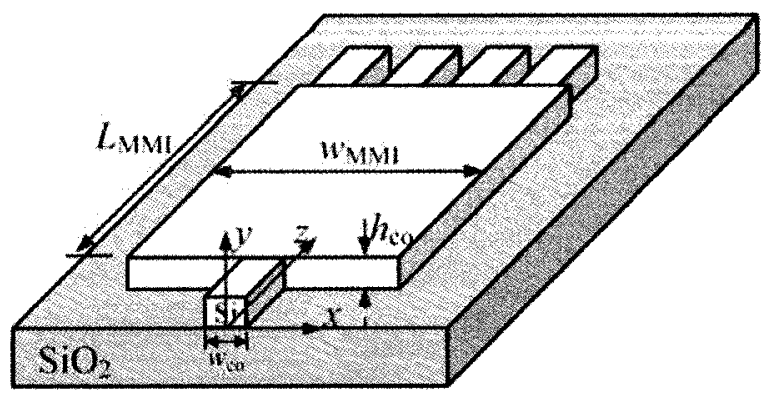

Figure 2.11: Schematic configuration of a polarization insensitive MMI coupler using SOI. The MMI has the following dimensions: $W_{\mathrm{MMI}}=$ $3.86 \mu \mathrm{m}, L_{\mathrm{MMI}}=15.3 \mu \mathrm{m}$. The wire waveguides have square cross-sections with $W_{\text {co }}=H_{\text {co }}=0.4 \mu \mathrm{m}$. From [63].

section. Simulations showed that an MMI with $W_{\mathrm{MMI}}=3.86 \mu \mathrm{m}, L_{\mathrm{MMI}}=15.3 \mu \mathrm{m}$, and a thickness of $H_{\mathrm{co}}=400 \mu \mathrm{m}$ could provide a polarization insensitive splitting function.

In the case of SOI ridge waveguides, the polarization sensitivity and the single-mode characteristics as a function of geometrical parameters have been studied extensively [27, $30,64]$. The single-mode ridge waveguides are typically not birefringence-free $[21,26]$. However, Vivien et al. showed that in principle it may be possible to achieve zero birefringence while maintaining single-mode propagation by an appropriate choice of the ridge geometrical parameters [30]. Fig. 2.12(a) and (b) illustrate the simulated waveguide geometries (including ridge height, width and etch depth) that satisfy single-mode and birefringence-free conditions simultaneously at the operating wavelength of $1530 \mathrm{~nm}$ and $1550 \mathrm{~nm}$, respectively $[27,30]$.

The geometrical modification is the general approach for most of the reported SOI ridge waveguide devices in obtaining polarization independent operation. For example, Trinh et al. demonstrated a polarization insensitive AWG device by tailoring the waveguide geometry [65]. The silicon core has a ridge height of $5 \mu \mathrm{m}$ with an etch depth of $3 \mu \mathrm{m}$. A TE-TM wavelength shift of $0.02 \mathrm{~nm}$ was observed at the operating wavelength of $1550 \mathrm{~nm}$. The 4-channel AWG device with 30 arrayed waveguides have a relatively large size of $2.7 \times 2.7 \mathrm{~cm}^{2}$, due to the use of large bending radii $(2 \mathrm{~cm})$ for the arrayed waveguides. 


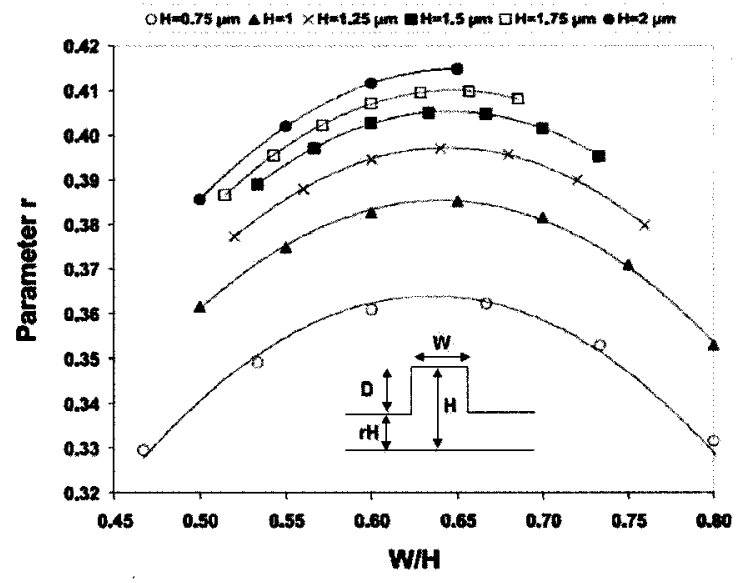

(a)

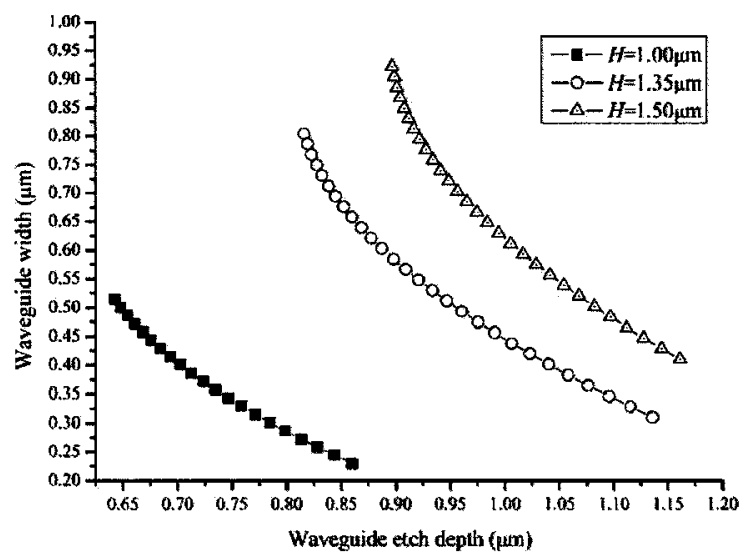

(b)

Figure 2.12: Waveguide geometries that satisfy both single-mode and birefringence-free condition simultaneous for various waveguide ridge heights: (a) at $\lambda_{0}=1530 \mathrm{~nm}$ (From Ref. [30]), and (b) at $\lambda_{0}=1550 \mathrm{~nm}$ (From Ref. [27]).

Cheben et al. presented two compact polarization independent AWG demultiplexers $[7,66]$. Both AWG devices have 100 arrayed waveguides with a $200 \mathrm{GHz}$ channel spacing, as illustrated in Fig. 2.13(a). The total chip size for both cases is $\sim 5 \times 5 \mathrm{~mm}^{2}$. The ridge waveguides were fabricated on much thinner silicon layers than that in Ref. [65], with $H_{\mathrm{Si}}=1.5$ and $2.2 \mu \mathrm{m}$, respectively. Although a polarization compensator on the slab free-propagation region (FPR) was introduced to both AWG demultiplexers, the polarization compensation mechanism was different for each device. The cross-section of 


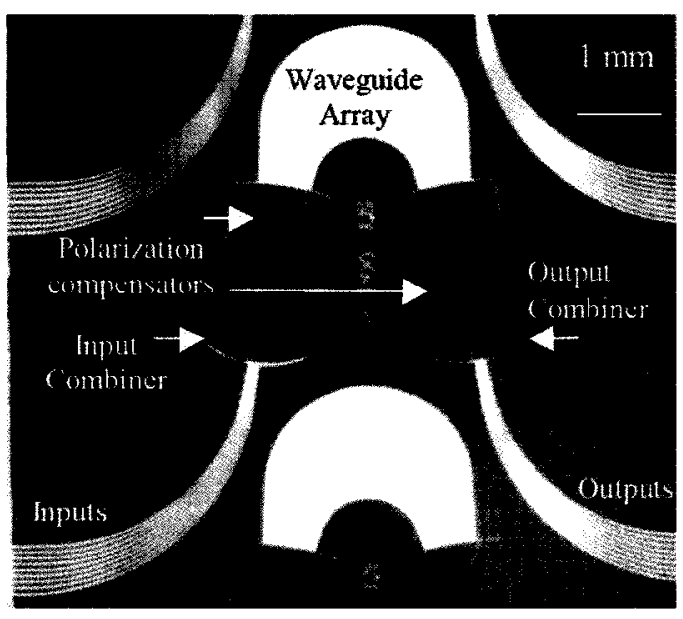

(a)
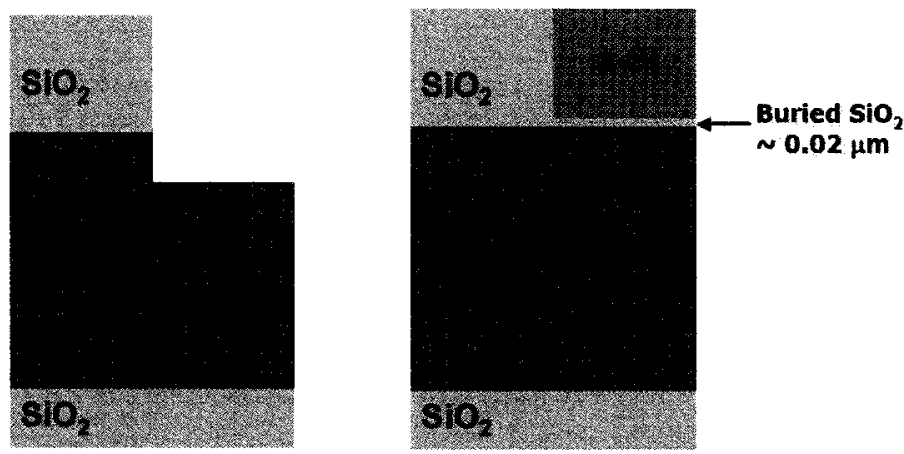

(b)

Figure 2.13: (a) Microscope image of an SOI AWG demultiplexer with two identical polarization compensators in the slab FPR regions (adapted from $[7,66])$; (b) cross-section of the polarization compensation region: an etched compensator for the AWG device in Ref. [7] (left) and a silicon-oxidesilicon (SOS) compensator for the device in Ref. [66] (right).

the FPR regions of the two devices are shown in Fig. 2.13(b). In the device presented in Ref. [7], the polarization compensation was achieved by etching a prism-like region in the slab. The optical path lengths of the TE and TM modes in the slab regions are effectively altered, such that the relative phase differences between TE and TM can be compensated and completely eliminated. Fig. 2.13(b)[left] shows that all of the cladding and parts of the core layer must be etched away for a full birefringence elimination. The mode-mismatch at the boundary between the compensator and unmodified slab 
waveguide is expected to cause significant optical loss. To improve the mode-mismatch, the deposition of a silicon cap layer of a-Si or polysilicon was proposed, as depicted in Fig. 2.13(b)[right]. Such silicon-oxide-silicon (SOS) structure was predicted to alter the birefringence of the slab waveguide, with negligible change to the waveguide mode at the compensator boundary. The two birefringence compensation methods had already been successfully applied for the InP-and the silica-on-silicon based diffraction grating spectrometers $[33,46]$, respectively, which were discussed earlier in the chapter.

An optical ring resonator is a versatile structure that has been developed to create many different optical devices, including filters, switches, and add/drop multiplexers in dense WDM systems and sensors in biomedical appliacations. The extremely compact size of the micro-resonators is made possible by the use of strongly confined waveguides using high-refractive-index-contrast material systems (e.g., SOI), which allow the resonators to have very small radii without significant bend loss. Most of the SOI ring resonators have been realized by using photonic wire waveguides, with the bend radii on the order of microns $[6,9,10]$. However, these ultracompact microrings are usually polarization dependent due to the different propagation losses and coupling efficiencies experienced by the TE and TM modes. In many cases, only one polarization (i.e., either TE or TM) is chosen for demonstrating the switching, filtering, and add/drop functions.

Recently, Headley et al. [67] demonstrated polarization independent ring resonator using ridge waveguides, as illustrated in Fig. 2.14. To achieve a fully polarization independent operation, it is required that the waveguides are birefringence-free, while the directional coupler between the ring and the bus waveguide has equal coupling coefficients for the TE and TM polarizations. The cross-section geometry of the waveguide was carefully tailored to satisfy the single-mode and birefringence-free conditions: $H=1.34 \mu \mathrm{m}$, $W=0.87 \mu \mathrm{m}$, and $D=0.67 \mu \mathrm{m}$. The directional coupler between the $400 \mu \mathrm{m}$-radius ring and the bus waveguide has a coupling separation of $0.65 \mu \mathrm{m}$. Although this design yielded a polarization independent compact resonator with a free spectral range of 


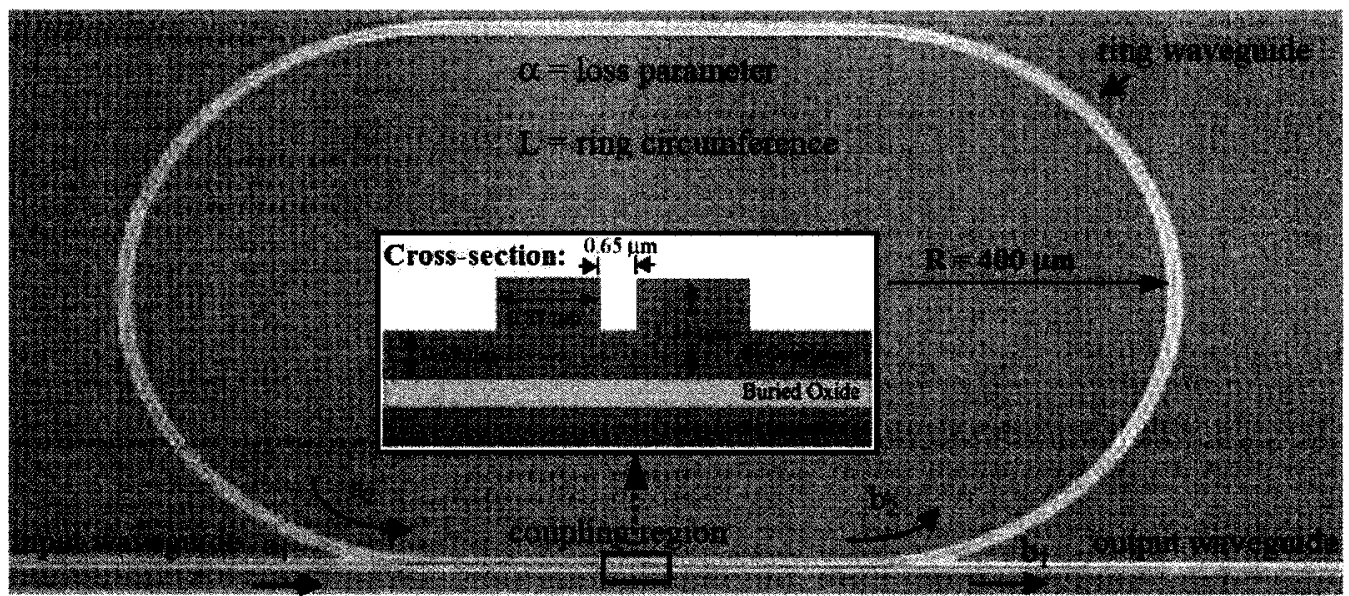

Figure 2.14: Microscope image of a compact polarization independent racetrack ring resonator on SOI. The cross-section of the directional coupler is shown in the inset. From [67].

$F S R=193 \mathrm{~nm}$, a reasonable finesse of 12 , and a high Q-factor of 90000 , the device performance is extremely sensitive to the coupling length and separation, waveguide geometry, and bending radius of the ring. The fabrication of both the ridge waveguides and the direction coupler is extremely challenging as the geometry has very narrow dimensional tolerances.

It is clear from the above examples that it is possible to minimize waveguide birefringence by adjusting the waveguide cross-section geometry. However, this method becomes increasingly difficult for waveguides with small cross-sections as the core thickness reduces to well below the wavelength of light $[27,30,64]$. The use of the cladding stress for polarization control in SOI-based waveguides was first proposed by S. W. Roberts at Bookham Technology in a patent filed in 2001 [68]. The author found that the stress induced by the thermal oxide cladding layer could change the birefringence in SOI ridge waveguides. Polarization independent operation was achieved in an SOI AWG device by a patterned thin thermal oxide patch in the grating section, as shown in Fig. 2.15. However, the relationship between the stress induced birefringence and the design parameters of the AWG ridge waveguides was not discussed.

The first investigation of the polarization characteristics of SOI waveguides with 


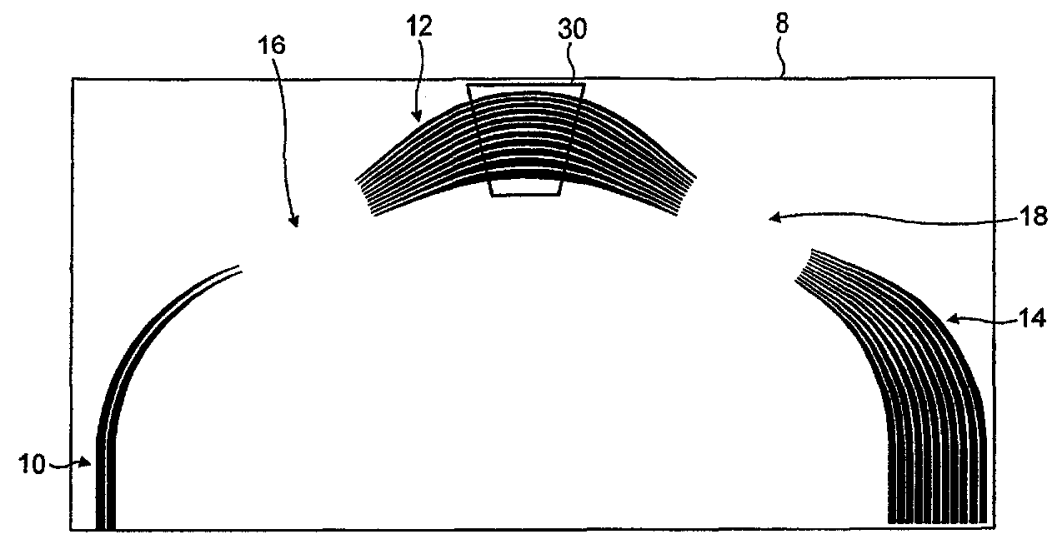

Figure 2.15: A schematic plan view of an SOI AWG device. The ridge waveguides has the following dimensions: width $W=5.8 \mu \mathrm{m}, H=4.3 \mu \mathrm{m}$, $D=1.7 \mu \mathrm{m}$. The truncated triangular region 30 is the patterned thermal oxide patch. From [68].

square and ridge cross-sections under stress were presented by the Optoelectronic group at National Research Council in 2004 [69], and the details will be presented in Chapter 3 and 4 . The same group recently successfully demonstrated a polarization insensitive ring resonator using an MMI coupler and stress-induced birefringence [70,71]. Fig. 2.16 shows the design parameters of the ridge waveguide, the ring resonator itself, and the MMI coupler. The MMI couplers were designed to produce identical splitting ratios

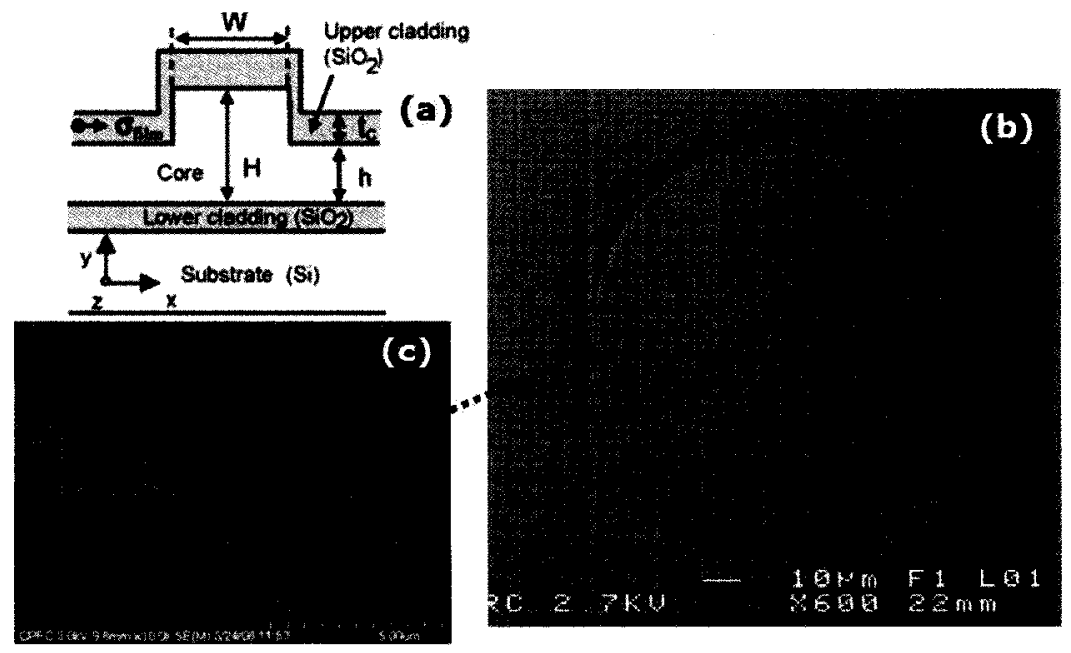

Figure 2.16: (a) Cross-section of the SOI ridge waveguide: $W=H=$ $1.5 \mu \mathrm{m}, H-h=0.9 \mu \mathrm{m}$; (b) a ring resonator using an MMI coupler with a polarization independent MMI coupler: $R=200 \mu \mathrm{m}$; (c) a close-up of the MMI coupler: $W_{\mathrm{MMI}}=6 \mu \mathrm{m}$ and $L_{\mathrm{MMI}}=54 \mu \mathrm{m}$. From $[70,71]$ 
for both polarizations. Preliminary measurements showed that an upper oxide cladding thickness of $0.8 \mu \mathrm{m}$ with a $-250 \mathrm{MPa}$ compressive film stress completely compensated the geometrical birefringence from the waveguides [71].

\subsubsection{Polarization Splitting}

For many birefringent optical devices, a polarization diversity strategy is a straightforward solution, where the two orthogonally polarized TE and TM components of the optical signal are separated and processed individually. In fact, polarization splitting and filtering are fundamental operations in optical signal processing and monitoring, with applications ranging from network monitoring, polarization mode dispersion compensation, to polarimetric sensors. Waveguide polarization splitters, filters, and converters are the key components in the polarization diversity approach [72]. Different configurations of polarization splitters and filters such as the Y-branch, multimode interference coupler, directional coupler, Mach-Zehnder interferometer, Bragg gratings, photonic crystal, arrayed waveguide gratings have been reported. These devices have been realized on silica-on-silicon, $\mathrm{LiNbO}_{3}$, III-V semiconductor, and SOI.

\section{Silica Waveguide Devices}

Vellekoop et al. [73] suggested a polarization splitter based on an AWG configuration. The operating principles of an AWG will be discussed in more detail in Chapter 7. Fig. 2.17 shows the device details of their AWG-based polarization splitter using an $\mathrm{SiO}_{2} / \mathrm{Al}_{2} \mathrm{O}_{3} / \mathrm{SiO}_{2}$ waveguide structure on a silicon substrate. The device consists of an input waveguide and a number of output waveguides, both coupled to a waveguide array through two slab FPR regions. Due to the linearity in the length increment in the arrayed section, the phase change of different wavelength channels varies linearly along the output plane; that is,

$$
\Delta \phi=\frac{2 \pi}{\lambda_{0}} n_{\mathrm{eff}} \Delta L
$$




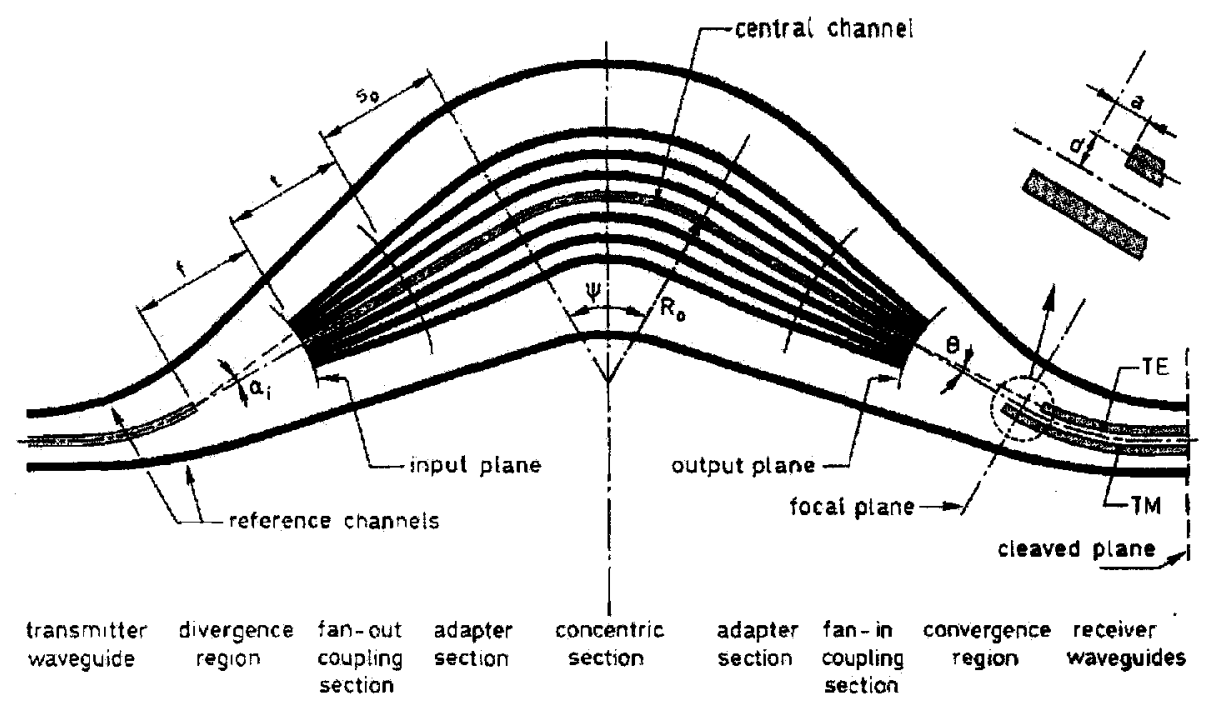

Figure 2.17: Polarization splitter geometry with waveguide phased array configuration based on an $\mathrm{SiO}_{2} / \mathrm{Al}_{2} \mathrm{O}_{3} / \mathrm{SiO}_{2}$ waveguide structure on a silicon substrate. From [73].

where $\lambda_{0}$ is the operating wavelength, $n_{\text {eff }}$ is the polarization dependent effective index, and $\Delta L$ is the constant length increment in the arrayed section. This phase shift in turn determines the angle $\theta$ of the output beam, and hence the position of the focused beam at the focal plane. The channel length increment $\Delta L$ is carefully chosen such that the total length of each channel is equal to an integer multiple of the operating wavelength. Since the phase transfer is determined by the product of the effective index of TE or TM and the total length of each channel, a small variation in the polarization dependent index will result in a variation in the phase transfer, thus leading to a lateral shift of the focal point at the receiver side. By placing the output waveguides at proper positions along the image plane on the receiver side, spatial separation of the TE and TM polarizations can be obtained. This splitter was demonstrated with a low insertion loss of $0.5 \mathrm{~dB}$ and a polarization cross-talk of $17-20 \mathrm{~dB}$ at the operating wavelength of $633 \mathrm{~nm}$. This device has a narrow operating bandwidth since the splitting of the two polarizations is determined by the wavelength dependent phase shift. The overall chip size is $0.6 \times 2.5 \mathrm{~mm}^{2}$

Okuno et al. [42] reported a polarization splitter based on a Mach-Zehnder interferom- 
eter (MZI) configuration (see Fig. 2.18). The birefringence modification using a stressed a-Si film has been discussed for the devices in Refs. [43], [33] and [66]. In this MZI-based polarization splitter device, two stressed a-Si films were equipped on the waveguide arms. One was a $90 \mu \mathrm{m}$ wide a-Si film on the top MZI arm for controlling the phase state without changing the waveguide birefringence, while the other was a $50 \mu \mathrm{m}$ wide a-Si film on the bottom MZI arm for controlling the waveguide birefringence. The waveguide birefringence and the phase states of the device were accurately controlled by laser trimming of the a-Si films. The output polarization states were switched by controlling the thermo-optic phase shifter. A polarization crosstalk of $25.6 \mathrm{~dB}$ were obtained at 1557 $\mathrm{nm}$.

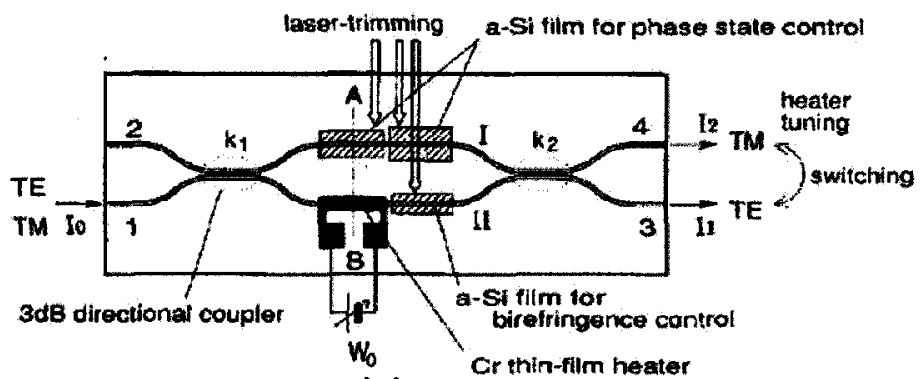

(a)

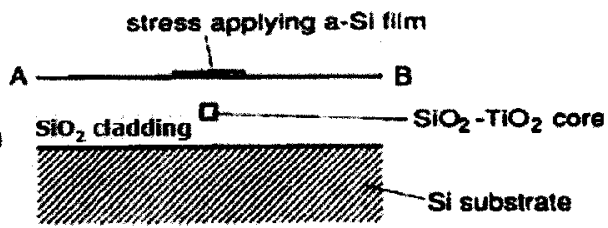

(b)

Figure 2.18: (a) Configuration of a MZI-based polarization splitter using a stressed a-Si film; (b) the cross-section geometry of the upper MZI arm: the core had a dimension of $8 \times 8 \mu \mathrm{m}^{2}$, and the a-Si film was $7 \mu \mathrm{m}$ thick and had a width ranged from 50 to $100 \mu \mathrm{m}$. From [42].

\section{$\mathrm{LiNbO}_{3}$ And III-V Semiconductor Devices}

Most earlier work on polarization splitters has relied on the electro-optic effect, where the refractive index of a medium can be controlled by externally applied electric field. This effect is frequently used for phase modulation in non-centrosymmetric crystals such as $\mathrm{LiNbO}_{3}[74]$ and III-V compound semiconductors [75]. In the polarization splitter device using $\mathrm{LiNbO}_{3}$ waveguides presented in Ref. [74], a directional coupler configuration was used. An external voltage of $20 \mathrm{~V}$ was supplied to the electrodes on the waveguides to achieve the necessary phase shift in achieving polarization splitting at $1150 \mathrm{~nm}$. An 
electro-optic TE/TM mode splitter using InGaAsP-InP waveguides was demonstrated in a Mach-Zehnder interferometer (MZI) configuration (Fig. 2.19). The light is divided into

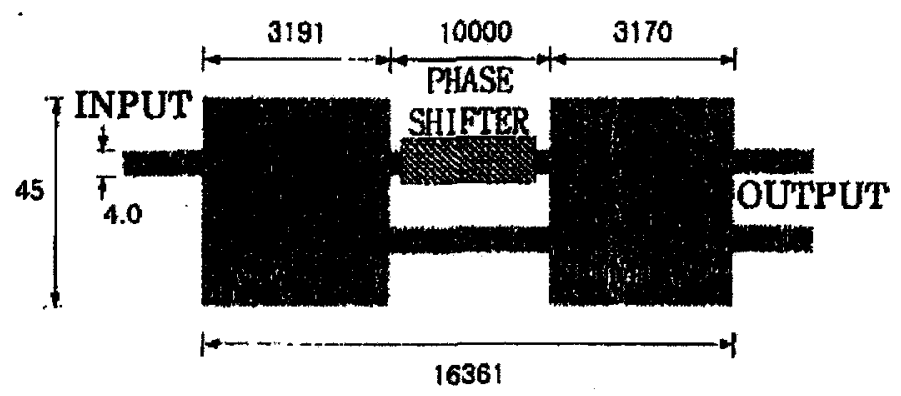

Figure 2.19: Schematic diagrams of an electro-optic polarization splitter with an MZI configuration. Waveguides were fabricated using InGaAsP-InP. From [75].

two interferometer arms using a $3 \mathrm{~dB}$-splitter. In this example, multimode interference (MMI) couplers were used as the $3 \mathrm{~dB}$ couplers. The polarization dependent phase shifts between the light beams traveling in the two arms determine the constructive or destructive interference at the output $3 \mathrm{~dB}$ coupler. For a complete splitting or filtering effect, the induced phase shift between the TE and TM polarization must be $\pi$. The device in Fig. 2.19 is based on the principle that the phase of the TE mode can be altered by the electro-optic effect in undoped III-V Schottky-barrier ridge waveguides while the TM mode remains almost unchanged with relatively small applied electric field [75]. With a reverse bias voltage of $5 \mathrm{~V}$, a polarization splitting function was observed at $1550 \mathrm{~nm}$. Both mentioned $\mathrm{LiNbO}_{3}$ and $\mathrm{InP}$ based devices are large in size, with a device length larger than $16 \mathrm{~mm}$.

Polarization splitters have also been reported using an asymmetric Y-branch configuration in $\mathrm{LiNbO}_{3}$ [76-78]. Fig. 2.20 shows that this type of splitters which consists of three sections: the input section, a straight output branch, and a bent output branch. The basic idea behind the polarization splitting is that the two output arms of the Ybranch have different material index for the TE and TM modes, such that the waveguides are in the cut-off regime for the unwanted polarization and only the desired polarization 


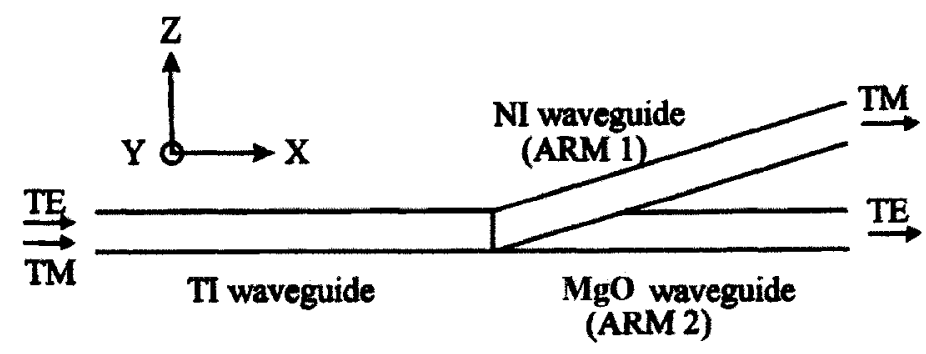

Figure 2.20: Schematic diagram of a $1 \times 2$ asymmetric Y-branch polarization splitter. From [76].

is guided in a given branch. In this example, the Ti diffused $\mathrm{LiNbO}_{3}$ waveguide is allowed to guide both polarizations. In contrast, under a well defined diffusion condition of $\mathrm{Ni}$, a waveguide can only guide the TE polarized light; while the TM polarization propagates along the $\mathrm{MgO}$ diffused waveguide [76]. In other words, a polarization splitter was fabricated where the TE and TM modes were originally guided by a Ti-diffused input waveguide and then directed to the $\mathrm{MgO}$ - and $\mathrm{Ni}$-diffused waveguides according to the orientation of $\mathrm{LiNbO}_{3}$ substrate. The measured extinction ratios were greater than 20 $\mathrm{dB}$ for both TE and TM modes. Although $\mathrm{LiNbO}_{3}$ is widely used commercially, high external voltages, long device lengths, and complicated diffusion processes are the main drawbacks.

A polarization compensator using a stressed cladding film has been adopted to make III-V semiconductor based polarization splitters. Both MZI [79] and directional coupler [80] configurations using this technique were demonstrated. Fig. 2.21 shows an example of the MZI-based InP device with two MMI 3dB couplers. At least one waveguide arm needs to have modified birefringence in order to achieve sufficient polarization dependent phase shifts such that the light interferes constructively or destructively at the output $3 \mathrm{~dB}$ coupler. In this example, the birefringence in the top interferometer arm is enhanced by depositing a strained titanium metal film to induce a $\pi$ phase difference between the TE and TM polarization. The splitting extinction ratio was obtained to be better than $-13 \mathrm{~dB}$ for both polarizations over a $60 \mathrm{~nm}$ wavelength range, with the best extinction ratio of $-19 \mathrm{~dB}$ measured at $1510 \mathrm{~nm}$. 


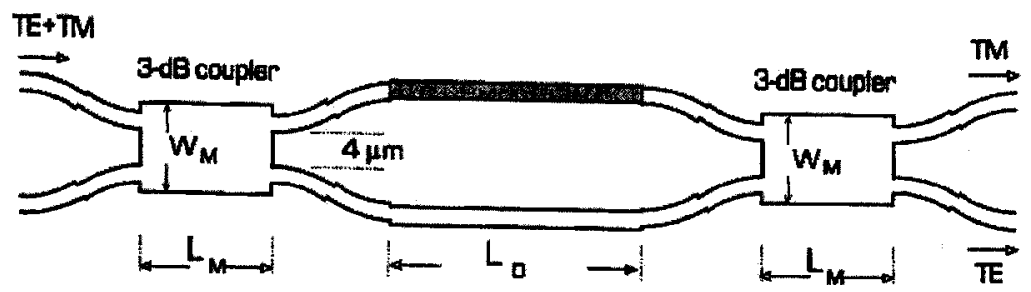

Figure 2.21: Schematic of an MZI-based polarization splitter, with two MMI $3 \mathrm{~dB}$ couplers. The shaded area indicates the Ti metal- $\mathrm{SiO}_{2}$ cladding on the top of the InP waveguide. From [79].

\section{SOI-based Waveguide Devices}

All the SOI-based polarization splitter devices reported in literature have focused on the waveguide cross-section modification. In the example of a directional coupler based polarization splitter, as illustrated in Fig. 2.22a, the device has two output ports in which an input is coupled into the cross-state waveguide for one polarization (Output 3) and in the bar state waveguide for the other (Output 2). The principle of operation can

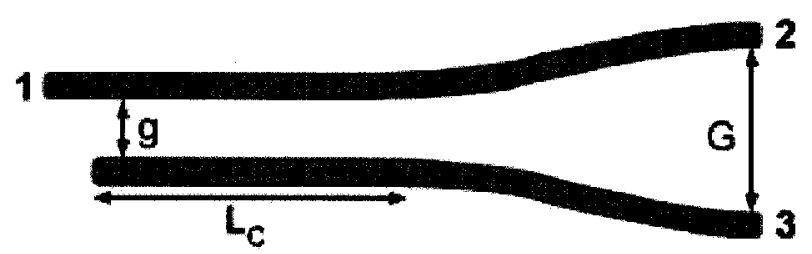

(a)

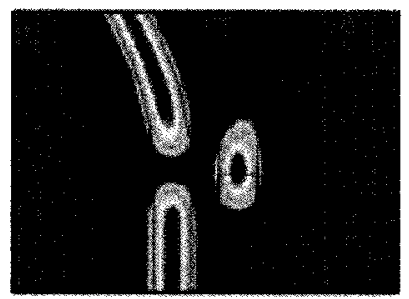

(b)

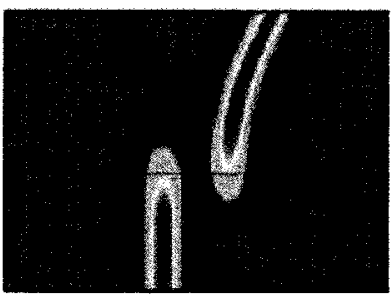

(c)

Figure 2.22: Schematic of a directional coupler based polarization splitter. (a) Top view of the device layout. At a chosen coupling length, $L_{c}$, the mode profiles show that (b) the TE mode stays in bar state; (c) the TM mode transfers to the cross-state. From [81].

be described as follows: the input light that propagates in a directional coupler can be expressed in terms of even and odd modes of the coupled waveguide system with effective 
indices $n_{e}$ and $n_{o}$. The coupling length $L_{c}$ is defined as the length necessary for complete energy transfer for either TE or TM polarization, and is given by:

$$
L_{c}=\frac{\pi}{\left(n_{e}-n_{o}\right) k_{0}}
$$

where $k_{0}$ is the free space wave number. Since $n_{e}$ and $n_{o}$ are polarization dependent, the coupling length $L_{c}$ is strongly polarization dependent. For a full polarization splitting, the following condition must be satisfied:

$$
\frac{L_{c}^{\mathrm{TM}}}{L_{c}^{\mathrm{TE}}}=2 M
$$

where $M$ is an integer. This is the fundamental condition that governs the directional coupler based polarization splitters.

In silica-based systems, the difference between the coupling coefficients for TE and $\mathrm{TM}$ is very small, therefore a sufficiently large coupling length is normally required in a functional splitter [82]. Much shorter devices can be designed in the high-index contrast SOI platform. Recently, Kiyat et al. reported the first integrated polarization splitter in SOI waveguides (Fig. 2.22) [81]. This simple and compact SOI device has a length of $120 \mu \mathrm{m}$ and a coupling spacing of $0.7 \mu \mathrm{m}$. However, precise control of the coupling length and spacing are necessary for the full splitting of TE and TM polarizations, as illustrated in Fig. 2.22(b-c). The silicon ridge height and width used in this work were both $1 \mu \mathrm{m}$, while the etch depth was chosen to be $0.4 \mu \mathrm{m}$. Polarization splitting was observed at $1550 \mathrm{~nm}$ with extinction ratios of $18.1 \mathrm{~dB}$ for the TM polarization and 8.0 $\mathrm{dB}$ for the TE. The main drawback of this device is the low fabrication tolerance because the device performance is extremely sensitive to the spacing between the two waveguides in the coupling region. The directional coupler based devices in general suffer a narrow operating wavelength range (polarization splitting is observed at only $1550 \mathrm{~nm}$ ) and stringent fabrication tolerances.

Liang et al. [12] demonstrated a $3 \mathrm{~mm}$ long broadband MZI-based polarization splitter. The silicon core had a nominal thickness of $5 \mu \mathrm{m}$, with an etch depth of $2.5 \mu \mathrm{m}$. 
Fig. 2.23 illustrates the device configuration. The ridge waveguide widths of the two Mach-Zehnder arms are $2 \mu \mathrm{m}$ and $3.5 \mu \mathrm{m}$, respectively, which introduce a relative phase difference between the TE and TM polarization. The splitting condition is satisfied when a $\pi$ difference is achieved from the geometrical birefringence induced by the ridge width variation. The device showed a splitting extinction ratio of at least $-12 \mathrm{~dB}$ across wavelengths ranging from $1515 \mathrm{~nm}$ to $1565 \mathrm{~nm}$.

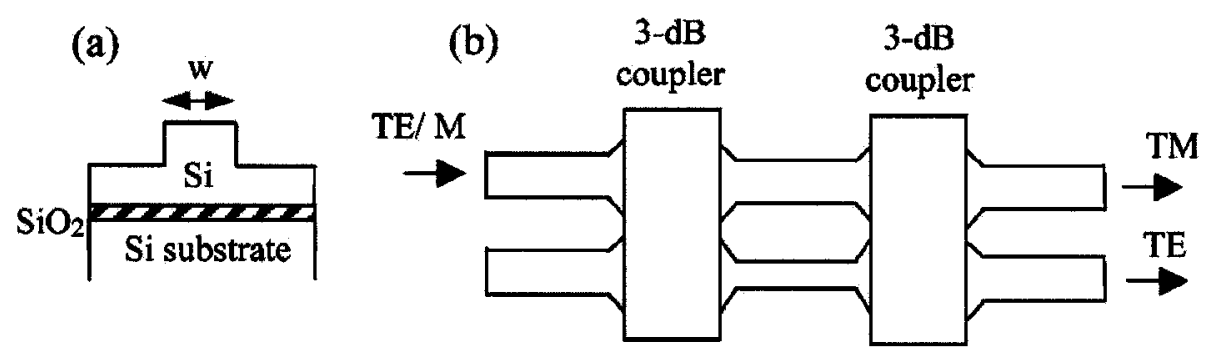

Figure 2.23: (a) Cross-section of the SOI ridge waveguide; (b) schematic layout of the MZI based polarization splitter. From [12].

\subsection{Thesis Objective}

It is clear from the literature that polarization analysis, control, and manipulation are vital in the design and the operation of optical devices. Past research has explored a variety of methods for polarization management in planar waveguide systems based on silica-on-silicon, III-V semiconductors and SOI. However, limitations in fabrication tolerances, constraints in the geometrical design for optimal performance, and feasibility in implementation of the method still remain the toughest obstacles to the development of viable microphotonic polarization control devices. In addition, most of the polarization control methods are specific to the polarization dependence of the silica-based waveguides. For SOI based devices, the method of tailoring the waveguide cross-section geometry is widely used to achieve the polarization splitting function. These reported devices are associated with specific dimension requirements and may have very low fabrication tolerances. Moreover, geometrical modification may introduce undesirable compromises 
in the overall system performance [72] because the cross-sectional geometry affects other design criteria such as mode confinement, bend loss, minimum allowed bend radius, and coupling between adjacent waveguides. Low-cost, compact, reliable, practical, and integration compatible polarization control devices are yet to be developed. The ultimate goal of the thesis project is to identify, investigate, and implement an effective and practical method for polarization management in the high-index contrast SOI material system, and apply this method to novel and compact polarization control devices. The project includes the following components:

1. To explore and implement an effective method of polarization management for SOI waveguides;

2. To experimentally demonstrate the feasibility of the proposed approach;

3. To model and design novel polarization control devices which utilize the proposed method for polarization manipulation;

4. To fabricate the polarization control devices and characterize the device performance. 


\section{Chapter 3}

\section{Stress: Theory and Modeling}

The previous chapter reviewed recent advances in polarization management. Most methods were developed for birefringence control and elimination in the weakly guided systems, primarily the silica-based waveguides. For high-index contrast material systems such as SOI, the technique of tailoring waveguide cross-section aspect ratio has been widely used for polarization management $[2,30]$.

Fig. 3.1 shows the geometrical birefringence $\left(\Delta n_{\text {geo }}=n_{\text {eff }}^{\mathrm{TM}}-n_{\text {eff }}^{\mathrm{TE}}\right)$ for a single-mode ridge waveguide, whose geometrical profile satisfies the single-mode condition. It confirms that the geometrical birefringence, arising from different boundary conditions of the E-field polarized in parallel and perpendicular direction to an interface, can be zero for single-mode waveguides with a given geometrical profile. However, as the waveguide core size scales down to $2 \mu \mathrm{m}$ and below, the magnitude of the birefringence increases exponentially and becomes very sensitive to the variations in the waveguide dimensions. This finding can be generalized for waveguides of arbitrary cross-section. Fig. 3.1 shows that it may be impossible to maintain both single mode behavior and polarization independence in waveguides simultaneously with small core dimensions. We can conclude that although this approach can be useful in controlling birefringence $[12,81]$, it is not practical for microphotonic devices with small core dimensions due to the stringent fab- 


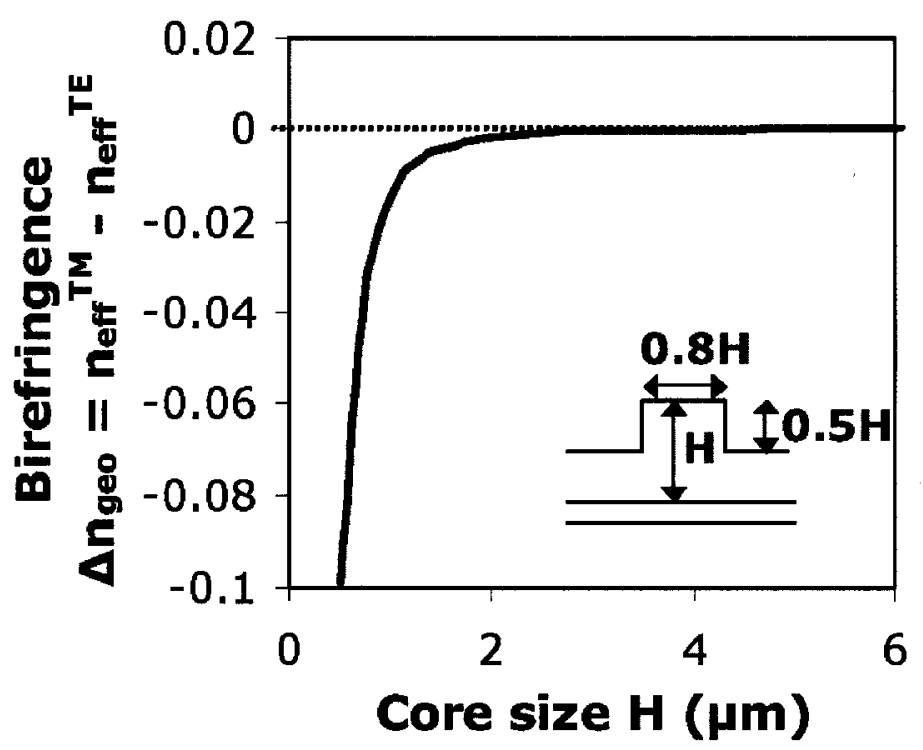

Figure 3.1: The influence of core dimensions on waveguide geometrical birefringence $\left(\Delta n_{\text {geo }}\right)$ for a geometry shown in the inset.

rication requirements on waveguide geometry. In addition, by adopting this approach, the waveguide core geometry is fixed by the birefringence constraints. It may be difficult to optimize device performance and accommodate other design criteria such as mode confinement, bend loss, minimum allowed bend radius, and coupling between adjacent waveguides. To overcome the challenges, limitations and constraints faced in this traditional approach, other means of polarization management in SOI system need to be explored.

In silica-based waveguide devices, stress caused by the mismatch between the silicon substrate and the silica waveguide core is the primary concern for the polarization independent performance in devices such as wavelength (de)multiplexers. As described in Chapter 2, stress compensation provides an elegant means for waveguide birefringence tuning, and is an extremely important topic in silica-based waveguide technology. However, the impact of stress in high-index contrast systems such as SOI has not been generally recognized [15].

This chapter introduces the concept of stress engineering in SOI by reviewing the photoelastic relations in waveguides. Then it investigates the simulation modeling of the 
stress-induced effects in waveguides. Two commonly used analytical models for stress calculations are compared: the generalized plane-strain method and the ordinary planestrain approximation. A simple and accurate calculation scheme, called the "Normalized Plane-Strain Model", is then proposed, derived and implemented. This newly developed numerical model will be used in the investigation of the stress-induced effects for the rest of the research project.

\subsection{Waveguide Stress and Strain: Theory}

Let us first review the basic concepts in stress engineering. The overall stress in a film consists of two types: the intrinsic and the thermal stress. The intrinsic stress arises from the physical properties of the material such as the lattice mismatch between adjacent layers, and is generated during the material growth process. The thermal stress results from the mismatch in the thermal expansion coefficients of the film and the substrate, and the temperature change between the deposition and the final room temperatures. Despite the different origins of the two stress types, they are indistinguishable in the experimental stress measurements. The intrinsic stress in SOI is expected to be negligible because the silicon substrate and the silicon core obviously have identical lattice constants and thermal expansion coefficients. Thus in waveguide simulations we normally use the thermal stress to represent the overall stress experienced in a waveguide system. In a typical SOI ridge waveguide as shown in the inset of Fig. 3.2, the stress in the $\mathrm{SiO}_{2}$ upper cladding film produces a stress distribution within and near the embedded Si ridge, which in turn causes a change in the refractive index in both the $\mathrm{Si}$ and $\mathrm{SiO}_{2}$ layers via the photoelastic effect. The stress-induced change in material refractive index can be described by the following relations $[22,83]$ :

$$
\begin{gathered}
\Delta n_{x}=n_{x}-n_{0}=-C_{1} \sigma_{x}-C_{2}\left(\sigma_{y}+\sigma_{z}\right), \\
\Delta n_{y}=n_{y}-n_{0}=-C_{1} \sigma_{y}-C_{2}\left(\sigma_{x}+\sigma_{z}\right),
\end{gathered}
$$




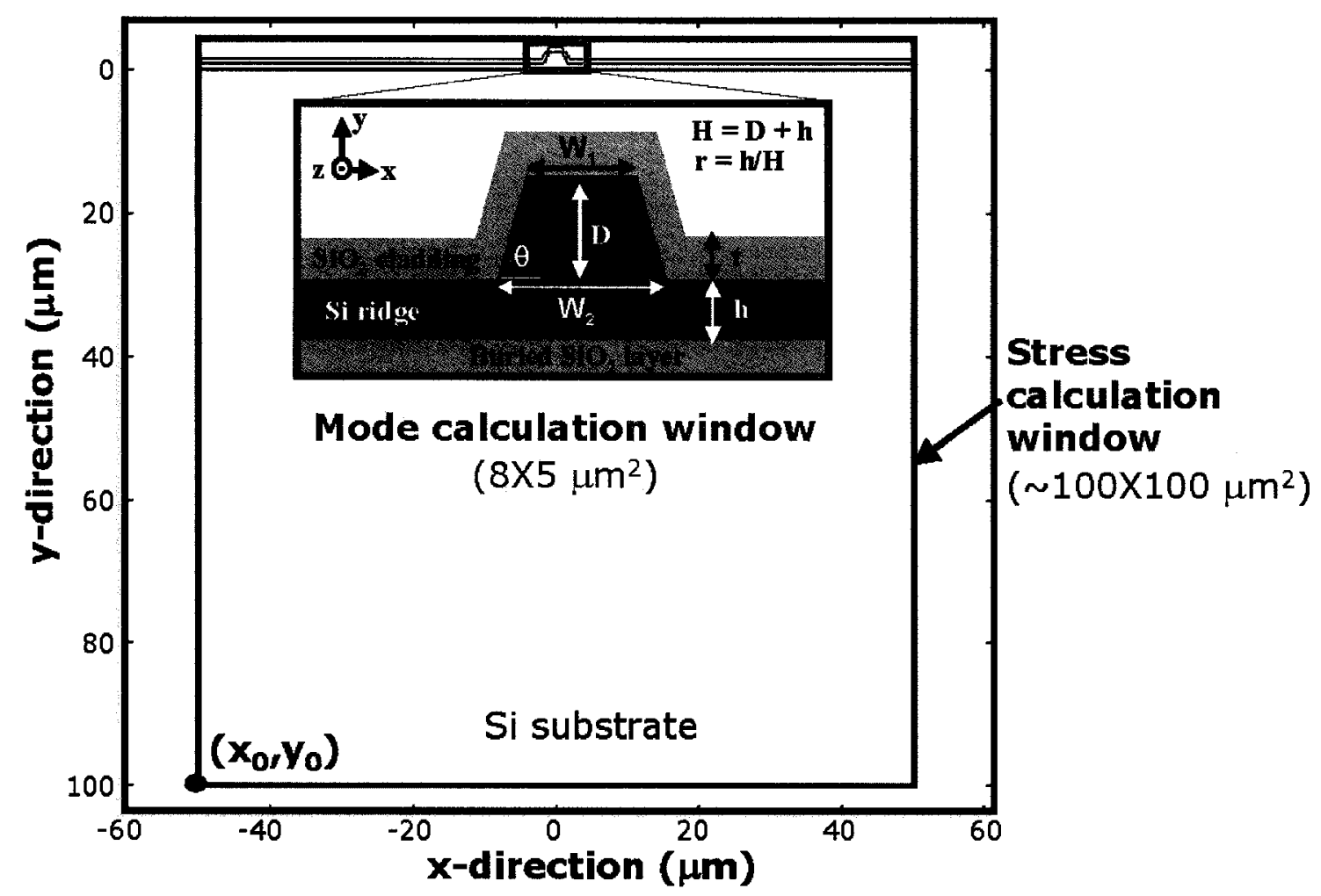

Figure 3.2: Typical simulation calculation window: a $100 \times 100 \mu \mathrm{m}^{2}$ window for stress analysis and a $8 \times 5 \mu \mathrm{m}^{2}$ window for modal analysis. Inset: a close-up view of a typical ridge waveguide in the mode calculation window.

where $\sigma_{x}, \sigma_{y}$, and $\sigma_{z}$ are the principal stress tensor components along the $x-, y$-, and $z$-axis directions, respectively; $n_{0}$ is the refractive index of the material without stress; and $C_{1}$ and $C_{2}$ are the stress-optical constants, related to the Young's modulus $(E)$, Poisson's ratio $(\nu)$, and the stress-optic tensor elements $\left(p_{11}\right.$ and $\left.p_{12}\right)[22,83]$ as:

$$
\begin{gathered}
C_{1}=\frac{n^{3}}{2 E}\left(p_{11}-2 \nu p_{12}\right), \\
C_{2}=\frac{n^{3}}{2 E}\left[-\nu p_{11}+(1-\nu) p_{12}\right] .
\end{gathered}
$$

Both the material refractive index and the stress-optic tensor elements depend on the wavelength, hence the stress-optical constants $C_{1}$ and $C_{2}$ are also wavelength dependent. In this work, the operating wavelength is assumed to be $\lambda=1550 \mathrm{~nm}$. Table 3.1 summarizes the material parameters used in our simulations, which are quoted from Landolt-Bornstein [84]. Since the stress-optic tensor elements $\left(p_{i j}\right)$ were measured at 
$1150 \mathrm{~nm}$, the stress-optical constants derived from them may not be completely accurate at $1550 \mathrm{~nm}$. However, the dispersion in $p_{i j}$ is expected to be weak since the strong electronic transitions occur at much shorter wavelengths for both $\mathrm{Si}$ and $\mathrm{SiO}_{2}$.

\begin{tabular}{|c|c|c|c|c|c|c|}
\hline \multirow[b]{2}{*}{ Material } & \multirow{2}{*}{$\begin{array}{l}\text { Young } \mathrm{s} \text { modulus, } \\
E \text { (CPa) }\end{array}$} & \multirow{2}{*}{$\begin{array}{l}\text { Poisson's } \\
\text { ratio, } \nu\end{array}$} & \multirow{2}{*}{$\begin{array}{l}\text { Thermal expatsion } \\
\text { coefficient, } \alpha\left(\mathrm{K}^{-1}\right)\end{array}$} & \multirow{2}{*}{$\begin{array}{c}\text { Refractive index } \\
n\end{array}$} & \multicolumn{2}{|c|}{ Photoelastic tensor elements ${ }^{b}$} \\
\hline & & & & & $p_{11}$ & $p_{12}$ \\
\hline $\mathrm{Si}$ & 130 & 0.27 & $3.6 \times 10^{-6}$ & 3.476 & -0.101 & 0.0094 \\
\hline $\mathrm{SiO}_{2}$ & 76.7 & 0.186 & $5.40 \times 10^{-7}$ & 1.444 & 0.16 & 0.27 \\
\hline
\end{tabular}

Table 3.1: Material parameters of Si (substrate and waveguide core) and $\mathrm{SiO}_{2}$ (upper and lower claddings).

The modeled SOI waveguide system, shown in Fig. 3.2, consists of the silicon substrate, the buried $\mathrm{SiO}_{2}$ layer, the $\mathrm{Si}$ waveguide layer including the etched ridge waveguide, and an overlying $\mathrm{SiO}_{2}$ cladding. The structure is assumed to be invariant along the waveguide propagation direction. The modeled system was set to be $\sim 100 \times 100 \mu \mathrm{m}^{2}-$ large enough that edge effects do not distort the stress distribution in the vicinity of the ridge waveguide. The boundary conditions are chosen such that the physical system is "locked" at the lower left corner $\left(x_{0}, y_{0}\right)$, while the top, bottom and sides of the modeled system are surfaces that can move freely in $x$ - and $y$-directions response to the internal stresses. In our calculations, we assume that all the material layers are at equilibrium at a reference temperature $\left(T_{\text {ref }}\right)$, which corresponds to the deposition temperature for an idealized oxide film on a silicon system. As a result, there is no intrinsic stress in any layer at the reference temperature.

The strain and stress components, $\epsilon_{i}$ and $\sigma_{j}$, are related as follows, given the difference between the operating and reference temperatures $\left(\Delta T=T_{0}-T_{\text {ref }}\right)$ :

$$
\left[\begin{array}{c}
\varepsilon_{x} \\
\varepsilon_{y} \\
\varepsilon_{z}
\end{array}\right]=\frac{1}{E}\left(\left[\begin{array}{ccc}
1 & -\nu & -\nu \\
-\nu & 1 & -\nu \\
-\nu & -\nu & 1
\end{array}\right]\left[\begin{array}{c}
\sigma_{x} \\
\sigma_{y} \\
\sigma_{z}
\end{array}\right]\right)+\alpha \Delta T
$$

where $\alpha$ is the material thermal expansion coefficient, $\varepsilon_{x}, \varepsilon_{y}$, and $\varepsilon_{z}$ correspond to the 
principal strain along the $x, y$, and $z$ directions, respectively. According to Eq. 3.3, the strain consists of two parts: the elastic strain (first term) and the thermal-induced strain (second term). By inverting Eq. 3.3, an expression for the stress distribution tensors $\left(\sigma_{i}\right)$ can be obtained as in Eq. (3.4).

$$
\left[\begin{array}{c}
\sigma_{x} \\
\sigma_{y} \\
\sigma_{z}
\end{array}\right]=\frac{E}{(1+\nu)(1-2 \nu)}\left[\begin{array}{ccc}
1-\nu & \nu & \nu \\
\nu & 1-\nu & \nu \\
\nu & \nu & 1-\nu
\end{array}\right]\left[\begin{array}{c}
\varepsilon_{x} \\
\varepsilon_{y} \\
\varepsilon_{z}
\end{array}\right]-\frac{\alpha E \Delta T}{1-2 \nu}
$$

Since the thermal expansion coefficient of silicon is approximately seven times larger than that of $\mathrm{SiO}_{2}$ (see Table 3.1), a compressive stress is developed in an oxide layer as the deposited oxide film cools down to the room temperature on a silicon wafer. Normally, the in-plane stress component is significantly larger than the out-plane component; that is, $\left|\sigma_{x}\right| \gg\left|\sigma_{y}\right|$. We use a film stress parameter $\sigma_{\text {film }}$ to quantify and characterize the average in-plane stress $\sigma_{x}$ created in a uniform two-dimensional oxide film after cooling. The magnitude of the stress level $\sigma_{\text {film }}$ depends on the intrinsic material properties of the deposited film and the substrate, such as their thermal expansion coefficients and compositions, as well as the deposition condition and subsequent annealing. In this work, $\sigma_{\text {film }}$ is determined from a location far away from the ridge in numerical simulations. The corresponding value of the film stress $\sigma_{\text {film }}$ in experimental devices can be obtained from the bow radius of a wafer coated with a stressed film. For example, stress in a PECVD grown $\mathrm{SiO}_{2}$ film typically ranges from $\sigma_{\text {film }}=-100$ to $-400 \mathrm{MPa}$. In comparisons of theoretical results with experiments, the experimentally measured $\sigma_{\mathrm{film}}$ is used as the target stress level in the simulation analysis by varying $\Delta T$ in the modeling system. 


\subsection{Stress Modeling}

\subsubsection{Numerical Simulation Tool}

Chapter 1.2.3 described the finite element method (FEM) which can be used for solving Maxwell's equation for the TE- and TM-like waveguide mode distributions and the

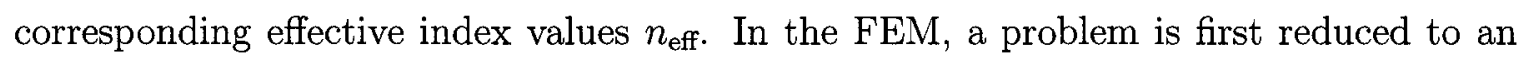
eigenvalue equation. Then the physical system is divided into a large number of discrete triangular units or mesh elements. The division of the elements depends on the natural geometrical boundaries and the material properties of the domain. The overall solution of the entire domain is based on the eigen solutions to each individual mesh element. The stress field in the waveguide, expressed as in Eq. 3.4, can also be numerically solved by using the FEM.

A commercial software program, FEMLAB ${ }^{\circledR}$, has been used to solve the stress distributions in the waveguide. Stress analysis in FEMLAB ${ }^{\circledR}$ is based on the principle of virtual work, which states that the sum of the virtual work from the internal strains is equal to the work from external loads. A static equilibrium equation is expressed and solved by $\mathrm{FEMLAB}^{\circledR}$ for the stress distribution which simultaneously satisfies the stress-strain relation, the thermal effects, and the strain-displacement relation, for the displacement variables in the $x-, y$-, and $z$-direction, under proper boundary conditions. Once the stress distribution in the vicinity of the ridge waveguide is known, the local refractive index distribution can be evaluated using Eq. 3.1.

In order to evaluate the stress-induced change in the effective indices of TE- and TM-like modes, the stress-induced material refractive index change will be included in the wave equation. Similar to the mode analysis described in Chapter 1.2.3, the wave equation and the boundary conditions for each mode (TE- or TM-like mode) are first transformed into a differential equation. Then a variational method is applied to the differential equation, and the solution to each mesh element is evaluated and combined 
to form an overall solution over the entire analysis domain. The effective index $\left(n_{\mathrm{eff}}\right)$ for each mode under stress can therefore be determined.

Since the mesh density (i.e., the number of elements) is crucial to the accuracy of the overall solution, convergence analyses have been performed to establish the minimum number of mesh elements. The rule of thumb is that a high mesh density should be set for regions with abrupt changes in the material index profile. In the simulations carried out in the project, the number of mesh elements is usually over 38,000 for a computation window size of $100 \mu \mathrm{m} \times 100 \mu \mathrm{m}$ (as in Fig. 3.2), with a maximum element area of around $600 \mathrm{~nm}^{2}$. In the next three sections, different plane-strain calculation schemes will be described. The evaluation and comparison of the models are all performed using FEMLAB ${ }^{\circledR}$.

\subsubsection{Generalized Plane Strain Model}

A generalized plane strain model assumes that the strain along the direction of waveguide propagation (i.e., the $z$-direction) is independent of $z$ but can vary with $x$ and $y$. This model holds for waveguides that are invariant along the propagation direction and are much longer than their cross-section dimensions. In this formulation, the wafer is allowed to deform in response to film stress in the $x$-, $y$ - or $z$-direction. The strain in the $z$ direction, in the simplest approximation, is constrained to vary linearly in the $x-y$ plane. That is,

$$
\varepsilon_{z}=\varepsilon_{0}+a x+b y
$$

where $\varepsilon_{0}, a$ and $b$ are constants. $\varepsilon_{0}$ depends mainly on the material's thermal expansion coefficient $(\alpha)$ and the temperature change $(\Delta T)$. The value of $b$ is usually much larger than that of $a$ since wafer bending requires that the in-plane strain varies in the $y$ direction across the wafer thickness, while bending should be independent of the in-plane coordinates, $x$ and $z$, far away from the wafer edges.

Stress distributions in an SOI ridge waveguide, calculated from the generalized plane 
strain model using the FEM are shown in Fig. 3.3. The $x, y$, and $z$ components of the anisotropic stress distribution in the ridge and its cladding layer change the material refractive index in the waveguide, as described in Eq. 3.1. The positive and negative values of the stress represent the tensile and compressive forces, respectively. The film stress far away from the ridge is found to be $\sigma_{\text {film }}=\sigma_{x} \approx-100 \mathrm{MPa}$, based on a temperature change of $\Delta T=380^{\circ} \mathrm{C}$ and a film thickness of $1 \mu \mathrm{m}$. To derive an expression for the in-plane stress $\sigma_{\text {film }}$ in the $\mathrm{SiO}_{2}$ cladding, we substitute the strain approximation in the $z$-direction (Eq. 3.5) into the general stress-strain relation described in Eq. 3.4, and obtain:

$$
\sigma_{\mathrm{film}}=\sigma_{x(\mathrm{avg})}=\left(\alpha_{s}-\alpha\right) \frac{E}{1-\nu} \Delta T
$$

where $\alpha_{s}$ and $\alpha$ are the thermal expansion coefficients of the Si substrate and the cladding, respectively. Note that the above equation calculates the stress level in a uniform film on a thick substrate.

As shown in Fig. 3.3, a compressive cladding stress along the $x$-direction induces an anisotropic stress in the silicon ridge waveguide: a compressive stress in the $x$-direction and a tensile stress in the $y$-direction. The influence of stress on waveguide birefringence will be further discussed later in Chapter 4 .

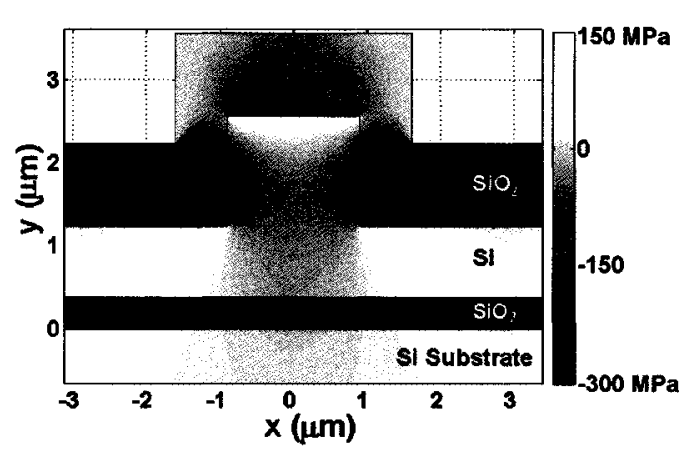

(a)

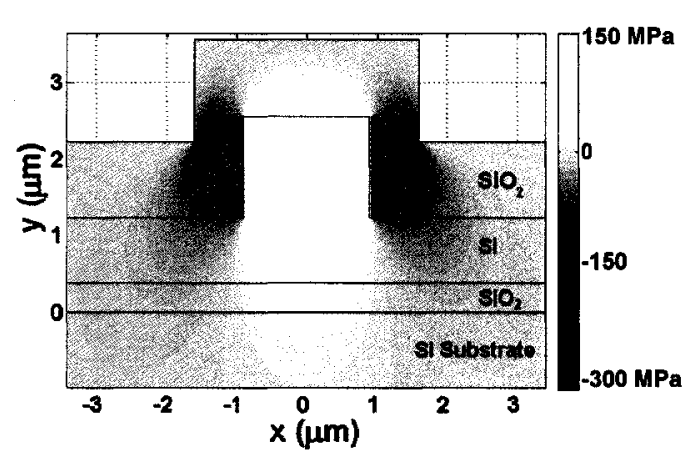

(b)

Figure 3.3: The $x$ and $y$-components of the stress distributions in an SOI ridge waveguide: (a) the stress field $\sigma_{x}$ and (b) the stress field $\sigma_{y}$. The buried oxide layer thickness is $0.37 \mu \mathrm{m}$, the upper oxide cladding thickness is $1 \mu \mathrm{m}$, and Si ridge height and width are $2.2 \mu \mathrm{m}$ and $1.8 \mu \mathrm{m}$, respectively. 


\subsubsection{Ordinary Plane Strain Model}

One of the main drawbacks associated with using the fully generalized plane strain model is that for a system large enough to avoid edge effects, but with a fine enough mesh to accurately calculate the stress fields, the calculation takes a long time and requires a large computation memory. This model can be simplified by assuming that the strain component along the propagation $z$ direction is negligible compared to strains in $x$ and $y$ directions [85]. Under this plane strain assumption, the model system is not allowed to either expand or contract in $z$ direction (i.e., $\varepsilon_{z}=0$ ). The three-dimensional stress analysis (Eq. 3.4) is reduced to the equations:

$$
\begin{gathered}
\sigma_{x}=\frac{E}{(1+\nu)(1-2 \nu)}\left[(1-\nu) \epsilon_{x}+\nu \epsilon_{y}\right]-\frac{\alpha E \Delta T}{1-2 \nu} \\
\sigma_{y}=\frac{E}{(1+\nu)(1-2 \nu)}\left[\nu \epsilon_{x}+(1-\nu) \epsilon_{y}\right]-\frac{\alpha E \Delta T}{1-2 \nu} \\
\sigma_{z}=\nu\left(\sigma_{x}+\sigma_{y}\right)-\alpha E \Delta T .
\end{gathered}
$$

Here the equations for $\sigma_{x}$ and $\sigma_{y}$ are decoupled from $\sigma_{z}$, resulting in a simpler twodimensional problem for the stress distribution in the $x-y$ plane. Although this model has been widely used to calculate stress in waveguides ( $[22,42,86,87])$, the condition that $\epsilon_{z}=0$ introduces an artificial tensile stress $\sigma_{z}$ into the waveguide Si core and the substrate, and reduces the compressive stress in the cladding layers along the $z$-axis. These changes in stress cause a uniform shift in the calculated material refractive indices, $n_{x}$ and $n_{y}$, which in turn change the effective indices of the waveguide modes. This can be important in modeling interference based devices when stress is present.

The artificial stress levels in the $\mathrm{Si}$ and the $\mathrm{SiO}_{2}$ layers along $z$ also change their relative thermal expansion in the $x-y$ plane. For example, in the case of a uniform $\mathrm{SiO}_{2}$ film on a thick Si substrate, by allowing unconstrained thermal expansion in all directions in the generalized plane strain model, the in-plane stress $\sigma_{\text {film }}$ in the $\mathrm{SiO}_{2}$ cladding is given by Eq. 3.6. On the other hand, with a suppressed strain component $\varepsilon_{z}=0$ in the ordinary plane strain model described by Eq. 3.7, the effective in-plane stress along the $x$-axis is 
therefore:

$$
\sigma_{\text {film }}=\left(\frac{1+\nu_{s}}{1+\nu} \alpha_{s}-\alpha\right) \frac{E}{1-\nu} \Delta T
$$

where the parameters with a subscript $s$ represent the properties of the Si substrate. Evaluating the expressions Eqs. 3.6 and 3.8, the ordinary plane strain model overestimates the film stress $\sigma_{\text {film }}$ by approximately $10 \%$, and therefore the stress-induced birefringence by a similar amount.

\subsubsection{Normalized Plane Strain Model}

In this section, a modified form of the ordinary plane strain model that correctly reproduces the stress component along the propagation $z$-axis is proposed. This simple normalized plane strain model achieves comparable accuracy as the fully generalized plane strain model presented in section 3.2.2. If we neglect wafer bending along the $z$-axis (the bending effects will be discussed later in this section) and assume that the top layers conform to the substrate during cooling, the strain component in the $z$ direction takes the form of $\varepsilon_{z}=\alpha_{s} \Delta T$, without the $x$ and $y$ dependency in Eq. 3.5. This approximation assumes the waveguide displacement in the $z$-direction is determined by the thermal expansion of the substrate. This plane strain model yields the stress-strain relation as follows:

$$
\begin{gathered}
\sigma_{x}^{\prime}=\frac{E}{(1+\nu)(1-2 \nu)}\left[(1-\nu) \epsilon_{x}+\nu \epsilon_{y}\right]-\left(\alpha-\frac{\nu}{1+\nu} \alpha_{s}\right) \frac{E \Delta T}{1-2 \nu} \\
\sigma_{y}^{\prime}=\frac{E}{(1+\nu)(1-2 \nu)}\left[\nu \epsilon_{x}+(1-\nu) \epsilon_{y}\right]-\left(\alpha-\frac{\nu}{1+\nu} \alpha_{s}\right) \frac{E \Delta T}{1-2 \nu} \\
\sigma_{z}^{\prime}=\nu\left(\sigma_{x}^{\prime}+\sigma_{y}^{\prime}\right)-\left(\alpha-\alpha_{s}\right) E \Delta T .
\end{gathered}
$$

Comparing Eq. 3.9 to Eq. 3.7, the equations for $\sigma_{x}^{\prime}$ and $\sigma_{y}^{\prime}$ are again decoupled from $\sigma_{z}^{\prime}$. Furthermore, the normalized plane strain equations (Eq. 3.9) for $\sigma_{x}^{\prime}$ and $\sigma_{y}^{\prime}$ are identical to the ordinary plane strain equations for the stress fields $\sigma_{x}$ and $\sigma_{y}$ as in Eq. 3.7, if a normalized thermal expansion coefficient in each layer, $\alpha^{\prime}$, is applied:

$$
\alpha^{\prime}=\alpha-\frac{\nu}{1+\nu} \alpha_{s}
$$


Solving an ordinary plane strain problem using Eq. 3.7 with $\alpha^{\prime}$ will shift the value for $\sigma_{z}$ in each layer by a constant amount. However, since the resulting difference in $\sigma_{z}$ is just a uniform constant in each layer, the correct value for $\sigma_{z}^{\prime}$ is obtained by adding a constant correction term to $\sigma_{z}$ :

$$
\sigma_{z}^{\prime}=\sigma_{z}+\frac{E}{1+\nu} \alpha_{s} \Delta T
$$

where the stress field $\sigma_{z}$ is calculated as in Eq. 3.7, with the inclusion of Eq. 3.10 as the thermal expansion coefficients. This means that the same algorithm used for the ordinary plane strain model can be employed to evaluate the $x$ and $y$-components of the stress distribution in the waveguides, with simple substitutions of the normalized thermal expansion coefficients. 


\begin{tabular}{|c|c|c|c|c|c|c|}
\hline Etch Depth & Model & $\begin{array}{c}\sigma_{x} \\
(\mathrm{MPa})\end{array}$ & $\begin{array}{c}\sigma_{y} \\
(\mathrm{MPa})\end{array}$ & $\begin{array}{c}\sigma_{z} \\
(\mathrm{MPa})\end{array}$ & $\begin{array}{c}\text { Birefringence } \\
\Delta n_{\mathrm{eff}}\end{array}$ & $\begin{array}{c}\% \text { change } \\
\text { in } \Delta n\end{array}$ \\
\hline \hline \multirow{2}{*}{$D=1.2 \mu \mathrm{m}$} & Generalized Plane Strain (GPS) Model & -269 & 0.02 & -273 & 0.002334 & - \\
& Ordinary Plane Strain (OPS) Model & -291 & 0.02 & -13.5 & 0.002597 & $(+11.27 \%)$ \\
& Normalized Plane Strain (NPS) Model & -269 & 0.02 & -280 & 0.002327 & $(-0.31 \%)$ \\
\hline \multirow{2}{*}{$D=1.34 \mathrm{~m}$} & GPS Model & -269 & 0.02 & -273 & 0.003651 & - \\
& OPS Model & -291 & 0.02 & -13.5 & 0.003960 & $(+8.47 \%)$ \\
& NPS Model & -268 & 0.02 & -280 & 0.003643 & $(-0.22 \%)$ \\
\hline \multirow{2}{*}{$D=1.5 \mu \mathrm{m}$} & GPS Model & -269 & 0.02 & -273 & 0.004903 & - \\
& OPS Model & -291 & 0.02 & -13.4 & 0.005254 & $(+7.16 \%)$ \\
& NPS Model & -268 & 0.02 & -280 & 0.004894 & $(-0.18 \%)$ \\
\hline
\end{tabular}

Table 3.2: Comparison of oxide film stress $\sigma_{\text {film }}$ and waveguide birefringence calculations from generalized, ordinary, and normalized plane strain models. The stress values are taken at the middle of the oxide film, $4 \mu \mathrm{m}$ away from the center of the ridge waveguide. The SOI waveguide has the following dimensions: $H=2.2 \mu \mathrm{m}, W=1.8 \mu \mathrm{m}$, and $t=1 \mu \mathrm{m}$. The stress calculation window is $100 \mu \mathrm{m}$ ( wide) $\times 100 \mu \mathrm{m}$ thick (see Fig. 3.2). Material parameters listed in Table 3.1 were used for these calculations. 
Table 3.2 compares the waveguide birefringence and the stress $\sigma_{\text {film }}$ in a $1 \mu \mathrm{m}$-thick upper cladding film obtained from the different plane strain models using numerical analysis. The simulated ridge waveguide has a core height of $2.2 \mu \mathrm{m}$ and width of $1.8 \mu \mathrm{m}$. The lower oxide cladding has a thickness of $0.37 \mu \mathrm{m}$. The results are compared for different ridge etch depths $D$, assuming a difference between the operating and reference temperature of $\Delta T=-980^{\circ} \mathrm{C}$. Using the generalized plane strain model as the benchmark, it is shown that the ordinary plane strain model overestimates the birefringence by about $10 \%$ for all the three cases, as expected from Eqs. 3.7 and 3.8. When modeling birefringence in interferometric devices such as arrayed waveguide gratings (AWGs), this birefringence error would produce a wavelength shift ranging from $\Delta \lambda=0.07$ to 0.15 $\mathrm{nm}$, which represents up to $20 \%$ of the WDM channel spacing in an $100 \mathrm{GHz}$ (or 0.8 $\mathrm{nm}$ ) spaced AWG device. The normalized model, on the other hand, produces virtually identical stress levels and birefringence as the fully generalized model, yet requires an order of magnitude less computation time. This is to our knowledge, the first time this extension of the ordinary plane strain model has been presented for stress calculations in optical waveguides.

\subsubsection{Stress Relief and Calculation Window Size}

Stress relief is an important factor in determining the total stress in finite systems, and must be included when comparing the theoretical results with the experimental measurements. When internal stresses are present, both real and modeled systems will deform to reduce stress. In the SOI waveguide system stress relief will occur through bending of the wafer, and through elastic deformations extending inwards from the edges of the wafer and the ridge itself. The magnitude and range of these effects must be understood to correctly choose the calculation window size of the modeled system, and to assess measurements of film stress and waveguide birefringence on finite substrates.

For a slab SOI system, assuming a substrate with a thickness $d$ much larger than the 
film thickness $t$ (i.e., $d \gg t$ as illustrated in Fig. 3.4), the stress relief in the $\mathrm{SiO}_{2}$ cladding due to the wafer bending is given by [88]:

$$
\frac{\Delta \sigma}{\sigma_{0}}=-\frac{4 t E\left(1-\nu_{s}\right)}{d E_{s}(1-\nu)}
$$

where $\Delta \sigma$ is the change in stress associated with bending, and $\sigma_{0}$ is the stress in the cladding film with no substrate bending. Although this equation is derived for a single layer on a substrate, it is approximately correct for our SOI system provided the film thickness includes both the buried oxide and oxide cladding thickness.

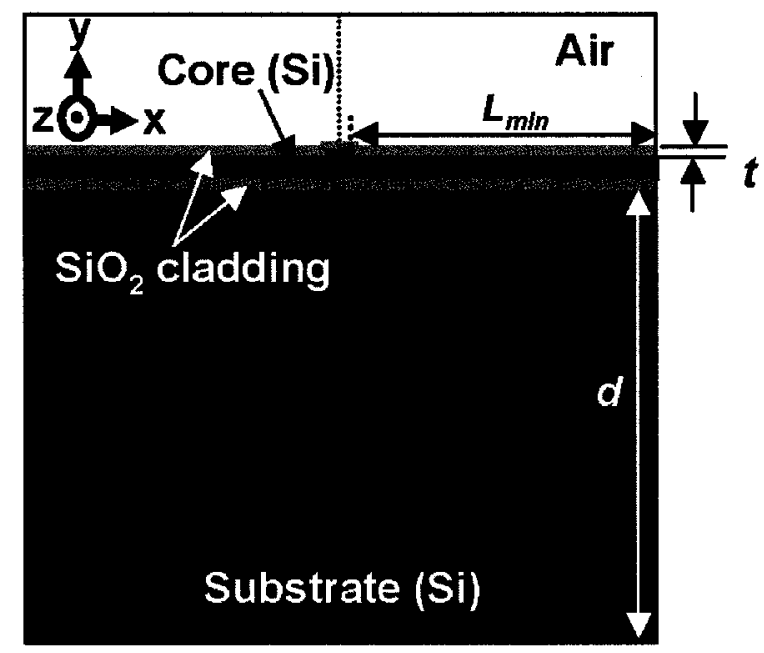

Figure 3.4: Simulation window size. The cladding film thickness is $t$, the substrate thickness is $d$. Usually $d \gg t$. The parameter $L_{\min }$ defines the minimum distance between the edge of the ridge waveguide and the calculation window boundary to avoid edge effects.

Since the strain $\varepsilon_{x}$ is virtually identical in the Si waveguide layer and the cladding for large bend radii, the stress relief $\Delta \sigma_{w}$ in the Si waveguide layer is related to the stress relief in the cladding by a ratio of the elastic constants:

$$
\Delta \sigma_{w}=\frac{E_{s}(1-\nu)}{E\left(1-\nu_{s}\right)} \Delta \sigma=-\frac{4 t \sigma_{0}}{d}
$$

Eq. 3.12 and 3.13 shows that the bending corrections are of the order of $t / d$, which implies that the film stress in the cladding and waveguide layer can vary significantly if the substrate thickness $d$ is comparable to the film thickness $t$, but may be neglected 
for thick substrates. Stress relief also arises from nonuniform strain relaxation at the edges of our finite model system. In a real system, the Si substrate is thick enough (e.g., $\sim 500 \mu \mathrm{m})$ and the edges are far away from the waveguide such that the stress relief due to edge effects and the wafer bending can be neglected. In numerical simulations, however, the modeled system should be as small as possible so that the FEM calculations can be completed in a reasonable amount of time and within the available memory allocation. In the conventional calculation of stress fields using FEM, the model wafer height and width must be large enough that stress relief due to strain relaxation at the sides of the wafer is negligible at the ridge waveguide, and the substrate is thick enough that stress relief from bending does not significantly modify the calculated film and waveguide stresses. Simulations show that the edge-induced distortion of the stress field extends almost 50 $\mu \mathrm{m}$ from the edge of the wafer for a $1 \mu \mathrm{m}$ thick oxide film, requiring that the calculation window be at least $2 L_{\min }=100 \mu \mathrm{m}$ wide, where $L_{\min }$ is defined in Fig. 3.4. Similarly, to minimize stress relief, the wafer thickness must be at least $90 \mu \mathrm{m}$ or larger. The typical $100 \mu \mathrm{m} \times 100 \mu \mathrm{m}$ window required for an accurate stress calculation is about 250 times larger than the window required for the calculation of the waveguide modes, as shown in Fig. 3.2.

The normalized plane strain model can effectively account for the thermal expansion along the $z$-axis $\left(\varepsilon_{z}=\alpha_{s} \Delta T\right)$, but both the ordinary and normalized plane strain models do not allow bending of the wafer along the $z$-axis because the strain component $\varepsilon_{z}$ has no dependence on either $x$ or $y$. Therefore stress relief due to wafer bending will not be correctly evaluated (see deviations in calculated stress levels and birefringence from the generalized model in Table 3.2). We can virtually eliminate the wafer bending and reduce the range of lateral edge effects by artificially setting the Young's modulus of the substrate wafer to a large value $E_{s}^{\prime}$. In the limit of a large Young's modulus, the edge distortion of the stress fields extends only about $15 \mu \mathrm{m}$ from the edge, while the bending contribution to stress relief is effectively eliminated since the wafer behaves as if it is 
absolutely rigid. Our calculations confirm that the stress relief effects are negligible for typical substrate thicknesses. Using a Young's modulus of $130 \mathrm{TPa}$ for the substrate ( $10^{3}$ times higher than the typical $E_{s}$ value without wafer bending), the film stress value differs by less than $1 \%$ of the expected value for a $500 \mu \mathrm{m}$ substrate with typical $E_{s}$, and less than $5 \%$ for a $100 \mu \mathrm{m}$ substrate, given the oxide film thickness of $1 \mu \mathrm{m}$. Since the film stress values are independent of the substrate thickness when a large $E_{s}^{\prime}$ is adopted, the modeled substrate thickness may be reduced from the initial $100 \mu \mathrm{m}$ to $5 \mu \mathrm{m}$ with no effect on the calculated stress fields in the ridge waveguide.

While the system size is greatly reduced by using a large $E_{s}^{\prime}$ with a thin substrate, the effect of stress relief cannot be reproduced. This is normally not an issue because the stress relief is quite small (of the order of $t / d$ ) and is neglected in most cases. However, in situations where stress relief effects need to be identified and accounted for the device performance, Eq. 3.12 and 3.13 can be used to determine a value for $E_{s}^{\prime}$ that will produce the same stress relief as for a wafer of a given thickness. Therefore, by using Eq. (3.12) to estimate the bend-induced strain that would occur for the real wafer, the effect of stress relief can be modeled by choosing the right combination of a modeled substrate thickness and an artificial values of the Young's modulus.

Table 3.3 makes a similar comparison as in Table 3.2, except the Young's modulus of the substrate $E_{s}$ is increased by 3 orders of magnitude to $130 \mathrm{TPa}$. The $100 \mu \mathrm{m}$ (wide) $\times 100 \mu \mathrm{m}$ (thick) window for stress calculations used for Table 3.2 is reduced to a much smaller computation window of $40 \mu \mathrm{m} \times 5 \mu \mathrm{m}$. The FEM calculations confirm that in the absence of wafer bending (i.e., high Young's modulus $E_{s}$ ), the results of the fully generalized plane strain model and the normalized plane strain model are in excellent agreement. The overall increase in birefringence of about $14 \%$ in Table 3.3 compared with the results presented in Table 3.2 is due to the absence of stress relief $\left(\Delta \sigma_{w}\right)$ in the Si waveguide layer, from the calculations using a stiff substrate. The correction is this large because a $100 \mu \mathrm{m}$ thick substrate was used in the original calculations leading 
to Table. 3.2. The results presented in Table. 3.3 are expected to be a much better approximation to a system with a more realistic $500 \mu \mathrm{m}$ substrate.

We conclude that, by using the normalized thermal expansion coefficient $\left(\alpha^{\prime}\right)$ in Eq. 3.10 for each layer, combined with the correction term to $\sigma_{z}$ from Eq. 3.11, the accuracy of the plane strain model is equivalent to that of the generalized model. By combining the normalized plane strain model with a high $E_{s}^{\prime}$ for the substrate to eliminate stress relief effects, we have been able to reduce the computation time and memory usage required for FEM calculations of the stress-induced birefringence by more than two orders of magnitude, while retaining the accuracy of a fully generalized plane strain model. This model is used for the stress analysis presented in the next chapters. A FEMLAB simulation program that implements the normalized plane strain model is included in Appendix A. 


\begin{tabular}{|c|c|c|c|c|c|}
\hline Etch Depth & Model & $\sigma_{x}(\mathrm{MPa})$ & $\sigma_{y}(\mathrm{MPa})$ & $\sigma_{z}(\mathrm{MPa})$ & Birefringence $\Delta n_{\mathrm{eff}}=n_{\mathrm{eff}}^{\mathrm{TM}}-n_{\mathrm{eff}}^{\mathrm{TE}}$ \\
\hline \hline \multirow{2}{*}{$D=1.2 \mu \mathrm{m}$} & Generalized Plane Strain (GPS) Model & -281 & 0.05 & -282 & 0.002859 \\
& Normalized Plane Strain (NPS) Model & -281 & 0.05 & -282 & 0.002858 \\
\hline \multirow{2}{*}{$D=1.34 \mathrm{~m}$} & GPS Model & -282 & 0.02 & -283 & 0.004162 \\
& NPS Model & -282 & 0.02 & -283 & 0.004161 \\
\hline \multirow{2}{*}{$D=1.5 \mu \mathrm{m}$} & GPS Model & -282 & 0.04 & -282 & 0.005407 \\
& NPS Model & -282 & 0.04 & -282 & 0.005407 \\
\hline
\end{tabular}

Table 3.3: Comparison of oxide film stress $\sigma_{\text {film }}$ and waveguide birefringence calculations from generalized and normalized plane strain models. The stress values are taken at the middle of the oxide film, $4 \mu \mathrm{m}$ away from the center of the ridge waveguide. Young's modulus of the substrate was set to $E_{s}^{\prime}=10^{3} E_{s}$. The SOI ridge waveguide has the same dimensions as in Table 3.2. The stress calculation window is reduced to $40 \mu \mathrm{m}$ wide by $5 \mu \mathrm{m}$ thick (from $100 \mu \mathrm{m} \times 100 \mu \mathrm{m}$ as in Table 3.2). 


\subsection{Summary}

This chapter introduced the concept of the stress and the photoelastic effect, and described the two commonly used analytical models for stress calculations: the generalized plane-strain and the ordinary plane-strain model. A new calculation scheme, the Normalized Plane-Strain Model, was then proposed. This model greatly improves the accuracy of stress and birefringence calculations, compared to the ordinary plane strain model, but yet requires the same modest computational resources as the ordinary plane strain model. Although the effect of wafer bending cannot be correctly characterized in the normalized plane strain model, our calculations confirm that the stress relief effects are negligible for typical silicon substrate thicknesses of $500 \mu \mathrm{m}$ or more. A further approximation with the Young's modulus of the substrate $E_{s}$ to be arbitrarily large was adopted, completely removing the wafer bending effects from the stress calculations. As a result, the nece-ssary computation window is reduced and the efficiency of the calculations are greatly improved. This is to the best of our knowledge, the first time such a simplified and accurate plane strain model has been presented for stress calculations in optical wave-guides. This newly implemented normalized plane-strain model forms the basis for the investigation of the stress-induced effects in this project. 


\section{Chapter 4}

\section{Stress Engineering - Stress and}

\section{Geometry Induced Effects}

This chapter presents a comprehensive study of the stress and geometrical contributions to polarization sensitivity in SOI ridge waveguides, using the newly developed Normalized Plane-Strain numerical model. The effects of stress on waveguide modal indices and birefringence, combined with the geometrical parameters such as the ridge shape, sidewall slopes, etch depth, width, height and the thickness of the upper oxide cladding film will be investigated individually. Let us first establish a firm understanding of the influence of geometrical parameters on polarization sensitivity.

\subsection{Influence of Geometrical Parameters}

This section focuses on the pure geometrical effects on waveguide birefringence. No stress is considered in this section. Throughout the numerical simulations, the operating wavelength is assumed to be $\lambda=1550 \mu \mathrm{m}$, and the silicon core thickness $H$ is set to $2.2 \mu \mathrm{m}$ unless specified otherwise. In the theoretical work in this thesis, the material refractive indices of the silicon and the oxide are assumed to be $n_{\mathrm{Si}}=3.476$ and $n_{\mathrm{SiO}_{2}}=$ 1.444, respectively. These material parameters are quoted from Landolt-Bornstein [84], 
the best available references that we are aware of. The lower oxide cladding which separates the waveguide core from the substrate is $0.37 \mu \mathrm{m}$, which is the nominal thickness of the SOI samples used for our experiments. The film thickness covering the ridge sidewalls is assumed to be $70 \%$ of that on the top of the waveguiding layer. This average ratio of sidewall to flat surface coverage was determined from the scanning electron microscope (SEM) images of waveguides with an oxide cladding deposited by PECVD (plasma enhanced chemical vapor deposition). An example of the oxide sidewall and surface coverage can be found in Fig. 4.1.

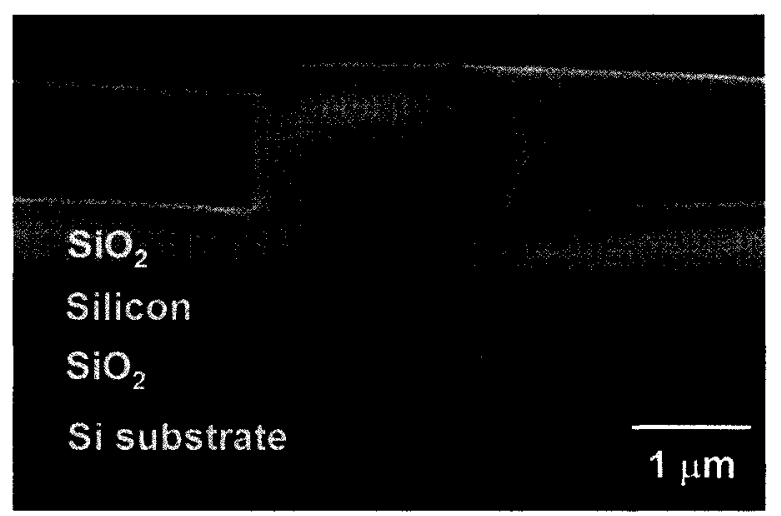

Figure 4.1: An SEM image of the cross section of a ridge waveguide in the arrayed grating section of a test AWG device (see Fig. 5.1).

\subsubsection{Effects of Upper Oxide Cladding}

The precise thickness of the buried oxide layer (lower cladding) is insignificant, as long as the optical field in the core cannot penetrate into the substrate. Similarly, the thickness of the upper oxide cladding film does not affect the effective indices of the TE and TM modes, as long as the thickness is higher than a few hundred nanometers. Fig. 4.2 shows the dependence of the effective index and the geometrical birefringence on the upper oxide cladding thickness. A trapezoidal ridge waveguide with sidewall slopes of $54^{\circ}$ is used for demonstration.

In the absence of stress, the modal properties are solely depended on the boundary conditions imposed by the right geometry. For the first $100 \mathrm{~nm}$ of the oxide cladding, 


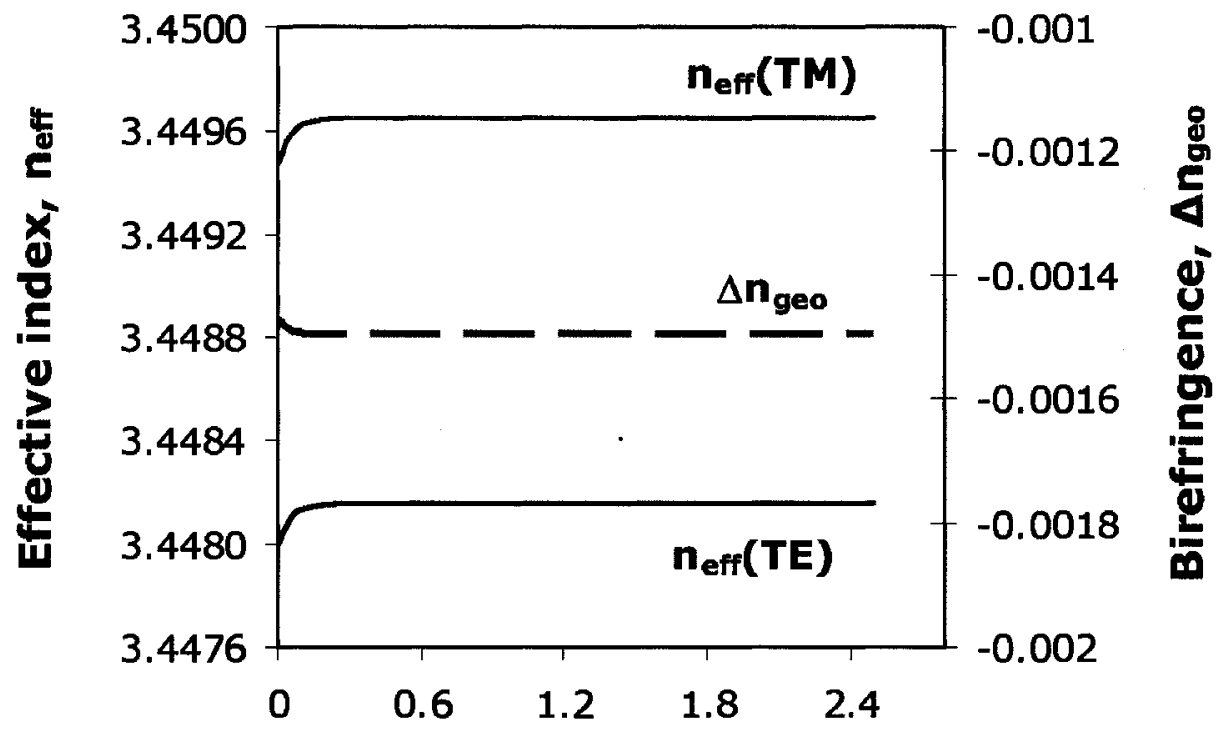

\section{Oxide thickness $\mathrm{t}(\boldsymbol{\mu m})$}

Figure 4.2: Influence of the upper oxide cladding thickness $(t)$ on the effective index $\left(n_{\mathrm{eff}}^{\mathrm{TM}}\right.$ and $\left.n_{\mathrm{eff}}^{\mathrm{TE}}\right)$ and the geometrical birefringence $\left(\Delta n_{\mathrm{geo}}=\right.$ $\left.n_{\mathrm{eff}}^{\mathrm{TM}}-n_{\mathrm{eff}}^{\mathrm{TE}}\right)$. The considered trapezoidal ridge waveguide has the following geometry parameters: $W_{1}=1.5 \mu \mathrm{m}$, sidewall slope $\theta=54^{\circ}, D=1.5 \mu \mathrm{m}$, and $H=2.2 \mu \mathrm{m}$. No stress is considered here.

the mode is slightly less confined since a small portion of the mode penetrates through the thin layer of the oxide cladding and couples into the surrounding air. The TM mode is more strongly affected than TE because of the change in the boundary conditions, resulting a positive shift of $1.8 \times 10^{-5}$ in $\Delta n_{\text {geo }}$. Both TE and TM effective indices soon saturate at the oxide thickness of $t \approx 0.1 \mu \mathrm{m}$. The overall birefringence remains at around $1.50 \times 10^{-3}$, as illustrated in Fig. 4.2. A similar trend is observed for the ridge waveguides with vertical sidewalls and even slab waveguides. It is noted that thinner waveguide cores (i.e., small $H$ ) require a thicker oxide upper and lower cladding to ensure sufficient mode confinement. For the waveguides studied throughout this project, our silicon core has a thickness of $2.2 \mu \mathrm{m}$, and the oxide cladding is usually much thicker than $0.3 \mu \mathrm{m}$. Based on these parameters, the overall geometrical birefringence $\Delta n_{\text {geo }}$ for our devices is independent of the thickness of the upper oxide cladding. 


\subsubsection{Effects of Ridge Geometrical Profile}

Two types of waveguide cross-sectional profiles are considered here: the rectangular (vertical-sidewall), and the trapezoidal (slanted-sidewall) ridge waveguides. The two profiles are obtained by using dry and wet etching, respectively. Dry-etch processing, due to its higher operational cost, is usually used when the feature resolution in thin film structures is of a major concern or when deep vertical sidewall etchings are required. Wet-etching, on the other hand, is simpler, yet yields resolution sufficient for many waveguide devices. Another important advantage for wet-etching is that it tends to produce smoother sidewalls as compared to RIE (reactive ion etching) and thus scattering loss is significantly reduced $[89,90]$.

Although the comparison criteria of these two rather different types of waveguides are not obvious, we adopt the geometry parameters produced from an identical design layout. That is, the two types of waveguides have the same targeted etch depth and core thickness; however, depending on the etching technique, the waveguides will have different cross-sections. Typically, dry-etching produces nearly vertical sidewalls $\left(90^{\circ} \pm 3^{\circ}\right.$ in our fabrication laboratory), while the wet-etching yields approximately $54^{\circ}$ sidewalls (the angle between Si crystal $<111>$ and $<100>$ planes). Fig. 4.3 shows the equal-field contours of the fundamental TE mode in a rectangular (Fig. 4.3(a)) and a trapezoidal (Fig. 4.3(b)) ridge waveguide. Both waveguides have a top width of $2 \mu \mathrm{m}$ and an etch depth of $1.5 \mu \mathrm{m}$, one with a vertical sidewall slope $\left(90^{\circ}\right)$, and the other with a $54^{\circ}$ sidewall. The abrupt changes in the mode shape due to the sharp inner corners at the silicon and oxide layer boundary of the rectangular waveguide core contrast distinctively with the much smoother field contours in the trapezoidal waveguide. The waveguide effective indices and birefringence of the two cases are quite different $\left(n_{\text {rect }}^{\mathrm{TE}}=3.440592\right.$ and $n_{\text {trape }}^{\mathrm{TE}}=3.452536 ; \Delta n_{\text {rect }}=-5.7 \times 10^{-5}$ and $\Delta n_{\text {trape }}=-1.59 \times 10^{-3}$ ). The waveguide changes from a low birefringent waveguide to a highly birefringent one, with a drastic increase in birefringence of $\Delta n_{\text {rect }}-\Delta n_{\text {trape }}=1.5 \times 10^{-3}$. We can conclude that, despite 


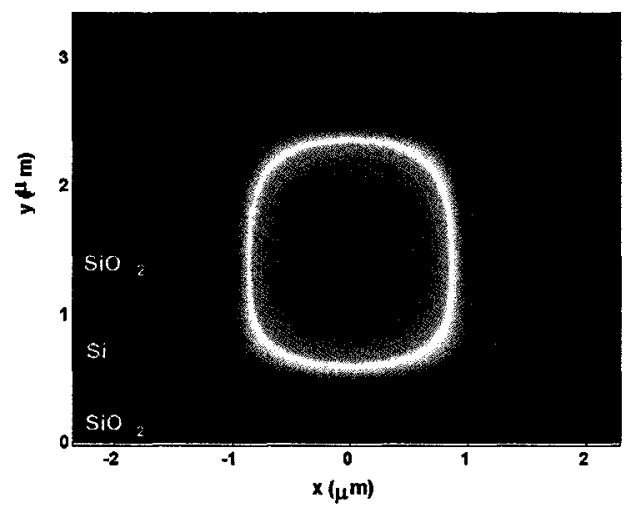

(a)

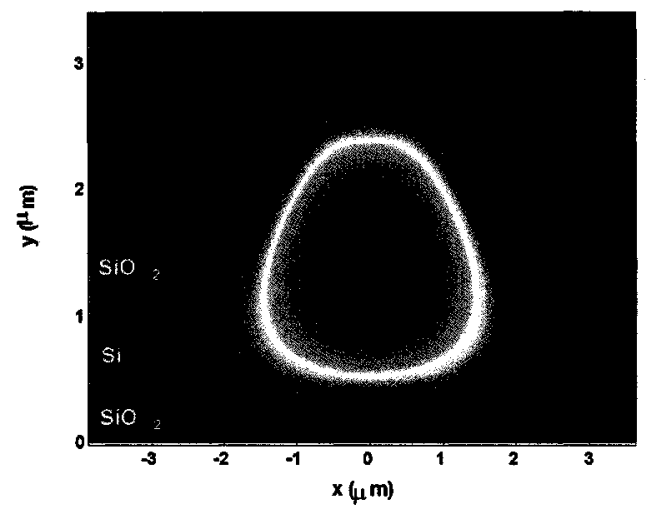

(b)

Figure 4.3: Fundamental TE mode shapes with equal electric field contours $(0.5,0.1$, and 0.01 of the maximum fields) in SOI ridge waveguide. The ridge height is $2.2 \mu \mathrm{m}$, the etch depth is $1.5 \mu \mathrm{m}$. (a) A rectangular ridge with a width of $2 \mu \mathrm{m}$; and (b) a trapezoidal ridge with a top width $W_{1}$ of $2 \mu \mathrm{m}$ and a sidewall angle of $54^{\circ}$.

the similar mode guiding ability, the optical properties of rectangular and trapezoidal waveguides can be significantly different.

Since the sidewall slopes of a fabricated waveguide are determined by the specific etching technique or the waveguide orientation with respect to the crystallographic planes, it is not trivial to tune the sidewall angles in practice. Here we limit our discussion to a theoretical influence of sidewall slope angles on polarization sensitivity. To determine the effects of the ridge sidewall slope, simulations have been carried out for etch depths of $1.3,1.5$ and $2 \mu \mathrm{m}$, assuming the upper ridge width $\left(W_{1}\right)$ is fixed to $1.5 \mu \mathrm{m}$.

Fig. 4.4 plots the geometrical birefringence as a function of ridge sidewall slope. For a given etch depth, the rate of change in waveguide birefringence $\left(\frac{\partial(\Delta n)}{\partial \theta}\right)$ increases with the ridge sidewall slope $\theta$. As shown in Fig. 4.4, the etch depth plays a critical role in determining birefringence, especially for ridges with large sidewall angles such as rectangular waveguides. Waveguides with smaller sidewall angles have better birefringence stability to etch depth variations; however, these waveguides have relatively large negative birefringence. In other words, the geometrical birefringence of a trapezoidal ridge waveg- 


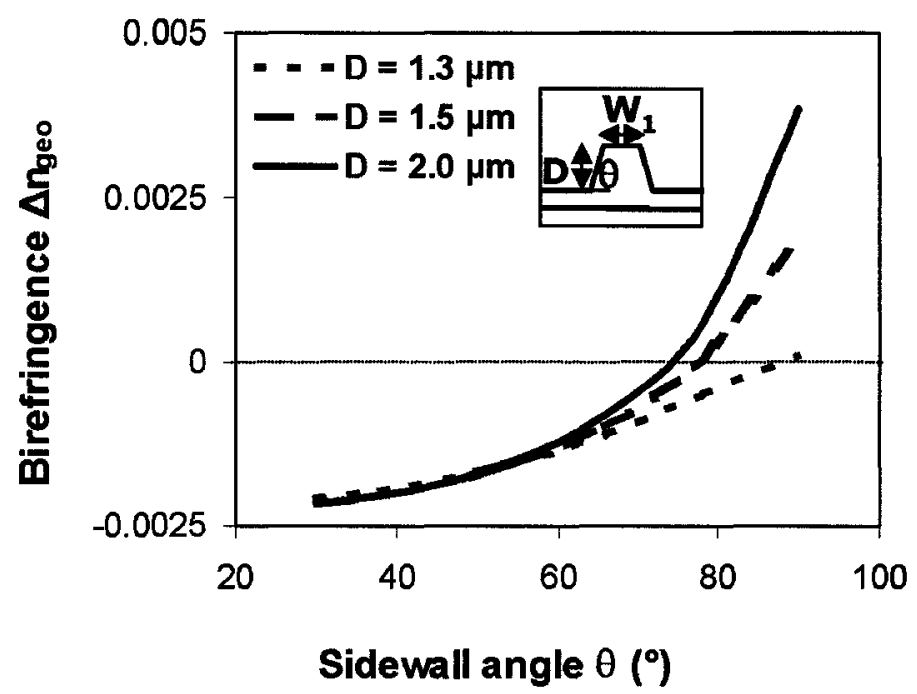

Figure 4.4: The variation of waveguide geometrical birefringence $\left(\Delta n_{\text {geo }}\right)$ with sidewall slope $(\theta)$ for 3 different etch depths $(D)$. The Si ridge has an upper width $\left(W_{1}\right)$ of $1.5 \mu \mathrm{m}$ and the total height of the core is $2.2 \mu \mathrm{m}$.

uide is less susceptible to changes in the ridge geometry as compared to a rectangular waveguide.

The influence of etch depth on birefringence is further shown in Fig. 4.5 for four different sidewall angles of $54^{\circ}, 72^{\circ}, 87^{\circ}$, and $90^{\circ}$, with a fixed upper ridge width $W_{1}=1.5 \mu \mathrm{m}$ and a ridge height of $H=2.2 \mu \mathrm{m}$. In the numerical simulations, we use the experimentally measured $87^{\circ}$ (produced in our fabrication laboratory) and the ideal $90^{\circ}$ sidewall angles for rectangular ridge waveguides, $54^{\circ}$ (in-house measurement) and $72^{\circ}$ for trapezoidal waveguides. Again, it is observed that the birefringence is less sensitive to changes in etch depth for waveguides with trapezoidal cross-sections as compared to the rectangular ridge waveguides. However, the trapezoidal ridge waveguides have large negative birefringence, and for the given upper ridge width, the birefringence-free point cannot be reached for any choice of etch depth. This is not the case in waveguides with nearly vertical sidewalls, whereas optimal etch depth can be found to satisfy the birefringence-free condition (zero birefringence at $\sim 1.3 \mu \mathrm{m}$ ). Therefore a precise control of the etch depth is necessary to achieve polarization independent operation in the rectangular waveguides. For a given ridge width, a fluctuation of $0.1 \mu \mathrm{m}$ in the etch depth could result in a change 
of $10^{-3}$ in birefringence, as shown in Fig. 4.5.

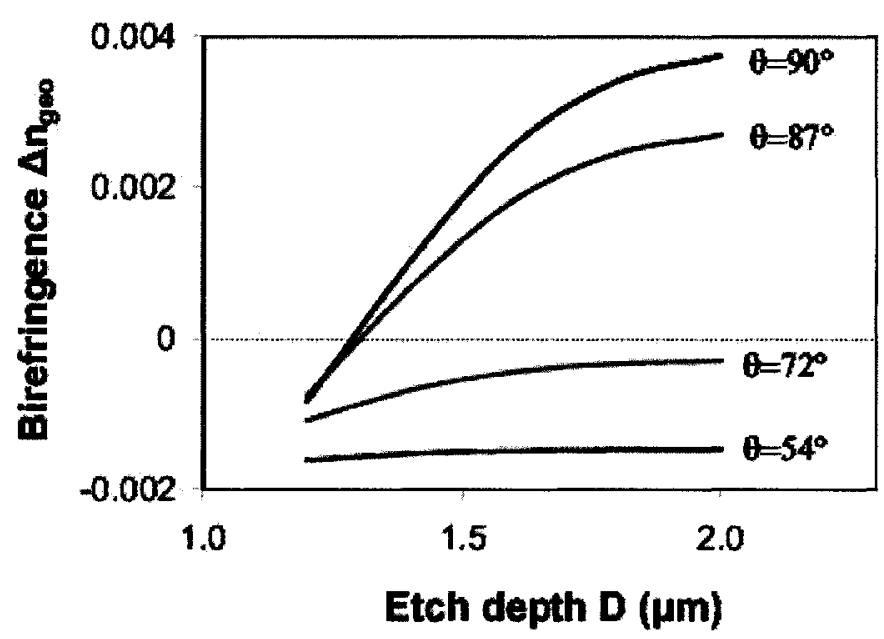

Figure 4.5: Etch depth influence on waveguide geometrical birefringence $\left(\Delta n_{\text {geo }}\right)$ for rectangular and trapezoidal waveguides with sidewall slope of $54^{\circ}, 72^{\circ}, 87^{\circ}$ and $90^{\circ}$, in the absence of stress. The Si ridge has a height $H$ of $2.2 \mu \mathrm{m}$ and a top ridge width $W_{1}$ of $1.5 \mu \mathrm{m}$ (bottom ridge width $W_{2}$ varies according to slope $\theta$ ).

The analyses presented thus far have always assumed a constant upper ridge width. To investigate the effects of ridge width on waveguide birefringence, simulations have been carried out by varying the widths $\left(W_{1}\right.$ and $\left.W_{2}\right)$, while maintaining all the other geometrical parameters fixed. Fig. 4.6 illustrates the influence of ridge width on waveguide effective index and birefringence for trapezoidal and rectangular ridge waveguides. The etch depth is fixed at $1.5 \mu \mathrm{m}$, and the core thickness is $2.2 \mu \mathrm{m}$.

It is apparent from Fig. 4.6(a) that both the effective index and the geometrical birefringence are less sensitive to the ridge width fluctuations for trapezoidal ridge waveguides than for rectangular waveguides, but trapezoidal ridge waveguides suffer a large negative geometrical birefringence. For a variation within $\pm 0.1 \mu \mathrm{m}$ in the top ridge width of $1.5 \mu \mathrm{m}$, the change for the rectangular waveguide in the effective index and in the geometrical birefringence are $8.4 \times 10^{-3}$ and $1.2 \times 10^{-3}$, respectively. In contrast, the corresponding changes for the trapezoidal waveguide (with a $54^{\circ}$ sidewall angle) in $n_{\text {eff }}^{\mathrm{TE}}$ and $\Delta n_{\text {eff }}$ are $1.5 \times 10^{-3}$ and $7.0 \times 10^{-5}$, respectively. Thus, the ridge waveguides with a 


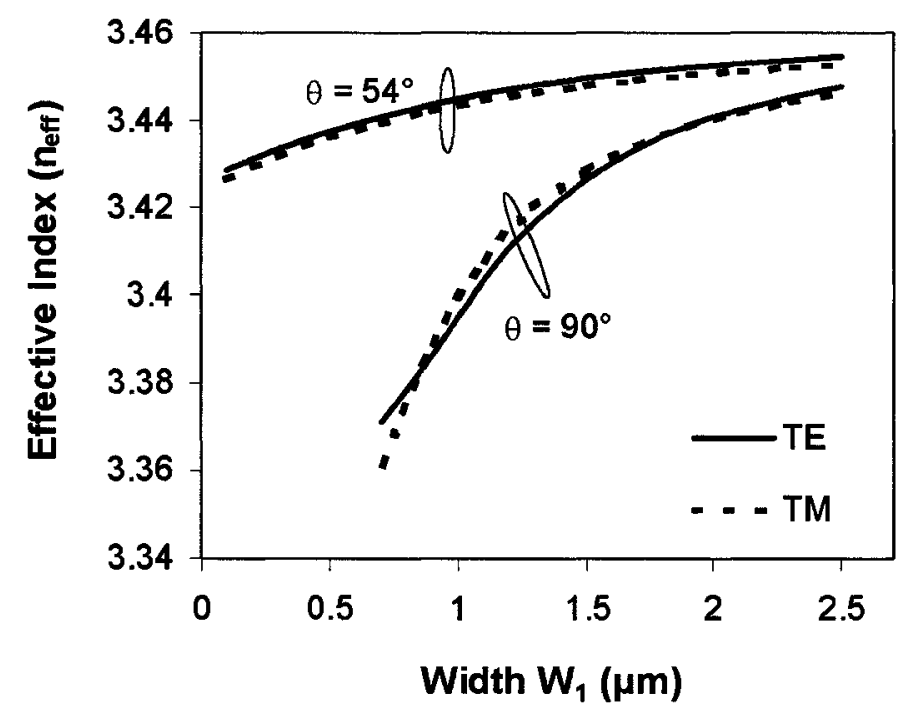

(a)

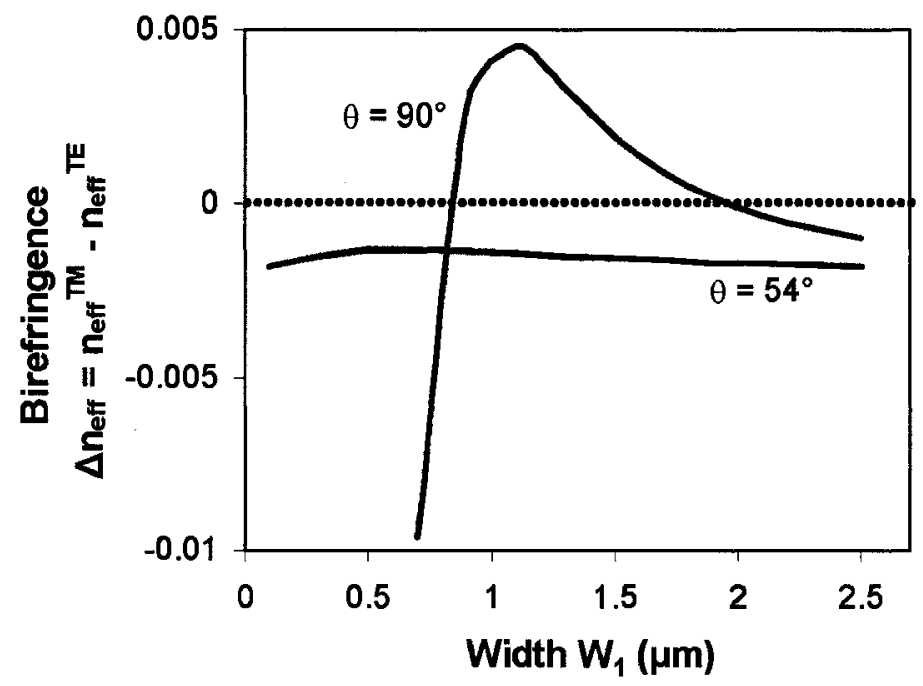

(b)

Figure 4.6: Ridge width influence on waveguide effective index and geometrical birefringence for trapezoidal and rectangular waveguides. Shown are (a) effective mode indices of TE and TM polarizations in trapezoidal $\left(\theta=54^{\circ}\right)$ and rectangular $\left(\theta=90^{\circ}\right)$ ridge waveguides and $(\mathrm{b})$ geometrical birefringence $\left(\Delta n_{\text {geo }}\right)$. The Si ridge etch depth is fixed at $1.5 \mu \mathrm{m}$, and ridge height $H$ is $2.2 \mu \mathrm{m}$.

trapezoidal cross-section are more tolerant to errors in waveguide dimensions.

The effective index of the TE and TM modes increase with the ridge width for both the trapezoidal and rectangular ridge waveguides, implying a stronger confinement of 
the waveguide mode in wide ridge waveguides. In fact, the effective index and the birefringence of the two types of waveguides are observed to converge to a common value as shown in Fig. 4.6. As the width increases to the infinity, the values of the effective index and the birefringence in both types of waveguides approach to that of a $2.2 \mu \mathrm{m}$ slab waveguide (i.e., $n_{\text {slab }}^{\mathrm{TE}}=3.460405$ and $\Delta n_{\text {slab }}^{\text {geo }}=-0.001867$ ). At the other extreme, however, as the ridge width approaches zero, the difference in effective index and birefringence between the two types of waveguides becomes the largest. Fig. 4.7 illustrates the mode profiles of the two types of the waveguides with a small ridge top width of $W_{1}=0.1 \mu \mathrm{m}$. The rectangular waveguide effectively becomes a $0.7 \mu \mathrm{m}$ slab waveguide (i.e., $n_{\text {slab }}^{\mathrm{TE}}=3.356658$ and $\Delta n_{\text {slab }}^{\text {geo }}=-0.047425$ ), while the trapezoidal waveguide evolves into a triangular profile retaining the $1.5 \mu \mathrm{m}$ etch depth.

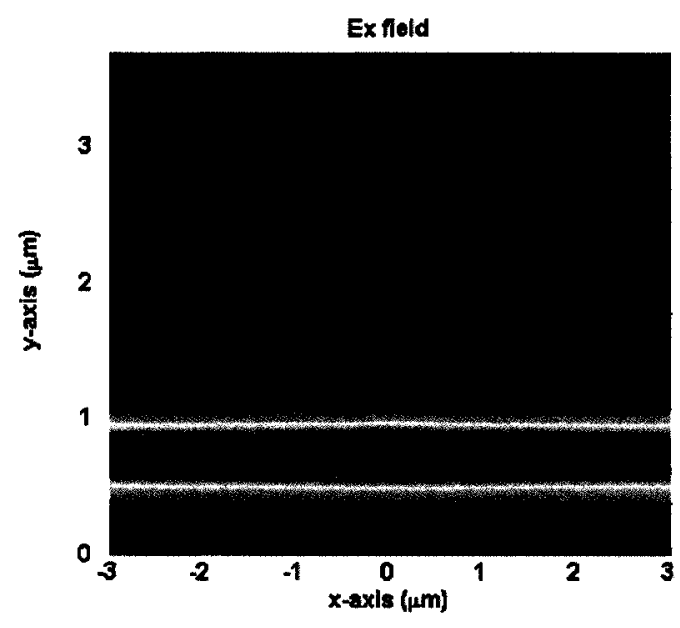

(a)

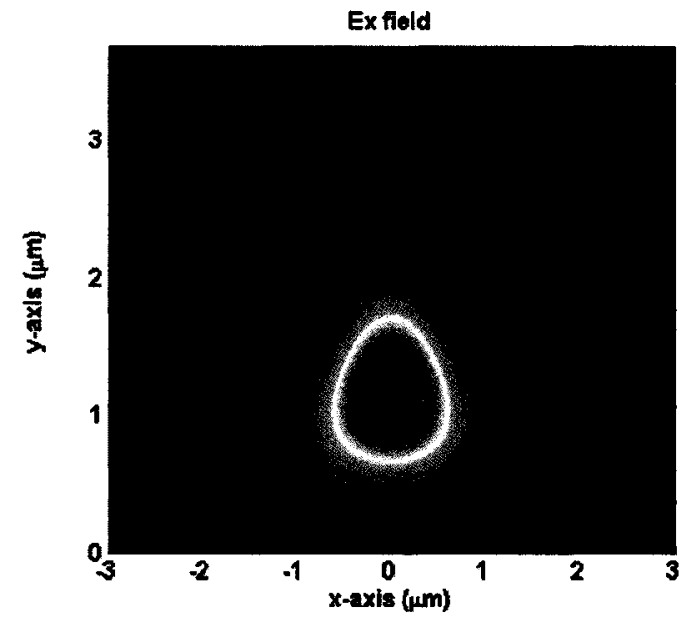

(b)

Figure 4.7: Electric field distribution of the fundamental TE mode for (a) a rectangular waveguide and (b) a trapezoidal waveguide with a sidewall angle of $54^{\circ}$. Both waveguides have $W_{1}=0.1 \mu \mathrm{m}, D=1.5 \mu \mathrm{m}, H=2.2 \mu \mathrm{m}$.

Fig. 4.8 summarizes the effects of ridge profile on waveguide birefringence. The geometrical birefringence $\Delta n_{\text {geo }}$ in a rectangular ridge waveguide has shown a strong dependence on the ridge aspect ratio $r$ where $r=1-D / H$. Here the silicon core thickness $H$ is fixed for all the simulation curves. One main observation is the convergence of 


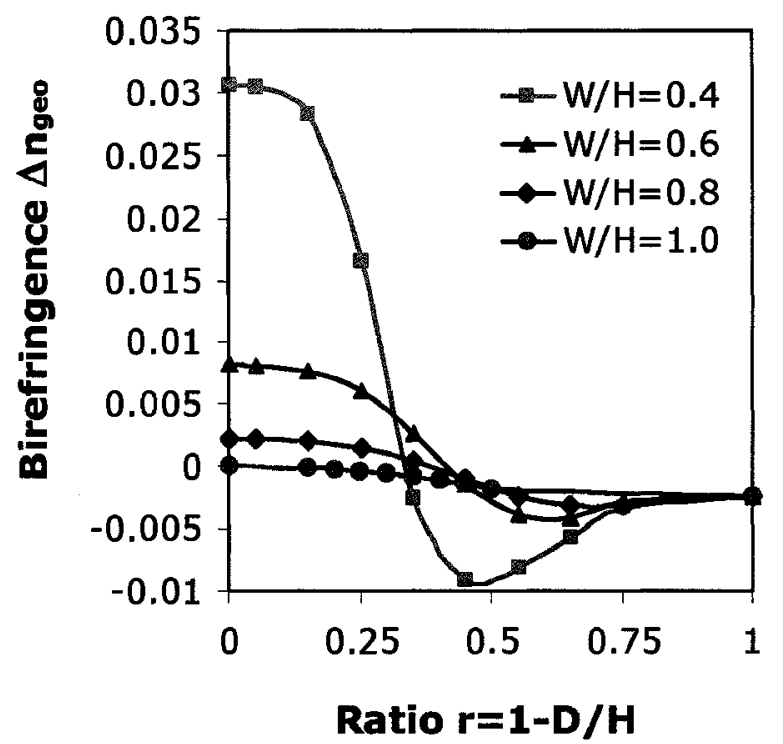

Figure 4.8: Geometrical birefringence $\Delta n_{\text {geo }}$ as a function of the parameter $r=1-D / H$ and the cross-section aspect ratio $W / H$ in rectangular ridge waveguides. Here the silicon core thickness $H$ is fixed at $2 \mu \mathrm{m}$.

$\Delta n_{\text {geo }}$ for an arbitrary ridge profile $W / H$ at $r=1$ (or zero etch depth $D=0$ ). Shallow etched waveguides (with large ratios $r$ ) usually have a negative $\Delta n_{\text {geo }}$ because the mode is weakly confined in the ridge and is similar to a slab mode. As the ratio $r$ decreases (for deeply etched ridges), the mode becomes more confined in the waveguide, shifting $\Delta n_{\text {geo }}$ to a more positive value. As the ratio $r$ becomes 0 where the etch depth is the deepest, the optical modes are confined the most, and $\Delta n_{\text {geo }}$ achieves a maximum value. It is apparent that the geometrical birefringence is more sensitive to geometrical variations for narrower ridge structures (with small $W / H$ ratios). To achieve an acceptable level of birefringence required in the state-of-the-art photonic devices $\left(\Delta n_{\text {geo }}<10^{-5}\right)$, the ridge cross-section aspect ratio $W / H$ must be adjusted accordingly, with a dimension control on the order of $10 \mathrm{~nm}$. 


\subsubsection{Effects of Ridge Height}

Next, the effects of the ridge height $(H)$ on geometrical birefringence are investigated. Fig. 4.9 shows the dependence of the birefringence on the ratio $r$ for rectangular waveguides of varying ridge heights of $1,2,3$, and $5 \mu \mathrm{m}$, respectively. The geometry is chosen to have a ridge width that is smaller than the core thickness (i.e., $W \leq H$ ), such that a zero geometrical birefringence point may be obtained. These curves show the dependence of $\Delta n_{\text {geo }}$ on etch depth, which is consistent with the information obtained in Figs. 4.5 and 4.8. It is worth noting that the ratio $r$ which gives zero birefringence increases with the ridge height $(H)$.

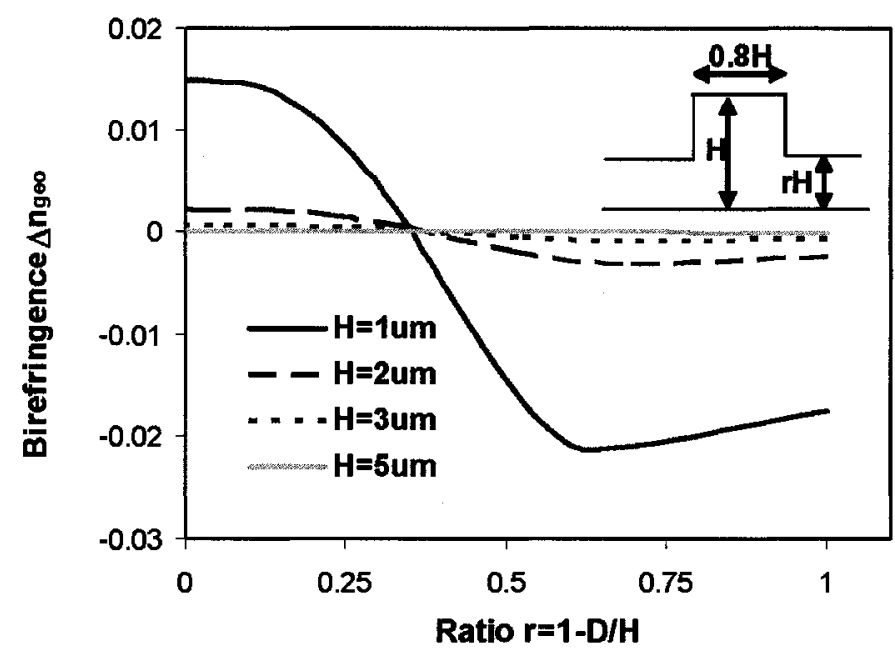

Figure 4.9: Geometrical birefringence $\Delta n_{\text {geo }}$ as a function of the ridge etch ratio $r$, for different ridge height $H$ as indicated in the figure. The waveguide geometry is shown in the inset, with $W / H=0.8$.

The variations in geometrical birefringence as a function of ratio $r$ increase as the core thickness decreases, similar to what have been observed in Fig. 3.1. For large ridge heights such as $H=4 \mu \mathrm{m}$ shown in Fig. 4.9, the geometrical birefringence remains around $1 \times 10^{-4}$ for a considerable range of $r$. The variation in birefringence is $\pm 5 \times 10^{-5}$ for a change of 0.1 in $r$. In contrast, the changes in birefringence in smaller ridge heights are much larger, with a fluctuation of $\pm 1.5 \times 10^{-3}$ for the same 0.1 variation in $r$ at $H=1 \mu \mathrm{m}$. As a result, the usable range of $r$ which corresponds to the birefringence-free 
propagation in ridge waveguides with a small height $H$ is extremely narrow, compared to waveguides with a larger ridge height.

\subsubsection{Summary}

In this section, the geometrical contributions to the waveguide birefringence were investigated. It has been shown that although in principle the birefringence-free and single-mode conditions can be simultaneously achieved for waveguides with large cross-sections by adjusting the etch depth, width, or the height of the ridge geometry, this approach is impractical for small waveguides with dimensions in the order of a micron as the waveguide performance becomes extremely sensitive to fluctuations in geometrical parameters. The study has focused on ridge waveguides with small cross-sections in two types of geometries, the rectangular and the trapezoidal profile, respectively. The waveguides with trapezoidal ridge cross-sections or with wide ridge widths, offer improved birefringence tolerance to dimension fluctuations. However, the trapezoidal waveguides are associated with large negative birefringence.

\subsection{Stress-induced Birefringence}

Fig. 4.10 compares the stress distributions in a rectangular waveguide with that in a trapezoidal waveguide. The deposition temperature of the oxide cladding layer for both cases is assumed to be identical at $1000^{\circ}$. Clearly, the stress distribution in a waveguide is dependent on the cross-section geometry. With the knowledge of the geometry-induced effects on waveguide birefringence from the previous section, we now investigate the combined stress and geometry induced effects.

The stress-induced birefringence is produced by the photoelastic effect due to an asymmetric stress in the core. We use only the thermal model for stress calculations in simulation, because the thermal stress is the largest contribution to the total stress in 


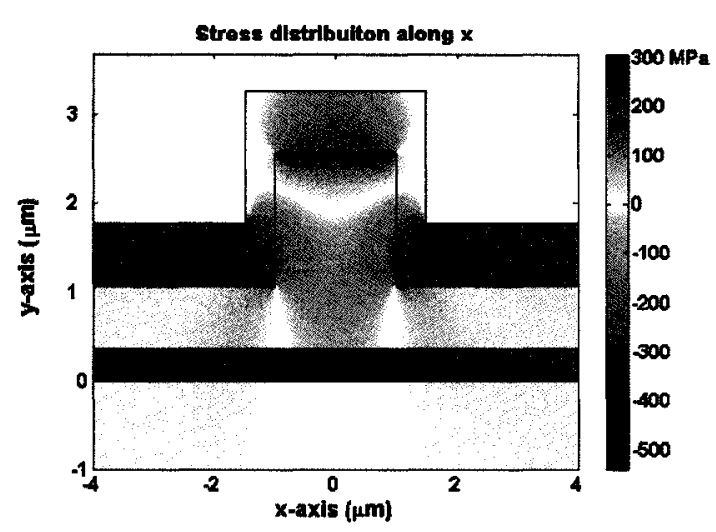

(a)

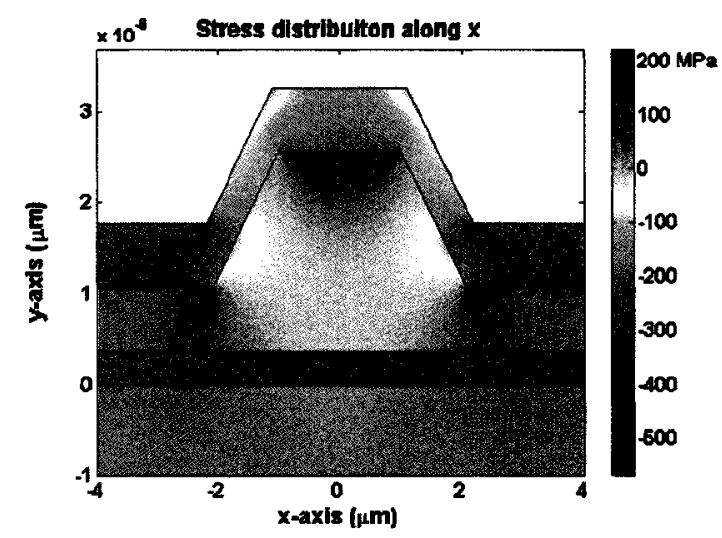

(b)

Figure 4.10: Stress distributions along $x$-direction $\left(\sigma_{x}\right)$ for (a) a rectangular waveguide and (b) a trapezoidal waveguide with a sidewall angle of $54^{\circ}$. Both waveguides have $W_{1}=1.5 \mu \mathrm{m}, D=1.5 \mu \mathrm{m}$, and $H=2.2 \mu \mathrm{m}$. The oxide cladding thickness is $t=0.7 \mu \mathrm{m}$.

a waveguide system, which is due to the mismatch in the thermal expansion coefficients between the core and the oxide cladding. The thermal expansion coefficient of silicon is approximately seven times higher than that of $\mathrm{SiO}_{2}$, a compressive stress is developed in the oxide cladding during the cooling process after the oxide deposition step under an elevated temperature. The stress in a material can be altered by changing the thermal annealing conditions [32] or by modifying material properties. The cladding thickness can be precisely controlled during the fabrication process by deposition or etching. Adjusting the stress in the upper cladding layer affects the magnitude of the stress-induced birefringence, which can be used to control and eliminate the overall waveguide birefringence.

As defined in the previous chapter, the stress parameter $\sigma_{\text {film }}$ is the in-plane stress component (in the $x$ direction) that is present in a two-dimensional uniform thin oxide film, far away from the ridge. Although this parameter has been chosen to quantify and characterize the stress in a cladding film of a ridge waveguide, the actual stress distributions in the vicinity of a ridge waveguide are inhomogeneous (as seen in Fig. 4.10) and dependent on the ridge cross-sectional geometry. 


\subsubsection{Effects of Oxide Cladding Stress}

\section{Cladding Thickness}

Fig. 4.2 illustrates the independence of the waveguide geometrical birefringence $\Delta n_{\text {geo }}$ on the oxide cladding thickness, in the absence of stress. However, with the inclusion of the stress-induced effects, the effective indices of the TE and TM modes will be modified. As predicted by Eq. 3.1, a negative shift in the material index $n_{x}$ and a positive shift in $n_{y}$ are induced by a compressive stress (i.e., negative stress value) in the cladding film. These changes in turn modify the TE and TM modal effective indices. As shown in Fig. 4.11, for a given waveguide geometry, $n_{\mathrm{eff}}^{\mathrm{TE}}$ decreases with the oxide thickness and the stress magnitude, while $n_{\mathrm{eff}}^{\mathrm{TM}}$ increases. The stress-induced shifts $\left(\delta n_{\text {stress }}\right)$ are stronger in

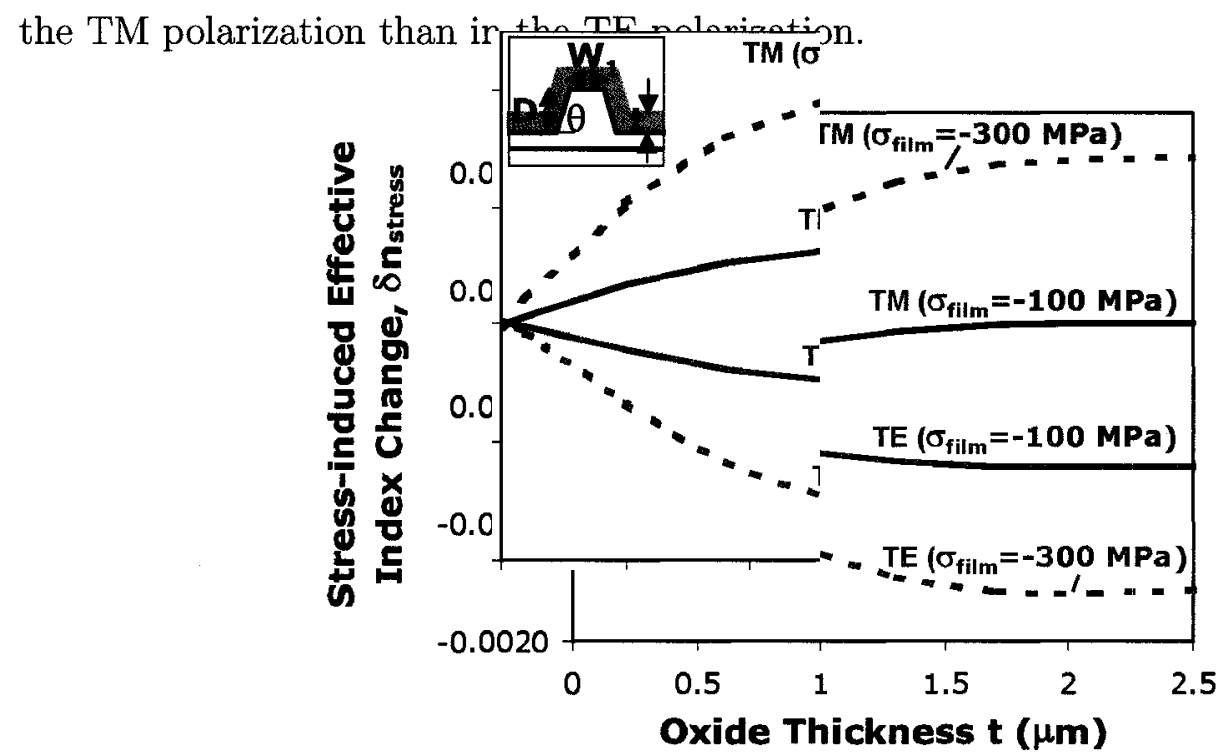

Figure 4.11: Influence of the upper cladding with different levels of stress on the effective indices of the TE and TM modes. The trapezoidal waveguide geometry is identical to that defined in Fig. 4.2: $\theta=54^{\circ}, W_{1}=1.5 \mu \mathrm{m}$, $D=1.5 \mu \mathrm{m}$, and $H=2.2 \mu \mathrm{m}$.

Fig. 4.12 shows the influence of stress on the total waveguide birefringence of the same structure used in both Fig. 4.2 and 4.11, for different stress levels in the oxide cladding: $\sigma_{\text {film }}=0,-100,-200$, and $-300 \mathrm{MPa}$. The bottom dashed curve corresponds to the geometrical birefringence ( $\Delta n_{\text {geo }}$, without stress) associated with the given waveguide 


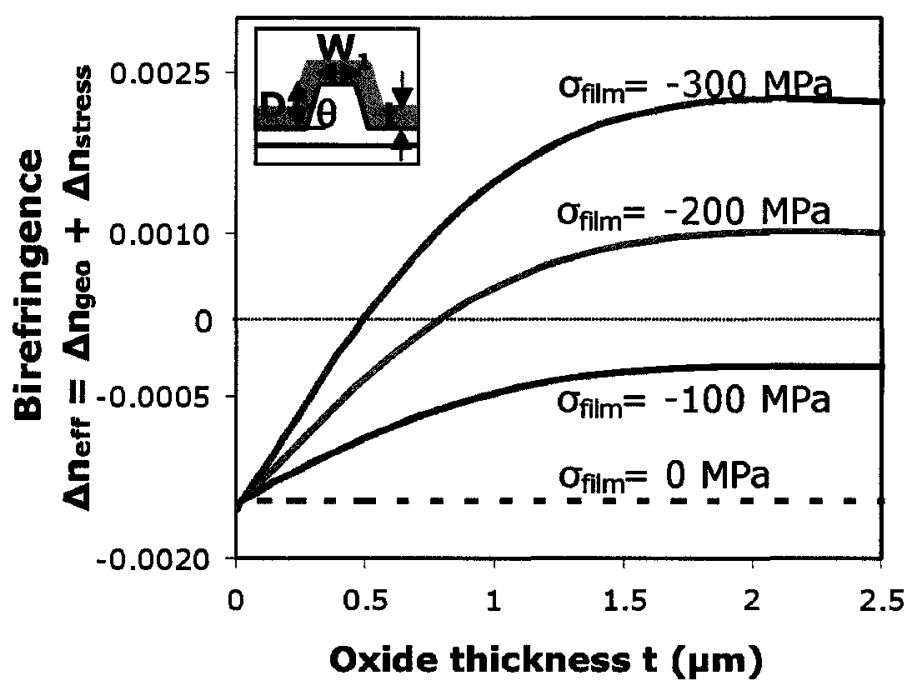

Figure 4.12: Influence of upper cladding under different levels of stress on waveguide birefringence. The bottom curve corresponds to the geometrical birefringence (no stress is included in the calculations of waveguide effective indices). The waveguide geometry is identical to that defined in Fig. 4.2 and 4.11 .

geometry, which is the same birefringence curve presented in Fig. 4.2. For this waveguide, the geometrical birefringence $\Delta n_{\text {geo }}$ remains constant at $-1.50 \times 10^{-3}$, and it was shown in Fig. 4.5 that this $\Delta n_{\text {geo }}$ cannot be eliminated by changing etch depths. However, with the inclusion of stress-induced effects, the birefringence can be effectively modified. The oxide cladding with a compressive stress induces a positive offset to the geometrical birefringence, giving a tuning range of $4.0 \times 10^{-3}$ for $\sigma_{\text {film }}=-300 \mathrm{MPa}$. In the example illustrated in Fig. 4.12, with an oxide cladding under a -200 MPa compressive stress, the TE and TM mode become degenerate when the film thickness is $t=0.80 \mu \mathrm{m}$ and the waveguide has a zero overall birefringence. It should be pointed out that this is not the only possible birefringence-free condition. Fig. 4.12 shows another birefringencefree condition with a cladding thickness of $t=0.50 \mu \mathrm{m}$ under a film stress of $\sigma_{\text {film }}=$ $-300 \mathrm{MPa}$. In fact, appropriate combinations of the oxide thickness $(t)$ and the stress level $\left(\sigma_{\text {film }}\right)$ create a wide range of conditions for zero overall birefringence in a given waveguide geometry. One interesting observation is that the stress-induced changes in effective index $\left(\delta n_{\text {stress }}\right)$ and waveguide birefringence $(\Delta n)$ scale linearly with the stress 
in the cladding film $\left(\sigma_{\text {film }}\right)$. Evidently, the cladding thickness $(t)$ and the film stresses $\left(\sigma_{\text {film }}\right)$ are two parameters which can effectively tune the stress-induced birefringence. Depending on the initial value of the geometrical birefringence which is purely geometry dependent, the magnitude of the total birefringence may be compensated, either reduced or increased, with proper choices of $t$ and $\sigma_{\text {film }}$.

\section{Temperature Sensitivity}

The oxide cladding is assumed to be deposited at a temperature $T_{0}=400^{\circ} \mathrm{C}$, then it is cooled down to room temperature $\left(20^{\circ} \mathrm{C}\right)$ for use. In the previous simulations, the actual operating temperature was always assumed to be room temperature. However, in reality, the operating temperature fluctuates due to the surrounding external environments. Fig. 4.13 presents a study of the temperature sensitivity of the stress engineering within the operation range from 10 to $80^{\circ} \mathrm{C}$.

The stress is expected to be dependent on the temperature change $(\Delta T)$, as expressed in Eq. 3.4. Fig. 4.13(a) shows the stress as a function of temperature, with a rate of change of $\Delta \sigma_{\text {film }} / \Delta T=0.27 \mathrm{MPa} / 1^{\circ} \mathrm{C}$. The corresponding changes in the stress-induced effective indices of TE and TM, and the stress-induced birefringence, with respect to the temperature variations, are plotted in Fig. 4.13(b). The change in stressed-induced birefringence $\Delta n_{\text {stress }}$ is $-4.6 \times 10^{-6} / 1^{\circ} \mathrm{C}$, while the rate of change in $\delta n_{\text {stress }}^{\mathrm{TE}}$ and $\delta n_{\text {stress }}^{\mathrm{TM}}$ are $1.8 \times 10^{-6} / 1^{\circ} \mathrm{C}$ and $-2.7 \times 10^{-6} / 1^{\circ} \mathrm{C}$, respectively. All are on the order of $10^{-6}$ per $1^{\circ} \mathrm{C}$ change in temperature. However, the optical performance of the waveguide device can significantly change for large temperature fluctuations. For example, a change in $100^{\circ} \mathrm{C}$ in operating temperature would result in a $-0.17 \mathrm{~nm}$ change in the polarization dependent peak wavelength shift of a polarization insensitive SOI AWG spectrometer which is optimized at room temperature; that is, $\lambda_{\mathbf{p}}^{\mathrm{TM}}-\lambda_{\mathrm{p}}^{\mathrm{TE}}$ changes from $0 \mathrm{~nm}$ to -0.17 $\mathrm{nm}$. In addition, for a polarization splitter device described in Chapter 6, a temperature fluctuation of $100^{\circ} \mathrm{C}$ would change the stress-induced index ratio $\frac{\delta n^{\mathrm{TE}}}{\delta n^{\mathrm{TM}}}$ from -0.6822 to - 
0.6834 , resulting in deterioration of the splitting performance (the extinction ratio reduces from $-20 \mathrm{~dB}$ down to $-6 \mathrm{~dB}$ ). Since many photonic devices require temperature control to achieve acceptable performance, similar temperature control design can be adopted for the stress-induced devices to accommodate large temperature fluctuations.

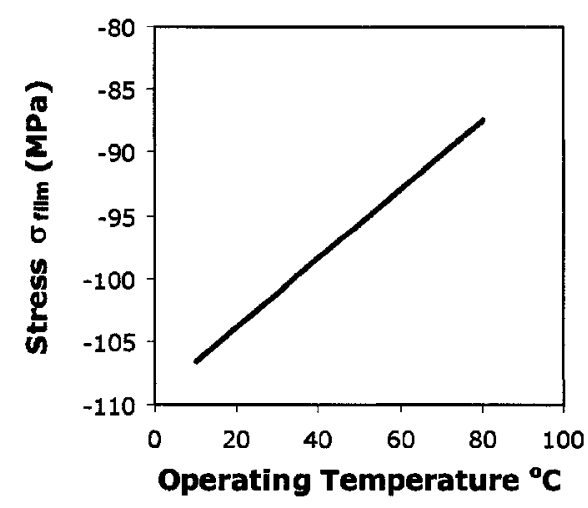

(a)

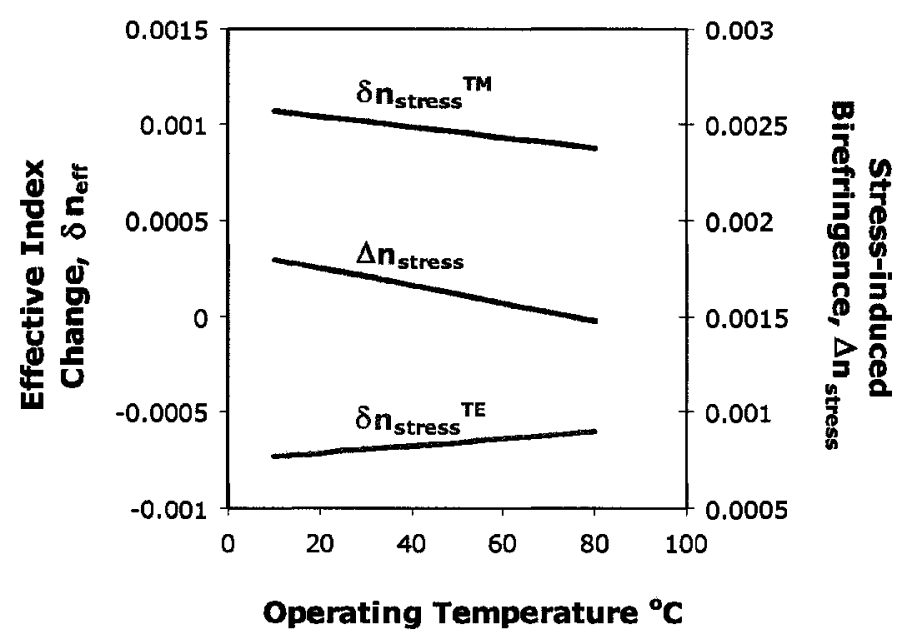

(b)

Figure 4.13: Temperature sensitivity of (a) stress in the upper cladding film $\sigma_{\text {film }}$ (cooling from 400 to $20^{\circ} \mathrm{C}$ ); (b) the stress-induced effective index change and stress-induced birefringence $\Delta n_{\text {stress. }}$. The operating temperature is assumed to vary from $10^{\circ} \mathrm{C}$ to $80^{\circ} \mathrm{C}$. The waveguide has the following specifications: $W=1.5 \mu \mathrm{m}, D=1.4 \mu \mathrm{m}, H=2.2 \mu \mathrm{m}, t=1 \mu \mathrm{m}$, and the operating wavelength is $\lambda_{0}=1550 \mathrm{~nm}$. 


\section{Dispersion}

Dispersion describes the variation of phase velocity with wavelength in a waveguide, leading to signal distortion. There are generally two sources of dispersion: chromatic and intermodal dispersion. Chromatic dispersion occurs in all types of waveguides. It includes material dispersion, which comes from a wavelength-dependent index of a material, and waveguide dispersion, which occurs when the speed of a wave or its effective index in a waveguide depends on its operating wavelength. Intermodal dispersion exists only in multimode waveguides, where each waveguide mode travels at a different speed. By curve-fitting the experimental data quoted from Landolt-Bornstein [84], the material index profiles of the silicon and silica can be approximated by two polynomial functions:

$$
\begin{gathered}
n_{0}(\mathrm{Si})=\left(-2.950 \times 10^{16}\right) \lambda_{0}^{3}+\left(2.244 \times 10^{11}\right) \lambda_{0}^{2}-\left(5.7500 \times 10^{5}\right) \lambda_{0}+3.938 \\
n_{0}\left(\mathrm{SiO}_{2}\right)=\left(-1.70 \times 10^{9}\right) \lambda_{0}^{2}-\left(7.00 \times 10^{3}\right) \lambda_{0}+1.459
\end{gathered}
$$

After incorporating the material dispersion in our numerical model discussed in Chapter 3 , the effective indices of the two orthogonal polarizations are evaluated with and without stress. Fig. 4.14 illustrates the wavelength dependence of the effective indices of the TE and TM modes, by comparing the effects without stress to the case with a compressive stress of $\sigma_{\text {film }}=-330 \mathrm{MPa}$. It can be observed that both effective indices of TE and TM without stress ( $n_{\text {eff }}^{\mathrm{TE}}$ and $n_{\text {eff }}^{\mathrm{TM}}$ ) are almost linearly proportional to wavelength, with a negative slope of $-0.019 / 100 \mathrm{~nm}$ or within a variation of $0.5 \%$ per $100 \mathrm{~nm}$ change in the operating wavelength. Since stress is not included in the calculations of $n_{\text {eff }}^{\mathrm{TE}}$ and $n_{\text {eff }}^{\mathrm{TM}}$, we can conclude that geometry induced effects are reasonably insensitive to operating wavelength. On the other hand, the stress-induced index changes in two polarizations, $\delta n_{\text {stress }}^{\mathrm{TE}}$ and $\delta n_{\text {stress }}^{\mathrm{TM}}$, have slopes of $1.4 \times 10^{-5} / 100 \mathrm{~nm}$ and $-2.5 \times 10^{-8} / 100 \mathrm{~nm}$, respectively. The corresponding variation in the stress-induced index is less than $0.4 \% / 100 \mathrm{~nm}$ for the TM mode while there is virtually no change in $\delta n_{\text {stress }}^{\mathrm{TE}}$. It should be pointed out that the simulations assume a weak wavelength dependence in the stress-optic tensor elements 


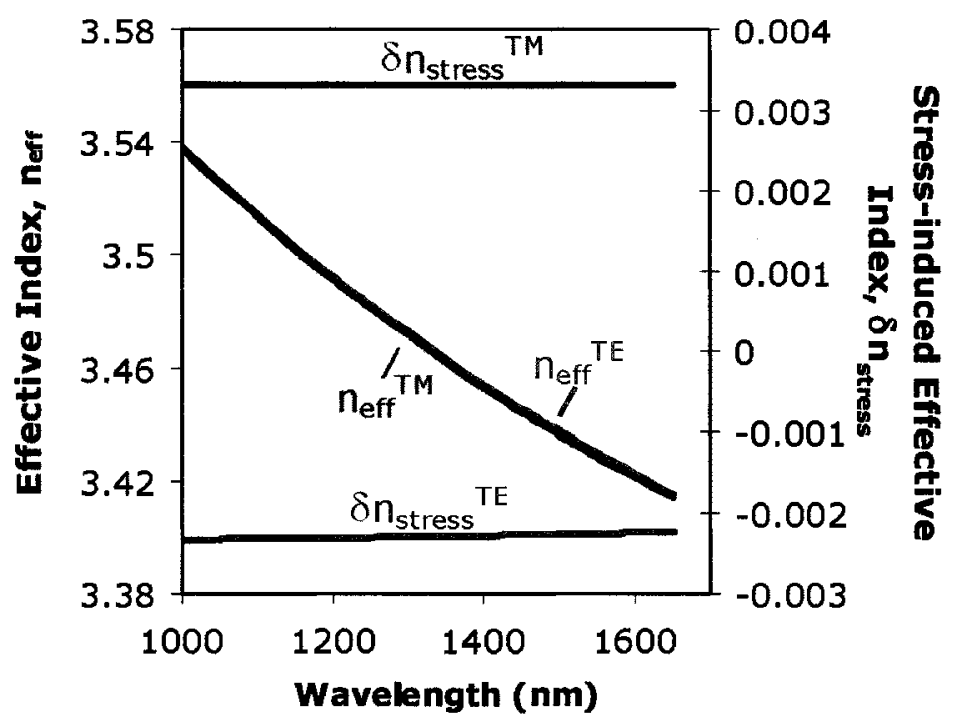

Figure 4.14: The wavelength dependency on the effective index of the TE and TM (i) with no stress $\left(n_{\text {eff }}^{\mathrm{TE}}\right.$ and $n_{\text {eff }}^{\mathrm{TM}}$ ); (ii) with $\sigma_{\text {film }}=-330 \mathrm{MPa}$. The waveguide has the following dimensions: $W=1.5 \mu \mathrm{m}, D=1.4 \mu \mathrm{m}$, $H=2.2 \mu \mathrm{m}$, and $t=1 \mu \mathrm{m}$.

$p_{i j}$ (see Chapter 3 for explanation). Since the mechanism behind the stress-induced polarization compensation and splitting relies solely on the parameter $\delta n_{\text {stress }}$, the stress induced contribution to birefringence will be almost wavelength independent over any reasonable operating range.

\subsubsection{Effects of Ridge Etch Depth with Stress}

The influence of ridge etch depth on geometrical birefringence (without stress) has been described in Fig. 4.5. For the identical rectangular and trapezoidal waveguide geometries, the effects of stress on birefringence with varying etch depths are shown in Fig. 4.15. The compressive stress in the upper oxide cladding $\sigma_{\text {film }}$ is assumed to be $-300 \mathrm{MPa}$. Here the stress-induced birefringence $\left(\Delta n_{\text {stress }}\right)$ is calculated as the difference between the total waveguide birefringence and the geometrical birefringence (shown in Fig. 4.5): $\Delta n_{\text {stress }}=\Delta n_{\text {total }}-\Delta n_{\text {geo }}$.

For shallow etched ridge waveguides, stress does not have significant influence in the 


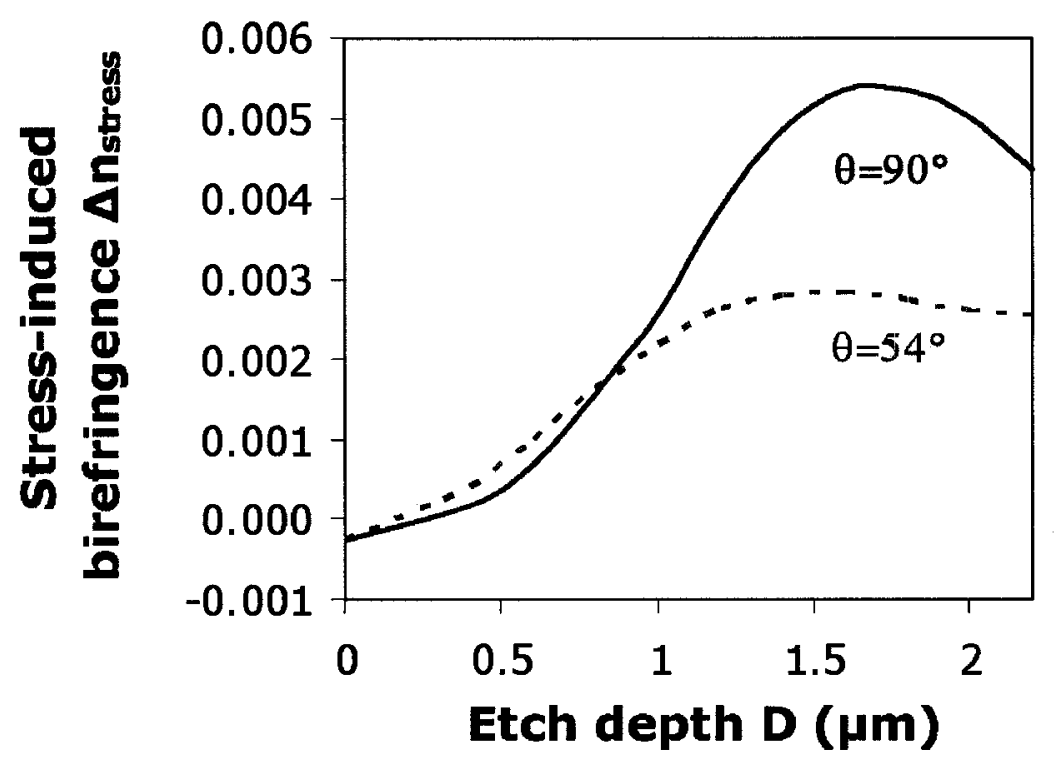

Figure 4.15: Etch depth influence on stress-induced birefringence $\left(\Delta n_{\text {stress }}\right)$ for a rectangular $\left(\theta=54^{\circ}\right)$ and a trapezoidal $\left(\theta=90^{\circ}\right)$ ridge waveguide. The $0.7 \mu \mathrm{m}$ upper oxide cladding film is under a compressive stress of $-300 \mathrm{MPa}$. The waveguide geometry is identical to that defined in Fig. 4.5: $W_{1}=1.5 \mu \mathrm{m}$ and $H=2.2 \mu \mathrm{m}$.

total birefringence. The geometrical contribution, on the other hand, dominates the total birefringence experienced by the waveguides with small etch depths. It is noted that at the etch depth $D=0$ (i.e., a slab waveguide), the birefringence induced by stress is slightly negative (in both cases, $\Delta n_{\text {stress }}=-2.5 \times 10^{-4}$ ). The small negative birefringence is a result of a slight wafer bending which affects the in-plane components $\left(E_{x}\right)$ more than the out-of-plane components $\left(E_{y}\right)$.

Similar to the geometrical birefringence, the stress-induced birefringence is observed to be less sensitive to the fluctuations in etch depth for deeply etched ridge waveguides. For a core thickness of $2.2 \mu \mathrm{m}$, the relative contribution of the stress-induced effects on total birefringence are the strongest at $D=1.5 \mu \mathrm{m}$. This is due to the perfect alignment between the maxima of the electric field (center of the waveguide mode) and the peak strength of the stress field in the core. As the etch depth decreases beyond $1.5 \mu \mathrm{m}$, the stress-induced birefringence is slightly reduced because of the misalignment between the maxima of the modes and the peak strength of the stress contribution. 


\subsubsection{Effects of Ridge Width with Stress}

The geometrical effects of the ridge width without the stress factor have been studied in Fig. 4.6. With the inclusion of the stress-induced effects for the identical waveguide geometries described in Fig. 4.6, we compare the effective indices of TE polarization (with and without stress) for ridge sidewall slopes of $54^{\circ}$ and $90^{\circ}$ in Fig. 4.16(a). The oxide cladding thickness is assumed to be $0.7 \mu \mathrm{m}$ with a stress of $-300 \mathrm{MPa}$. The etch depth is fixed at $1.5 \mu \mathrm{m}$, and the core thickness is $2.2 \mu \mathrm{m}$. The effective indices of the TE modes are reduced under the influence of stress for both the trapezoidal and rectangular ridge waveguides, resulting in birefringence changes. For fluctuations of $\pm 0.1 \mu \mathrm{m}$ in the top ridge width of $1.5 \mu \mathrm{m}$, the change for the dry-etched waveguide (90 sidewall) in the effective index and in the birefringence are $8.4 \times 10^{-3}$ and $1.2 \times 10^{-3}$, respectively. In contrast, the corresponding changes for the wet-etched waveguide (54 sidewall) in $n_{\text {eff }}^{\mathrm{TE}}$ and birefringence $\Delta n_{\mathrm{eff}}$ are $1.5 \times 10^{-3}$ and $7.0 \times 10^{-5}$, respectively. Thus, the ridge waveguides with a trapezoidal cross-section are more tolerant to errors in waveguide dimensions.

Fig. 4.16(b) shows both the geometrical birefringence (dashed curves) and the total birefringence including stress (solid curves) as a function of the ridge width. The birefringence is less sensitive to the ridge width fluctuations for trapezoidal ridge waveguides compared with rectangular waveguides, but trapezoidal ridge waveguides suffer a larger negative geometrical birefringence. As shown in Fig. 4.16(b) a compressive stress in the upper cladding produces a positive offset in the birefringence profile. With the birefringence offset provided by the stress, the zero birefringence condition can now be satisfied even for trapezoidal ridge waveguides. This figure emphasizes that stress provides an additional parameter in keeping the waveguides free of birefringence.

To summarize the effects of ridge etch depth and width, combined with the stressinduced effects, Fig. 4.17 plots the stress-induced birefringence $\Delta n_{\text {stress }}$ as a function of the cross-section aspect ratio $W / H$ and the etch ratio $r$. The oxide cladding is assumed 


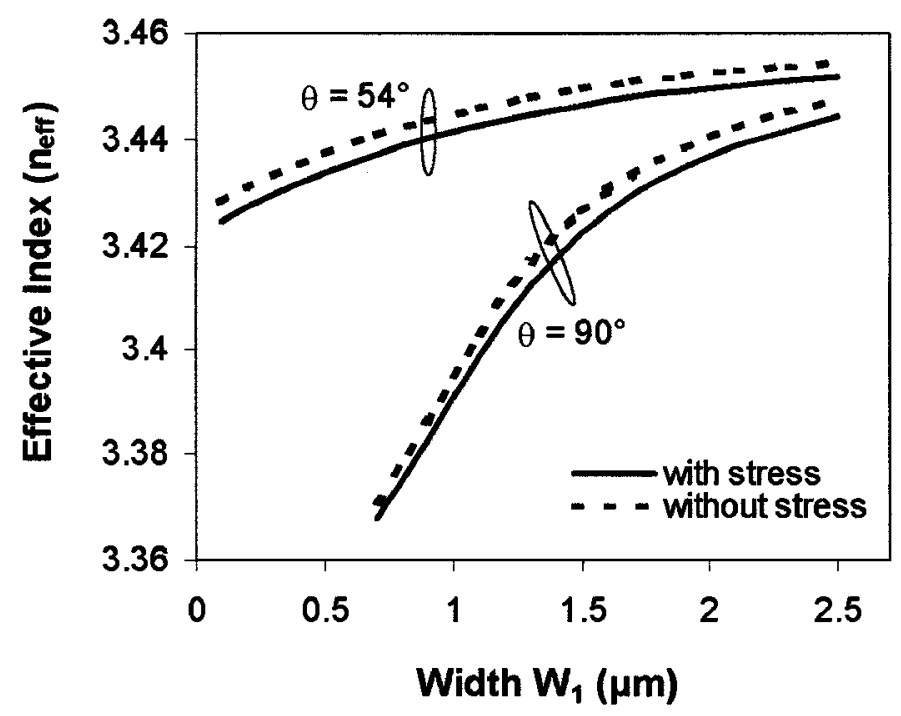

(a)

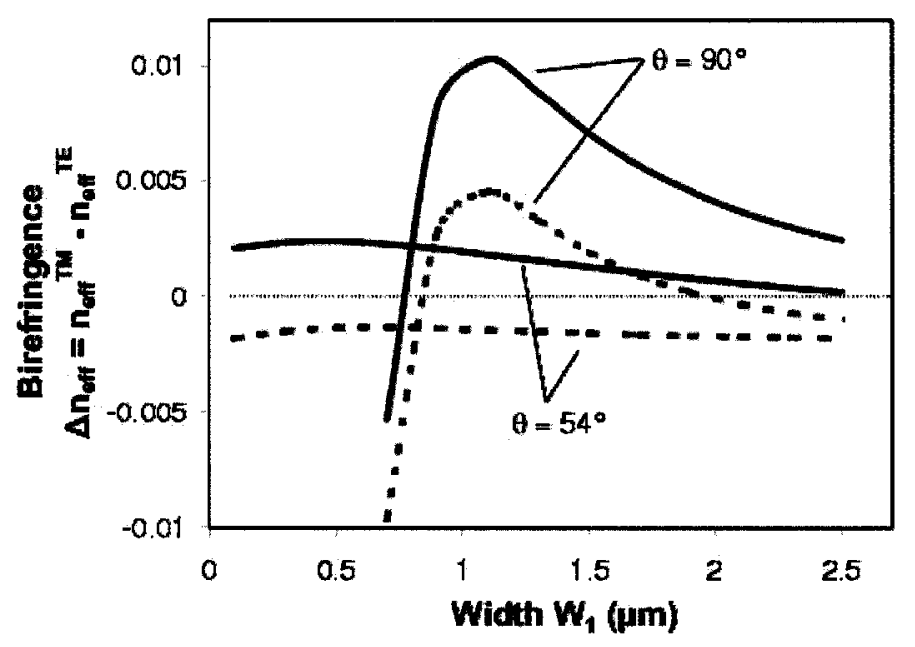

(b)

Figure 4.16: Ridge width influence on waveguide effective index and birefringence for trapezoidal and rectangular waveguides. Shown are (a) effective mode indices of TE polarization with stress (solid curves) and without stress (dashed curves) in trapezoidal $\left(\theta=54^{\circ}\right)$ and rectangular $\left(\theta=90^{\circ}\right)$ ridge waveguides and (b) geometrical birefringence (dashed curves) and total birefringence $\left(\Delta n_{\text {stress }}+\Delta n_{\text {geo }}\right)$ under a compressive stress of $\sigma_{\text {film }}=-300$ MPa (solid curves). The oxide cladding thickness is $t=0.7 \mu \mathrm{m}$. Identical waveguide geometries as in Fig. 4.6 are used: $D=1.5 \mu \mathrm{m}, H=2.2 \mu \mathrm{m}$.

to have a compressive stress of $\sigma_{\text {film }}=-100 \mathrm{MPa}$. Similar to Fig. $4.8, H$ is fixed at $2 \mu \mathrm{m}$ for all the cases. The stress-induced birefringence $\Delta n_{\text {stress }}$ in a rectangular ridge waveguide 


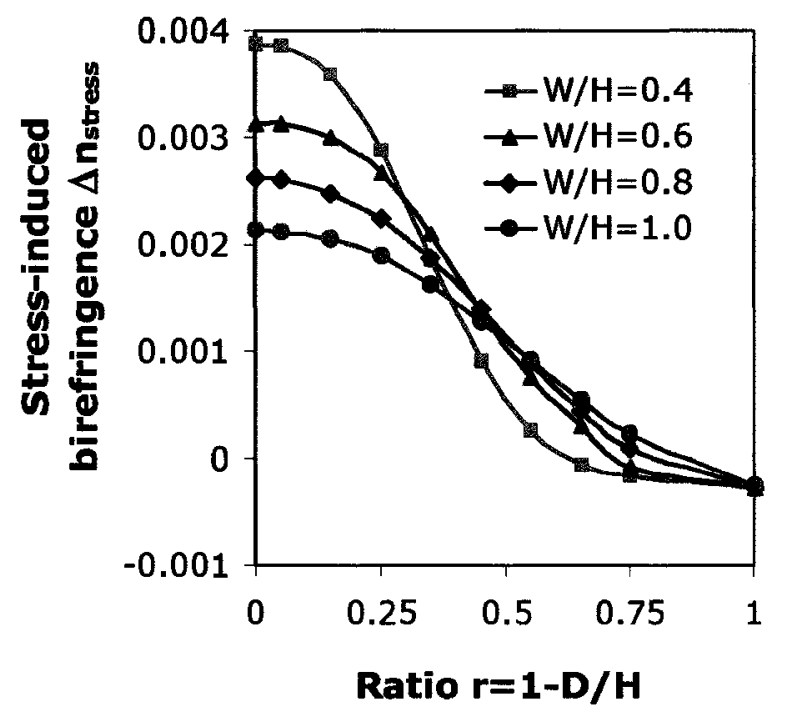

Figure 4.17: Stress-induced birefringence $\Delta n_{\text {stress }}$ as a function of the etch ratio $r=1-D / H$ and the cross-section aspect ratio $W / H$ in rectangular ridge waveguides. Here the silicon core thickness $H$ is fixed at $2 \mu \mathrm{m}$. The oxide cladding is assumed to be $1.5 \mu \mathrm{m}$ thick and a compressive stress of $-100 \mathrm{MPa}$ is assumed.

shows a strong dependence on the ridge etch ratio $r$ where $r=1-D / H$. It is noted that at $r=1$ (with no etch), the birefringence induced by stress is slightly negative, which is consistent with the observation in Fig. 4.15. This is due to the weak wafer bending effects. In general, for shallow etches (with large $r$ values), the relative contribution of stress-induced effects is less significant due to the large misalignment between the peak strength of the stress distribution and the maxima of the filed distribution in the modes. The magnitude of the stress in the cladding film itself is also much smaller in waveguides with shallow etch depths. However, for deeply etched ridge waveguides (with small $r$ ), the change in total birefringence can reach $10^{-3}$, using a cladding film with a film stress of $\sigma_{\text {film }}=-100 \mathrm{MPa}$. It can be also observed from Fig. 4.17 that stressinduced effects are more pronounced for narrower waveguides. Thus stress-induced effects becomes significant as the cross-section aspect ratio and the etch depth increase. 


\subsubsection{Effects of Ridge Height with Stress}

So far only the characteristics of stress-induced effects for waveguides with a constant ridge height have been examined. This section investigates the stress-induced birefringence $\left(\Delta n_{\text {stress }}\right)$ for waveguides with an identical geometry profile but different core sizes (i.e., silicon layer thickness $H$ ). Fig. 4.9 in Chapter 4.1 .3 presented the geometrical birefringence for waveguides with different ridge height $H$. Fig. 4.18 compares the dependence of the stress-induced birefringence on the oxide thickness for rectangular waveguides of varying ridge heights of $1,2,3,4$, and $5 \mu \mathrm{m}$, respectively. The stress in the upper oxide

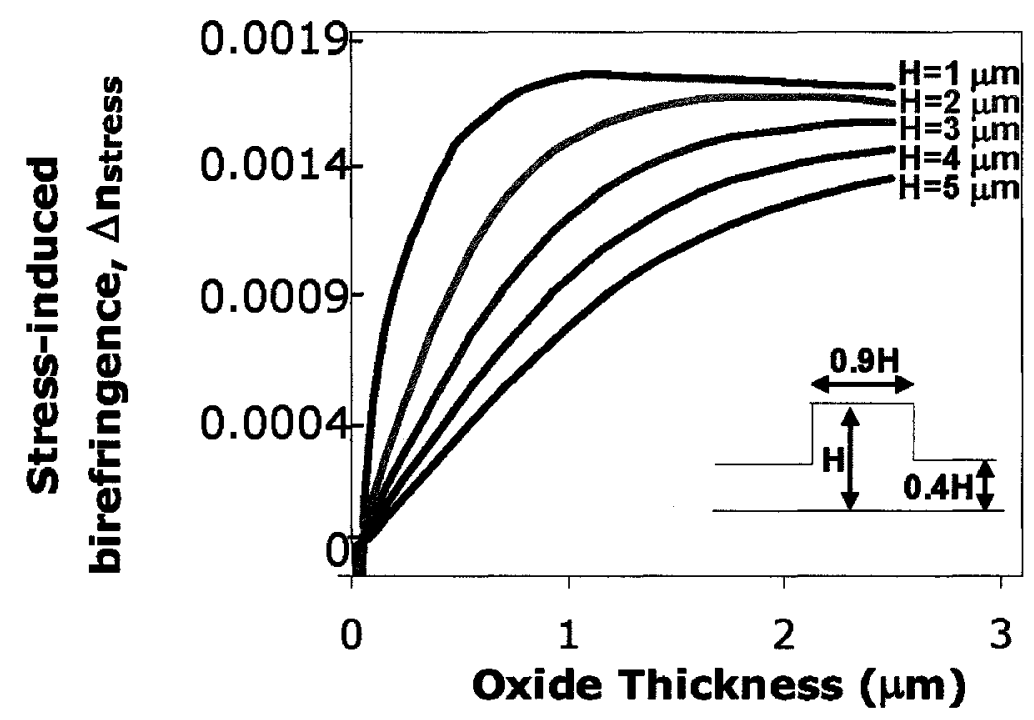

Figure 4.18: Stress-induced birefringence $\Delta n_{\text {stress }}$ (waveguide geometry shown inset) as a function of the the oxide thickness. The stress level in the oxide cladding is assumed to be $\sigma_{\text {film }}=-100 \mathrm{MPa}$.

cladding is chosen to be $-100 \mathrm{MPa}$ in the simulations. For the same cladding thickness, smaller stress-induced effects are observed in ridge waveguides with larger ridge heights. The stress-induced birefringence of a ridge waveguide with fixed dimensions increases as the oxide thickness increases. As the oxide thickness increases further beyond the ridge etch depth, $\Delta n_{\text {stress }}$ saturates and approaches to a maximum level. The saturation values for all the ridge heights considered here are expected to be similar. In the example given here, a maximum of the stress-induced birefringence of $+1.7 \times 10^{-3}$ can be achieved for 
$\sigma_{\text {film }}=-100 \mathrm{MPa}$. The stress-induced effect $\Delta n_{\text {stress }}$ can be much higher if a higher stressed oxide cladding is used.

\subsubsection{Effects of Stress in Photonic Wire Waveguides}

The previous sections focused on the ridge SOI waveguides. As mentioned in Chapter 2, keeping birefringence low is also important for designing and making photonic strip or wire waveguides. The single mode condition depends on both the waveguide height $H$ and the width $W$. The single-mode operation is usually obtained by designing waveguides with square cross-sections. An extreme compact dimension smaller than $0.35 \times 0.35 \mu \mathrm{m}^{2}$ is required for the single-mode propagation at the telecommunication wavelengths. The TE and TM modes exhibit rather different behavior since the modes are strongly dependent on the waveguide geometry. The guiding properties are extremely sensitive to the dimension changes. Fig. 4.19 shows the model birefringence $\Delta n_{\mathrm{eff}}$ as a function of the waveguide width $W$ for a given waveguide height $H=0.5 \mu \mathrm{m}$. In the absence of

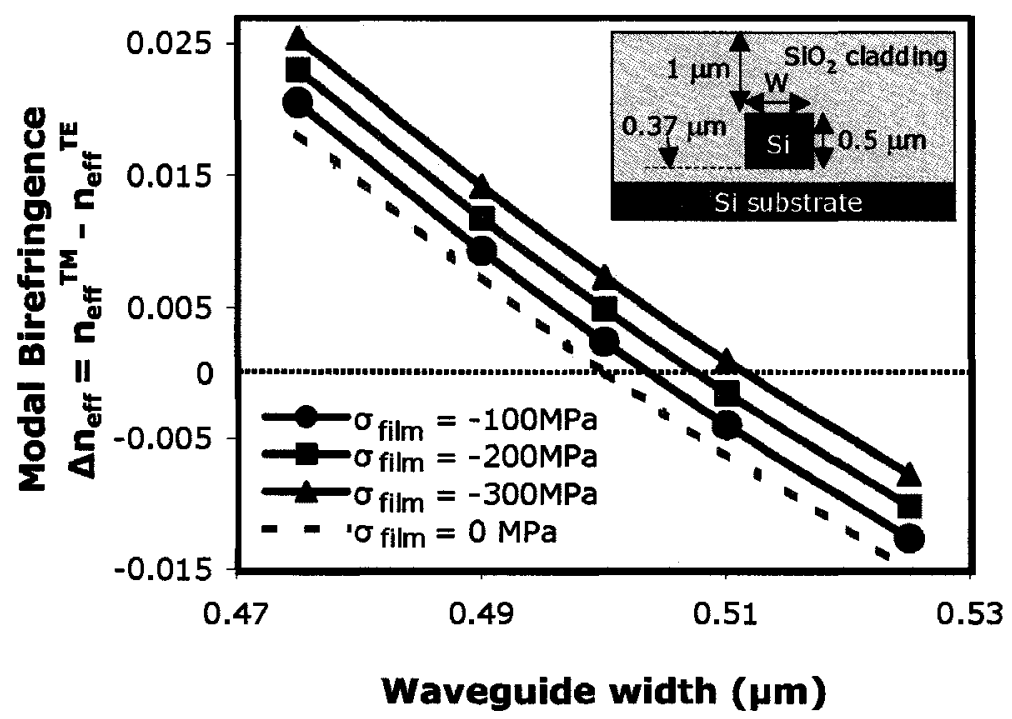

Figure 4.19: The variation of modal birefringence with waveguide width for a photonic wire with dimensions shown in the inset.

stress, the waveguide birefringence is zero for $W=0.5 \mu \mathrm{m}$ (i.e., a square waveguide cross-section), but it is very sensitive to the variations in wire width. It is shown that a 
variation as small as $2 \%$ in $W$ (which corresponds to a change of $10 \mathrm{~nm}$ ) could yeild a birefringence change of $\sim 7 \times 10^{-3}$. Fig. 4.19 suggests that the cladding stress is useful in modifying birefringence in photonic wires. A positive birefringence shift on the order of $10^{-3}$ is achieved by a compressive cladding stress level of $-100 \mathrm{MPa}$. However, the total birefringence is dominated by the waveguide geometry rather than the cladding stress. The fabrication tolerances for making birefringence-free submicron wires are extremely narrow. Although not as effective as for waveguide with core sizes of $1-2 \mu \mathrm{m}$, the stress engineering can still provide some degrees of freedom in birefringence tuning for submicron-sized devices.

\subsubsection{Summary}

The stress-induced birefringence, together with the geometrical effects, form the basis of a new approach to polarization management in SOI ridge waveguides. This chapter examined the influence of the ridge cross-section, aspect ratio, etch depth, and core thickness in the presence of cladding-induced stress. It has been predicted that stress can easily induce a birefringence change on the order of $10^{-3}$ using a stressed oxide film with the nominal film stress in the range of $\sigma_{\text {film }}=-100$ to $-300 \mathrm{MPa}$. This is a significant effect on birefringence. We have found that waveguides can be designed with dimensions in the regime where the geometrical birefringence is appreciable but less sensitive to dimension fluctuations, and that the birefringence-free waveguides can be achieved through stress compensation. The inclusion of stress in the waveguide design enables the decoupling of the cross-section geometry from the birefringence constraints. This property allows the geometry to accommodate other design criteria such as mode confinement, bend loss, minimum allowed bend radius, and coupling between adjacent waveguides, thus optimizing the device performance. In addition, stress induced contribution to birefringence is almost wavelength independent over any reasonable operating range. It is also worth pointing out that an upper cladding has a negligible effect on mode shape, causing almost 
no concern for mode mismatch and the polarization dependent losses associated with the junctions between the clad and unclad waveguides [15]. As described in this chapter, stress engineering can be applied to waveguides of arbitrary cross-section geometry and dimensions for an effective correction of the overall waveguide birefringence. We can conclude that stress engineering is a promising powerful polarization control method. 


\section{Chapter 5}

\section{Experimental Demonstration of}

\section{Stress Engineering}

This chapter presents an experimental demonstration of the modal birefringence control in $\mathrm{SOI}$ waveguides using $\mathrm{SiO}_{2}$ cladding induced stress. We demonstrate the effective birefringence tuning using the stress induced by the upper oxide cladding with different thicknesses in arrayed waveguide grating (AWG) devices. A polarization insensitive AWG device is realized based on stress engineering. All the test AWG devices were fabricated in the Nanofabrication Group at the National Research Council of Canada. The numerical results generated by the implemented simulation models described in Chapter 3 are compared to the experimental measurements obtained by Ms. M.-J. Picard and Dr. D.-X. Xu.

\subsection{Test Arrayed Waveguide Grating Devices}

The AWG is one of the most important integrated optical devices that has been utilized in multiplexing and demultiplexing of wavelengths, switching, add/drop filtering, and polarization splitting. Chapter 2 reviewed some of the recent advances in the AWG applications. A typical AWG consists of input/output waveguides, two focusing slab 
regions (the free propagation regions or FPR's), and an array of waveguides in between the two FPR's. A layout of the AWG demultiplexers is shown in Fig. 5.1. The input

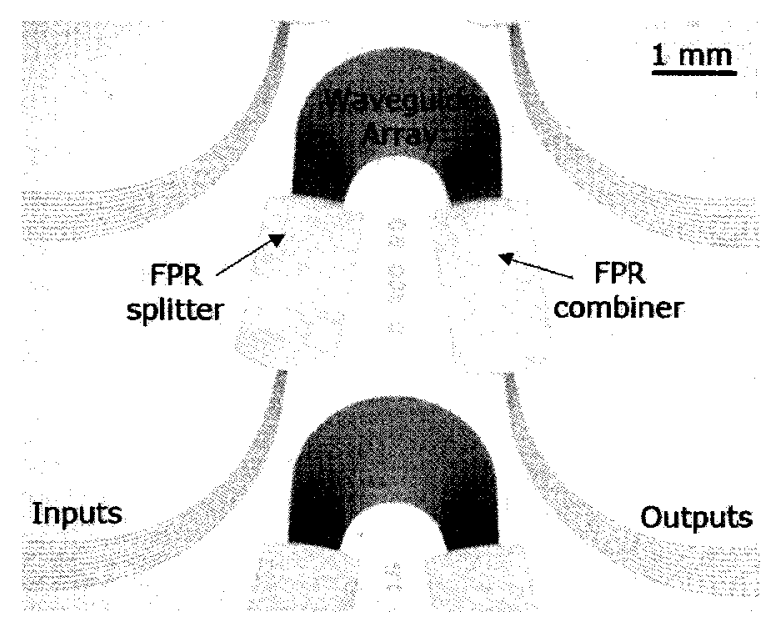

Figure 5.1: A top view of a fabricated SOI AWG demultiplexer.

light is coupled into a single input waveguide leading to the FPR splitter region where it diffracts in the slab waveguide and couple into an array of waveguides. Each waveguide in the arrayed section is longer than the adjacent waveguide by a constant length increment $\Delta L=M \lambda_{0}$, where $M$ is an integer and $\lambda_{0}$ is the center operating wavelength. The arrayed waveguides converge at the second FPR combiner region, where field distributions from the arrayed waveguides are transformed into a convergent beam and focus on the output image plane. Because of the length increment between each array waveguide, the corresponding phase difference between light emerging from adjacent waveguides is $\Delta \phi=2 \pi n_{\mathrm{eff}} \Delta L / \lambda_{0}$ where $n_{\mathrm{eff}}$ is the effective index of the fundamental waveguide mode. At the center wavelength $\lambda_{0}$ this phase shift is an integer multiples of $2 \pi$, the output is a perfect mirrored image of the input. As the wavelength increases or decreases, the phase change is no loner an exact multiple of $2 \pi$, and the convergent beam is focused away from the center output waveguide. In a wavelength demultiplexer, birefringence results in a wavelength shift in the spectral response peaks of the TE and TM polarization of each wavelength channel, which limits the wavelength resolution and affects the spectral channel density. In this chapter, we discuss the use of stress engineering to control and 
eliminate birefringence in the AWG demultiplexer.

Test AWG devices (shown in Fig. 5.1) were fabricated on SOI $<100>$ wafers, with a 2.2 $\mu \mathrm{m}$ thick Si core layer and a $0.37 \mu \mathrm{m}$ thick buried oxide layer. The devices were designed to operate at a central wavelength of $\lambda=1550 \mathrm{~nm}$. The waveguide arrays consisted of 100 ridge waveguides with a length increment between two adjacent waveguides of $22.68 \mu \mathrm{m}$, corresponding to a grating order of $m=n_{\mathrm{eff}} \frac{\Delta L}{\lambda_{0}}=49$. The fabricated devices were made with 9 channels. The output channel waveguides were separated by $8.8 \mu \mathrm{m}$ at the combiner focus, to give a $200 \mathrm{GHz}$ (or $1.6 \mathrm{~nm}$ ) channel spacing. The overall chip size was approximately $5 \mathrm{~mm} \times 5 \mathrm{~mm}$. More details of the design can be found in [31].

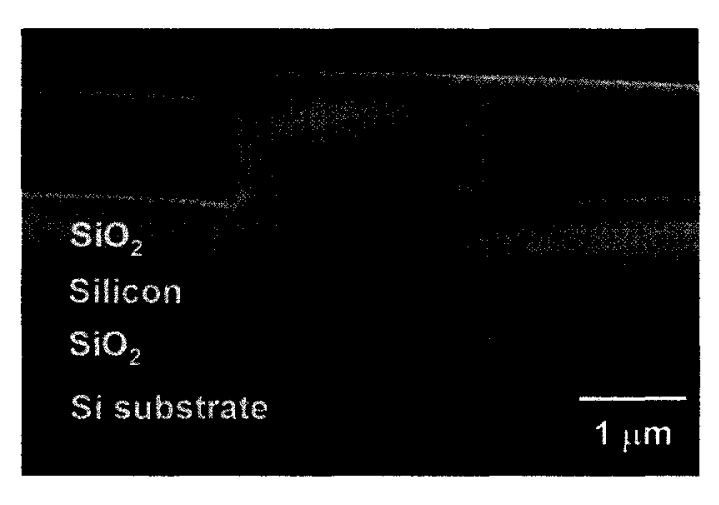

(a)

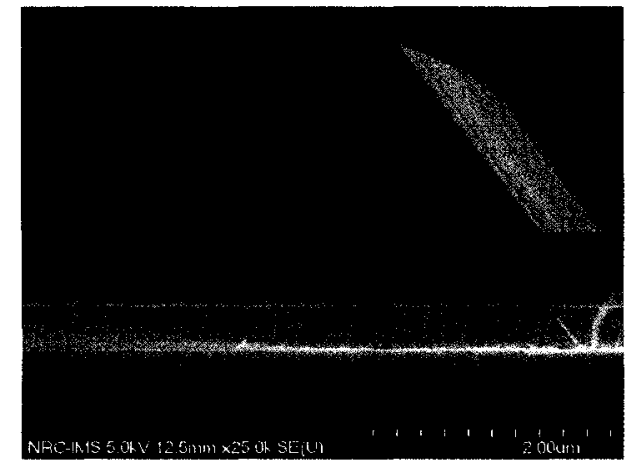

(b)

Figure 5.2: SEM images of the cross section of a ridge waveguide in the arrayed grating section (see Fig. 5.1). Shown are (a) a dry-etched waveguide with a PECVD deposited oxide cladding (same as Fig. 4.1) and (b) a wetetched waveguide without cladding deposition.

The waveguides in the devices have nominal ridge widths of 1.5 and $2 \mu \mathrm{m}$. They were fabricated using both dry and anisotropic wet etch methods, yielding a nearly vertical ridge sidewall angle of $87^{\circ}$ and a slanted sidewall slope of $54^{\circ}$, respectively. The actual ridge dimensions for the waveguides were measured from scanning electron microscope (SEM) images taken at several locations along the waveguide bends in the arrayed grating section. Fig. 5.2 shows SEM images of typical waveguide cross-sections fabricated by ICP RIE (inductively coupled plasma reactive ion etching) and by wet-etch methods. The measured data for the fabricated ridge dimensions are listed in Table 5.1. 
The variations in the measured ridge widths were within $5 \%$ of the average for the dryetched waveguides. The wet-etched ridge waveguides, on the other hand, had variations in the width measurements of up to $\pm 20 \%$, depending on the angle of the waveguide propagation axis relative to the major crystal planes.

\begin{tabular}{|c|c|c|c|}
\hline Etch Type & Ridge Width $W_{1}$ & Ridge Width $W_{2}$ & Etch Depth $D$ \\
\hline \hline Dry Etch $(1.5 \mu \mathrm{m}$ nominal width $)$ & $1.35 \mu \mathrm{m} \pm 4 \%$ & $1.5 \mu \mathrm{m} \pm 1 \%$ & $1.47 \mu \mathrm{m} \pm 1 \%$ \\
Dry Etch $(2.0 \mu \mathrm{m}$ nominal width $)$ & $1.85 \mu \mathrm{m} \pm 2 \%$ & $2.0 \mu \mathrm{m} \pm 5 \%$ & $1.47 \mu \mathrm{m} \pm 3 \%$ \\
\hline Wet Etch $(1.5 \mu \mathrm{m}$ nominal width) & $1.1 \mu \mathrm{m} \pm 20 \%$ & $3.8 \mu \mathrm{m} \pm 10 \%$ & $1.47 \mu \mathrm{m} \pm 2 \%$ \\
Wet Etch $(2.0 \mu \mathrm{m}$ nominal width $)$ & $1.5 \mu \mathrm{m} \pm 11 \%$ & $4.0 \mu \mathrm{m} \pm 7 \%$ & $1.47 \mu \mathrm{m} \pm 2 \%$ \\
\hline
\end{tabular}

Table 5.1: List of measured ridge dimensions. The targeted etch depth is $1.5 \mu \mathrm{m}$ for both the dry- and wet-etched waveguides.

As mentioned in Chapter 4, the upper oxide cladding thickness $(t)$ is a parameter that can effectively control the stress-induced birefringence. In the experimental demonstration, the upper oxide cladding was deposited by plasma-enhanced chemical vapor deposition (PECVD) at $400{ }^{\circ} \mathrm{C}$. The PECVD processing utilizes a plasma to enhance chemical reaction rates of the source gases and allows deposition at a relatively low temperature (below $400^{\circ} \mathrm{C}$ ) [91]. Compared to the thermal oxidation process where the $\mathrm{SiO}_{2}$ was formed on the silicon surface in an oxygen rich atmosphere, the low temperature deposition was the biggest advantage of PECVD (thermal oxidation is characterized by high deposition temperatures at 900 to $1100^{\circ} \mathrm{C}$ ). In addition, the PECVD has a faster deposition rate than the dry thermal oxidation process. The source gases used in our PECVD deposition included the $\mathrm{SiH}_{4}, \mathrm{~N}_{2}$ and $\mathrm{N}_{2} \mathrm{O}$, and the deposition rate was $\sim 10 \mathrm{~nm} / \mathrm{min}$. The stress in a PECVD grown $\mathrm{SiO}_{2}$ film typically ranges from $\sigma_{\text {film }}=-100$ to -400 MPa. The thickness of the cladding film was controlled by the deposition conditions. The non-uniformity in the thickness of the oxide film produced by the PECVD in our laboratory is normally better than $5 \%$ over the wafer or from run to run. The cladding 
thickness covering the ridge sidewalls was measured to be consistently around $70 \%$ of that on the top of the waveguiding layer $[89,92]$.

The value of the average stress level in a blanket thin film $\left(\sigma_{\text {film }}\right)$ was obtained experimentally by measuring the wafer's curvature radius and using the following relation:

$$
\sigma_{\text {film }}=\frac{E h^{2}}{(1-\nu) 6 R t},
$$

where $E /(1-\nu)$ is the biaxial elastic modulus of the substrate, $h$ is the substrate thickness, $t$ is the film thickness, $R$ is the substrate radius of curvature, and $\sigma_{\text {film }}$ is the average film stress, as shown in Fig. 5.3. The bow radii of the wafer before $\left(R_{1}\right)$ and after $\left(R_{2}\right)$ film deposition were first measured. The effective radius of curvature was then obtained by using

$$
R=\frac{R_{1} R_{2}}{R_{1}-R_{2}}
$$

A compressive stress between -250 to $-320 \mathrm{MPa}$ were measured from our existing samples.

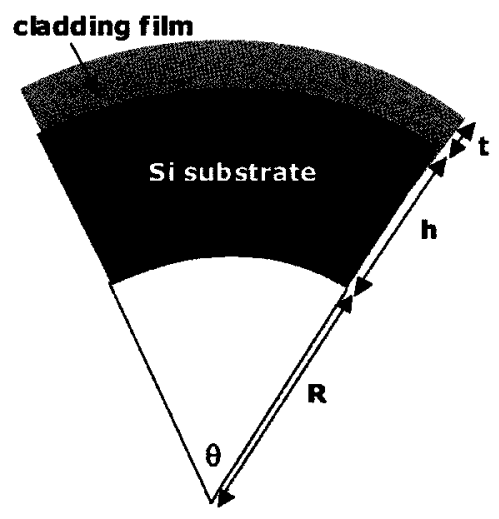

Figure 5.3: A schematic of a bent wafer for stress calculation.

\subsection{Experimental Setup}

Fig. 5.4 shows a schematic of the experimental setup used to measure the spectral response of the test AWG demultiplexer devices. A tunable laser was used to scan the input 
wavelengths. It has a tunable wavelength ranging from $\lambda=1460 \mathrm{~nm}$ to $1580 \mathrm{~nm}$ with a resolution of $0.1 \mathrm{pm}$. The output of the laser was fed through a polarization-maintaining fiber to a in-line polarization rotator such that the input beam can be polarized in either the TE or the TM direction. The beam is then focused onto the input facet of the test device by a single mode fiber with a tapered tip. The size of the fiber tip is $\sim 2-4 \mu \mathrm{m}$ in diameter. Light passes through the device and is then collected by a photodetector through a focusing lens. The power meter that reads out the final output power is installed in the same mainframe as the tunable laser. The spectral response of the demultiplexer test devices could be obtained by taking output power measurements while scanning the wavelength. The camera and the monitor system is used to visualize the optical output beam of the test devices.

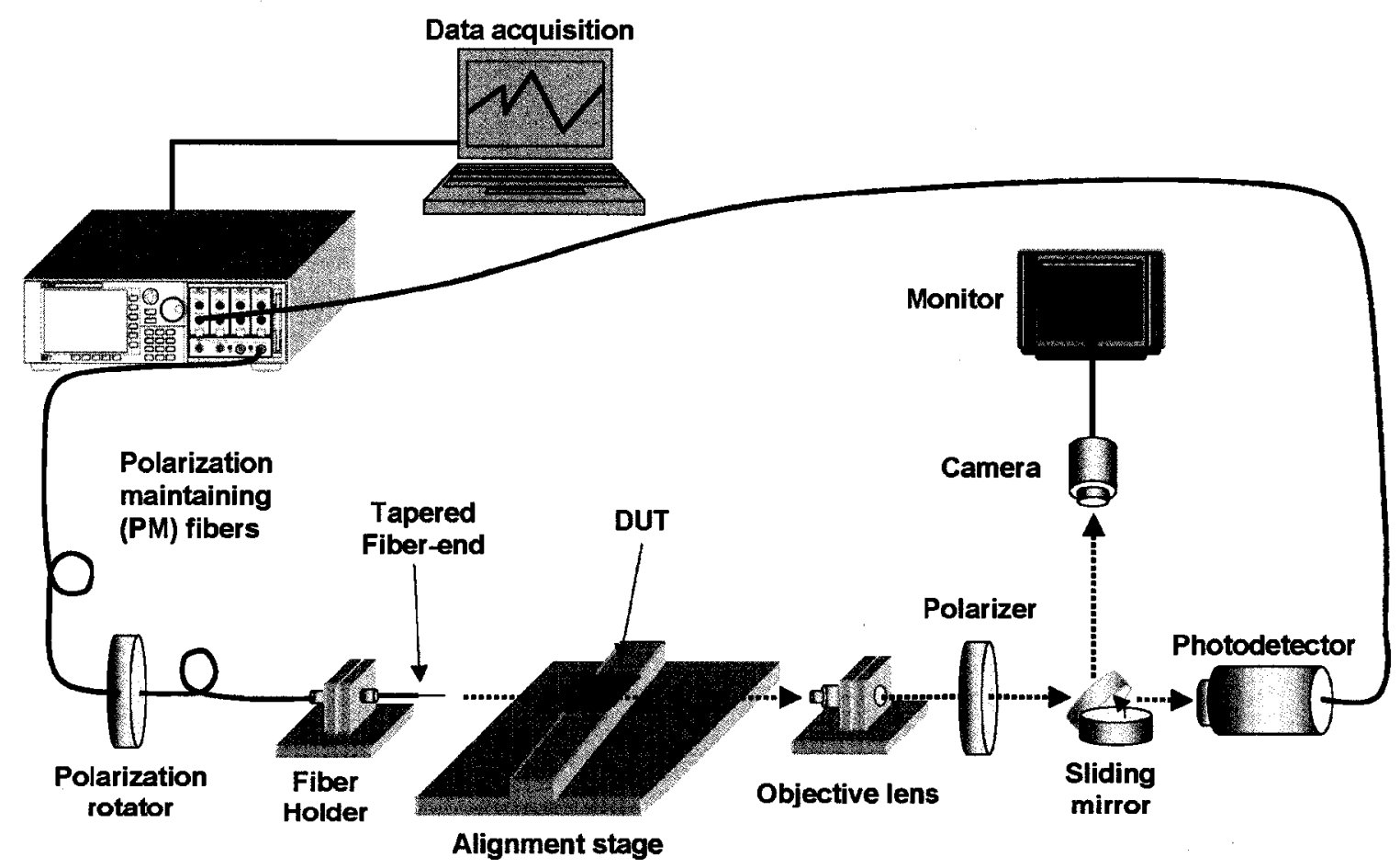

Figure 5.4: A schematic of the experimental setup for waveguide testing. 


\subsection{Results}

\subsubsection{Polarization Tuning Using Stress}

An example of the output spectra of a wet-etched $200 \mathrm{GHz} 9$-channel test AWG demultiplexer is shown in Fig. 5.5. The measurements were performed by Ms. M.-J. Picard and Dr. D.-X. Xu. A crosstalk less than $-20 \mathrm{~dB}$ between adjacent channels and an insertion loss of about $-7 \mathrm{~dB}$ were observed [15]. A polarization dependent wavelength

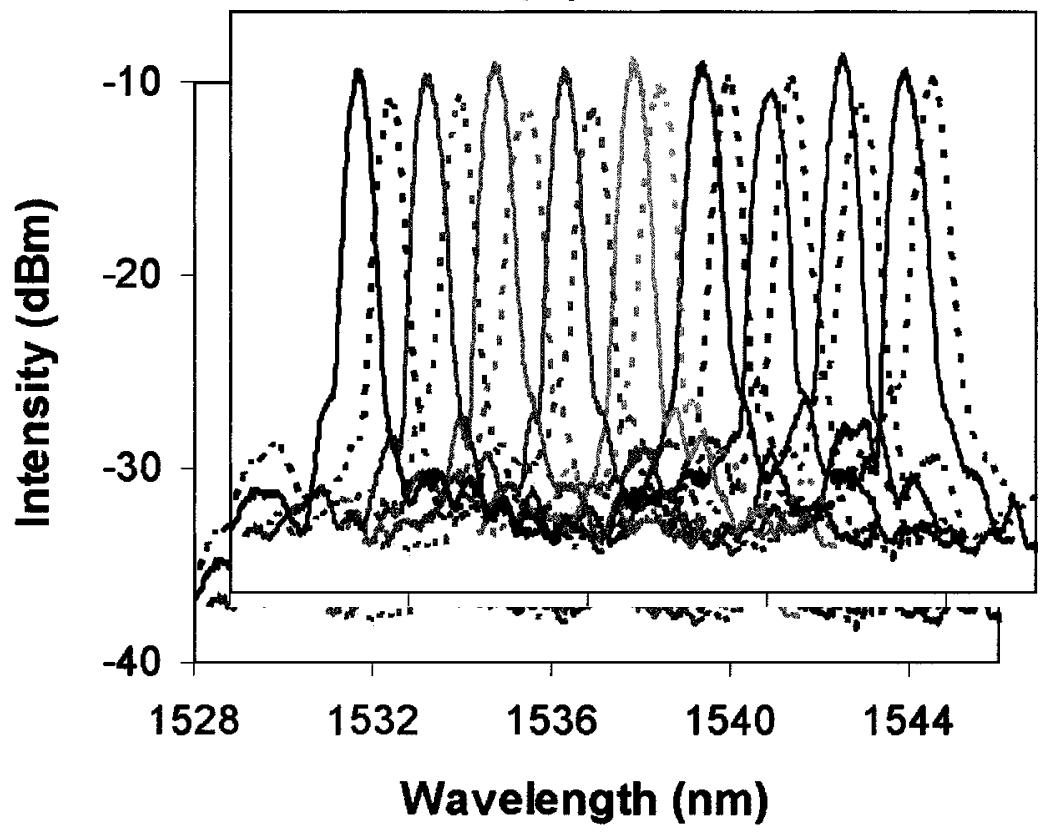

Figure 5.5: Measured output TE (dashed) and TM (solid) spectra of a wet etched $200 \mathrm{GHz}$ 9-channel SOI AWG with ridge height $H=2.2 \mu \mathrm{m}$, ridge widths $W_{1}=1.1 \mu \mathrm{m}$ and $W_{2}=3.8 \mu \mathrm{m}$, and etch depth $D=1.47 \mu \mathrm{m}$. There is no upper oxide cladding.

shift $\Delta \lambda$ between the TE and the TM polarization was observed in the output spectra obtained. Here the wavelength shift $\Delta \lambda$ is defined as the difference between the demultiplexer channel peak wavelengths measured for the TM and TE polarizations; that is, $\Delta \lambda=\lambda_{\text {peak }}^{\mathrm{TM}}-\lambda_{\text {peak }}^{\mathrm{TE}}$. The waveguide birefringence in the arrayed grating section was calculated from the measured $\Delta \lambda$ in the AWG demultiplexer [23]: $\Delta n_{\text {eff }}=n_{\mathrm{g}} \cdot \Delta \lambda / \lambda$, where $n_{\mathrm{g}}=n_{0}-\lambda \frac{\partial n}{\partial \lambda}$ is the group index of the waveguide. In these calculations, $\lambda$ and $n_{\mathrm{g}}$ were assumed to be $1550 \mathrm{~nm}$ and 3.6 , respectively. A wavelength shift of $\Delta \lambda=-0.6 \mathrm{~nm}$ 
was measured from Fig. 5.5, which corresponds to a birefringence level of $-1.3 \times 10^{-3}$.

The upper oxide cladding was then deposited in three separated steps, yielding oxide thickness of $0.3,0.8$, and $1 \mu \mathrm{m}$, respectively. The polarization dependent wavelength shift $\Delta \lambda$ was measured after each deposition step. The compressive stress in the oxide film was measured as $\sigma_{\text {film }}=-320 \pm 5 \mathrm{MPa}$ for both the dry- and wet-etched ridge waveguides. Fig. 5.6 plots the measured wavelength shift as a function of the upper cladding thickness averaged over several AWG channels. The dry-etched ridges have slightly slanted sidewalls, as indicated in Fig. 5.6(a), and the wet-etched ridges have sidewall angles of approximately $54^{\circ}$ (shown in Fig. 5.6(b)). The solid curves shown in Fig. 5.6 were the FEM calculations based on the actual measured ridge dimensions, by using the normalized plane strain model with material parameters listed in Table 3.1.

The expected uncertainty in the calculated birefringence based on the measured device parameters can be estimated using the curves presented in Fig. 4.12 through Fig. 4.16. Fig. 4.16(b) shows that small variations in width for narrow rectangular (dry-etched) waveguides result in larger changes in birefringence. A $5 \%$ uncertainty around $1.5 \mu \mathrm{m}$ in dry-etched ridge widths corresponds to a $15 \%$ uncertainty in birefringence. A $1 \%$ uncertainty around $1.5 \mu \mathrm{m}$ in measured etch depth corresponds to a $5 \%$ uncertainty in birefringence. In contrast, the trapezoidal (wet-etched) and wider (eg, $2.0 \mu \mathrm{m}$ ) rectangular ridge waveguides are relatively insensitive to variations in ridge widths. Similarly, variations in etch depth have almost no effect for trapezoidal and deep etched rectangular ridge waveguides, as suggested in Figs. 4.5 and 4.15. These considerations explain the close agreement between the simulation results and the experimental data for the wider dry-etched waveguides (bottom curve in Fig. 5.6(a)) and the wet-etched waveguides in Fig. 5.6(b).

Overall, there is a good agreement between the simulation results and the experimental data. The scattering in the experimental data is consistent with the small variations in width $\left(W_{1}\right.$ and $\left.W_{2}\right)$, etch depth $(D)$ and stress $\left(\sigma_{\text {film }}\right)$ in the fabricated devices. 


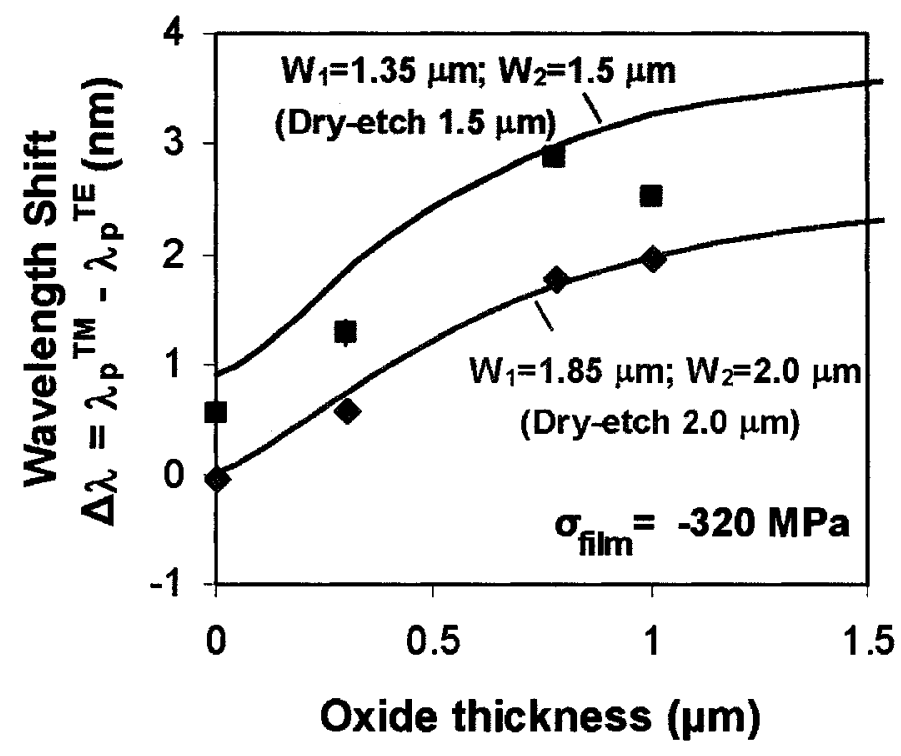

(a)

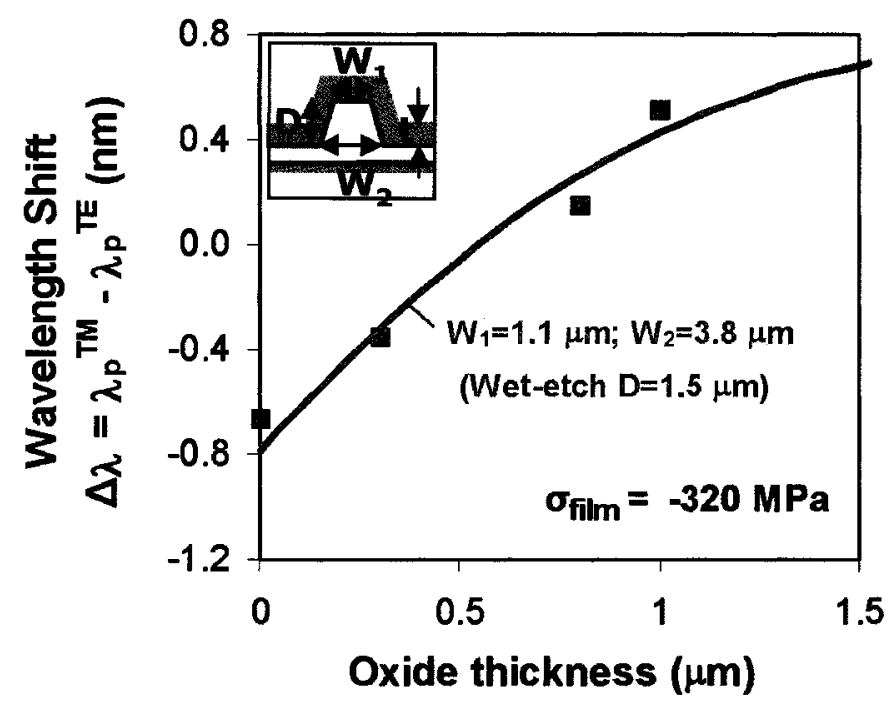

(b)

Figure 5.6: The measured (data points) and calculated (solid curves) wavelength shift $(\Delta \lambda)$ in the demultiplexer's spectra for varying oxide thickness. Shown are (a) dry-etched waveguides and (b) wet-etched waveguides, with stress of $\sigma_{\text {film }}=-320 \mathrm{MPa}$. The ridge geometrical parameters are shown in the inset of (b). The ridge edge depth was measured as $1.47 \mu \mathrm{m}$. Stress was determined from the measurements on wafer bow radius. 


\subsubsection{Polarization-independent Operation Using Stress}

We can conclude from Fig. 5.6 that the birefringence can be tuned with the thickness of the upper oxide cladding. Next a polarization-independent AWG device using this technique is demonstrated. The same test device described in Fig. 5.5 was considered here. When there is no oxide cladding, the measured shift in the spectrum of the AWG was approximately $\Delta \lambda=-0.6 \mathrm{~nm}$, as shown in Fig. 5.5. This polarization dependent

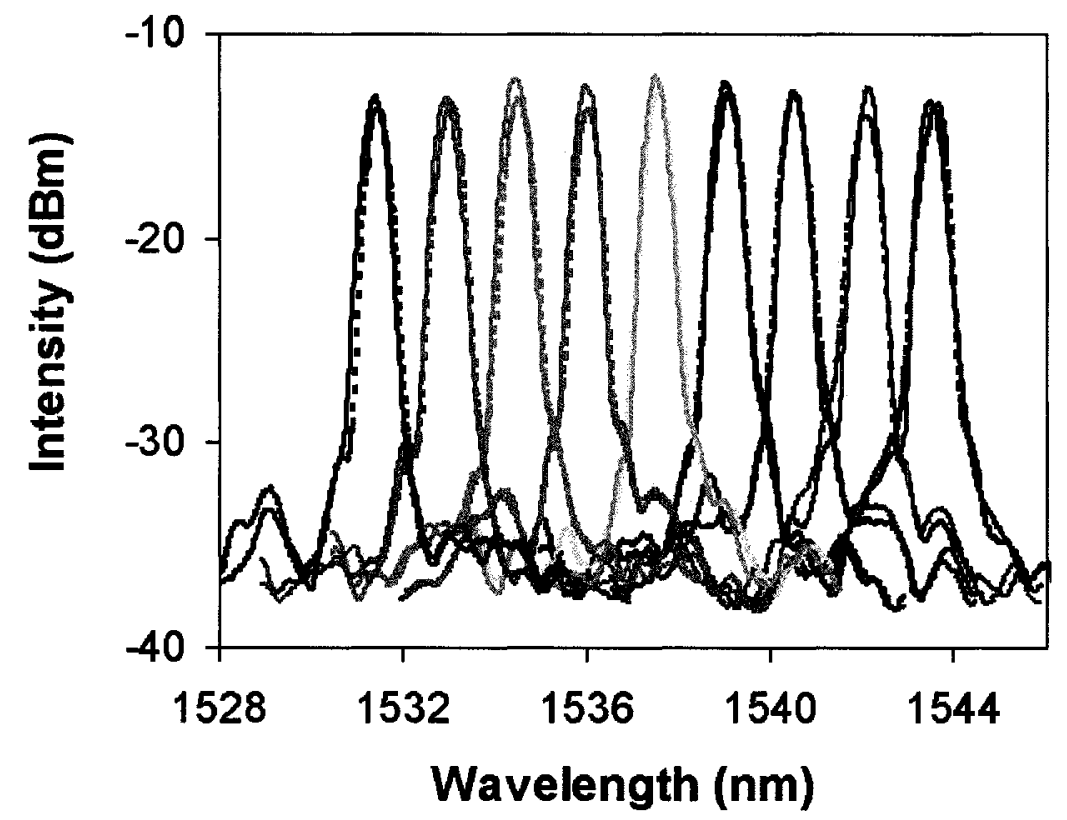

Figure 5.7: The measured output TE (dashed) and TM (solid) spectra of a wet etched $200 \mathrm{GHz}$ 9-channel SOI AWG after stress compensation. The geometry of the ridge waveguides is identical to that defined in Fig. 5.5. An upper oxide cladding thickness of $0.6 \mu \mathrm{m}$ was used to compensate and eliminate the large polarization dependent wavelength shift.

wavelength shift is equivalent to a birefringence level of $-1.3 \times 10^{-3}$, which is unacceptable for most of the AWG demultiplexers. Based on the extrapolated data plotted in Fig. 5.6(b), a $0.6 \mu$ m-thick cladding film is required to eliminate the large negative birefringence. Experimentally, an upper oxide cladding of $0.6 \mu \mathrm{m}$ thickness almost fully compensated the polarization dependent wavelength shift, as shown in Fig. 5.7. A wavelength shift of $\Delta \lambda<-0.02 \mathrm{~nm}$ was observed, which corresponds to a birefringence less than $-4 \times 10^{-5}$. This implies that the waveguides in the phase-array section of the 
polarization compensated AWG are nearly birefringence-free.

The cladding thickness modifies the stress-induced birefringence; however, the stress level in the cladding film remains unchanged. Thermal anneals can be used to alter the stress level in the oxide film, providing another practical method for birefringence tuning. We have demonstrated that a modification in the wavelength shift $\Delta \lambda$ of $0.2 \mathrm{~nm}$ can be produced by annealing at $650^{\circ} \mathrm{C}$ for up to 20 minutes [90].

\subsection{Summary}

It can be concluded from the analysis of the experimental data that the choice of the cladding thickness and the stress level in the oxide cladding are two effective parameters for birefringence tuning. The results presented in this chapter not only validate our predictions of the stress effects on birefringence in Chapter 4, but also confirm the usefulness and practicality of the approach. 


\section{Chapter 6}

\section{Mach-Zehnder Interferometer Based Polarization Splitters/Filters}

In Chapter 1 and 2, we explained the undesirable effects of waveguide birefringence. Two possible solutions to manage birefringence have been discussed: one is the polarization diversity scheme where polarization splitters, filters, and converters are implemented to separate the two orthogonally polarized modes and process them individually; the other is the polarization insensitive option where devices are designed to operate independently of polarization. In this study, we focus on the application of stress engineering to the polarization diversity scheme in realizing stress-induced passive polarization splitters and filters.

The aim of this chapter is to design, fabricate and characterize novel passive polarization splitters/filters based on the Mach-Zehnder interferometer (MZI) configuration. The uniqueness of these devices lies in the use of cladding stress engineering for polarization control. The stress created by the upper oxide cladding layer effectively modifies the overall waveguide birefringence. By choosing an appropriate thickness of the cladding or a film stress level, the birefringence in the waveguide can be precisely controlled. This chapter first describes the operating principles of various configurations of the MZI-based 
polarization splitters/filters. The phase conditions that are required for polarization splitting will be derived separately for each configuration, and the requirements on the upper cladding film will be analyzed and specified. After that, the design choice of the ridge cross-sectional geometry and the $3 \mathrm{~dB}$ couplers will be discussed, followed by the simulation results. An optical mask is then laid out, including a wide range of device geometry variations to accommodate inaccuracies in fabricated device dimensions. Finally, the performance of the fabricated test devices is investigated in detail, and compared with the numerical simulation results.

\subsection{Introduction}

The phase change of an optical mode after propagating a distance $L$ is defined as:

$$
\phi=\frac{2 \pi}{\lambda_{0}} n_{\mathrm{eff}} L
$$

where $n_{\text {eff }}$ is the polarization dependent effective index. The manipulation of the stressinduced phase difference between the TE and TM modes forms the fundamental basis of our proposed polarization splitters.

The relative phase difference between the TE and TM mode must be tuned in order to achieve a polarization splitting function. It is apparent from Eq. 6.1 that there are two tuning parameters: $n_{\text {eff }}$ and length $L$. Based on what have been studied on stress engineering, stress can be used to tune the effective indices of TE and TM, while tailored cladding patches can be applied at discrete locations on top of the ridge waveguides. Thus, the polarization dependent stress-induced phase shift can be written as:

$$
\Delta \phi=\frac{2 \pi}{\lambda_{0}} \delta n_{\text {stress }} L
$$

where $\delta n_{\text {stress }}$ is the stress-induced index change (i.e., $\delta n=n_{\text {eff }}^{\text {with stress }}-n_{\text {eff }}^{\text {no stress }}$ ) and $L$ is the cladding patch length. 
A basic balanced Mach-Zehnder interferometer (MZI) consists of an input and an output $3 \mathrm{~dB}$ coupler, connected by a pair of parallel arms of equal length, as shown in Fig. 6.1. The input and output 3dB couplers can be Y-junctions, multimode interference

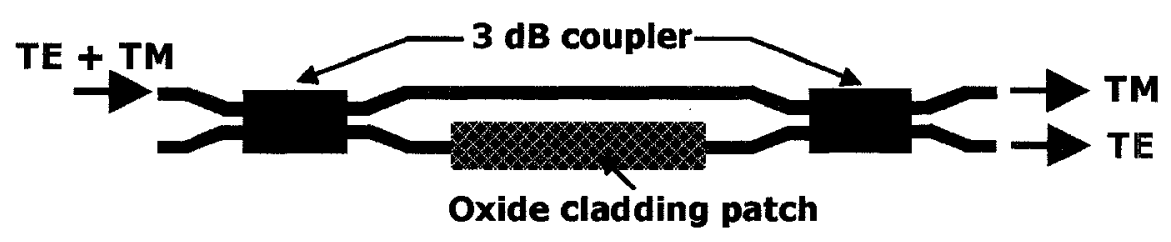

Figure 6.1: Schematic diagram of a basic balanced MZI-based polarization splitter with two $3 \mathrm{~dB}$ couplers. The oxide cladding patch provides the necessary phase shift for polarization splitting.

(MMI) couplers, or directional couplers. Polarization splitting and filtering can be realized by applying a cladding patch on one of the interferometer arms in an MZI design. The stress in the waveguide cladding induces a polarization dependent phase difference between the two light beams propagating in the parallel arms. With a proper choice of the cladding-induced stress, a zero overall relative phase shift (modulo of $2 \pi$ ) between the two arms can be achieved for one polarization ( $\mathrm{TE}$, for example) while a $\pi$ phase shift is induced for the other polarization (TM), resulting a constructive interference for one polarization (TE) and a destructive interference for the other polarization (TM) at one output coupler port. The device can therefore operate as either a polarization splitter or filter, depending on the number of the available output ports of the combiner coupler. Mathematically, the phase relations of a polarization filter or a splitter can be expressed as follows,

A TM Filter or a splitter with TE (bottom port) and TM (top port):

$$
\Delta \phi^{\mathrm{TE}}=2 M \pi \text { and } \Delta \phi^{\mathrm{TM}}=2(N+1) \pi ;
$$

OR

A TE Filter or a splitter with TE (top port) and TM (bottom port):

$$
\Delta \phi^{\mathrm{TE}}=2\left(M^{\prime}+1\right) \pi \text { and } \Delta \phi^{\mathrm{TM}}=2 N^{\prime} \pi,
$$


where $M, N, M^{\prime}, N^{\prime}$ are integers, and the polarization dependent stress-induced $\Delta \phi$ is defined in Eq. 6.2.

\subsection{Splitter/Filter Operating Principles}

The following subsections derive the phase relations and critical design parameters for different types of the MZI-based polarization filtering and splitting devices. The organization of the subsections is as follows. We start with a simple MZI device with two Y-junction couplers. Since the device has a single output port, these devices can only provide a polarization filtering function. By replacing the output Y-junction coupler with a two-port $3 \mathrm{~dB}$ coupler, that is, either a directional coupler or an MMI coupler, the resulting devices can function as polarization splitters. MZI-based polarization splitters with two identical $3 \mathrm{~dB}$ couplers are also investigated, by including symmetric directional couplers or symmetric MMIs.

\subsubsection{MZI with Two Y-junctions}

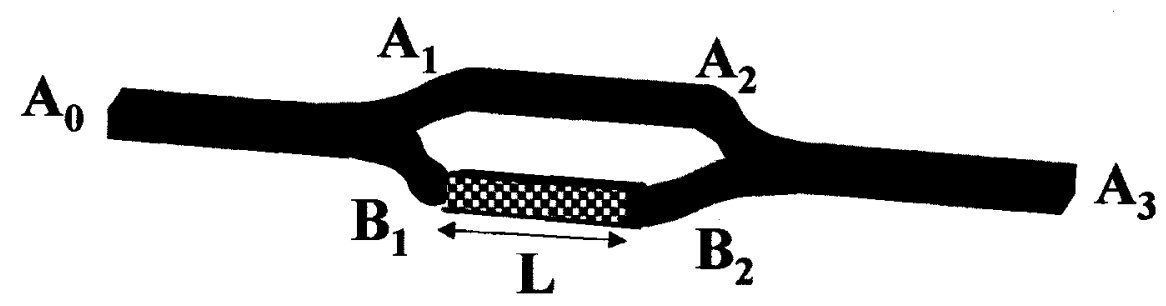

Figure 6.2: Schematic diagram of an MZI-based polarization filter with two $\mathrm{Y}$-junctions as the input and output combiners.

Fig. 6.2 shows a schematic of a simple balanced MZI-based polarization filter with two Y-junctions as the input and output combiners. $A_{i}$ and $B_{j}$ symbolize the electric component of the optical field at particular locations within the MZI device. Randomly polarized incident light is split by the Y-junction and then coupled into the two interferometer arms. The two split beams are identical in magnitude and phase. A patch 
of oxide cladding is then applied to one of the interferometer arms as a phase shifter. Since a balanced MZI is used here, the phase change between the two parallel arms is therefore only induced by the stress. Assuming the oxide patch is applied to the bottom arm, with a length of $L$, the magnitude of the electric fields in the arms right before the recombining Y-junction coupler are

$$
\begin{array}{r}
A_{2}=A_{1} e^{-j \beta l}=\frac{A_{0}}{\sqrt{2}} e^{-j \beta l}, \\
\text { and } B_{2}=B_{1} e^{-j \beta l+j \Delta \phi}=\frac{A_{0}}{\sqrt{2}} e^{-j \beta l+j \Delta \phi},
\end{array}
$$

where the stress induced phase shift is $\Delta \phi=-\frac{2 \pi}{\lambda} \delta n L$ (defined in Eq. 6.2), and $l$ is the total length of each arm. For simplicity, the loss contributions to the overall system performance are not included in the derivations. After recombining the fields $A_{2}$ and $B_{2}$ at the output $\mathrm{Y}$-junction, we obtain a total field

$$
A_{3}=\frac{1}{\sqrt{2}}\left(A_{2}+B_{2}\right)=A_{0} e^{-j\left(\beta l-\frac{\Delta \phi}{2}\right)} \cos \left(\frac{\Delta \phi}{2}\right) .
$$

Thus the optical intensity at the output port becomes:

$$
\left|A_{3}\right|^{2}=\left|A_{0}\right|^{2} \cos ^{2}\left(\frac{\Delta \phi}{2}\right)
$$

In order to achieve a complete polarization filtering with no excess insertion loss, an overall 0 relative phase shift between the 2 arms is required for one polarization, while a $\pi$ shift is desired for the other polarization, as stated in Eq. 6.4. Two scenarios are valid for polarization filtering: (i) TE filter where TM becomes the only output, and (ii) TM filter where TE emerges from the output port. Expressing the conditions mathematically for polarization filtering, we have

$$
\begin{gathered}
\Delta \phi^{\mathrm{TE}}=(2 M+1) \pi ; \quad \Delta \phi^{\mathrm{TM}}=2 N \pi \quad[\text { for TE filters]; } \\
\quad \text { or } \\
\Delta \phi^{\mathrm{TE}}=2 M^{\prime} \pi ; \quad \Delta \phi^{\mathrm{TM}}=\left(2 N^{\prime}+1\right) \pi \quad \text { [for TM filters] }
\end{gathered}
$$


where $M, N, M^{\prime}$, and $N^{\prime}$ are integers. By solving Eqs. 6.9 and 6.8, the oxide patch length requirements for the two polarization filters are:

$$
\begin{aligned}
L=\frac{N \lambda}{\delta n_{\mathrm{TM}}}=\frac{\lambda\left(M+\frac{1}{2}\right)}{\delta n_{\mathrm{TE}}} \quad[\text { for TE filters }] \\
\text { or } \\
L=\frac{M^{\prime} \lambda}{\delta n_{\mathrm{TE}}}=\frac{\lambda\left(N^{\prime}+\frac{1}{2}\right)}{\delta n_{\mathrm{TM}}} \quad[\text { for TM filters]. }
\end{aligned}
$$

The above equations lead to simple relations of the stress-induced index change in TE and TM for effective polarization filtering,

$$
\begin{array}{ll}
\frac{\delta n_{\mathrm{TE}}}{\delta n_{\mathrm{TM}}}=\frac{M+\frac{1}{2}}{N} & \quad[\text { for TE filters }] \\
& \text { or } \\
\frac{\delta n_{\mathrm{TE}}}{\delta n_{\mathrm{TM}}}=\frac{M^{\prime}}{N^{\prime}+\frac{1}{2}} & \quad[\text { for TM filters }]
\end{array}
$$

$M, N, M^{\prime}$, and $N^{\prime}$ must all be integers to achieve a perfect polarization splitting function. For a fixed waveguide cross-sectional geometry, the index changes $\delta n_{\mathrm{TE}, \mathrm{TM}}$ are linearly proportional to the cladding stress, leading to a stress independent ratio $\frac{\delta n_{\mathrm{TE}}}{\delta n_{\mathrm{TM}}}$. It is obvious that the above equations may not have exact solutions. However, an $M$ and $N$ can always be found that approximately satisfy these equations to arbitrary accuracy. Unfortunately, for large $M$ and $N$ the resulting device may be far too long. Thus, the design of the geometry profile needs to be taken into consideration to satisfy the polarization filtering condition defined in Eq. 6.12 or 6.13, for reasonable values of $M$ and $N$. Since the device has a single output port for the device shown in Fig. 6.2, the MZI can only function as a polarization filter.

\subsubsection{MZI with One Y-junction and One Directional Coupler}

To convert a polarization filter into a splitter, a two-port $3 \mathrm{~dB}$ directional coupler is introduced as the output coupler. Fig. 6.3 shows a schematic of the modified MZI polarization splitter with a $\mathrm{Y}$-junction input coupler and a $3 \mathrm{~dB}$ directional coupler as the 
output combiner.

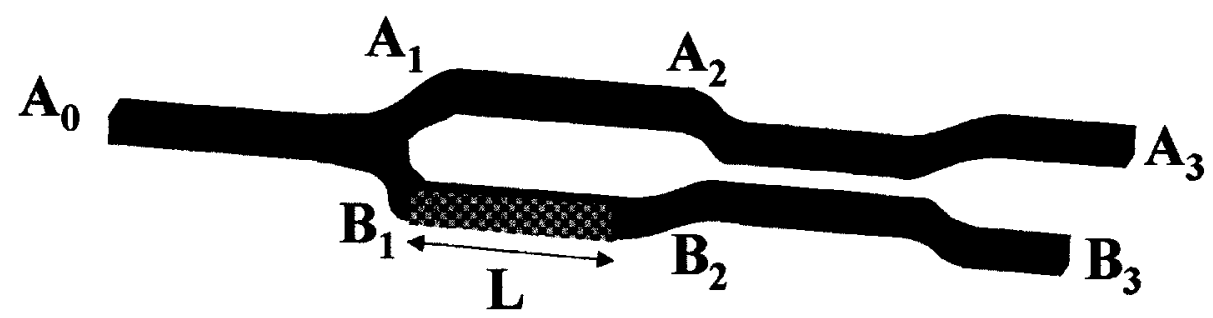

Figure 6.3: Schematic diagram of an MZI-based polarization splitter with one $\mathrm{Y}$-junction input coupler and one $3 \mathrm{~dB}$ directional coupler as the output combiner.

Since the first half of the device is identical to the previous case with a Y-junction input coupler and an oxide patch on the bottom interferometer arm, the relationship defined in Eq. 6.5 for $A_{2}$ and $B_{2}$ can be reused. The two parallel arms then recombine during the output directional coupler. The $3 \mathrm{~dB}$ directional coupler introduces an additional $\pi / 2$ phase shift at the output power transfer. Computing the resulting fields at $A_{3}$ and $B_{3}$, we have

$$
\begin{aligned}
& A_{3}=\cos \left(\kappa l_{c}\right) A_{2}-j \sin \left(\kappa l_{c}\right) B_{2}=\frac{A_{0}}{\sqrt{2}} e^{-j\left(\beta l-\frac{\Delta \phi}{2}\right)}\left[\cos \left(\kappa l_{c}\right) e^{-j \frac{\Delta \phi}{2}}-j \sin \left(\kappa l_{c}\right) e^{j \frac{\Delta \phi}{2}}\right] \\
& B_{3}=\cos \left(\kappa l_{c}\right) B_{2}-j \sin \left(\kappa l_{c}\right) A_{2}=j \frac{A_{0}}{\sqrt{2}} e^{-j\left(\beta l-\frac{\Delta \phi}{2}\right)}\left[\cos \left(\kappa l_{c}\right) e^{j \frac{\Delta \phi}{2}}-j \sin \left(\kappa l_{c}\right) e^{-j \frac{\Delta \phi}{2}}\right]
\end{aligned}
$$

where $\kappa$ and $l_{c}$ are the coupling coefficient and the coupling length of the directional coupler, respectively. For an ideal $3 \mathrm{~dB}$ directional coupler, $\kappa l_{c}=\pi / 4$ is assumed, and the above equations can be simplified to:

$$
\begin{aligned}
& A_{3}=A_{0} e^{-j\left(\beta l-\frac{\Delta \phi}{2}+\frac{\pi}{4}\right)} \cos \left(\frac{\Delta \phi}{2}-\frac{\pi}{4}\right) \\
& B_{3}=j A_{0} e^{-j\left(\beta l-\frac{\Delta \phi}{2}-\frac{\pi}{4}\right)} \sin \left(\frac{\Delta \phi}{2}-\frac{\pi}{4}\right) .
\end{aligned}
$$

Hence, the output power at each output port is given by:

$$
\begin{array}{r}
\left|A_{3}\right|^{2}=\left|A_{0}\right|^{2} \cos ^{2}\left(\frac{\Delta \phi}{2}-\frac{\pi}{4}\right) \quad \text { [Top port] } \\
\left|B_{3}\right|^{2}=\left|A_{0}\right|^{2} \sin ^{2}\left(\frac{\Delta \phi}{2}-\frac{\pi}{4}\right) \quad[\text { Bottom port] }
\end{array}
$$


There are two possible splitting scenarios: (i) TE (or TM) polarized light appears at the top (or bottom) output branch, and (ii) TM (or TE) polarized light emerges from the top (or bottom) output branch. In either case, the two polarization dependent phase differences need to be $\pi$ out of phase. In other words,

Polarization Splitter I: TE (top port) and TM (bottom port):

$$
\Delta \phi^{\mathrm{TE}}-\frac{\pi}{2}=2 M \pi, \Delta \phi^{\mathrm{TM}}-\frac{\pi}{2}=(2 N+1) \pi
$$

or

Polarization Splitter II: TM (top port) and TE (bottom port):

$$
\Delta \phi^{\mathrm{TM}}-\frac{\pi}{2}=2 N^{\prime} \pi, \Delta \phi^{\mathrm{TE}}-\frac{\pi}{2}=\left(2 M^{\prime}+1\right) \pi,
$$

where $M, N, M^{\prime}$, and $N^{\prime}$ are integers.

By solving Eqs. 6.17 and 6.18, the oxide patch length requirements for the two polarization splitting scenarios are:

$$
\begin{array}{r}
L=\frac{\lambda}{\delta n_{\mathrm{TE}}}\left(M+\frac{1}{4}\right)=\frac{\lambda}{\delta n_{\mathrm{TM}}}\left(N+\frac{3}{4}\right) \quad[\mathrm{I}: \mathrm{TE}-\text { top; TM - bottom]; } \\
\text { or } \\
L=\frac{\lambda}{\delta n_{\mathrm{TM}}}\left(N^{\prime}+\frac{1}{4}\right)=\frac{\lambda}{\delta n_{\mathrm{TE}}}\left(M^{\prime}+\frac{3}{4}\right) \quad[\mathrm{II}: \mathrm{TM}-\text { top; TE - bottom]. }
\end{array}
$$

The ratios of the TE and TM index change, $\delta n_{\mathrm{TE}}$ and $\delta n_{\mathrm{TM}}$, must satisfy the following condition for polarization splitting:

$$
\begin{array}{cc}
\frac{\delta n_{\mathrm{TE}}}{\delta n_{\mathrm{TM}}}=\frac{M+\frac{1}{4}}{N+\frac{3}{4}}, & {[\mathrm{I}: \mathrm{TE}-\text { top; TM - bottom }] ;} \\
\text { or } \\
\frac{\delta n_{\mathrm{TE}}}{\delta n_{\mathrm{TM}}}=\frac{M^{\prime}+\frac{3}{4}}{N^{\prime}+\frac{1}{4}} & {[\text { III: TM - top; TE - bottom }] .}
\end{array}
$$

Again, $M, N, M^{\prime}$, and $N^{\prime}$ must be all integers for a perfect polarization splitting function. Due to the additional $\pi / 2$ phase change introduced by the output directional coupler, the index ratios and oxide patch length requirements differ from the conditions defined in Eqs. 6.12-6.11 for the MZI with two Y-junctions. 


\subsubsection{MZI with One Y-junction and One MMI Coupler}

The previous subsection described a polarization splitter with a directional coupler as the output combiner. Directional couplers are known for their poor tolerances in fabrication. In addition, directional couplers have polarization dependent performance because the coupler coefficients ( $\kappa$ in Eq. 6.14) of the TE and TM polarization are different. In this subsection, a splitter design involving a two-port $3 \mathrm{~dB}$ multimode interference (MMI) coupler is proposed (as shown in Fig. 6.4). The main advantages of an MMI coupler over a directional coupler is the ease in fabrication and in insensitivity to polarization [70].

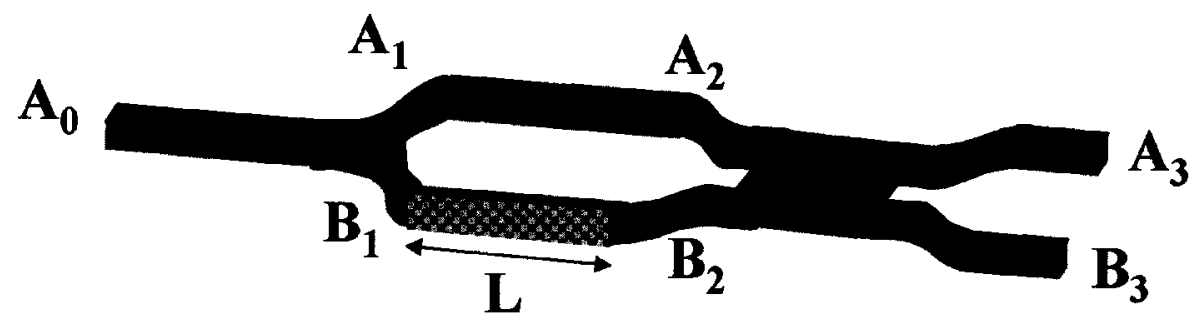

Figure 6.4: Schematic diagram of an MZI-based polarization splitter with a Y-junction input coupler and an MMI output coupler.

Similar to the previous two cases, the fields at $\mathrm{A}_{2}$ and $\mathrm{B}_{2}$ are given by Eq. 6.5 with the inclusion of the stress-induced phase change. A typical MMI coupler is used here as a $3 \mathrm{~dB}$ output coupler, as illustrated in Fig. 6.5(a). The MMI coupler has a slab width $W_{\mathrm{MMI}}$, length $L_{\mathrm{MMI}}$, and the input/output ports are symmetrically placed with a separation $S_{\mathrm{MMI}}=W_{\mathrm{MMI}} / 3$ apart from each other. The principle of the MMI devices is based on the self-imaging property of multimode waveguides [93]. An input field profile is reproduced in single or multiple images at periodic intervals along the propagation direction of the guide due to the destructive/constructive interferences among the large number of modes in the MMI slab. Light intensity patterns corresponding to a $3 \mathrm{~dB}$ MMI coupler are shown in Fig. 6.5(b). The design details of a $3 \mathrm{~dB}$ MMI coupler can be found in Refs. [70,93].

Similar to a $3 \mathrm{~dB}$ directional coupler, the MMI coupler produces two output fields 


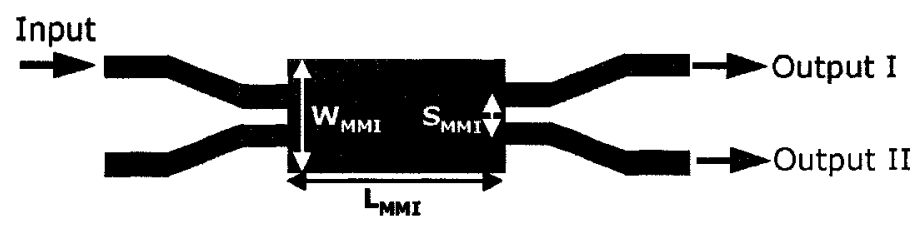

(a)

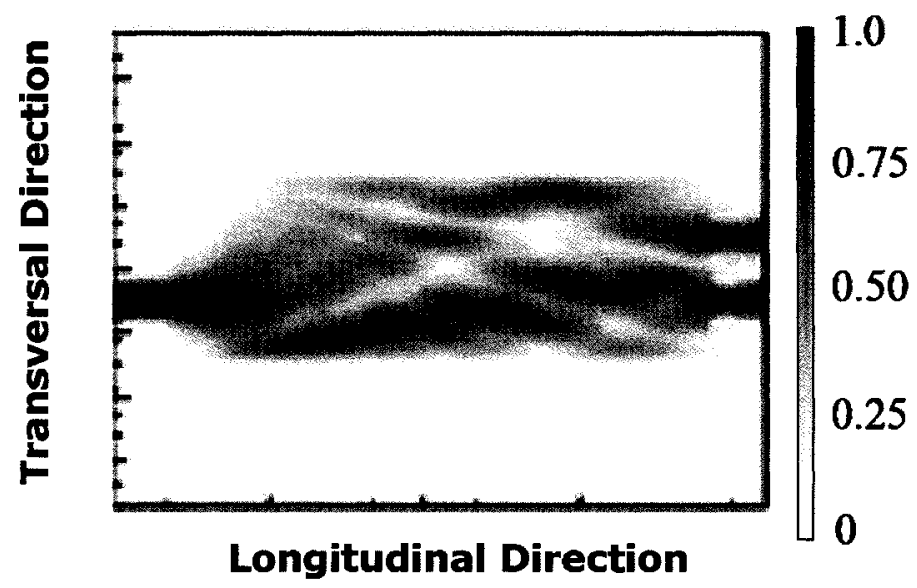

(b)

Figure 6.5: A $2 \times 2 \mathrm{MMI}$ coupler: (a) the schematic layout; (b) the light intensity patterns along the longitudinal and transversal directions, producing two-fold images [70].

with the same magnitude but $\pi / 2$ out-of-phase. The output field $A_{3}$ and $B_{3}$ are:

$$
\begin{gathered}
A_{3}=\frac{1}{\sqrt{2}}\left(A_{2} e^{\phi_{\mathrm{MMI}}+\frac{3 \pi}{8}}+B_{2} e^{\phi_{\mathrm{MMI}}-\frac{\pi}{8}}\right)=A_{0} e^{-j\left(\beta l-\frac{\Delta \phi}{2}-\phi_{\mathrm{MMI}}-\frac{\pi}{8}\right)} \cos \left(\frac{\Delta \phi}{2}-\frac{\pi}{4}\right) ; \\
B_{3}=\frac{1}{\sqrt{2}}\left(A_{2} e^{\phi_{\mathrm{MMI}}-\frac{\pi}{8}}+B_{2} e^{\phi_{\mathrm{MMI}}+\frac{3 \pi}{8}}\right)=j A_{0} e^{-j\left(\beta l-\frac{\Delta \phi}{2}-\phi_{\mathrm{MMI}}-\frac{5 \pi}{8}\right)} \sin \left(\frac{\Delta \phi}{2}-\frac{\pi}{4}\right),
\end{gathered}
$$

where $\phi_{\mathrm{MMI}}$ is a constant phase associated with the MMI and is a function of the MMI length $L_{\mathrm{MMI}}[93]$ :

$$
\begin{aligned}
\phi_{\mathrm{MMI}} & =-\frac{2 \pi n}{\lambda} L_{\mathrm{MMI}}+\frac{\pi}{24}, \\
L_{\mathrm{MMI}} & \approx \frac{2 n W_{e}^{2}}{3 \lambda_{0}} .
\end{aligned}
$$

The output power at each output port is:

$$
\begin{array}{r}
\left|A_{3}\right|^{2}=\left|A_{0}\right|^{2} \cos ^{2}\left(\frac{\Delta \phi}{2}-\frac{\pi}{4}\right) \quad \text { [Top port] } \\
\left|B_{3}\right|^{2}=\left|A_{0}\right|^{2} \sin ^{2}\left(\frac{\Delta \phi}{2}-\frac{\pi}{4}\right) \quad \text { [Bottom port]. }
\end{array}
$$


The phase requirements for obtaining the maximum output powers are the same as those given in Eqs. 6.17-6.18. Therefore the relations defined in Eqs. 6.21-6.20 also apply in this case for achieving a full polarization splitting function.

\subsubsection{MZI with Two Symmetric Directional Couplers}

The combination of one Y-junction input coupler with either a directional coupler or an MMI coupler produces a polarization splitter. Here we introduce a symmetric MZI structure with two identical directional couplers. Fig. 6.6 shows a schematic of a balanced MZI with two $3 \mathrm{~dB}$ directional couplers.

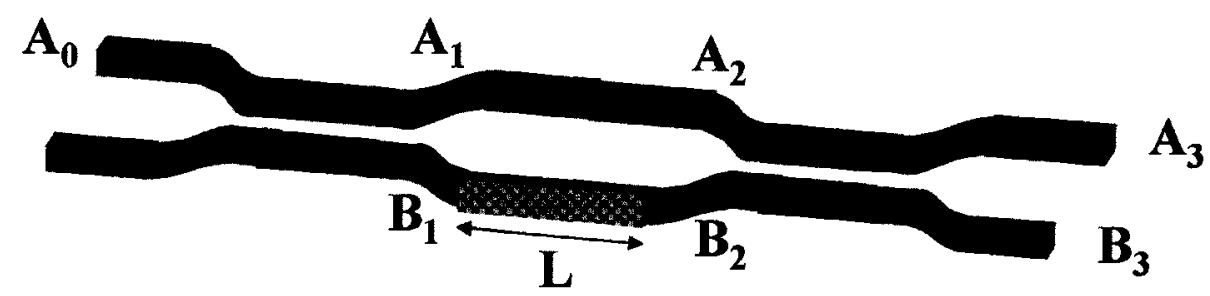

Figure 6.6: Schematic diagram of an MZI-based polarization splitter with two $3 \mathrm{~dB}$ directional couplers as both the input and the output combiners.

Both $3 \mathrm{~dB}$ directional couplers are assumed to be ideal, with a coupling length $l_{c}$ that satisfies $\kappa l_{c}=\pi / 4$. After the input passes the first $3 \mathrm{~dB}$ directional coupler, the total electric fields right before the second coupler are:

$$
\begin{array}{r}
A_{2}=A_{1} e^{-j \beta l}=\frac{A_{0}}{\sqrt{2}} e^{-j \beta l}, \\
B_{2}=B_{1} e^{-j \beta l+j \Delta \phi}=-j \frac{A_{0}}{\sqrt{2}} e^{-j \beta l+j \Delta \phi}
\end{array}
$$

where $\Delta \phi=-\frac{2 \pi}{\lambda} \delta n L$ and $l$ is the total length of the arms. With the addition of a $\pi / 2$ phase shift from the second directional coupler, the output fields become:

$$
\begin{aligned}
& A_{3}=\frac{1}{\sqrt{2}} A_{2}-j \frac{1}{\sqrt{2}} B_{2}=-j A_{0} e^{-\left(j \beta l+\frac{\Delta \phi}{2}\right)} \sin \frac{\Delta \phi}{2} \\
& B_{3}=\frac{1}{\sqrt{2}} B_{2}-j \frac{1}{\sqrt{2}} A_{2}=-j A_{0} e^{-j\left(\beta l+\frac{\Delta \phi}{2}\right)} \cos \frac{\Delta \phi}{2} .
\end{aligned}
$$


The resulting powers at the two output ports are:

$$
\begin{array}{r}
\left|A_{3}\right|^{2}=\left|A_{0}\right|^{2} \sin ^{2}\left(\frac{\Delta \phi}{2}\right) \quad[\text { Top port }] \\
\left|B_{3}\right|^{2}=\left|A_{0}\right|^{2} \cos ^{2}\left(\frac{\Delta \phi}{2}\right) \quad[\text { Bottom port }] ;
\end{array}
$$

It is confirmed from the above equations that a maximum power of $\left|A_{0}\right|^{2}$ for each of the two polarizations is possible. It is noted that the derivation assumes no loss in the directional couplers.

Again, there are two scenarios for polarization splitting:

Polarization Splitter I: TE (top port) and TM (bottom port):

$$
\begin{gathered}
\Delta \phi^{\mathrm{TE}}=(2 M+1) \pi, \quad \Delta \phi^{\mathrm{TM}}=2 N \pi \\
\text { or }
\end{gathered}
$$

Polarization Splitter II: TM (top port) and TE (bottom port):

$$
\Delta \phi^{\mathrm{TE}}=2 M^{\prime} \pi, \quad \Delta \phi^{\mathrm{TM}}=\left(2 N^{\prime}+1\right) \pi,
$$

where $M, N, M^{\prime}$, and $N^{\prime}$ are integers.

The oxide patch length necessary for polarization splitting is:

$$
\begin{array}{ll}
L=\frac{N \lambda}{\delta n_{\mathrm{TM}}}=\frac{\lambda\left(M+\frac{1}{2}\right)}{\delta n_{\mathrm{TE}}} & {[\mathrm{I}: \mathrm{TE}-\text { top; TM - bottom }]} \\
& \text { or } \\
L=\frac{M^{\prime} \lambda}{\delta n_{\mathrm{TE}}}=\frac{\lambda\left(N^{\prime}+\frac{1}{2}\right)}{\delta n_{\mathrm{TM}}} & {[\mathrm{II}: \mathrm{TM}-\text { top; TE - bottom }] .}
\end{array}
$$

To achieve a full polarization splitting function, the ratio $\frac{\delta n_{\mathrm{TE}}}{\delta n_{\mathrm{TM}}}$ must satisfy:

$$
\begin{array}{rc}
\frac{\delta n_{\mathrm{TE}}}{\delta n_{\mathrm{TM}}}=\frac{M+\frac{1}{2}}{N}, & {[\mathrm{I}: \mathrm{TE}-\text { top; TM - bottom }] ;} \\
\text { or } \\
\frac{\delta n_{\mathrm{TE}}}{\delta n_{\mathrm{TM}}}=\frac{M^{\prime}}{N^{\prime}+\frac{1}{2}} & {[\text { III: TM - top; TE - bottom }] .}
\end{array}
$$

The conditions for the MZI with symmetric directional couplers are similar to those defined in Eqs. 6.12-6.11 for the case of the MZI with symmetric Y-junctions. 


\subsubsection{MZI with Two Symmetric MMI Couplers}

Finally, with a replacement of the $3 \mathrm{~dB}$ couplers with two identical MMI couplers, a symmetric polarization splitter design based on a balanced MZI with two 3dB MMI input/output couplers is illustrated in Fig. 6.7. Here the $2 \times 2$ restricted MMI coupler

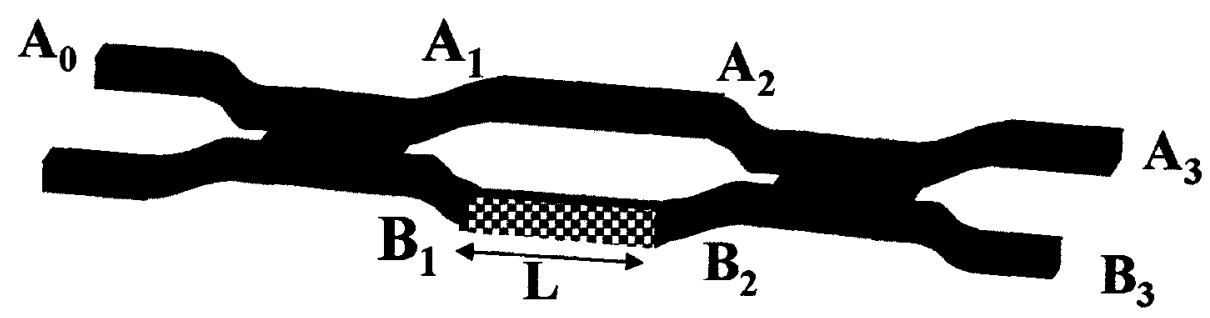

Figure 6.7: Schematic diagram of an MZI-based polarization splitter with MMI couplers as both the input and the output combiners.

(similar to the one described in Figs. 6.4 and 6.5(a)) is used. When an incoming field propagates through the upper input port, we expect the fields at output ports of the first MMI to be:

$$
\begin{gathered}
A_{1}=\frac{A_{0}}{\sqrt{2}} e^{j\left(\phi_{\mathrm{MMI}}+\frac{3 \pi}{8}\right)}, \\
B_{1}=\frac{A_{0}}{\sqrt{2}} e^{j\left(\phi_{\mathrm{MMI}}-\frac{\pi}{8}\right)},
\end{gathered}
$$

where $\phi_{\text {MMI }}$ was defined in Eq. 6.24. This $3 \mathrm{~dB}$ MMI coupler will produce two output fields with the same magnitude, but with a $\pi / 2$ phase difference, similar to the $3 \mathrm{~dB}$ directional coupler discussed earlier.

If a compensating oxide patch is placed in the lower branch of the MZI, the electric fields at $A_{2}$ and $B_{2}$ can be expressed as,

$$
\begin{array}{r}
A_{2}=\frac{A_{0}}{\sqrt{2}} e^{-j\left(\beta l-\phi_{\mathrm{MMI}}-\frac{3 \pi}{8}\right)} \\
B_{2}=\frac{A_{0}}{\sqrt{2}} e^{-j\left(\beta l-\phi_{\mathrm{MMI}}+\frac{\pi}{8}-\Delta \phi\right)} .
\end{array}
$$

When the fields $A_{2}$ and $B_{2}$ go through the second MMI coupler, an additional $\pi / 2$ phase 
shift is introduced by the coupler, and the fields at the two output ports become:

$$
\begin{aligned}
& A_{3}=\frac{A_{2}}{\sqrt{2}} e^{j\left(\phi_{\mathrm{MMI}}+\frac{3 \pi}{8}\right)}+\frac{B_{2}}{\sqrt{2}} e^{j\left(\phi_{\mathrm{MMI}}-\frac{\pi}{8}\right)}=A_{0} e^{-j\left(\beta l-2 \phi_{\mathrm{MMI}}+\frac{\pi}{4}+\frac{\Delta \phi}{2}\right)} \sin \frac{\Delta \phi}{2} \\
& B_{3}=\frac{A_{2}}{\sqrt{2}} e^{j\left(\phi_{\mathrm{MMI}}-\frac{\pi}{8}\right)}+\frac{B_{2}}{\sqrt{2}} e^{j\left(\phi_{\mathrm{MMI}}+\frac{3 \pi}{8}\right)}=A_{0} e^{-j\left(\beta l-2 \phi_{\mathrm{MMI}}-\frac{\pi}{4}+\frac{\Delta \phi}{2}\right)} \cos \frac{\Delta \phi}{2}
\end{aligned}
$$

Thus, the output powers at the two ports defined in Eq. 6.28 for two directional couplers are still valid for this case. The phase and index change relations described in Eqs. 6.29-6.34 are necessary for the polarization splitting in an MZI with two identical MMI couplers.

To summarize, the different configurations of the MZI-based polarization splitters can be categorized into two groups: (i) a symmetric type (e.g., an MZI with two identical $3 \mathrm{~dB}$ MMIs or with two directional couplers); (ii) an asymmetric type (e.g., with one Y-junction and one directional coupler or with one Y-junction and one MMI). The associated oxide patch lengths and the corresponding polarization dependent index ratio conditions which are required for achieving polarization splitting can be expressed as:

Symmetric type (with two MMIs or two directional couplers):

Splitter I: TE (top output); TM (bottom output)

$$
\frac{\delta n_{\mathrm{TE}}}{\delta n_{\mathrm{TM}}}=\frac{M+\frac{1}{2}}{N} \text { and } L=\frac{N \lambda}{\delta n_{\mathrm{TM}}}=\frac{\lambda\left(M+\frac{1}{2}\right)}{\delta n_{\mathrm{TE}}}
$$

or

Splitter II: TM (top output); TE (bottom output)

$$
\frac{\delta n_{\mathrm{TE}}}{\delta n_{\mathrm{TM}}}=\frac{M^{\prime}}{N^{\prime}+\frac{1}{2}} \text { and } L=\frac{M^{\prime} \lambda}{\delta n_{\mathrm{TE}}}=\frac{\lambda\left(N^{\prime}+\frac{1}{2}\right)}{\delta n_{\mathrm{TM}}} .
$$

Asymmetric type (with 1 Y-junction + 1 directional coupler or 1 Y-junction 
$+1 M M I):$

Splitter I: TE (top output); TM (bottom output)

$$
\frac{\delta n_{\mathrm{TE}}}{\delta n_{\mathrm{TM}}}=\frac{M+\frac{1}{4}}{N+\frac{3}{4}} \text { and } L=\frac{\lambda\left(M+\frac{1}{4}\right)}{\delta n_{\mathrm{TE}}}=\frac{\lambda\left(N+\frac{3}{4}\right)}{\delta n_{\mathrm{TM}}}
$$

or

Splitter II: TM (top output); TE (bottom output)

$$
\frac{\delta n_{\mathrm{TE}}}{\delta n_{\mathrm{TM}}}=\frac{M^{\prime}+\frac{3}{4}}{N^{\prime}+\frac{1}{4}} \text { and } L=\frac{\lambda\left(N^{\prime}+\frac{1}{4}\right)}{\delta n_{\mathrm{TM}}}=\frac{\lambda\left(M^{\prime}+\frac{3}{4}\right)}{\delta n_{\mathrm{TE}}} .
$$

\subsection{Design Considerations}

\subsubsection{Waveguide Cross-section}

Silicon-on-insulator (SOI) wafers have been chosen as the platform of our waveguide devices. The wafers used in this project have a silicon core thickness of $H=2.2 \mu \mathrm{m}$ and a buried oxide bottom cladding of $0.4 \mu \mathrm{m}$, which are the same as the waveguides that were used for the fabricated AWG test devices discussed in Chapter 5.

It is noted that the ridge waveguides considered in our analyses usually have deep etch depths. There are two reasons behind this choice. One is that a deeply etched ridge offers a tighter light confinement in the waveguide and further reduces the device footprint. The second reason is that the stress distribution present in the vicinity of the ridge provides a larger effect on waveguide polarization in a deeply etched ridge waveguide. As observed in Fig. 4.15, shallow etched designs have smaller stress-induced effects on mode characteristics and polarization tuning. The target etch depths in our experiments are 1.2 and $1.4 \mu \mathrm{m}$. It is evident from Fig. 4.15 that the relative contribution of the stress-induced effects on total birefringence at $D=1.4 \mu \mathrm{m}$ can be $50 \%$ stronger than that at $D=1.2 \mu \mathrm{m}$, for a ridge core with a $2.2 \mu \mathrm{m}$ thickness.

As discussed in the previous chapter and illustrated in Figs. 4.8 and 4.17, the waveguide aspect ratio $W / H$ plays an important role in determining the polarization sensitivity 
to ridge dimensions. With a fixed core thickness $H$, wider waveguides are less susceptible to dimension fluctuations (e.g., Fig. 4.16(b)), which requires less precision in fabrication control. On average, our in-house fabrication precision in waveguide width is $\sim \pm 0.1 \mu \mathrm{m}$. Two widths have been chosen: $1.5 \mu \mathrm{m}$ and $2.0 \mu \mathrm{m}$.

Single-mode ridge waveguides are required for devices based on interference such as the MZI-based polarization splitters. However, single-mode waveguides require shallower etch depths, which reduce the effects of the cladding induced stress on the guiding core. We use Soref's single-mode condition defined in Eq. 1.6 as a basic guideline for the choices of the ridge etch depth. The final etch depth design of $1.2 \mu \mathrm{m}$ offers the best compromise between optimizing the cladding induced stress and achieving the singlemode operation. We have also included designs with deeper etch depths of $1.4 \mu \mathrm{m}$ for devices with Y-junctions and MMI $3 \mathrm{~dB}$ couplers.

With the given choices of the ridge width and the etch depth, both the effective indices of the TE and TM polarized mode and the overall waveguide birefringence can be changed significantly by depositing a stressed cladding film. Experimentally, stress in a standard PECVD deposited $\mathrm{SiO}_{2}$ film ranges from $\sigma_{\text {film }}=-100$ to $-400 \mathrm{MPa}$. For simulation purposes, we choose $\sim-330 \mathrm{MPa}$ as the target stress level which is well below the maximum stress obtainable from the actual fabrication. It is shown in Fig. 4.12 that $\Delta n_{\text {stress }}$ saturates and approaches a maximum level as the oxide thickness increases beyond the ridge etch depth. The deposition thickness of the oxide cladding is deliberately chosen to be away from the "saturation" region. The nominal design of the cladding thickness is $t=1 \mu \mathrm{m}$. The oxide thickness covering the ridge sidewalls is assumed to be $70 \%$ of that on the top of the waveguiding layer, as measured in our upper oxide cladding film [89,92]. As predicted in Fig. 4.11 and 4.12, a compressive stress $\sigma_{\text {film }}$ of $-330 \mathrm{MPa}$ can induce a change in both the effective index $\left(\delta n_{\mathrm{eff}}\right)$ and birefringence $\left(\Delta n_{\text {stress }}\right)$ on the order of $10^{-3}$. We recall that the propagation phase of an optical mode is $\phi=2 \pi n_{\text {eff }} L / \lambda$, where $L$ is the propagation length. A change of $10^{-3}$ in $\delta n_{\text {eff }}$ 
yields a complete $\pi$ phase change after a propagation distance of $L=775 \mu \mathrm{m}$ (assuming $\lambda=1550 \mathrm{~nm}$ ). Since the TE and TM modes of the ridge waveguides considered in this study have very different stress-induced effective indices (i.e., $\delta n_{\text {eff }}^{\mathrm{TE}} \neq \delta n_{\text {eff }}^{\mathrm{TM}}$ ), the manipulation of the phase changes between the two orthogonal modes allows effective polarization splitting.

Fig. 6.8 summarizes the waveguide design parameters of the ridge cross-section geometry used in our polarization splitter devices. The final waveguide cross-section dimensions offer a compromise among the geometry and stress-induced effects and the existing fabrication challenges.

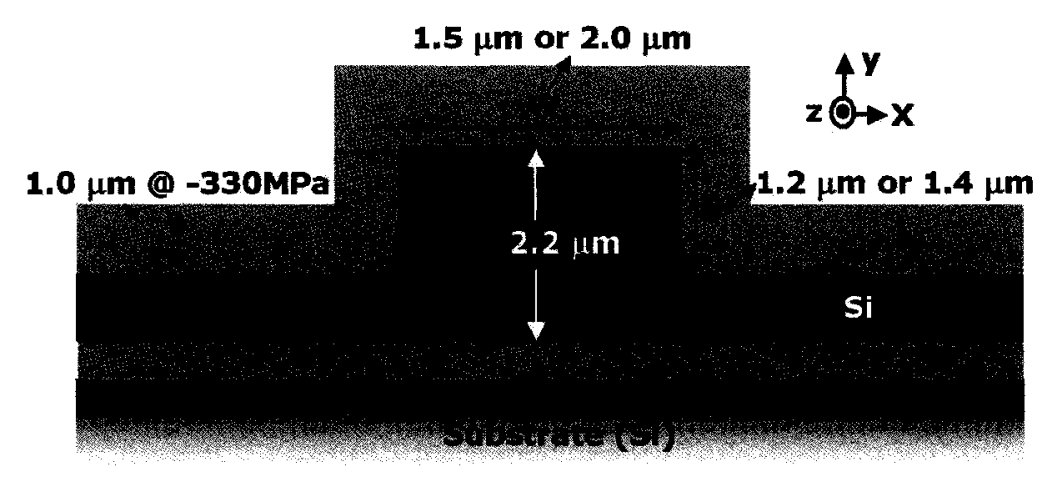

Figure 6.8: Summary of ridge cross-section dimensions for the ridge waveguides used in our polarization splitter devices.

\subsection{2 $3 \mathrm{~dB}$ Couplers}

One of the key elements in an MZI configuration is the $3 \mathrm{~dB}$ coupler. Three basic types of couplers have been examined: directional couplers, $\mathrm{Y}$-junctions, and MMIs. Directional couplers are known for their poor fabrication tolerances and polarization dependent coupling. To achieve a 50/50 split between the two polarizations, the ridge waveguides used in a directional coupler must be single mode. Fabrication tolerances are extremely stringent: the separation between the waveguides in the coupling region and the total coupling length are critical to the coupler performance of a directional coupler. 
The $Y$-junction is simple in concept, however, care must be taken to ensure the split angle is appropriate for low-loss propagation. It is common practice to restrict the split angle to below $2^{\circ}$. In addition, there is a fabrication challenge in making a lossless Y-split. To ensure reproducible fabrication and consistent splitting, a small patch is applied to cover the split region, as illustrated in Fig. 6.9. Out of all three types of $3 \mathrm{~dB}$ couplers, MMIs

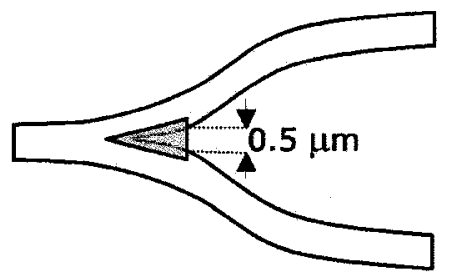

Figure 6.9: Triangular patch to cover the split region for $\mathrm{Y}$-junction fabrication.

are the preferred choice because of their compact size, low insertion loss, large optical bandwidth, ease of fabrication and polarization insensitivity [70,93]. The polarization and wavelength insensitivity of an MMI device are simulated in Fig. 6.10. With the given $3 \mathrm{~dB}$ MMI structure, both polarizations are evenly split into the two output ports with a maximum of $0.6 \%$ difference. Moreover, the splitting is independent of the operating wavelength ranging from $1460 \mathrm{~nm}$ to $1580 \mathrm{~nm}$ (with a maximum variation below $0.7 \%$ ).

It was mentioned earlier that the single-mode waveguides are required for devices based on interference. The etch depth of $D=1.2 \mu \mathrm{m}$ offers the best compromise between optimizing the stress-induced effects and satisfying the single-mode condition. However, with this etch depth, a $3 \mathrm{~dB}$ directional coupler requires a coupling length of over $3.3 \mathrm{~mm}$ with a coupling spacing of $\sim 1.5 \mu \mathrm{m}$. Precise control in the fabrication of the coupling length and spacing are necessary for the full splitting of TE and TM polarizations. We have included designs with deeper etch depths of $1.4 \mu \mathrm{m}$ for devices with Y-junctions and MMI $3 \mathrm{~dB}$ couplers. However, these deeply etched waveguides may exhibit multimode characteristics in the coupling region which may affect the splitting or filtering perfor- 


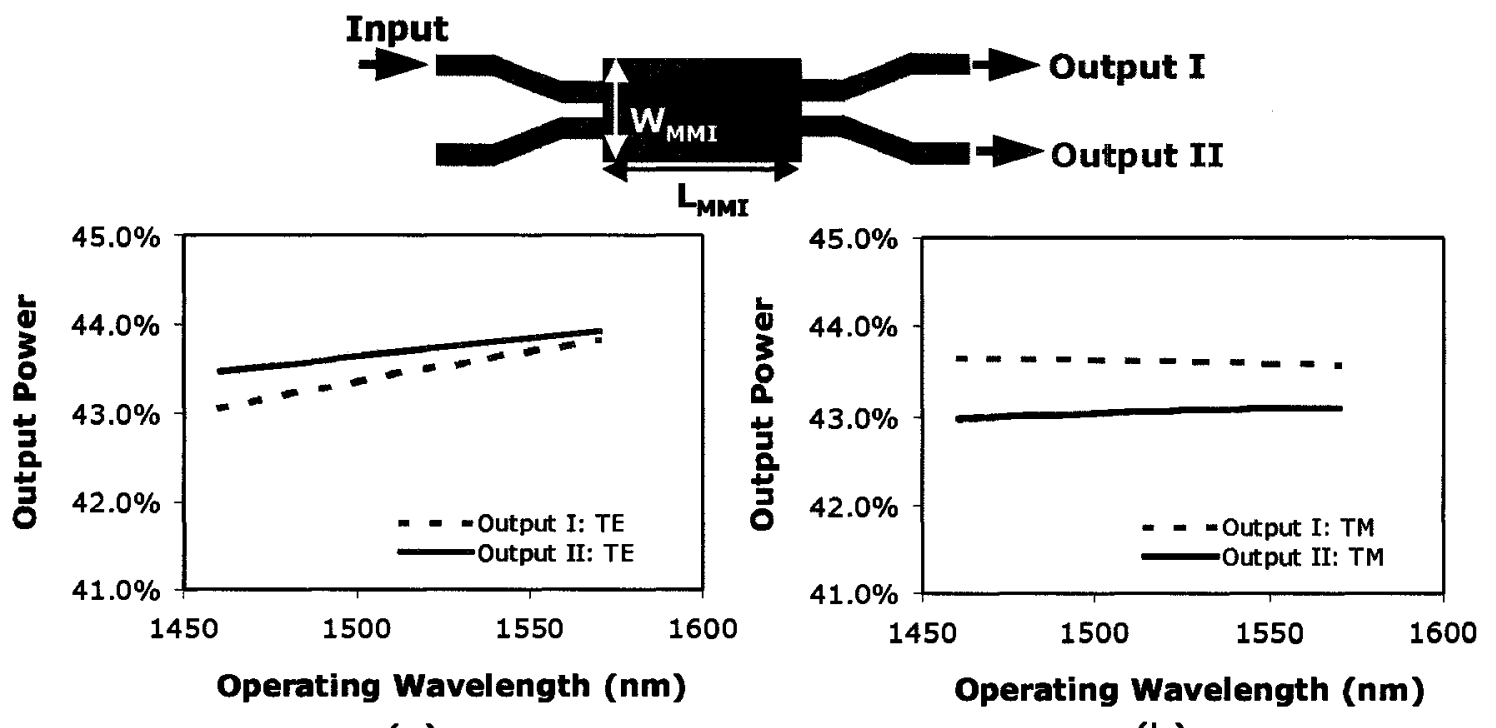

(a)

(b)

Figure 6.10: Polarization and wavelength insensitive performance of a 3 $\mathrm{dB}$ MMI device. The example MMI has the following dimensions: $L_{\mathrm{MMI}}=$ $186 \mu \mathrm{m}$ and $W_{\mathrm{MMI}}=10.5 \mu \mathrm{m}$.

mance. Table 6.1 lists the target parameters of the input and output $3 \mathrm{~dB}$ couplers in various configurations.

\begin{tabular}{|c|c|c|c|c|c|}
\hline \multicolumn{6}{|c|}{3 dB Coupler } \\
\hline \multirow{3}{*}{ MMI } & Width ( $W_{\text {MMI }}$ ) & Length ( $L_{\text {mmI }}$ ) & Spacing ( S $\left._{\text {MMI }}\right)$ & Etch Depth (D) & Fanout Bend Radius (R) \\
\hline & $10.5 \mu \mathrm{m}$ & $167 \mu \mathrm{m}$ & $3.5 \mu \mathrm{m}$ & $1.4 \mu \mathrm{m}$ & $1000 \mu \mathrm{m}$ \\
\hline & $15.3 \mu \mathrm{m}$ & $361 \mu \mathrm{m}$ & $5.1 \mu \mathrm{m}$ & $1.2 \mu \mathrm{m}$ & $1000 \mu \mathrm{m}$ \\
\hline \multirow{2}{*}{ Directional Coupler } & Ridge Width (W) & \multicolumn{2}{|c|}{ Coupling Length/spacing $\left(\mathrm{L}_{\mathrm{c}} / \mathrm{S}_{\mathrm{DC}}\right)$} & Etch Depth (D) & Fanout Bend Radius (R) \\
\hline & $1.5 \mu \mathrm{m}$ & $3303 \mu \mathrm{m}$ & $3.5 \mu \mathrm{m}$ & $1.2 \mu \mathrm{m}$ & $1000 \mu \mathrm{m}$ \\
\hline \multirow{2}{*}{ Y-junction } & \multicolumn{4}{|c|}{ Split Angle } & Fanout Bend Radius ( $R$ ) \\
\hline & \multicolumn{4}{|c|}{$\max .2^{\circ}$} & $1000 \mu \mathrm{m}$ \\
\hline
\end{tabular}

Table 6.1: Design parameters for the $3 \mathrm{~dB}$ input and output couplers used in MZI-based polarization splitters and filters.

\subsection{Simulation Results}

The previous section set the basic design parameters for the ridge waveguides which are used in our MZI-based polarization splitters/filters. The performance of these devices is simulated and presented in this section.

Chapter 6.2 described the different configurations of MZI-based polarization splitters, 
and listed the conditions required for polarization splitting or filtering. The specific stressinduced index ratio $\left(\delta n_{\mathrm{TE}} / \delta n_{\mathrm{TM}}\right)$ must satisfy Eq. 6.33 or 6.34 , while the corresponding oxide patch length $(L)$ needs to fulfill Eq. 6.31 or 6.32 , for the symmetric MZI-based devices (with two identical) couplers. Similarly, the devices with asymmetric couplers (e.g., a Y-junction and an MMI or a directional coupler) are required to meet the relations stated in Eqs. 6.19- 6.22.

For the given ridge waveguide dimensions (height $H=2.2 \mu \mathrm{m}$, width $W=1.5 \mu \mathrm{m}$, etch depth $D=1.4 \mu \mathrm{m}$ ), the choice of the oxide cladding thickness $(t)$ fixes the stressinduced index ratio $\left(\delta n_{\mathrm{TE}} / \delta n_{\mathrm{TM}}\right)$. The cladding stress level $\left(\sigma_{\mathrm{flm}}\right)$ is then used to determine the oxide patch length $L$ for polarization splitting. Figs. 6.11(a) and (b) shows the simulated TE and TM power splitting as a function of the upper oxide cladding patch length $L$ at the operating wavelength of $1550 \mathrm{~nm}$, assuming thickness $t=1 \mu \mathrm{m}$ and $\sigma_{\text {film }}=-330 \mathrm{MPa}$ to match the measured stress in our PECVD oxide films. A reasonable oxide patch length of $686 \mu \mathrm{m}$ will achieve a polarization splitting with TM polarized light appearing at the top port and TE polarized light in the bottom port. For the same device, an oxide patch of $L=938 \mu \mathrm{m}$ is necessary to split TE to the top output port and TM to the bottom port.

To illustrate the wavelength dependence of the device for an oxide patch length of $L=686 \mu \mathrm{m}$, in Fig. 6.12 we plot the output power of the TM and the TE modes in the output ports as a function of the operating wavelength. The splitting extinction ratio is better than $-20 \mathrm{~dB}$ for both ports across the entire C-Band, but the maximum extinction for TE and TM polarized light does not occur at the same wavelength. When the index change condition $\frac{\delta n_{\mathrm{TE}}}{\delta n_{\mathrm{TM}}}=\frac{M+\frac{1}{2}}{N}$ (Eq. 6.33) is satisfied perfectly at a given wavelength, all the TE power will appear at the top output port while all TM will be in the bottom, and the extinction minima of the residual TM (or TE) in the top (or bottom) should overlap. However, it is difficult to fully satisfy the condition in Eq. 6.33, with a reasonably short oxide patch length (i.e., small $M$ and $N$ values). Here we only approximately satisfy 


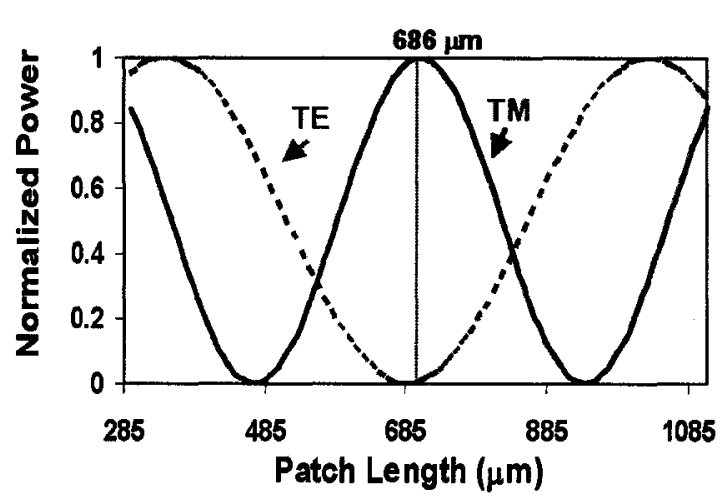

(a)

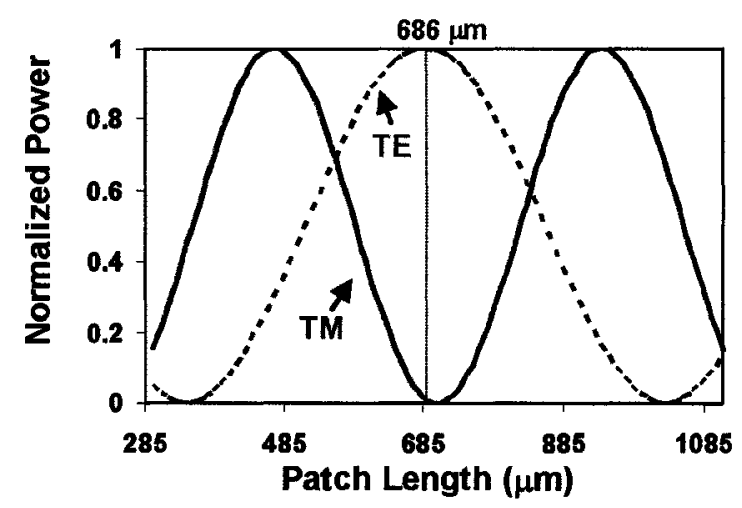

(b)

Figure 6.11: An MZI-based polarization splitter with symmetric $3 \mathrm{~dB}$ couplers: shown are the simulated TE and TM power in the polarization splitter output ports [(a) top port and (b) bottom port] as a function of the upper oxide cladding patch length. The stress is $\sigma_{\text {film }}=-330 \mathrm{MPa}$ and the cladding thickness $t$ is $1 \mu \mathrm{m}$. The ridge waveguides have the following dimensions: $H=2.2 \mu \mathrm{m}, W=1.5 \mu \mathrm{m}$, and $D=1.4 \mu \mathrm{m}$.

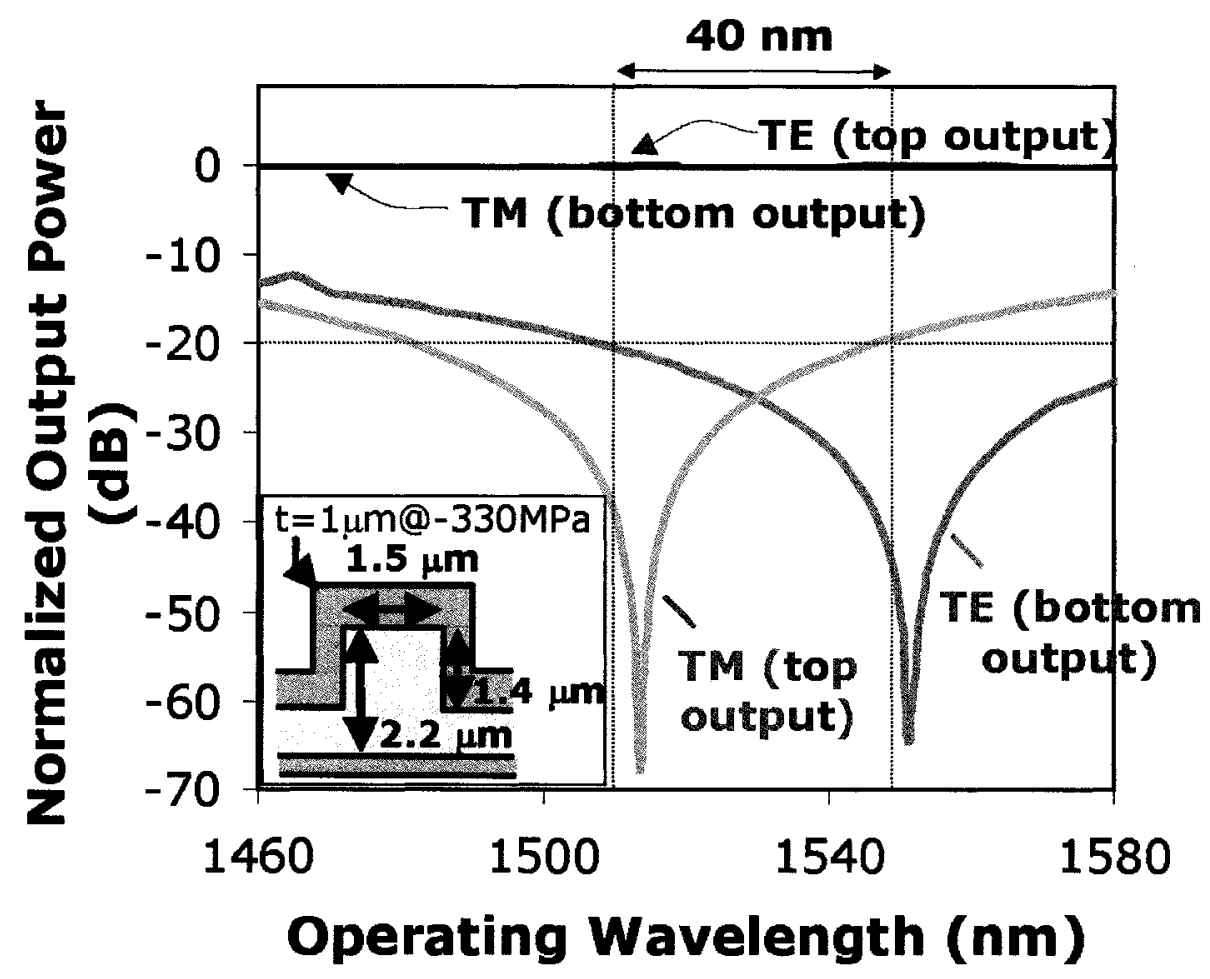

Figure 6.12: Output power of the TE and TM modes as a function of operating wavelength in an MZI-based polarization splitter with symmetric $3 \mathrm{~dB}$ couplers. The same device parameters defined in Fig. 6.11 are used here. The oxide patch length $L$ is set to $686 \mu \mathrm{m}$. 
the condition using a combination of the smallest possible $M$ and $N$ integers that give acceptable performance. Hence, a wavelength shift is observed in the minima of the two residual polarizations, as illustrated in Fig. 6.12. Nevertheless, with this imperfect matching, a $-20 \mathrm{~dB}$ splitting extinction ratio can still be obtained within the $40 \mathrm{~nm}$ bandwidth ranging from $1510 \mathrm{~nm}$ to $1560 \mathrm{~nm}$.

Based on the simulations of the geometrical and stress-induced effects, the nominal parameters of the ridge waveguides and the oxide patches involved in the MZI-based polarization splitters are summarized in Table 6.2. It is noted that there should be two splitting scenarios for each device (i.e., TE at the top output and TM at the bottom output, or vice versa). Here only the designs with the shortest patch length $L$ are shown.

\begin{tabular}{|c|c|c|c|c|c|}
\hline \multirow{2}{*}{ Ridge } & \multirow{2}{*}{$\begin{array}{c}\text { Width (W) } \\
\text { Etch (D) }\end{array}$} & \multicolumn{2}{|c|}{$1.5 \mu \mathrm{m}$} & \multicolumn{2}{|c|}{$2.0 \mu \mathrm{m}$} \\
\hline & & $1.2 \mu \mathrm{m}$ & $1.4 \mu \mathrm{m}$ & $1.2 \mu \mathrm{m}$ & $1.4 \mu \mathrm{m}$ \\
\hline \multirow{4}{*}{ Oxide Patch } & $\delta n_{\mathrm{TE}} / \delta n_{\mathrm{TM}}$ & -0.67 & -0.75 & -0.75 & -0.72 \\
\hline & $\sigma_{\text {film }}$ & $-330 \mathrm{MPa}$ & $-330 \mathrm{MPa}$ & $-330 \mathrm{MPa}$ & $-330 \mathrm{MPa}$ \\
\hline & Thickness (t) & $1 \mu \mathrm{m}$ & $1 \mu \mathrm{m}$ & $1 \mu \mathrm{m}$ & $1 \mu \mathrm{m}$ \\
\hline & Length (L) & $600 \mu \mathrm{m}$ & $686 \mu \mathrm{m}$ & $1057 \mu \mathrm{m}$ & $816 \mu \mathrm{m}$ \\
\hline \multirow{2}{*}{ Outputs } & TE extinction ratio & $-11 \mathrm{~dB}$ & $-40 \mathrm{~dB}$ & $-40 \mathrm{~dB}$ & $-40 \mathrm{~dB}$ \\
\hline & TM extinction ratio & $-40 \mathrm{~dB}$ & $-21 \mathrm{~dB}$ & $-20 \mathrm{~dB}$ & $-15 d B$ \\
\hline
\end{tabular}

Table 6.2: A list of device design parameters and calculated splitting ratio of the MZI-based polarization splitters and filters. 


\subsection{Photomask Layout}

After defining the device parameters, a photomask including test structures was drawn using a commercial software, AutoCad ${ }^{\circledR}$. The test devices include designs with the nominal dimensions and layout, as well as a wide range of variations to accommodate inaccuracies in as-processed feature dimensions, oxide thickness and stress. For example, the bend radii of the curved waveguides ranges from 1000 to $8000 \mu \mathrm{m}$ in the layout of the fan-in/fan-out waveguides of the $3 \mathrm{~dB}$ couplers. Variations of up to $\pm 5 \%$ in the length of the MMI couplers were also introduced to the device layout. In addition, several oxide patches with a $\pm 30 \%$ variation in length increment $\Delta L$ were added to devices with identical layout in the optical mask. The MZI devices are roughly $2.5 \mathrm{~mm} \times 16 \mu \mathrm{m}$ in size.

Figs. 6.13 shows the final design layout of the test structures. There are three types of layers in the photomask: the ridge layer (labeled as " $R$ "), the oxide cladding layer (labeled as "O"), and the edge bead layer which marks the outer boundary of the chip. Two designs of the "R" and "O" layers are shown, for the MZI devices with the etch depth $D=1.2$ and $1.4 \mu \mathrm{m}$, respectively. These layers are all "light field", meaning the digitized data enclosed by the defined boundaries are chrome (for protection), and data outside the boundaries are clear (for exposure). The photomask was written by E-beam lithography, provided by AdTek Photomask Inc. 


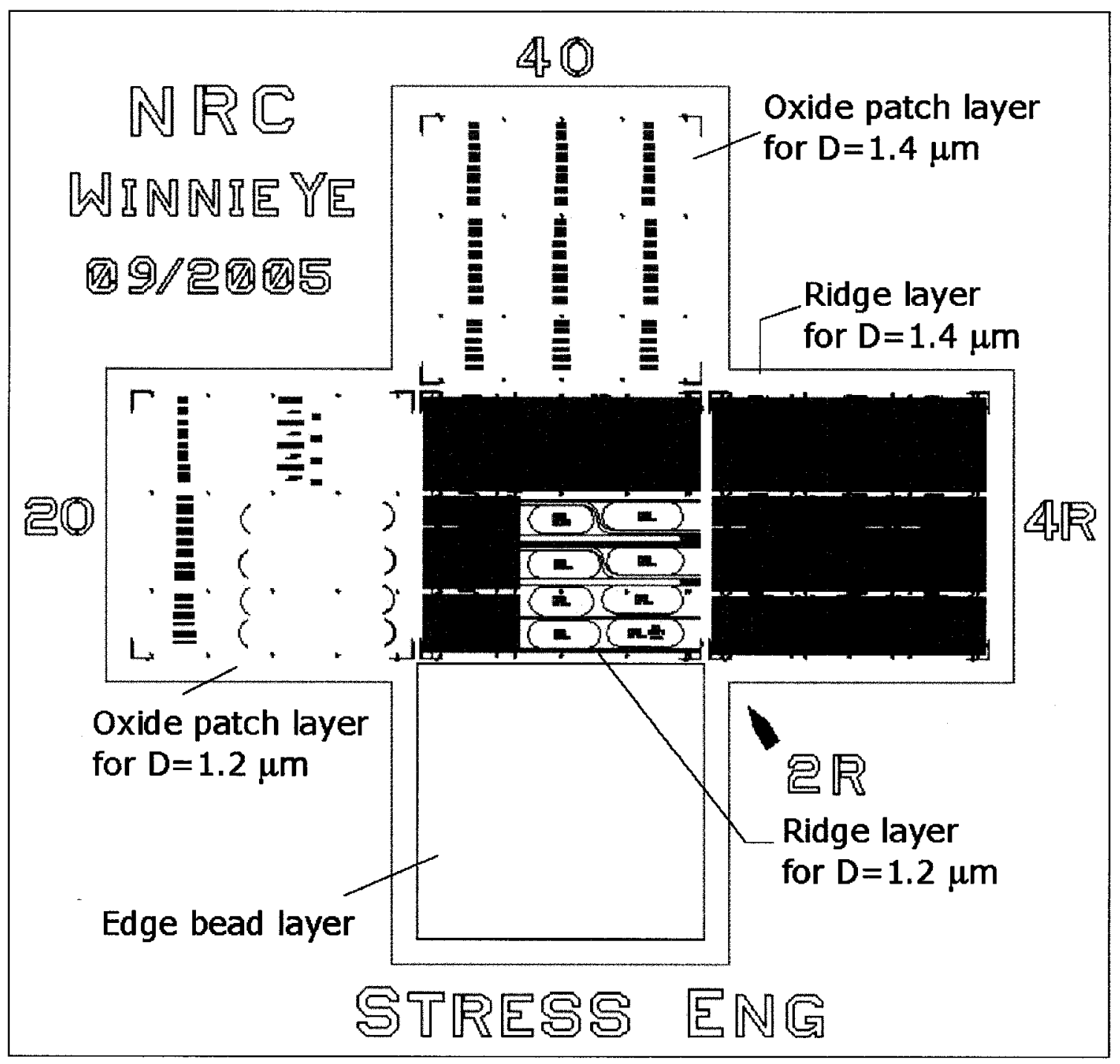

Figure 6.13: Optical masks of Mach-Zehnder interferometer based polarization test devices. The mask layout also includes ring resonator devices which are not discussed in this thesis. The typical MZI devices are roughly $2.5 \mathrm{~mm} \times 16 \mu \mathrm{m}$ in size. 


\subsection{Fabrication}

Test devices were fabricated by the Nanofabrication group at the National Research Council of Canada (NRC) and the Canadian Photonics Fabrication Center (CPFC), coordinated by Drs. P. Waldron and J. Caballero, using standard silicon processing techniques. The ridge waveguides were first patterned by photolithography, using the reactive ion etching (RIE) through the ridge mask layer "R", followed by a plasma-enhanced chemical vapor deposition (PECVD) of the silicon dioxide $\left(\mathrm{SiO}_{2}\right)$ upper cladding film over the entire chip at $\sim 400^{\circ} \mathrm{C}$. The oxide film deposition conditions were calibrated such that the thickness and the stress level in the film would produce the required polarization dependent phase shifts. The deposited oxide cladding film had a thickness of $1.0 \mu \mathrm{m}$ with a compressive stress of $\sigma_{\text {film }}=-330 \pm 15 \mathrm{MPa}$. The cladding was then patterned to the desired patch length through the oxide mask layer " $O$ ". Due to the small feature size of the oxide patch for the MZI-based devices, a dry-etching procedure was necessary to produce useable cladding on the MZI arms.

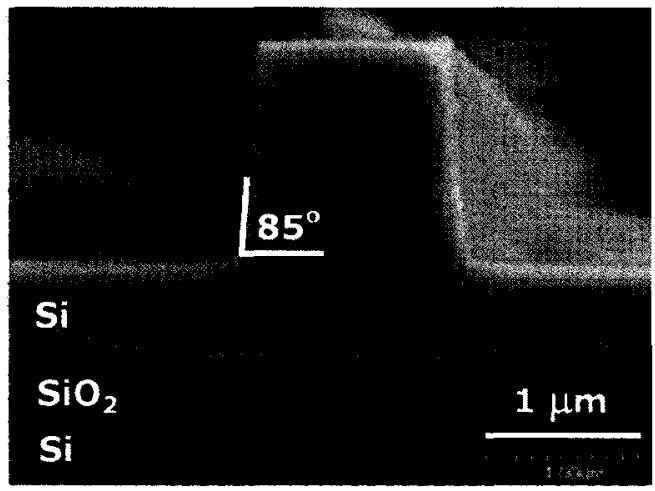

(a)

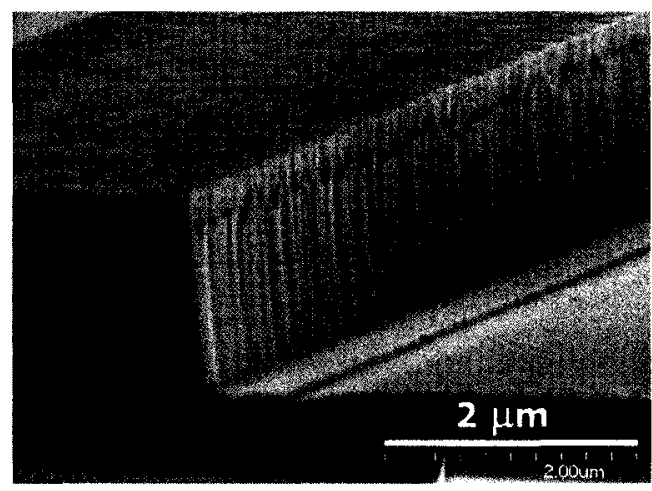

(b)

Figure 6.14: (SEM images of fabricated dry-etched ridge waveguides: (a) cross-section of a ridge waveguide; (b) sidewall roughness along an etched ridge.

SEM images of the fabricated ridge waveguides are shown in Fig. 6.14(a) and 6.14(b). The dry-etched ridge waveguides have consistent sidewall angles of $\sim 85^{\circ}$. As displayed in Fig. 6.14(b), the dry-etching method left considerable sidewall roughness (of approxi- 
mately $100 \mathrm{~nm}$ ). Propagation losses are expected to be high as a result of this sidewall roughness. Fig. 6.15 shows the optical microscope plan view images of the $3 \mathrm{~dB}$ couplers and a center section of a fabricated MZI device. The close-up images of the Y-split region and the waveguides/slab junction of an MMI are showing in Fig. 6.16. The surface roughness of the sidewalls is evident from the high-resolution SEM images of all the dry-etched MZI devices.

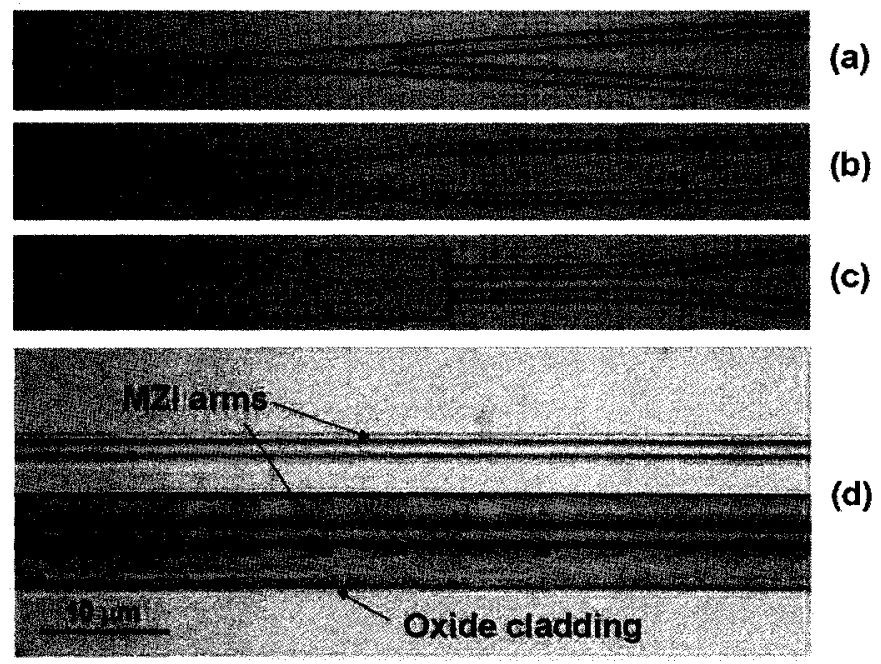

Figure 6.15: Microscope plan view of the fabricated MZI-based polarization filters/splitters: (a) a Y-junction, (b) a directional coupler, (c) an MMI coupler, (d) a deposited oxide patch on the bottom MZI arm.

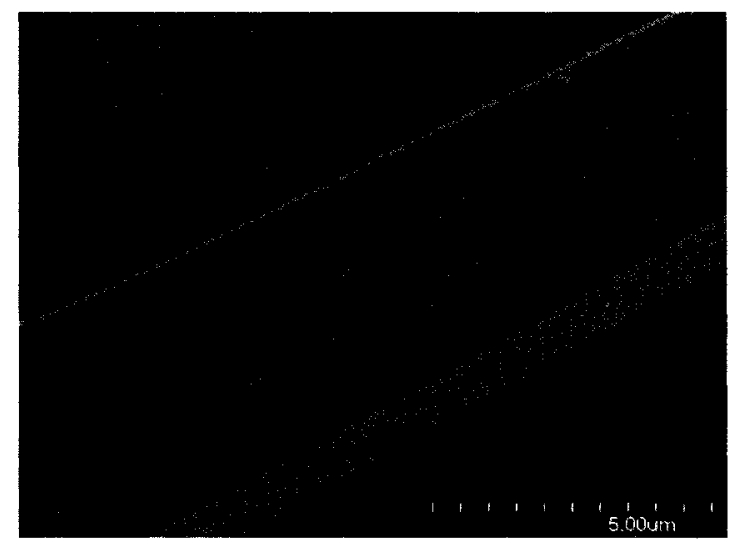

(a)

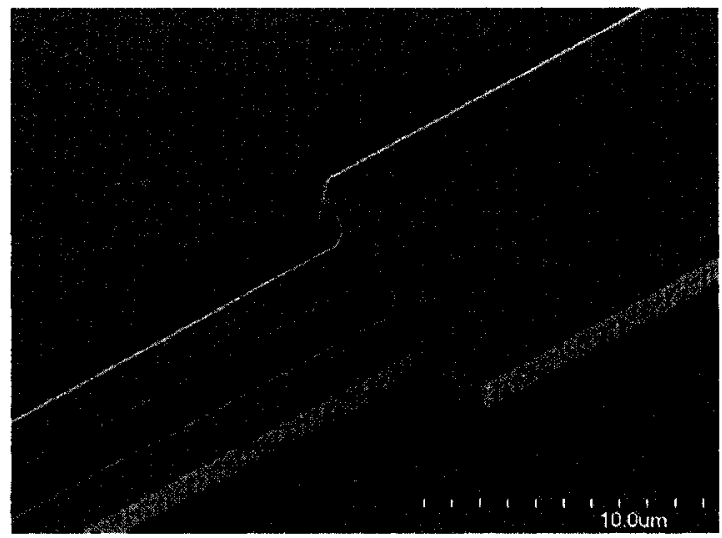

(b)

Figure 6.16: Close-up image of (a) the split region of a Y-junction and (b) the ridge waveguides/slab junction of an MMI coupler. 
The high-resolution SEM images also reveal an interesting observation of the inconsistent sidewall coverage of the oxide cladding. Fig. 6.17 suggests that regions of the ridge sidewalls have no cladding coverage. Since the stress induced from the oxide cladding on the ridge sidewalls is the main mechanism behind the polarization splitters/filters, the inconsistent sidewall cladding coverage will affect the splitting performance.

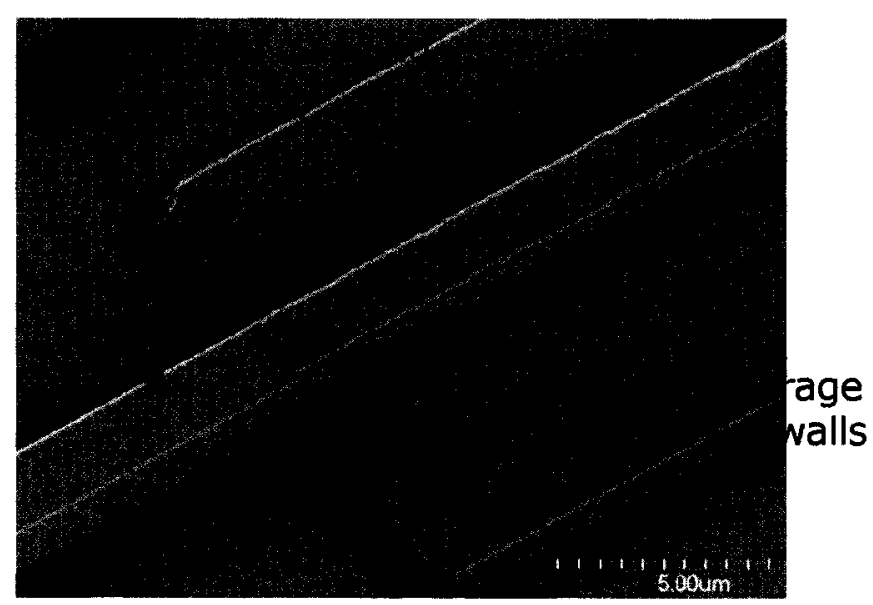

Figure 6.17: An SEM image of the oxide cladding on one MZI arm.

\subsection{Optical Measurements}

This section presents an experimental demonstration of stress-induced MZI-based polarization splitters.

\subsubsection{Experimental Setup}

Fig. 6.18 shows a picture of the testing setup used to characterize the fabricated splitter devices based on the MZI configurations. A tunable laser, with a wavelength range between $1460 \mathrm{~nm}$ to $1580 \mathrm{~nm}$, was coupled into a polarization-maintaining (PM) fiber. The laser beam went through an in-line polarization rotator such that the input beam could be polarized in either the TE or the TM direction. The beam was then focused onto the input facet of the test device by a single-mode (PM) fiber with a tapered tip. The 


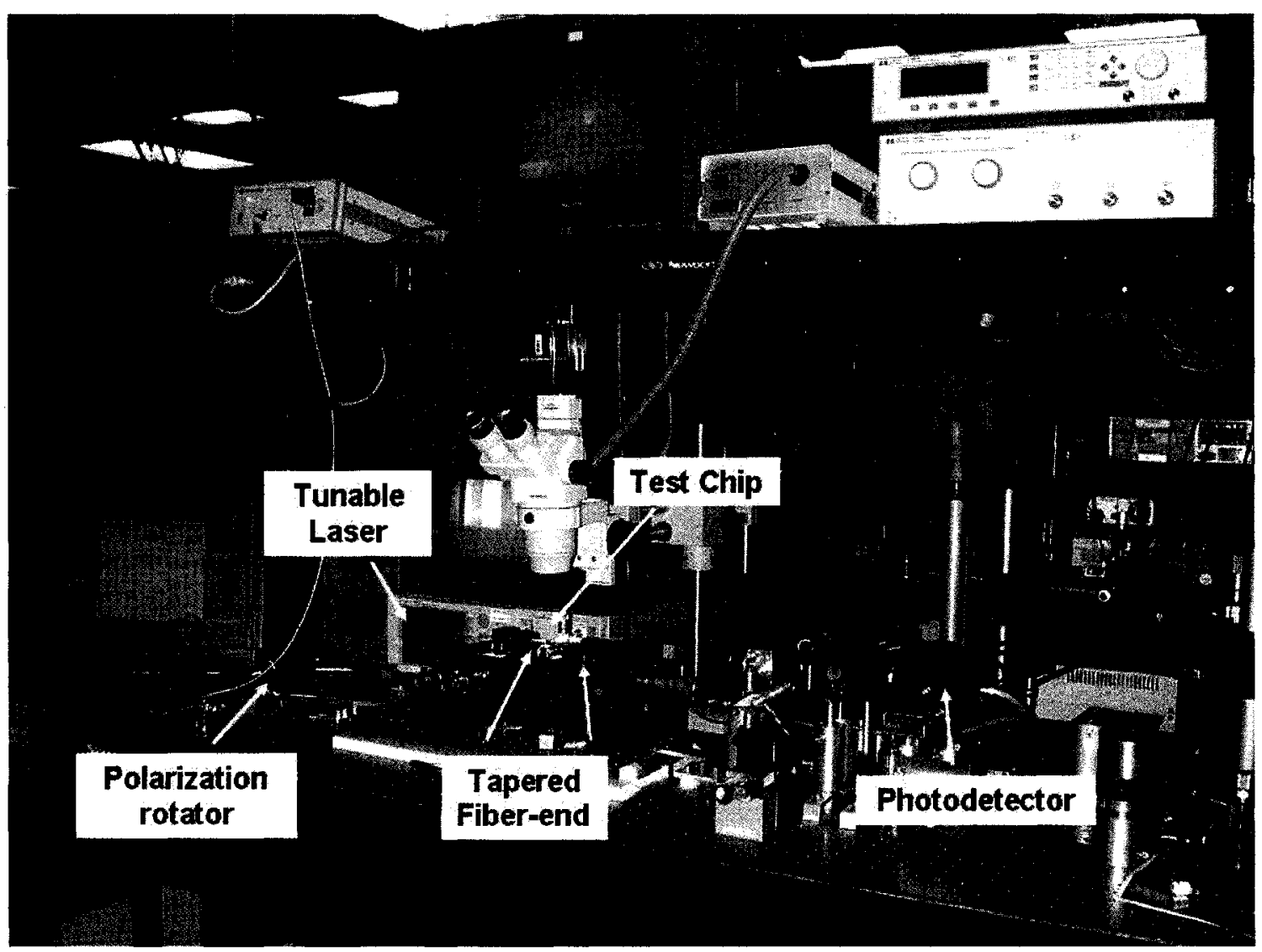

Figure 6.18: A picture of the testing stage for the waveguide polarization splitter measurements. A tapered single-mode fiber is used to collect the device output power.

diameter of the fiber tip is $\sim 2 \mu \mathrm{m}$, which matches well with the mode size of the input waveguide. Light passed through the device and was then collected by another tapered single-mode fiber, whose output power was read by the photodetector. This testing stage is similar to the setup described for the AWG demultiplexer in Fig. 5.4, except the device output was collected by a tapered single-mode fiber instead of an objective lens. This is a useful modification which reduces the effect of stray light and higher-order modes (real or leaky) in the polarization splitter outputs. With the proper alignment of the output single-mode fiber, the measured power should be mainly from the fundamental waveguide mode. 


\subsubsection{Test Results}

Fig. 6.19 presents the full wavelength scans of two MZI-based polarization splitters: one

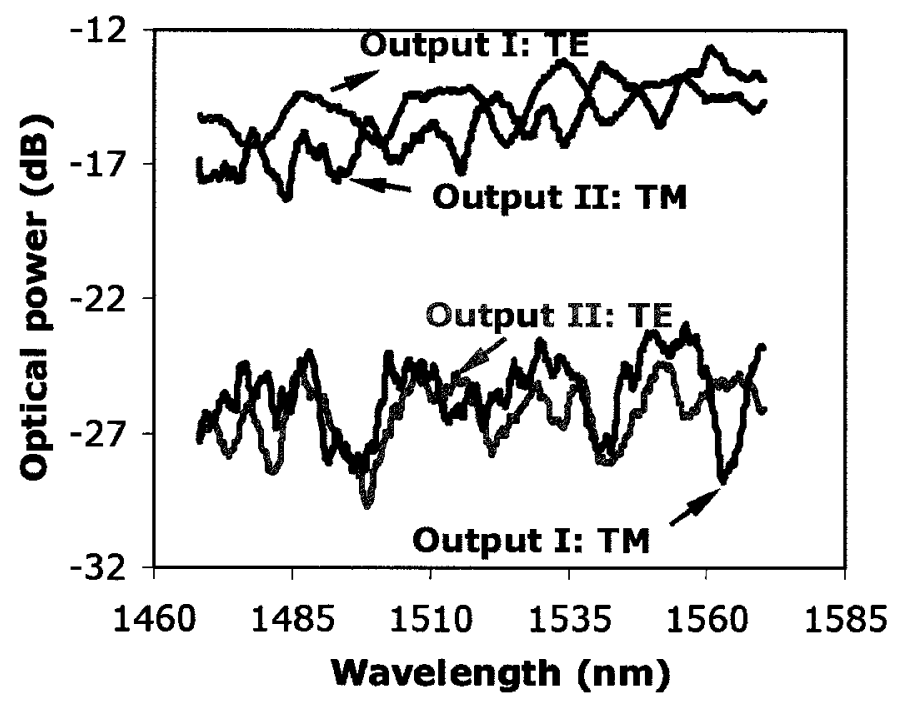

(a)

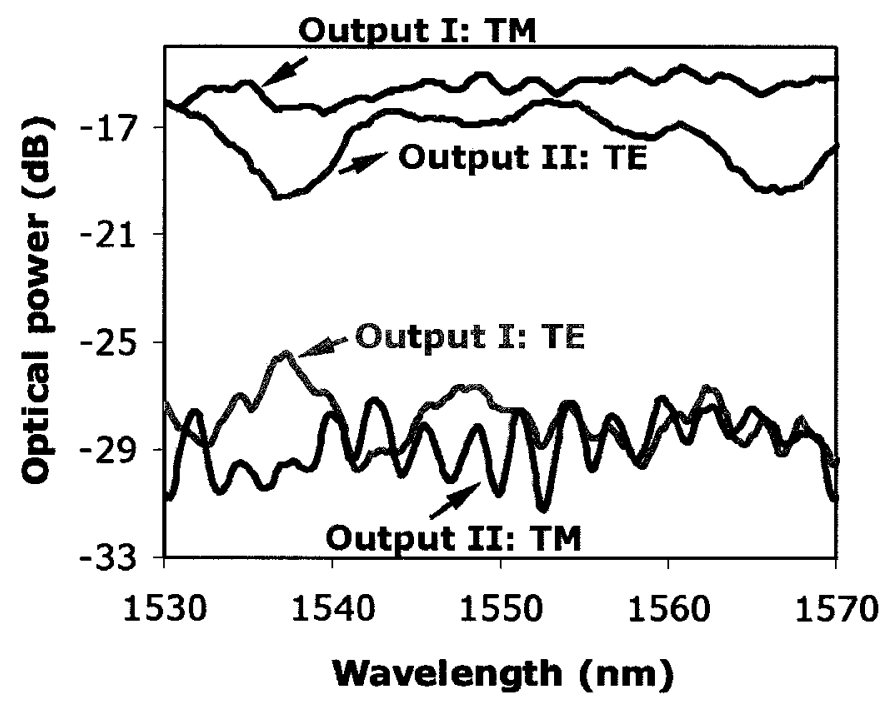

(b)

Figure 6.19: Broadband MZI-based polarization splitters: (a) MZI with asymmetric $3 \mathrm{~dB}$ couplers (i.e., one Y-junction + one MMI); (b) MZI with two symmetric MMI couplers. The ridge waveguides for both devices have the following dimensions: $W=1.5 \mu \mathrm{m}, D=1.4 \mu \mathrm{m}$, oxide cladding is under a compressive film stress $\sigma_{\text {film }}=-345 \mathrm{MPa}$, with a length $L=800 \mu \mathrm{m}$ and a thickness $t=1.2 \mu \mathrm{m}$. The MMIs have $W_{\mathrm{MMI}}=10.5 \mu \mathrm{m}$ and $L_{\mathrm{MMI}}=$ $167 \mu \mathrm{m}$. 
asymmetric type (i.e., with one $\mathrm{Y}$-junction and one MMI), and one symmetric type (i.e., with two MMIs). An oxide cladding with a measured thickness of $t=1.2 \mu \mathrm{m}$ and a compressive stress of $\sigma_{\text {film }}=-345 \mathrm{MPa}$, was deposited to provide the required polarization dependent phase shift. Both as-designed waveguide devices have a nominal ridge width of $W=1.5 \mu \mathrm{m}$ and an etch depth of $D=1.4 \mu \mathrm{m}$. However, SEM images of the ridge cross-section geometry suggest that etch depth is almost $0.2 \mu \mathrm{m}$ deeper than nominal, with $D \approx 1.6 \mu \mathrm{m}$. This means that the waveguides may support higher-order modes. The presence of the higher-order modes may be the source of large intensity ripples, evident in all wavelength scans as illustrated in Fig. 6.19. Although MZI with Y-junctions and MMIs showed acceptable performance, none of the polarization splitters with directional couplers worked, possibly a result of higher-order mode generation and coupling in the directional couplers.

The intensity profiles presented in Figs. 6.19(a) and 6.19(b) demonstrate the broadband performance of the MZI-based polarization splitters with MMIs. An average splitting extinction ratio of $-10 \mathrm{~dB}$ has been observed for both polarizations over a wide wavelength range. However, we have not successfully observed the $-20 \mathrm{~dB}$ extinction ratio predicted by the simulations shown in Fig. 6.12. The degraded splitting performance may be a result of the poor cladding coverage on the ridge sidewalls (shown in Fig. 6.17). Simulations were carried out to characterize the stress-induced index change with and without cladding coverage on waveguide sidewalls. Although the average $\sigma_{\text {film }}$ value of the cladding film with and without cladding remains at $-330 \mathrm{MPa}$, Fig. 6.20 illustrates the different stress distribution in the vicinity of the ridge waveguide due to the sidewall cladding coverage. The altered stress distribution in turn modifies the stress-induced index ratio $\frac{\partial n_{\mathrm{TE}}}{\partial n_{\mathrm{TM}}}$ from -0.68 to -11.7 . Consequently, the expected $-28 \mathrm{~dB}$ polarization splitting extinction ratio for both polarizations are changed to $-6 \mathrm{~dB}$ for the TE mode and $-1 \mathrm{~dB}$ for the TM. These simulation results are based on the worst-case scenario with no sidewall cladding coverage over the entire patch region. Although the 


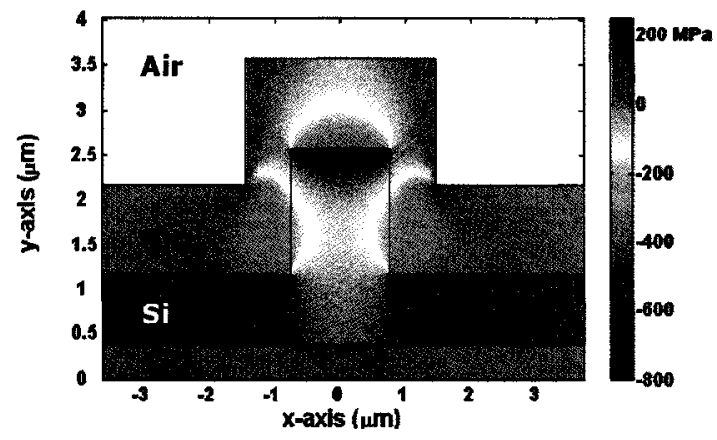

(a)

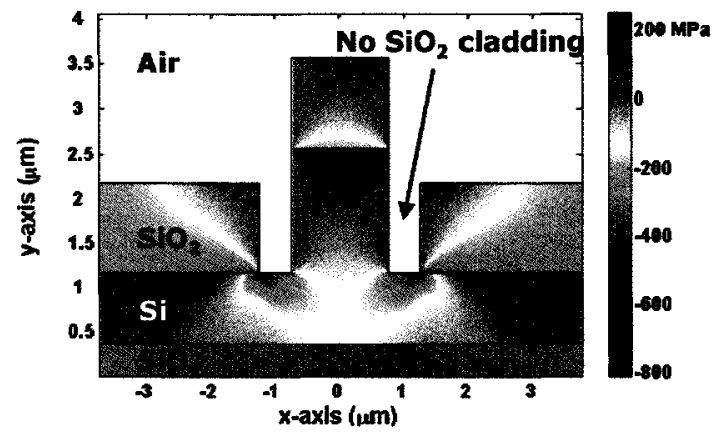

(b)

Figure 6.20: Simulated stress distribution for ridge waveguides. The oxide cladding has (a) full sidewall coverage and (b) has zero sidewall coverage. The dimensions of the ridge waveguide are: $W=1.5 \mu \mathrm{m}, H=2.2 \mu \mathrm{m}$, $D=1.4 \mu \mathrm{m}$ and $t=1 \mu \mathrm{m}$.

simulations overestimate the impact of the sidewall cladding in the device performance, the sidewall cladding may be the main source of the degrading splitting performance. Other factors such as the excitation of the higher-order modes in the ridge waveguides, the dimensional variations in fabricated waveguides and the sidewall roughness may also affect the device performance. Since this is our first attempt in fabricating these devices, improved fabrication techniques, optimized calibration of the etch and oxide deposition recipes, and better designs of the directional couplers could address some of the critical issues and enhance the device performance.

\subsection{Summary}

This chapter presented the operating principles of novel stress-induced polarization splitters/filters based on various MZI configurations. The polarization splitting/filtering function was realized by adjusting the cladding stress induced polarization dependent phase shifts. The critical design parameters such as the polarization splitting/filtering phase relations and the oxide patch lengths were analyzed and specified. The experimentally demonstrated MZI-based polarization splitters/filters exhibited a broadband polarization 
splitting with an extinction ratio better than $-10 \mathrm{~dB}$ for both output polarizations over wavelengths ranging from $1460 \mathrm{~nm}$ to $1570 \mathrm{~nm}$. 


\section{Chapter 7}

\section{Zero-order Arrayed Waveguide Grating Based Polarization Splitters}

The previous chapter described the design and characterization of the MZI-based polarization splitters/filters. This chapter deals with a different design of the passive polarization splitters using stress engineering. The new design adopts a zero-order arrayed waveguide grating (AWG) configuration. The actual design, fabrication, and characterization of the zero-order AWG devices are investigated in detail.

\subsection{Introduction}

AWGs were first proposed by Smit et al. in 1988 [94]. A typical AWG layout is shown in Fig. 7.1 [94]. The device consists of an input waveguide and a number of output waveguides, both coupled to a waveguide array through two slab regions (called the "free propagation regions" or FPRs). The input beam which may consist of a number of wavelength channels is coupled into a single input waveguide. As the input light enters the FPR region from the left, it is no longer laterally confined and diverges. This FPR is sometimes referred to as the splitter region. Upon arriving at the array input aperture, the beam is coupled into all the individual arrayed waveguides and propagates towards 


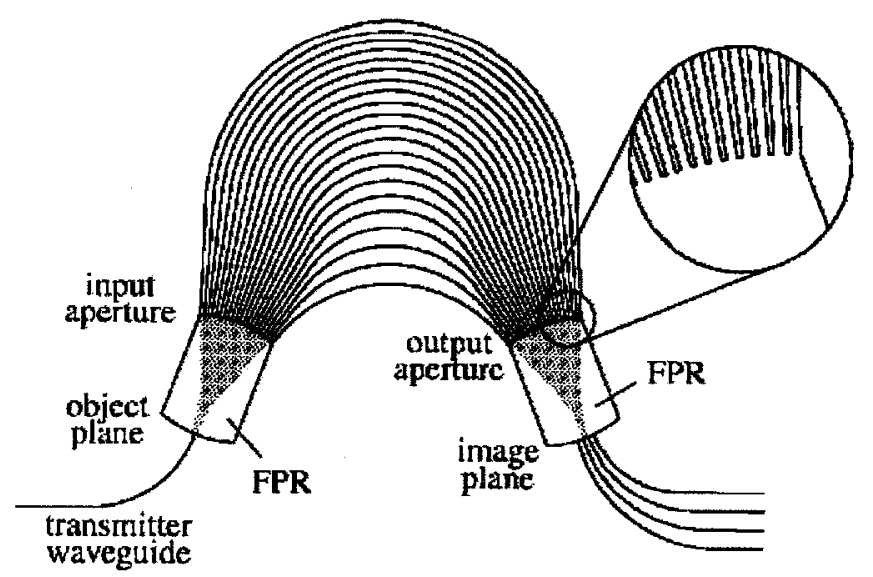

(a)

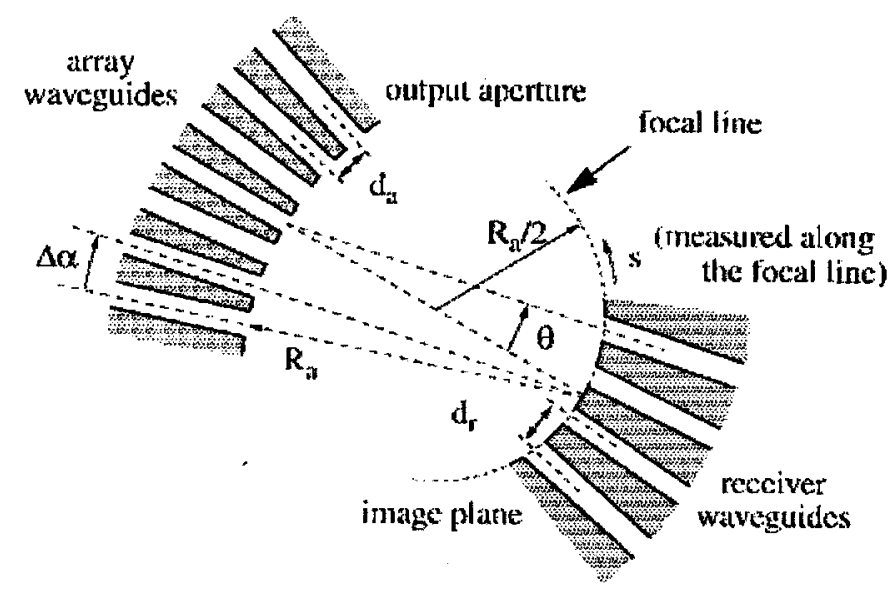

(b)

Figure 7.1: (a) Layout of a typical arrayed waveguide grating (AWG) structure; (b) Geometry of the receiver free-propagation region. From [94].

the output aperture. The radius of the input aperture is chosen to match the length of the FPR, such that the waveguides in the array are excited with the same phase. The design parameters of the FPR region are shown in Fig. 7.1(b).

The phase difference between light exiting two adjacent arrayed waveguides is

$$
\Delta \phi=\beta \Delta L=\frac{2 \pi}{\lambda} n_{\mathrm{eff}} \Delta L
$$

where $\beta$ and $n_{\text {eff }}$ are the propagation constant and the refractive index of the waveguide mode, respectively, $\lambda_{0}$ is the central wavelength, and $\Delta L$ is the length difference between 
the two adjacent array waveguides. Note that this length difference is the same between any two adjacent waveguides in the array. $\Delta L$ is required to be an integer multiple of the center wavelength $\left(\lambda_{0}\right)$ to ensure the outgoing beams are in phase (modulo of $2 \pi$ ) at the output aperture. For example, a phase difference of $\Delta \phi=2 M \pi$ (where the grating order $M$ is an integer) is experienced by the center wavelength channel $\lambda_{0}$. Other wavelength channels from the input will experience different phase shifts while traveling through the arrayed waveguides.

The output FPR is identical to the splitter FPR region, except it acts as a combiner, transforming the field distributions at the output aperture into one convergent beam at the focal point on the image plane. At the center wavelength $\lambda_{0}$, the output of this channel is focused to the center output waveguide. The focal points of the outgoing beams of other wavelength channels are shifted along the image plane, away from the center output waveguide. By placing receiver waveguides at proper positions along the image plane, spatial separation of the different wavelength channels can be obtained. This AWG acts as a wavelength demultiplexer or spectrometer.

Since the phase shift in waveguides depends on the polarization as well as the operating wavelength, a phased array can also operate as a polarization splitter. In Chapter 2, the splitter design based on an AWG configuration was illustrated in Fig. 2.17. The polarization splitter device is simply a spectrometer with a strong polarization dependent wavelength shift. It has one significant performance limitation: wavelength dependent performance.

\subsection{Splitter Operating Principles}

Recently, Flück et al. [95] suggested the use of a waveguide array in a wavelength independent beam switch. Although the device does not function as a polarization splitter, it is worth mentioning the mechanism behind its wavelength independent operation. The 
geometrical layout of this waveguide array is illustrated in Fig. 7.2. Unlike the AWGs

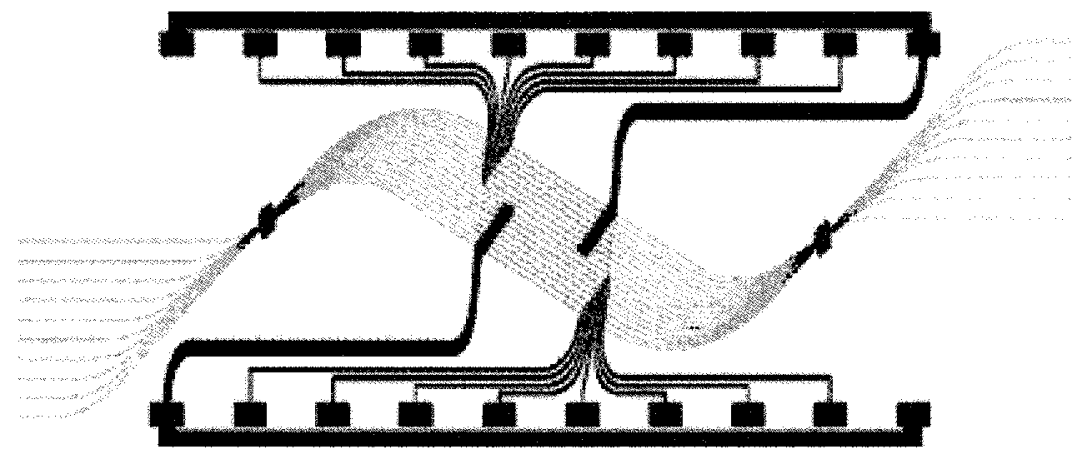

Figure 7.2: Geometrical layout of a waveguide array based thermo-optical switch, with the optical waveguides, chromium heaters, and aluminum leads. From [95].

mentioned earlier, all the waveguides in the arrayed section of the device have equal lengths $(\Delta L=0)$ and are equipped with individual tunable heaters, which function as phase shifters. We can call this device a "zero-order" AWG since the diffraction order is zero (i.e., $m=\frac{n_{\text {eff }} \Delta L}{\lambda_{0}}=0$ ) when no power is applied to the heater. Thus the output beam remains stationary as wavelength is scanned. The phase distribution across the arrayed waveguides can be adjusted thermal-optically with the tunable heaters, thus enabling the beam in the output coupler to be directed to any one of the output channel. Even though the device was fabricated in a high-index-contrast silicon-oxynitride ( $\mathrm{SiON}$ ) system, this 1-to- 8 beam steering device with only 16 arrayed waveguides has a dimension of $33.5 \mathrm{~mm} \times 8 \mathrm{~mm}$, including the tunable heaters and their electrical pads [95]. The mechanism behind the wavelength independent switching in this device is simple; however, the one heater per waveguide scheme makes it unmanageable when more arrayed waveguides are required to optimize the device performance.

In order to achieve wavelength independent polarization splitting, we begin by adopting the zero-order AWG structure, with identical channel length for all the waveguides in the arrayed grating section (i.e., $\Delta L=0$ ). The operating principles of the proposed device are similar to a regular AWG described in Fig. 7.1, except the phase shifts are polarization dependent rather than wavelength dependent. 
Fig. 7.3 presents a schematic diagram of a zero-order AWG with 100 arrayed ridge waveguides. A patch of oxide cladding is selectively deposited in the arrayed waveguide section (the triangular patch in Fig. 7.3) to serve as the phase shifter. The stress in the waveguide cladding induces a polarization dependent phase difference in the light beams propagating in the arrayed grating section. Since each waveguide in the arrayed section has the same length, the phase change across the arrayed section is solely dependent on the applied stress. Thus, Eq. 7.1 is transformed into an expression similar to Eq. 6.2. The stress-induced phase difference $(\Delta \phi)$ between two adjacent arrayed waveguides for each polarization is

$$
\Delta \phi^{\mathrm{TE}}=\frac{2 \pi}{\lambda_{0}} L \delta n_{\mathrm{TE}}, \quad \Delta \phi^{\mathrm{TM}}=\frac{2 \pi}{\lambda_{0}} L \delta n_{\mathrm{TM}},
$$

respectively. Here $L$ is the length increment of the cladding patch between two adjacent waveguides in the arrayed section, and $\delta n_{\mathrm{TE}}$ and $\delta n_{\mathrm{TM}}$ are the stress-induced effective index change in the TE and TM polarization, respectively.

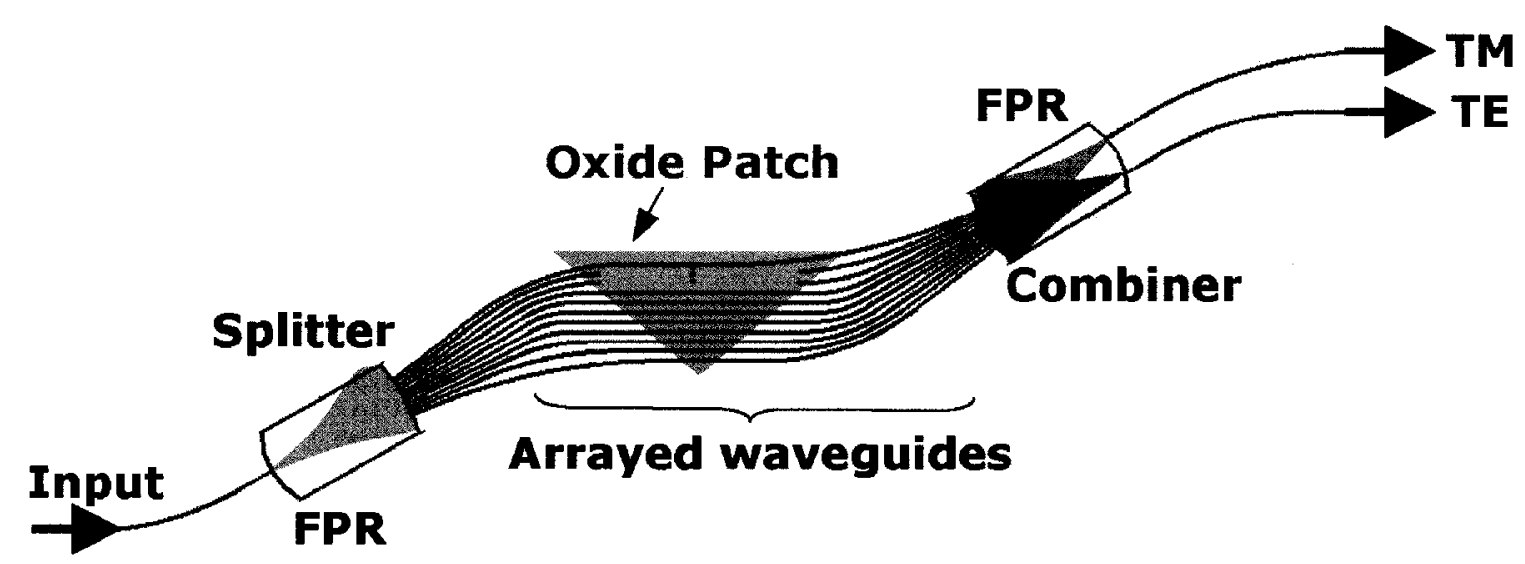

Figure 7.3: A schematic diagram of a zero-order arrayed waveguide grating. The waveguides in the arrayed section have identical channel length. The green enclosed area indicates the size of the selectively deposited oxide cladding patch.

Each polarization experiences a unique stress-induced phase shift, resulting in separated focal points of the TE- and TM-polarized beams at the output FPR region. In other words, there is stress-induced lateral separation between the focal points of the 
two polarizations. By placing the output waveguides at proper positions along the image plane of the output FPR, spatial separation or splitting of the TE and TM polarizations can be obtained. Since the AWG is always close to zero-order even when stress is applied, the device is approximately wavelength independent over a wide bandwidth. The achievable polarization extinction ratio is limited by the AWG crosstalk, which can be better than $-40 \mathrm{~dB}$ in commercial devices.

\subsection{Design Considerations}

\section{Waveguide Cross-section And Bends}

We follow similar design rules described in Chapter 6.3.1 for designing the cross-sectional geometry of the waveguides involved in the zero-order AWGs. Two widths have been chosen for the ridge waveguides: $1.5 \mu \mathrm{m}$ and $2.0 \mu \mathrm{m}$. It is evident from Fig. 4.15 that the relative contribution of the stress-induced effects on total birefringence are the strongest at $D=1.5 \mu \mathrm{m}$ for a ridge core with a $2.2 \mu \mathrm{m}$ thickness. Thus, the etch depth of $D=$ $1.5 \mu \mathrm{m}$ is used in the design of the AWG waveguides to ensure optimal cladding induced stress for polarization compensation and to allow tighter waveguide mode confinement to provide reduced overall device sizes.

A ridge waveguide with a deep etch depth means that the waveguide no longer resides in the single mode regime as stated in Eq. 1.6, and the waveguide is expected to be multimode. However, since the higher-order waveguide modes experience a much larger radiation loss than the fundamental mode at a given bend radius [31], it is possible to "filter out" the undesired higher-order modes by using curved waveguides with an appropriate bending radius. Curved waveguides are implemented in our design here. Fig. 7.4 illustrates the bending losses calculated for the ridge waveguides with an etch depth and width of $D=W=1.5 \mu \mathrm{m}$. These calculations are obtained from an optical waveguide mode solver OWMS by Apollo Photonics. The calculated bending loss as 
a function of the radius of curvature demonstrates a sharp threshold. Above a radius of curvature of $\sim 200 \mu \mathrm{m}$ effectively no loss in the fundamental TE and TM modes is observed. Fig. 7.4 also illustrates that the threshold for bending loss is dependent on the waveguide mode. Higher-order modes leak away at larger radii of curvature. For example, the first-order TE and TM modes have a threshold radius of $600 \mu \mathrm{m}$ and 250 $\mu \mathrm{m}$, respectively. The minimum bending radius of the arrayed waveguides is designed to

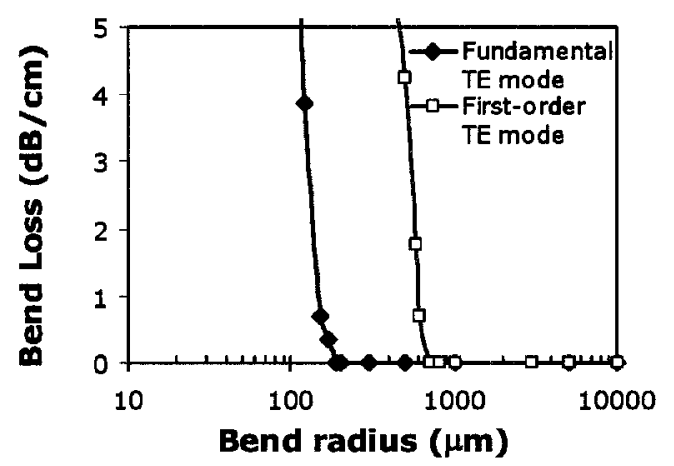

(a)

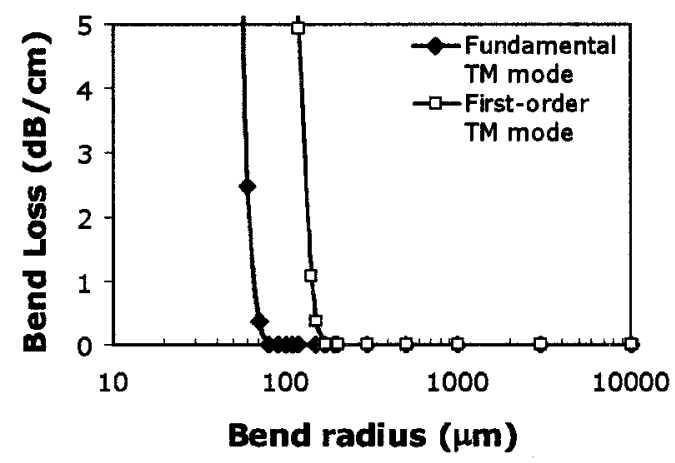

(b)

Figure 7.4: Calculated bending loss vs. radius of curvature for ridge waveguides with $W=D=1.5 \mu \mathrm{m}$ and $H=2.2 \mu \mathrm{m}$ : (a) fundamental and firstorder TE modes and (b) fundamental and first-order TM modes.

be $R_{\min }=500 \mu \mathrm{m}$, which is much higher than the calculated threshold radius for the fundamental mode, and lower than the threshold radius for the higher-order modes.

\section{Free Propagation Region Design}

The most critical design part of a zero-order AWG is the free propagation regions (FPRs), which provide the light splitting and combining functions. Fig. 7.5 illustrates the geometry of an output FPR region. It is noted that we use an identical FPR design for the input combiner FPR. The FPRs in an AWG are typical examples of Rowland mountings [94]. The focal line of the mounting, which defines the image plane, follows a circle with a radius of $R_{a} / 2$ as shown in Fig. 7.5. Here $R_{a}$ is the distance from the center of the image plane to the array waveguides, and is usually referred to as the length of the FPR. To determine the angular position where different polarization (TE or TM) focuses 


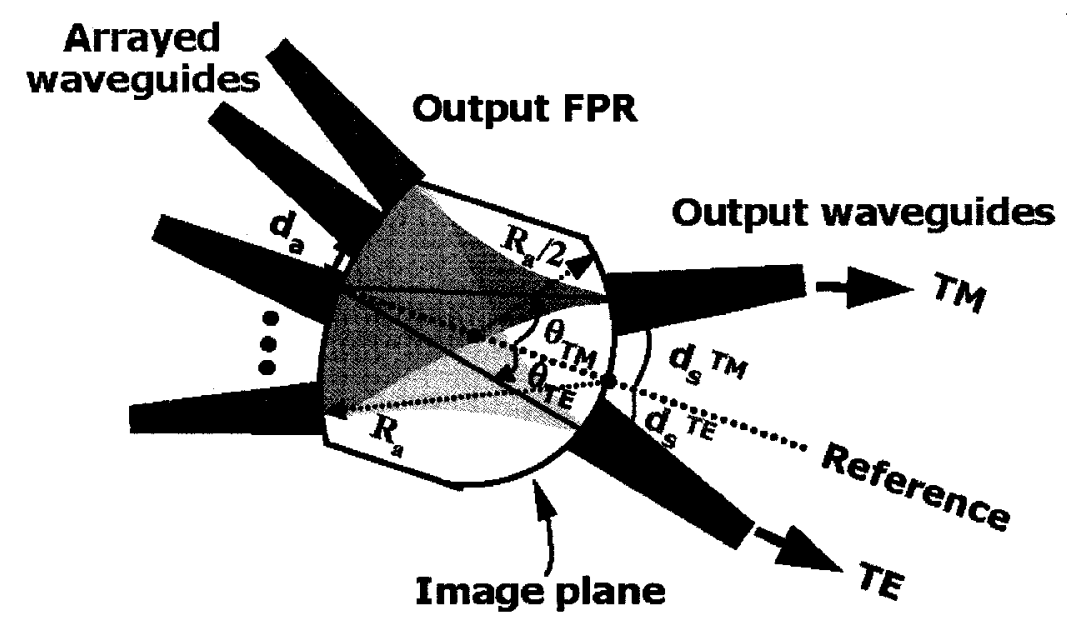

Figure 7.5: The geometry of the output side free-propagation region (FPR) of a zero-order AWG device. The output aperture and the image plane follow a typical design of a Rowland mounting.

at the image plane, we need to obtain the angle $\theta$ of the diffracted beam resulting from a stress-induced phase difference $\Delta \phi$. Referring to Fig. 7.5,

$$
\theta=\sin ^{-1}\left(\frac{\Delta \phi-2 m \pi}{\beta_{s} d_{a}}\right)=\sin ^{-1}\left(\frac{\lambda \Delta \phi}{2 \pi n_{s} d_{a}}\right) \quad(\mathrm{m}=0 \text { for zero order })
$$

where $\Delta \phi$ is the stress-induced phase difference which is different depending on the polarization, $\beta_{s}$ and $n_{s}$ are the propagation constant and the refractive index of the FPR slab region, respectively, and $d_{a}$ is the center to center spacing of the arrayed waveguides at the aperture of the FPR. By substituting the expression of $\Delta \phi$ from Eq. 7.2 into Eq. 7.3, we obtain the diffraction angle for the TE and TM polarizated light,

$$
\theta_{\mathrm{TE}}=\sin ^{-1}\left(\frac{L \delta n_{\mathrm{TE}}}{d_{a} n_{s}^{\mathrm{TE}}}\right) \quad \text { for } \mathrm{TE} ; \quad \theta_{\mathrm{TM}}=\sin ^{-1}\left(\frac{L \delta n_{\mathrm{TM}}}{d_{a} n_{s}^{\mathrm{TM}}}\right) \quad \text { for } \mathrm{TM}
$$

Since the stress-induced index change in the TE and TM polarization modes are opposite in sign (see Fig. 4.11), the diffraction angles for the two modes are in opposite directions from the reference centerline, as shown in Fig. 7.5. The required spatial separation $d_{s}$ between the TE and TM output ports can be calculated from the angular separation (Eq. 7.4) in the output FPR region for each polarization mode as follows

$$
\begin{gathered}
d_{s}^{\mathrm{TE}}=R_{a} \sin \theta_{\mathrm{TE}} \quad \text { for } \mathrm{TE} ; \quad d_{s}^{\mathrm{TM}}=R_{a} \sin \theta_{\mathrm{TM}} \quad \text { for } \mathrm{TM} \\
d_{s}(\text { total })=\left|d_{s}^{\mathrm{TE}}\right|+\left|d_{s}^{\mathrm{TM}}\right|=\frac{R_{a} L}{d_{a}}\left(-\frac{\delta n_{\mathrm{TE}}}{n_{s}^{\mathrm{TE}}}+\frac{\delta n_{\mathrm{TM}}}{n_{s}^{\mathrm{TM}}}\right) .
\end{gathered}
$$


It can be seen that the operating wavelength $\lambda$ is not an important design parameter in the FPR and the overall AWG design. Compared to the MZI-based devices discussed in Chapter 6, the main advantages of the zero-order AWG devices include the wavelength independent polarization splitting operation and the ease of fabrication. Although a perfectly balanced MZI itself may not be wavelength dependent, its 3dB couplers usually are not completely insensitive to wavelength variations. In addition, the stress-induced phase shifts ( $\delta n_{\text {stress }}^{\mathrm{TE}}$ and $\delta n_{\text {stress }}^{\mathrm{TM}}$ ) are no longer required to satisfy the phase difference conditions for polarization splitting as in the case of MZI-based devices. Care only need to be taken in the lateral placements of the output waveguides to capture the TE and TM light. Furthermore, the proposed zero-order AWGs have relatively relaxed fabrication tolerances as long as the output ports are placed properly along the image plane of the FPR region.

\subsection{Simulation Results}

A true zero-order AWG without the cladding compensator is symmetrical. If light is coupled from the input side of the first FPR splitter, an identical image of the input mode is expected to be reproduced at the image plane of the output FPR combiner and appear at the corresponding output port. With the inclusion of a stressed oxide cladding patch, the two orthogonal polarizations have different stress-induced phase shifts after propagating through the array section, resulting in a lateral spatial separation between the output TE- and TM-polarized mode. The stress-induced index changes, $\delta n_{\text {stress }}^{\mathrm{TE}}$ and $\delta n_{\mathrm{stress}}^{\mathrm{TM}}$, are usually on the order of $10^{-4}$ to $10^{-3}$, which introduces a small diffraction order to the AWG. Assuming that the patch length increment between two adjacent waveguides is $\Delta L=16.2 \mu \mathrm{m}$ and the center wavelength is $\lambda_{0}=1550 \mathrm{~nm}$, the AWG would have a stress-induced order of $m=\delta n_{\text {stress }} \Delta L / \lambda_{0} \approx 0.001$ to 0.01 . Since $\delta n_{\text {stress }}$ is independent of wavelength (as discussed in Fig. 4.14), and the AWG is operating close to an zero- 
order, this splitter should be almost entirely independent of the operating wavelength, providing a ultra-broadband polarization splitting function. Fig. 7.6 illustrates the spatial separations of the output TE and TM polarizations as a function of operating wavelength. As explained earlier, the stress-induced index changes $\delta n_{\mathrm{TE}}$ and $\delta n_{\mathrm{TM}}$ are opposite in sign, thus the spatial separations $d_{s}^{\mathrm{TE}}$ and $d_{s}^{\mathrm{TM}}$ are opposite in direction, as depicted in Fig. 7.5. For this AWG device, the rate of change in the spatial separations $d_{s}^{\mathrm{TE}}$ and $d_{s}^{\mathrm{TM}}$ are both around $0.003 \mu \mathrm{m}$ per $1 \mathrm{~nm}$ change in the operating wavelength. The slight wavelength dependence is mainly due to the dispersion of the slab FPR regions.

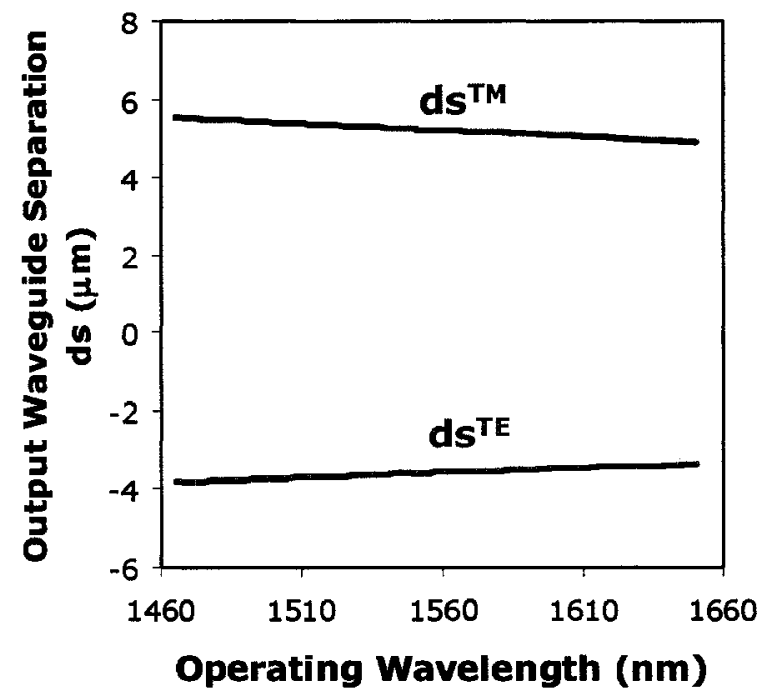

Figure 7.6: The spatial separation of the output TE and TM polarizations as a function of operation wavelength. The AWG has an oxide patch length increment $\Delta L=16.2 \mu \mathrm{m}$. The oxide cladding has a thickness of $0.8 \mu \mathrm{m}$ with a stress of $\sigma_{\text {film }}=-300 \mathrm{MPa}$. The ridge waveguides have the following dimensions: $W=D=1.5 \mu \mathrm{m}$ and $H=2.2 \mu \mathrm{m}$.

A zero-order AWG device is specified by the following characteristics: the length of the FPR region $\left(R_{a}\right)$, the total number of the arrayed waveguides $(N)$, the minimum waveguide separation at the junction between the arrayed waveguides and the FPR region $\left(d_{a}\right)$, the minimum spatial separation of the output waveguides $\left(d_{s}\right)$, and the minimum bend radius. The designs of the two free-propagation regions (FPRs) are very similar to each other. A schematic diagram of an output FPR can be found in Fig. 7.5. Fig. 7.7 shows a corresponding input FPR. The end width of the tapered input waveguide, $d_{w}$, 


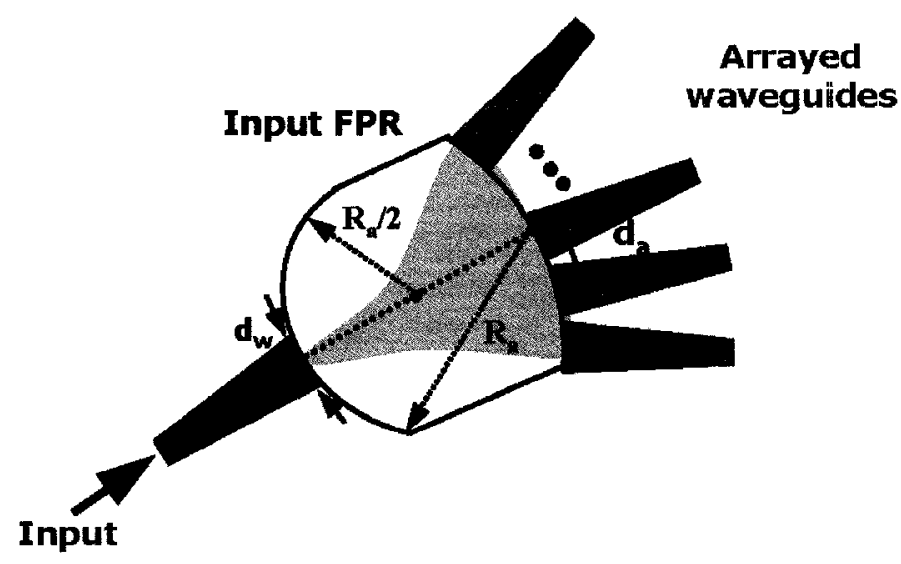

Figure 7.7: The geometry of the input free-propagation region (FPR) of a zero-order AWG device.

and the radius of the Rowland circle in the FPR region, $R_{a}$, are designed to be $3 \mu \mathrm{m}$ and $1500 \mu \mathrm{m}$, respectively, which are adopted from the AWG devices previously designed by Pearson and Cheben et al. $[7,31]$. As the input light enters the FPR region from the left, it is no longer laterally confined and becomes divergent. The electric field distribution at the output aperture of the FPR is a Fourier transform of the field distribution of the input. A $3 \mu \mathrm{m}$ input slit $\left(d_{w}\right)$ corresponds to a far field envelope width of $\frac{2.44 \lambda_{0}}{d_{w} n_{\mathrm{s}}} R_{a} \approx 540 \mu \mathrm{m}$ at the output aperture of the FPR. This width restricts the total number of the array waveguides. Ideally, the total number of arrayed waveguides should be sufficiently large to minimize the loss of light captured by the arrayed grating section. Here $N=100$ is used. The center-to-center spacing, $d_{a}$, between two adjacent arrayed waveguides is designed to be $4 \mu \mathrm{m}$, to ensure most of the divergent light is collected by the 100 arrayed waveguides. Again, the arrayed waveguides have wider tapered ends at the FRP-array boundary to reduce the insertion loss.

The output FPR in Fig. 7.5 is identical to the input FPR in Fig. 7.7, except for the arrangement of the input and output waveguides. Factors determining the placement of the output waveguides have been discussed in detail earlier in the chapter. Table 7.1 lists the required spatial separations in the output waveguides, as well as the specifications for the oxide patch length. The AWG designs for ideal, dry and wet etched ridge waveguides 
with widths of 1.5 and $2 \mu \mathrm{m}$ are included. The dimensions of the dry-and wet-etched waveguides are taken from the experimental measurements of the previously fabricated waveguides in Table 5.1.

\begin{tabular}{|c|c||c|c|c|c|}
\hline \multicolumn{2}{|c|}{ Zero-order AWG } & OxIde thickness & Oxide Length Increment & ds $^{\text {TE }}(\mu \mathrm{m})$ & ds $^{\text {Th }}(\mu \mathrm{m})$ \\
\hline Dry & $W_{1}=1.35 \mu \mathrm{m} / \mathrm{W}_{2}=1.50 \mu \mathrm{m}$ & $0.8 \mu \mathrm{m}(-300 \mathrm{MPa})$ & $\Delta \mathrm{L}=16.4 \mu \mathrm{m}$ & -3.67 & +5.15 \\
\hline Nominal & $W_{1}=1.50 \mu \mathrm{m} / \mathrm{W}_{2}=1.50 \mu \mathrm{m}$ & $0.8 \mu \mathrm{m}(-300 \mathrm{MPa})$ & $\Delta \mathrm{L}=16.2 \mu \mathrm{m}$ & -3.69 & +5.13 \\
\hline Wet & $W_{1}=1.85 \mu \mathrm{m} / \mathrm{W}_{2}=2.00 \mu \mathrm{m}$ & $0.8 \mu \mathrm{m}(-300 \mathrm{MPa})$ & $\Delta \mathrm{L}=21.6 \mu \mathrm{m}$ & -2.55 & +3.45 \\
\hline Dry & $W_{1}=1.1 \mu \mathrm{m} / \mathrm{W}_{2}=3.8 \mu \mathrm{m}$ & $0.8 \mu \mathrm{m}(-300 \mathrm{MPa})$ & $\Delta \mathrm{L}=20.1 \mu \mathrm{m}$ & -3.68 & +5.14 \\
\hline Nominal & $W_{1}=2.0 \mu \mathrm{m} / \mathrm{W}_{2}=2.0 \mu \mathrm{m}$ & $0.8 \mu \mathrm{m}(-300 \mathrm{MPa})$ & $\Delta \mathrm{L}=20.3 \mu \mathrm{m}$ & -3.65 & +5.17 \\
\hline Wet & $W_{1}=1.5 \mu \mathrm{m} / \mathrm{W}_{2}=4.0 \mu \mathrm{m}$ & $0.8 \mu \mathrm{m}(-300 \mathrm{MPa})$ & $\Delta \mathrm{L}=23.3 \mu \mathrm{m}$ & -2.47 & +3.53 \\
\hline
\end{tabular}

Table 7.1: A list of design parameters for the zero-order AWGs. The spatial separation $d_{s}$ are the distance measured from the reference line, as shown in Fig. 7.5. The etch depth of all waveguides are $D=1.5 \mu \mathrm{m}$.

\subsection{Photomask Layout}

The commercial software, AutoCad ${ }^{\circledR}$, is used to generate the optical masks of the zeroorder AWG devices. The coordinates of the zero-order AWG waveguide array layout were first calculated using a custom-designed MATLAB program, and then exported to a script file for AutoCad. The details of the simulation code can be found in Appendix B. The test devices include designs with the nominal dimensions and layout, as well as a wide range of variations to accommodate the as-processed feature dimensions, oxide thickness and stress. For example, several oxide patches with a $\pm 30 \%$ variation in length increment $\Delta L$ were added in the optical masks. In addition, one variation included a set of output waveguides whose orientation was shifted by $0.2^{\circ}$ along the output aperture of the FPR region such that the output waveguides were placed at exactly halfway between the original waveguide gaps. Furthermore, the spacing between the input waveguides was deliberately set to be different than that between the output waveguides. The incommensurate spacing of the input and output waveguides should ensure that at least one input will couple to an output waveguide.

Figs. 7.8 shows the final design layout of the test structures. The overall device size 
of an AWG-based splitter is $\sim 12 \mathrm{~mm} \times 4 \mathrm{~mm}$. In each mask, there are two types of layers: ridge layer (labeled as " $\mathrm{R}$ ") and oxide cladding layer (labeled as " $\mathrm{O}$ "). Two design of the "R" and "O" layers are shown, for the devices fabricated with the wet- and dryetching, respectively. All layers are "light field", meaning the digitized data enclosed by the defined boundaries are chrome (for protection), and data outside the boundaries are clear (for exposure). The photomask was written by E-beam lithography, provided by Compugraphics USA Inc. 


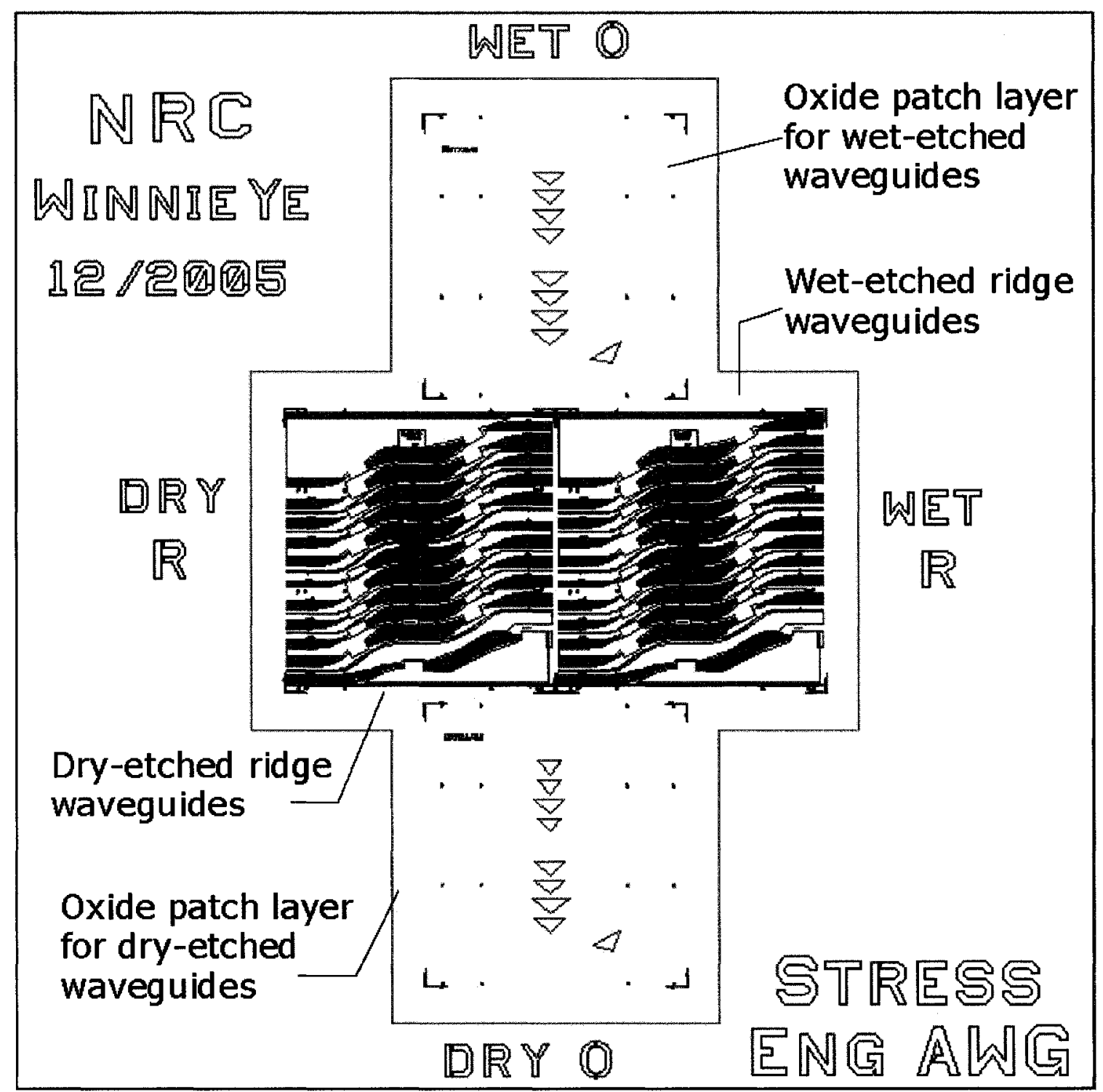

Figure 7.8: Optical masks of zero-order arrayed waveguide grating (AWG) test devices. The overall device size of an AWG-based splitter is $\sim 12 \mathrm{~mm} \times$ $4 \mathrm{~mm}$. 


\subsection{Fabrication}

A fabrication process similar to that described in Chapter 6.6 was used for the AWGbased polarization splitters. There was only one main difference in processing: the AWG waveguides were fabricated using both dry- and wet-etching. Fig. 7.9 compares the drywith the wet-etched AWG ridge waveguides. The wet-etched waveguides have smoother

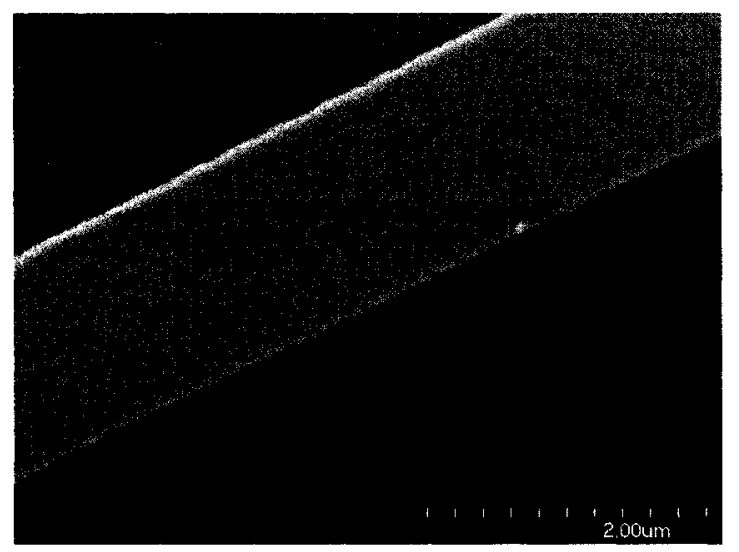

(a)

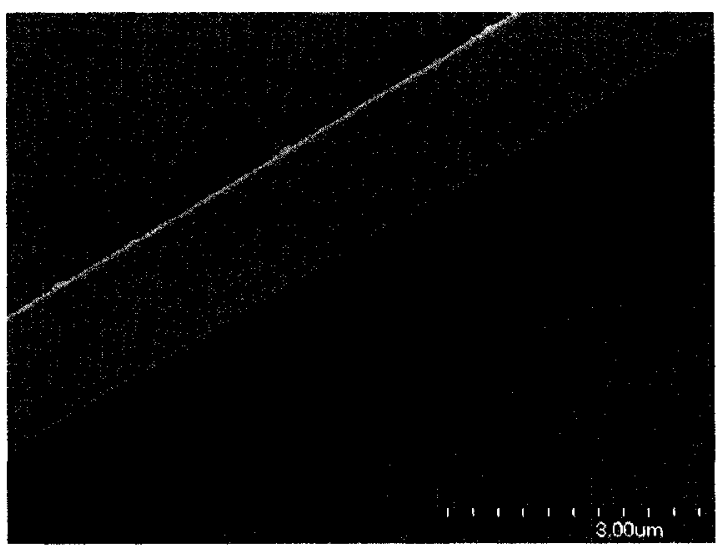

(b)

Figure 7.9: SEM images of (a) a dry-etched ridge waveguide and (b) a wet-etched waveguide.

sidewalls as compared to the dry-etched waveguides, which improve light scattering from the waveguides and reduce the propagation loss. As mentioned earlier in the design and the photomask sections, the layout parameters are specific to each etching process.

For both dry- and wet-etched cases, a simpler wet-etching process was used for patterning the oxide upper cladding because the oxide patches involved in the AWG devices were much larger in size than those in the MZI designs. Fig. 7.10 compares the wet-etched oxide cladding on the dry- and wet-etched ridge waveguides. The oxide cladding has a relatively poor sidewall coverage on the dry-etched ridge waveguides (Fig. 7.10a), similar to the dry-etched MZI device observed in Fig. 6.17. In contrast, the cladding sidewall coverage is much better for the wet-etched waveguides, as illustrated in Fig. 7.10b.

Fig. 7.11 shows the fabricated AWG devices with triangular cladding regions of varied patch sizes. The AWG devices have 100 waveguide channels in the arrayed grating section, 
with 13 inputs and 17 output waveguides. The spacing between the input and output waveguides are different.
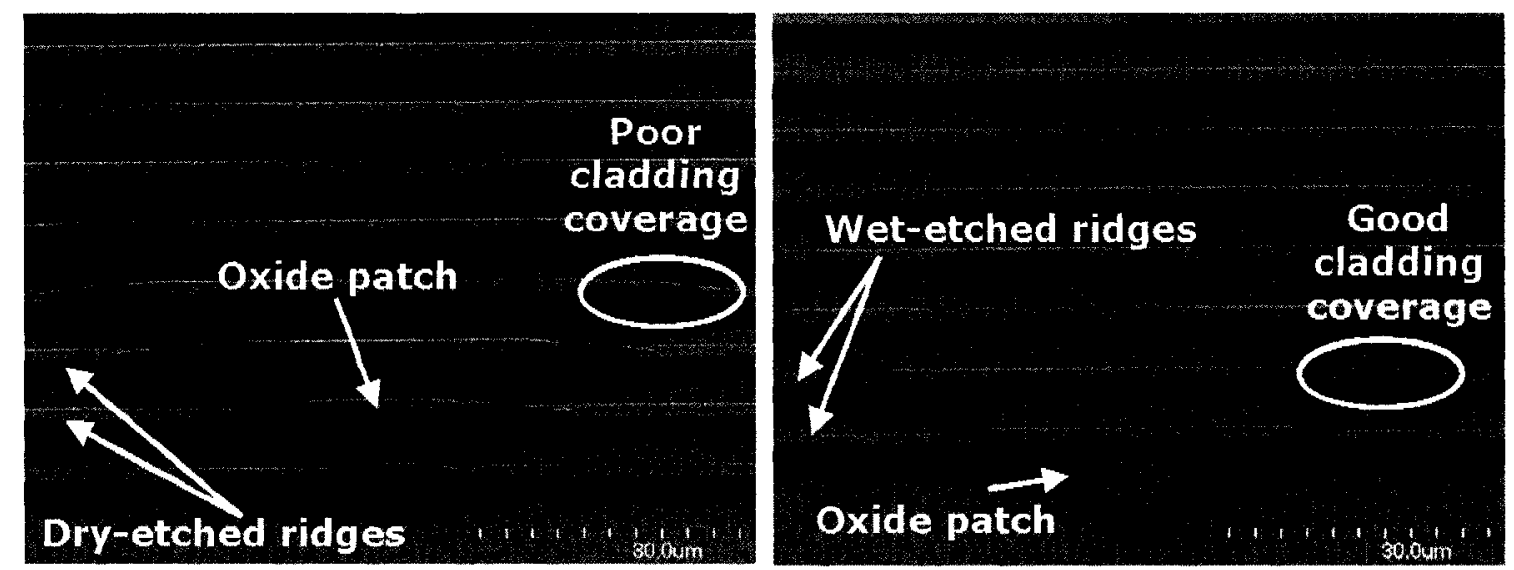

Figure 7.10: SEM images of the wet-etched oxide cladding on (a) the dry-etched ridge waveguides and (b) the wet-etched ridge waveguides.

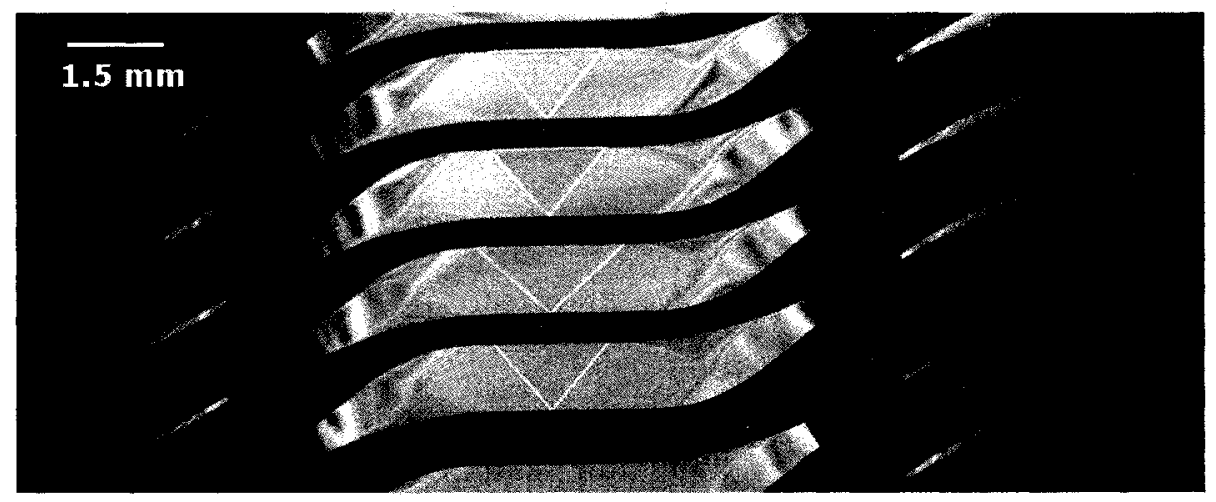

Figure 7.11: A top view of our fabricated wavelength independent zeroorder AWG polarization splitter. There are 100 waveguides in each arrayed section. The triangular windows define the oxide cladding patch regions.

\subsection{Optical Measurements}

The experimental setup used to characterize the AWG-based splitters is the same as the one used for the MZI-based devices in Fig. 6.18. Fig. 7.12 shows the measured wavelength dependence of zero-order AWGs without any oxide cladding. The output power of the TE and TM modes were measured separately, from the same output waveguide. Due to 


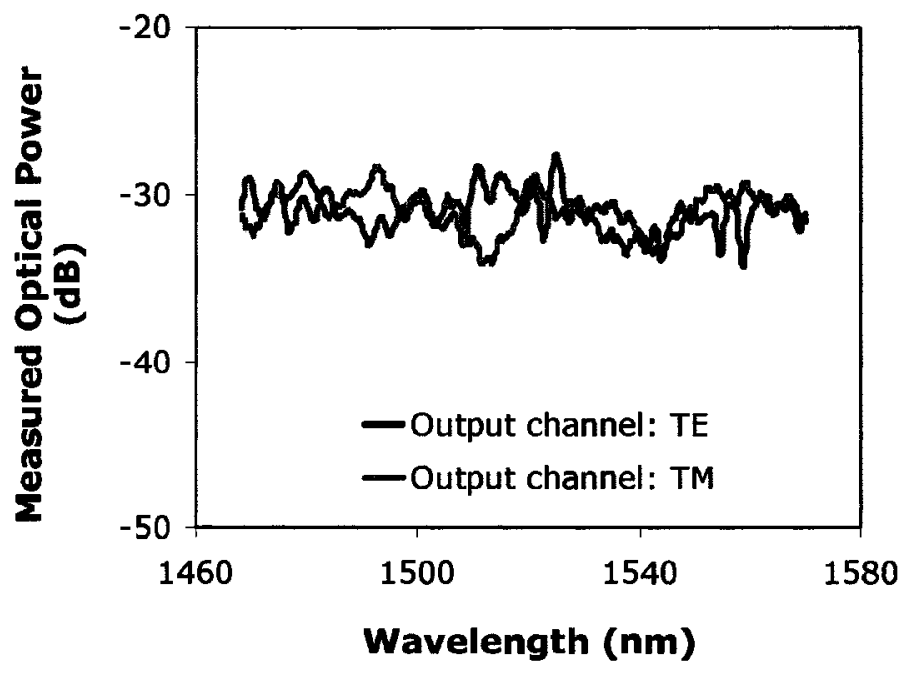

(a)

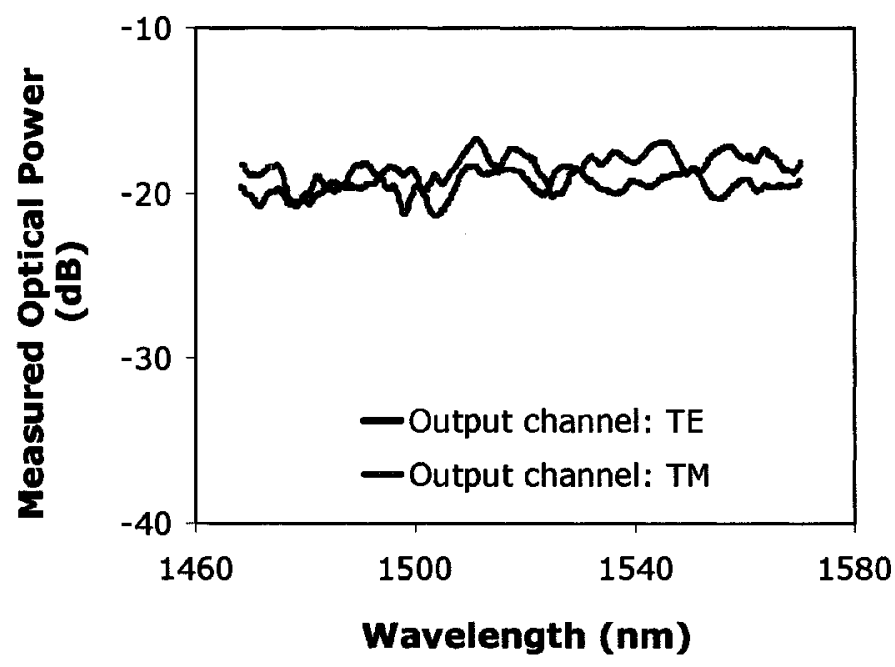

(b)

Figure 7.12: Measured wavelength dependence of a zero-order AWG before oxide cladding deposition: (a) a dry-etched AWG and (b) a wet-etched AWG. The power of both polarizations were measured from the same output channel. All ridge waveguides in the AWGs are designed to have a width $W=2 \mu \mathrm{m}$ and an etch depth $D=1.5 \mu \mathrm{m}$.

the perfect symmetry of the device, the output is almost an exact replica of the input. The identical channel length in the arrayed section ensures the wavelength independent operation. Fig. 7.12(a) and (b) demonstrate this behavior across the entire operating wavelength range of the tunable laser between $1460 \mathrm{~nm}$ and $1570 \mathrm{~nm}$, for a dry- and 
wet-etched AWG device, respectively. The insertion losses of the devices, defined by the ratio of the total output power to the input power, were $-30 \mathrm{~dB}$ and $-20 \mathrm{~dB}$ for the dry- and wet-etched AWG, respectively. The fiber coupling loss on both ends of device, $\mathrm{a} \sim 3 \mathrm{~dB}$ Fresnel reflection loss from the two uncoated facets of the device and the propagation loss contribute to the total insertion loss. A reference measurement of a straight dry-etched test waveguide showed an insertion loss only slightly better than -20 dB. In contrast, the wet-etched AWG devices were observed to have much less insertion loss and demonstrated an improved performance. An insertion loss of $-13 \mathrm{~dB}$ was achieved for straight wet-etched ridge waveguides. The extra loss in the dry-etched AWG devices results from the scattering from the rough ridge sidewalls.

After the deposition of an oxide patch with a thickness of $1 \mu \mathrm{m}$ at a stress level of $\sigma_{\text {film }}=-340 \mathrm{MPa}$, the stress induced phase shifts cause the spatial separation between the TE and TM polarization. Fig. 7.13 shows the screen shots of the outputs of a functioning polarization splitter. For each of the two output waveguide channels, the

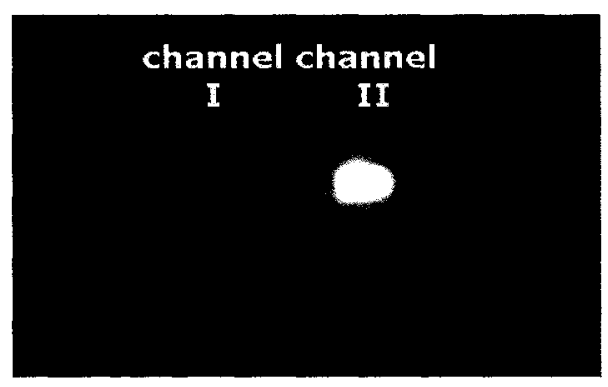

(a)

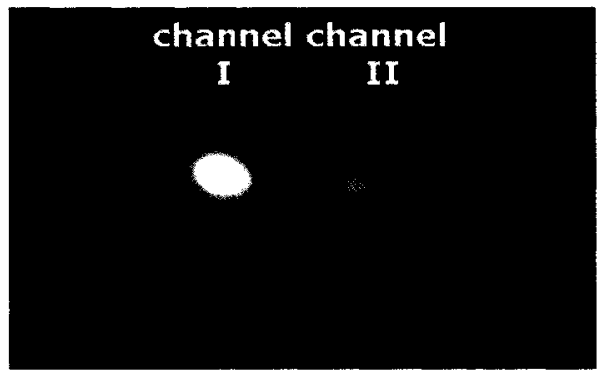

(b)

Figure 7.13: Screen shots of the outputs of a functioning polarization splitter: (a) the TE polarization appears in channel II and (b) the TM polarization appears in channel $I$.

resulting TE and TM power in the waveguide with either a TE or TM polarized input beam were measured. In other words, four measurements were performed for each AWG device: the TE outputs in the channel I and II with an TE input, the TM in output channel I and II with an TM input.

Fig. 7.14 shows the measured data for two functioning polarization splitters, with dry- 
and wet-etched waveguides, respectively. The splitting extinction ratio of the dry-etched AWG polarization splitter was observed to be better than $-10 \mathrm{~dB}$ for both output ports over the entire C-Band, randing from $1510 \mathrm{~nm}$ to $1570 \mathrm{~nm}$, while the wet-etched AWG demonstrated a $-14 \mathrm{~dB}$ extinction ration over a broader wavelength range from 1465 $\mathrm{nm}$ to $1570 \mathrm{~nm}$. The smooth sidewall roughness and the relatively improved cladding coverage on the waveguide sidewalls result in a better device performance in wet-etched AWGs. The highest extinction ratio demonstrated in a wet-etched AWG was $-20 \mathrm{~dB}$, over a $\sim 18 \mathrm{~nm}$ wavelength range, as illustrated in Fig. 7.15.

The measured spatial separations between the TE and TM polarizations $\left(d_{s}^{\mathrm{TE}}\right.$ and $d_{s}^{\mathrm{TM}}$ ) in the devices presented in Figs. 7.14(a), 7.14(b), and 7.15 are listed in Table 7.2. The stress-induced index change $\delta n_{\mathrm{TE}}$ and $\delta n_{\mathrm{TM}}$ can be obtained by re-arranging Eq. 7.5; that is,

$$
\delta n_{s}^{\mathrm{TE}}=\frac{d_{a} n_{s}^{\mathrm{TE}}}{R_{a} L} d_{s}^{\mathrm{TE}} \quad \text { for } \mathrm{TE}, \quad \delta n_{s}^{\mathrm{TM}}=\frac{d_{a} n_{s}^{\mathrm{TM}}}{R_{a} L} d_{s}^{\mathrm{TM}} \quad \text { for } \mathrm{TM}
$$

Overall, there is an excellent agreement between the simulated and the experimentally

\begin{tabular}{|c|c|c|c|}
\hline \multirow{2}{*}{ Parameters } & \multicolumn{3}{|c|}{ Device } \\
\hline & Fig. 17.14a & Fig. 17.14b & Fig. 17.15 \\
\hline 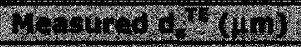 & 2.602 & 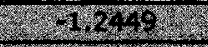 & (. 30026 \\
\hline 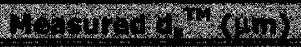 & 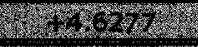 & 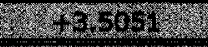 & $40+40955$ \\
\hline (- & 180009 & 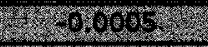 & r. 00.005 \\
\hline 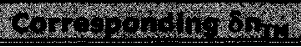 & $25 \times 0,901$ & $8 \times 100018$ & 200.0012 \\
\hline Simulated $\delta n_{\mathrm{TE}}$ & -0.0017 & -0.0010 & -0.0011 \\
\hline Simulated $\delta n_{\mathrm{m}}$ & +0.0024 & +0.0014 & +0.0015 \\
\hline
\end{tabular}

Table 7.2: The measured spatial separations between the TE and TM polarizations in the devices presented in Figs. 7.14(a), 7.14(b), and 7.15.

obtained stress-induced index changes for the TM polarization $\delta n_{\mathrm{TM}}$.

Unlike the MZI-based polarization splitters, almost all of the dry- and wet-etched AWG-based devices on the fabricated sample have demonstrated the polarization splitting function. This implies that the sensitivity to higher-order modes are much less in the AWG-based devices compared to the MZI-based devices. In addition, the AWGs uses 


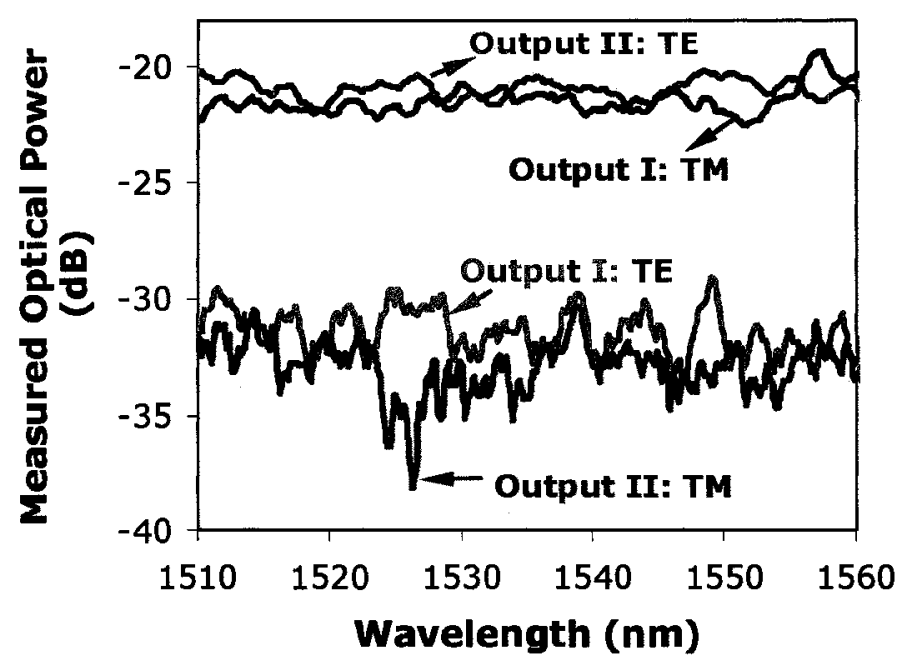

(a)

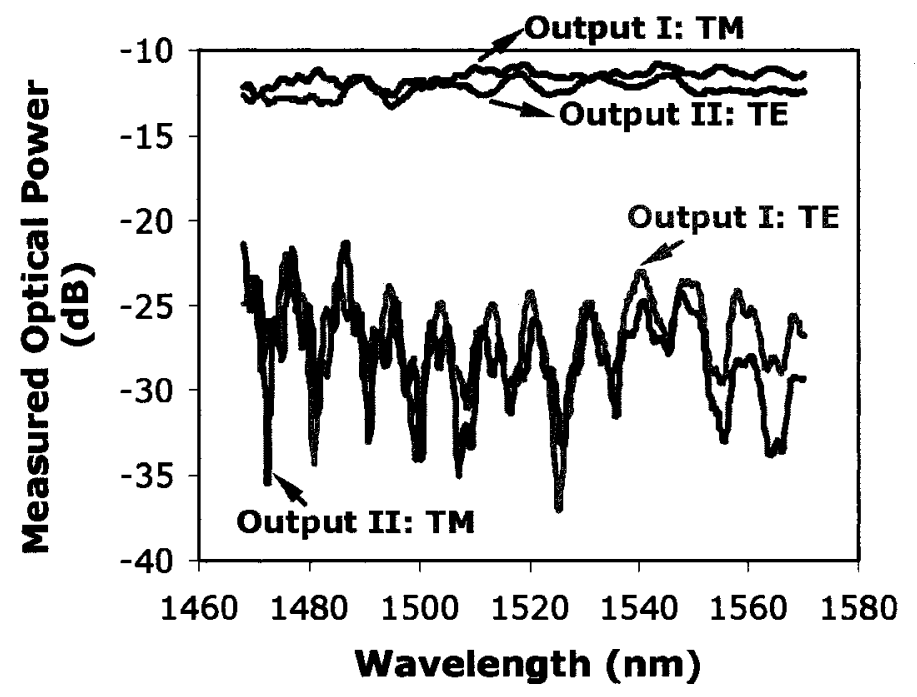

(b)

Figure 7.14: Measured wavelength dependence of zero-order AWG-based polarization splitter with an oxide cladding patch: (a) a dry-etched AWG with an oxide patch length increment $L=20.1 \mu \mathrm{m}$ and (b) a wet-etched AWG with $L=23.3 \mu \mathrm{m}$. The ridge waveguides have a width and etch depth of $2 \mu \mathrm{m}$ and $1.5 \mu \mathrm{m}$, respectively. The oxide cladding has a thickness of $1 \mu \mathrm{m}$ and a stress level of $\sigma_{\text {film }}=-340 \mathrm{MPa}$.

smaller bend radii (with a minimum of $R_{\min }=500 \mu \mathrm{m}$ ) which provide some degrees of mode filtering of the higher-order modes. The AWG devices show significant improvement in the intensity ripples compared with the demonstrated MZI devices in Fig. 6.19. 


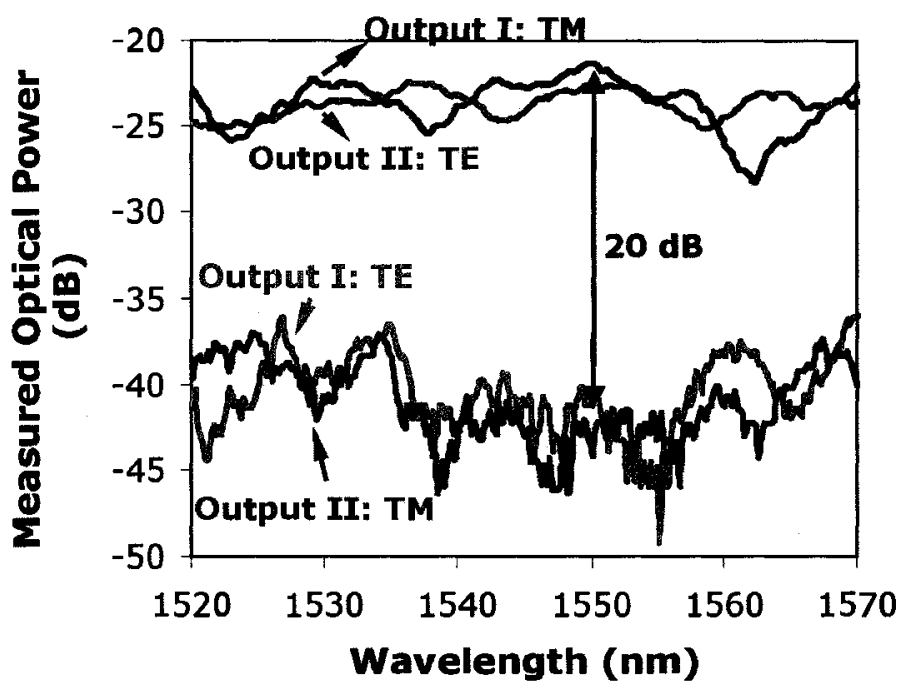

Figure 7.15: A wet-etched zero-order AWG-based polarization splitter with an extinction ratio as high as $-20 \mathrm{~dB}$. The ridge waveguides have a width and etch depth of $W=D=1.5 \mu \mathrm{m}$. The oxide cladding is under a stress level of $\sigma_{\text {film }}=-340 \mathrm{MPa}$, with a thickness of $1 \mu \mathrm{m}$ and a length increment $L=16.4 \mu \mathrm{m}$.

However, the intensity ripple patterns in Figs. 7.14 and 7.15 still suggest phase errors arising in the array section. The dry-etched AWG polarization splitters exhibited a limited $-10 \mathrm{~dB}$ splitting extinction ratio, while the wet-etched AWG splitters demonstrated an extinction ratio as high as $-20 \mathrm{~dB}$.

\subsection{Summary}

The operating principles of novel stress-induced AWG-based polarization splitters were presented in this chapter. The critical design parameters such as the oxide patch length and the spatial separations of the input/output waveguides were investigated. The AWGbased polarization splitters with splitting extinction ratios as high as $-20 \mathrm{~dB}$ were experimentally demonstrated. We conclude that the concept of stress-induced polarization splitting is confirmed, proved, and demonstrated. 


\section{Chapter 8}

\section{Conclusions}

\subsection{Thesis Overview}

The demand for dense integrated optical circuits continues to drive research towards further downscaling device size for more efficient, reliable, economical, and compact integration. High-index-contrast material platforms such as silicon-on-insulator are promising for making highly compact optical devices. However, as the device size reduces, polarization control becomes increasingly challenging. This work has explored and studied the available polarization control methods for optical waveguide devices. The thesis proposes the use of stress engineering for polarization control in the SOI system and presents a calculation scheme for stress analysis in optical waveguides. A detailed investigation of the geometrical and stress-induced effects on waveguide birefringence is presented. From the simulation and experimental results, it is concluded that stress engineering is a powerful and effective tool in birefringence tuning. The second half of the research involves the modeling, design, fabrication, and characterization of two classes of passive polarization splitting and filtering devices which employ the cladding induced stress for polarization tuning. Functioning stress-induced polarization splitters have been successfully demonstrated experimentally, exhibiting broadband polarization splitting with an 
extinction ratio of $-14 \mathrm{~dB}$ for both output polarizations across the entire operating wavelength range of the tunable laser between $1460 \mathrm{~nm}$ and $1570 \mathrm{~nm}$. An extinction ratio as high as $-20 \mathrm{~dB}$ over $18 \mathrm{~nm}$ range has been demonstrated by the wet-etched zero-order AWG based polarization splitters. The outcome of this research project confirms the usefulness, practicality, and effectiveness of the stress engineering approach for waveguide polarization control, and demonstrated working stress-induced polarization splitters.

\subsection{Original Contributions}

The thesis research was carried out at the Optoelectronics group at the National Research Council Canada (NRC). Stress engineering has been independently proposed by our group for polarization management in SOI waveguides [92]. Although the stress method has been widely used for other material systems such as silica, the impact of stress in SOI waveguides has not been carefully investigated. There was no previous published research on stress engineering in SOI systems besides the patent filed by Bookham Technology [68]. In order to model the stress-induced effects in optical waveguides, Chapter 3 demonstrated the development of a new theoretical model, the "Normalized Plane Strain Model", which produced virtually identical stress and birefringence results as a fully generalized model, yet required an order of magnitude less computation time. To the best of our knowledge, this is the first time such a simplified and accurate model has been derived and investigated for stress calculations in optical waveguides. Although the derivation of the model was a collaborative effort with Dr. S. Janz and Dr. D.-X. $\mathrm{Xu}$ from NRC, the original contributions of the project include the implementation of the new numerical calculation scheme and the extensive numerical simulations for the comparisons among the different plane strain models.

The geometrical and stress-induced effects on waveguide polarization using the newly proposed calculation scheme were investigated in Chapter 4. This thesis work presents a 
systematic and comprehensive study of the stress-induced effects, highlighting its promising potential in polarization management for SOI waveguides.

In Chapter 5, the numerical results generated by the implemented simulation models were compared to the experimental measurements obtained by Ms. M.-J. Picard and Dr. D.-X. Xu. The agreement between the simulation results and the experimental data validated the calculation scheme and further confirmed the effectiveness of the stress approach.

Finally, Chapter 6 and 7 presented the modeling, design, and characterization of novel passive polarization devices using stress as a polarization compensator. All aspects of the theoretical development of these devices, including the equation derivations, parameter choices, and optical layout of the test devices were original work. Adtek Photomask Inc. and Compugraphics USA Inc. provided the photomasks of the test devices written by E-beam lithography. These devices were fabricated by Dr. P. Waldron and the oxide cladding films were deposited by Dr. J. Caballero from the Nanofabrication group at the NRC and the Canadian Photonic Fabrication Center (CPFC), respectively. The author has not participated in any part of the fabrication procedure. However, the author performed all the measurements and characterization of the devices. Broadband stress-induced polarization splitters were successfully demonstrated with a high splitting extinction ratio.

\subsection{Significance of Work}

It has been shown that the traditional approach of geometry modification is impractical for birefringence control in SOI devices with small dimensions. Inspired from the extensive research in elimination of stress in glass planar waveguide devices, this work proposed the use of stress engineering for polarization control in a high-index contrast system such as SOI waveguides. Our experiments have demonstrated that the stress 
engineering approach is effective for waveguide devices with small dimensions. The main advantage of this approach is its simplicity - only one single post-fabrication deposition step is required for polarization control. In addition, this method offers the freedom to decouple the birefringence constraints from the waveguide design because stress birefringence can be induced for waveguides of arbitrary shape. Therefore the waveguide can be designed to independently optimize its insertion loss, coupler performance and bend loss. Furthermore, the devices have the advantage of broadband performance because the stress-induced effects are relatively independent of wavelength. Finally, the novel passive stress-induced SOI polarization splitters and filters, designed and demonstrated in Chapter 6 and 7, are useful in defining the near-term commercial applications.

The significance of this research work is evidenced by the accepted publications and presentations in scientific and engineering journals and conferences as listed below:

\section{Refereed Journal Papers}

1. W. N. Ye, D.-X. Xu, S. Janz, P. Cheben, M.-J. Picard, B. Lamontagne, and N. G. Tarr, "Birefringence Control Using Stress Engineering for Silicon-on-insulator (SOI) Waveguides," IEEE J. Lightw. Technol., vol. 23, no. 3, pp. 1308-1318, Mar. 2005 .

2. D.-X. Xu, P. Cheben, D. Dalacu, A. Delage, S. Janz, B. Lamontagne, M.-J. Picard, W. N. Ye, "Eliminating the birefringence in silicon-on-insulator ridge waveguides using the cladding stress," Opt. Lett., vol. 29, no. 20, pp. 2384-2386, Oct. 2004.

\section{Book Chapters}

1. S. Janz, P. Cheben, A. Delage, B. Lamontagne, M.-J. Picard, D.-X. Xu, K.P. Yap, W. N. Ye, "Microphotonics: Current Challenges and Applications," in Frontiers in Planar Lightwave Circuit Technology, S. Janz,J. Ctyroky, S. Tanev eds., pp. 1-38, Springer: Dordrecht, 2006. 


\section{Refereed Conference Papers}

1. D.-X. Xu, P. Cheben, A. Delge, S. Janz, B. Lamontagne, M.-J. Picard, E. Post, P. Waldron and W. N. Ye, "Applications of Cladding Stress Induced Effects For Advanced Polarization Control in Silicon Photonics," accepted for an oral presentation at the Progress In Electromagnetics Research Symposium (PIERS'07), Beijing, China, Mar. 2007 (invited).

2. W. N. Ye, D.-X. Xu, S. Janz, P. Waldron, and N. G. Tarr, "Wavelength-independent Stress-induced SOI Polarization Splitters," accepted for an oral presentation at the Photonics West 2007, San Jose, USA, Jan. 2007.

3. D.-X. Xu, S. Janz, P. Waldron, W. N. Ye, "Polarization-Insensitive Ring Resonators in SOI Using Cladding Stress," accepted for an oral presentation at the Photonics West 2007, San Jose, USA, Jan. 2007 (invited).

4. S. Janz, P. Cheben, A. Delage, A. Densmore, B. Lamontagne, E. Post, J. Schmid, P. Waldron, D.-X. Xu, K.P. Yap, and W. N. Ye, "Silicon Microphotonic Waveguide Technology for Sensing, Spectroscopy, and Communications," the Electrochemical Society Fall Meeting (ECS06), Cancun, Mexico, Oct. 2006, vol. 3(11), pp. 61-78 (invited).

5. W. N. Ye, D.-X. Xu, S. Janz, P. Waldron, and N. G. Tarr, "Stress-induced SOI Polarization Splitter Based on Mach-Zehnder Interferometers (MZI)," LEOS $3^{\text {rd }}$ International Conference on Group IV Photonics, Ottawa, Canada, Sept. 2006.

6. D.-X. Xu, W. N. Ye, P. Cheben, A. Delage, S. Janz, B. Lamontagne, M.-J. Picard, E. Post, "FEM Simulation Assisted Stress-Engineering for PolarizationControl in SOI Waveguide Components," Integrated Photonics Research Topical Meeting (IPR06), Connecticut, USA, Apr. 2006 (invited).

7. D.-X. Xu, S. Janz, P. Cheben, W. N. Ye, "Design of Polarization-Insensitive SOI Ring Resonators Using Cladding Stress-Induced Birefringence and MMI Coupler", LEOS $2^{\text {nd }}$ International Conference on Group IV Photonics, Antwerp, Belgium, Sept. 2005 (invited).

8. W. N. Ye, D.-X. Xu, S. Janz, P. Cheben, A. Delage, N. G. Tarr, "Novel Stressinduced Passive Polarization Splitters/Filters in Silicon-on-Insulator", the $12^{\text {th }}$ Cana- 
dian Semiconductor Technology Conference, Ottawa, Ontario, Aug. 2005.

9. D.-X. Xu, A. Delge, P. Cheben, B. Lamontagne, S. Janz, W. N. Ye, "Silicon-oninsulator (SOI) as a photonics platform", $12^{\text {th }}$ International Symposium on Siliconon-Insulator Technology and Devices, Quebec City, May 2005 (invited).

10. S. Janz, P. Cheben, A. Delage, B. Lamontagne, M.-J. Picard, D.-X. Xu, K.-P. Yap and W. N. Ye, "Enabling technologies for silicon-based microphotonics," Integrated Photonics Research and Applications/Nanophotonics for Information Systems Topical Meetings (IPRA'05), San Diego, CA, Feb. 2005.

11. D.-X. Xu, W. N. Ye, A. Bogdanov, D. Dalacu, A. Delge, P. Cheben, S. Janz, B. Lamontagne, M.-J. Picard, N. G. Tarr, "Design of polarization-insensitive components using geometrical and stress-induced birefringence in SOI waveguides," in Proc. SPIE Int. Soc. Opt. Eng., vol. 5730, Jan. 2005 (invited).

12. D.-X. Xu, J.-M. Baribeau, P. Cheben, D. Dalacu, A. Delage, B. Lamontagne, S. Janz, M.-J. Picard, W. N. Ye, "Prospects and challenges for microphotonic waveguide components based on Si and SiGe," Electrochemical Society Fall Meeting (ECS'04)-SiGe: Materials Processing and Devices, Honolulu, Hawaii, vol. 2004-07, Oct. 2004, pp. 619-633.

13. S. Janz, P. Cheben, A. Delage, B. Lamontagne, M.-J. Picard, D.-X. Xu, K.-P. Yap and W. N. Ye, "Silicon-Based Integrated Optics: Waveguide Technology to Microphotonics," Materials Research Society Fall Meeting (MRS'04) - Group IV Semiconductor Nanostructures, Boston, MA, Nov. 2004.

14. S. Janz, P. Cheben, A. Delage, B. Lamontagne, M.-J. Picard, D.-X. Xu, K. P. Yap, and W. N. Ye, "Microphotonics: current challenges and applications," NATO Advanced Research Workshop on Frontiers in Planar Lightwave Circuit Technology, Ottawa, Canada, Sept. 2004 (invited).

15. S. Janz, P. Cheben, D. Dalacu, A. Delage, B. Lamontagne, M.-J. Picard, D.-X. $\mathrm{Xu}, \mathrm{W} . \mathrm{N}$. Ye, "Tuning the modal birefringence in waveguide devices," Integrated Photonics Research Topical Meeting (IPR'04), San Francisco, California, USA, Jun. 2004. 
16. W. N. Ye, D.-X. Xu, S. Janz, P. Cheben, M.-J. Picard, B. Lamontagne, and N. G. Tarr, "Stress Induced Birefringence in Silicon-on-insulator (SOI) Waveguides," in Proc. SPIE Int. Soc. Opt. Eng., vol. 5357, pp. 57-66, Jan. 2004.

17. D.-X. Xu, S. Janz, P. Cheben, M.-J. Picard, B. Lamontagne, and N. G. Tarr, W. N. Ye, "Control and compensation of birefringence in SOI waveguides," Technical Digest, IEEE Lasers and Electro-Optics Society: LEOS 16th Annual General Meeting, Tucson, Arizona, USA, pp. 590-591, Sep. 2003.

\section{Non-refereed Conference Presentations}

1. W. N. Ye, D.-X. Xu, S. Janz, P. Waldron, N. Garry Tarr, "Stress Engineering For Polarization Control In SOI And Its Applications In Novel Passive Polarization Splitters/Filters," poster presentation at the Canadian Institute for Photonic Innovations (CIPI) Student Conference 2006, Quebec City, Canada, June 2006.

2. W. N. Ye, D.-X. Xu, S. Janz, P. Cheben, B. Lamontagne, N. Garry Tarr, "Modeling, Design, and Fabrication of Novel Photonic Devices," poster presentation at the Japan Society for the Promotion of Science (JSPS) Summer Exchange Program, Sokendai, Hayama, Japan, June 2005.

3. W. N. Ye, D.-X. Xu, S. Janz, P. Cheben, B. Lamontagne, N. Garry Tarr, "Siliconon-Insulator (SOI) Platform For Making Multifunctional and High-density Integrated Photonics Devices," oral presentation at the German-Canadian Young Scientists Photonics Forum, Munich, Germany, June 2005.

4. W. N. Ye, D.-X. Xu, S. Janz, P. Cheben, B. Lamontagne, M.-J. Picard, N. Garry Tarr, "Stress Engineering in Silicon-on-insulator (SOI) waveguides interferometric devices," poster presentation at the Workshop for Si-based Photonics, Hamilton, ON, Canada, Nov. 2004.

5. W. N. Ye, D.-X. Xu, S. Janz, P. Cheben, B. Lamontagne, M.-J. Picard, N. Garry Tarr, "Stress Engineering for birefringence control in Silicon-on-insulator (SOI) waveguides," poster presentation, Photonics Research Ontario (PRO) Student Forum, Ottawa, ON, Canada, Sep. 2004 (invited).

6. W. N. Ye, D.-X. Xu, S. Janz, P. Cheben, B. Lamontagne, M.-J. Picard, N. Garry Tarr, "Birefringence Control Using Stress Engineering for Silicon-on-insulator (SOI) 
Waveguides," oral presentation, Canadian Institute for Photonic Innovations (CIPI) Student Conference 2004, Sherbrooke, QC, Canada, Jun. 2004.

\subsection{Final Remarks}

This work has explored a new polarization management method by using cladding induced stress. The usefulness, practicality, and effectiveness of the approach were confirmed by experimental results. Novel broadband stress-induced polarization splitters on SOI platform were proposed, designed, fabricated, and characterized. These devices provide the fundamental operations in optical signal processing and monitoring, with applications ranging from network monitoring, polarization mode dispersion compensation, to polarimetric sensors.

Many challenges still remain in the development of SOI-based microphotonics into a commercially viable technology. The fiber to waveguide coupling, propagation loss due to scattering, and polarization dependence are some of the key issues [15]. This research project focuses to address the polarization dependence related problems by utilizing cladding induced stress and by implementing the stress-induced polarization splitters. The existing choices of the waveguide structure and the cladding material offer enormous opportunities for improvement and optimization of the future device generations in terms of smaller device footprints, enhanced functionality and performance, and increased tolerances to fabrication inaccuracies. In addition, further research for efficient coupling and improved fabrication techniques are necessary. Finally, the potential of the tunability of the stress compensation method should be explored. 


\section{Appendix A}

\section{Stress And Modal Analysis Using}

\section{FEMLAB ${ }^{\circledR}$}

The commercial software package, FEMLAB $^{\circledR}$, has been used for the stress and modal analysis for most of the simulations presented in this thesis. The following section includes a sample programming code for calculating the stress-induced effective index of a ridge waveguide.

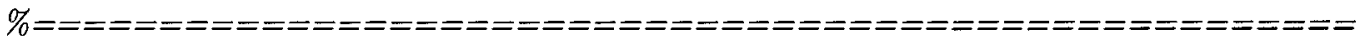

\% FEMLAB simulation code for the stress and model analysis

$\%$ of a ridge waveguide system

$\%$ Written by: Winnie Ye

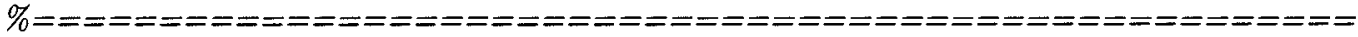

\% This is a sample code for calculating the effective indices of the

$\%$ first four waveguide modes of a ridge waveguide under stress.

clear all;

flclear fem;

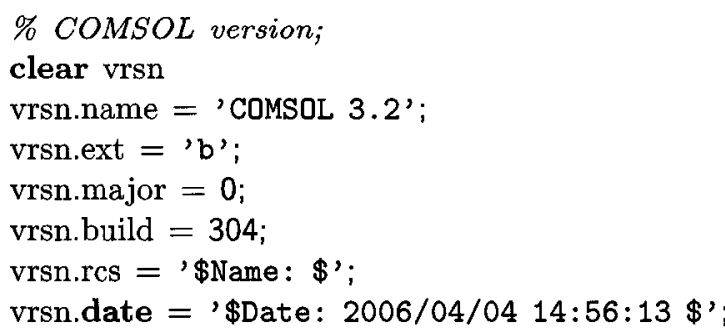


fem.version $=$ vrsn;

$\%$ Define the basic geometrical parameters of the ridge waveguides

$\mathrm{w} 1=1.5 \mathrm{e}-6 ; \%$ Top ridge width

w2=1.5e-6; \% Bottom ridge width

$10=1.55 \mathrm{e}-6 ; \%$ Operating wavelength

$\mathrm{k}=0.2 \mathrm{e}-6 ; \%$ Upper oxide cladding thickness

$\mathrm{d}=1.5 \mathrm{e}-6 ; \%$ Ridge etch depth

$\mathrm{h}=2.2 \mathrm{e}-6 ; \%$ Ridge height

angle $=54 ; \%$ Ridge sidewall angle in degrees

mode_AWG $=[] ; \%$ fem solution matrix

\% This example scans the etch depth $D$ from 1 um to $2 u m$

for $d=1 e-6: 0.1 e-6: 2 e-6$

$\mathrm{z} 1=\mathrm{w} 1 / 2$;

$\mathrm{z} 2=\mathrm{w} 2 / 2$;

theta=angle*pi/180; \% Ridge sidewall angle in radians

if (theta $\left.{ }^{\sim}=0\right)$

$\mathrm{z} 2=\mathbf{d} / \tan ($ theta $)+\mathrm{z} 1 ; \%$ Calculate the ridge bottom width

$\%$ Calculate the oxide cladding that covers the sidewalls $-70 \%{ }^{*} k$ $\mathrm{ss}=0.7 * \mathrm{k} / \sin ($ theta $)-\mathrm{k} / \tan$ (theta);

else

$\mathrm{ss}=0.7^{*} \mathrm{k}$

end

$\%$ Draw the geometry of the waveguide system

clear draw

\% substrate: 100umX100um

$\mathrm{g} 1=\mathrm{rect} 2(10.0 \mathrm{E}-5,10 \mathrm{E}-5$,' base ', ' corner', 'pos ', $[-5.0 \mathrm{E}-5,-10 \mathrm{e}-5])$;

$\%$ buried oxide cladding: 0.37umX100um

g2 $=\operatorname{rect} 2(10.0 e-5,3.7 \mathrm{E}-7$,' base', ' corner' , 'pos ', $[-5.0 \mathrm{E}-5,0])$;

$\%$ ridge waveguide

carr $=\{$ curve $2([-5.0 e-5,-5.0 e-5],[3.7 E-7, .37 e-6+h-d],[1,1]), \ldots$

curve $2([-5.0 e-5,-z 2],[.37 e-6+h-d, .37 e-6+h-d],[1,1]), \ldots$

curve2 $([-\mathrm{z} 2,-\mathrm{z} 1],[.37 \mathrm{e}-6+\mathrm{h}-\mathrm{d}, .37 \mathrm{e}-6+\mathrm{h}],[1,1]), \ldots$

60

curve $2([-z 1, z 1],[.37 e-6+h, .37 e-6+h],[1,1]), \ldots$

curve2 $([\mathrm{z} 1, \mathrm{z} 2],[.37 \mathrm{e}-6+\mathrm{h}, .37 \mathrm{e}-6+\mathrm{h}-\mathrm{d}],[1,1]), \ldots$

curve $2([z 2,5.0 e-5],[.37 e-6+h-d, .37 e-6+h-d],[1,1]), \ldots$

curve2([5.0e-5,5.0e-5],[.37e-6+h-d,3.7E-7],[1,1]), ..

curve2 $([5.0 e-5,-5.0 e-5],[3.7 \mathrm{E}-7,3.7 \mathrm{E}-7],[1,1])\}$;

g3=geomcoerce('solid', carr);

$\%$ oxide upper cladding region

carr $=\{$ curve $2([-5.0 e-5,-5.0 e-5],[.37 e-6+h-d, .37 e-6+h-d+k],[1,1]), \ldots$

curve $2([-5.0 \mathrm{e}-5,-\mathrm{z} 2-\mathrm{ss}],[.37 \mathrm{e}-6+\mathrm{h}-\mathrm{d}+\mathrm{k}, .37 \mathrm{e}-6+\mathrm{h}-\mathrm{d}+\mathrm{k}],[1,1]), \ldots$

curve $2([-\mathrm{z} 2-\mathrm{ss},-\mathrm{z} 1-\mathrm{ss}],[.37 \mathrm{e}-6+\mathrm{h}-\mathrm{d}+\mathrm{k}, .37 \mathrm{e}-6+\mathrm{h}+\mathrm{k}],[1,1]), \ldots$

curve2 $([-\mathrm{z} 1-\mathrm{ss}, \mathrm{z} 1+\mathrm{ss}],[.37 \mathrm{e}-6+\mathrm{h}+\mathrm{k}, .37 \mathrm{e}-6+\mathrm{h}+\mathrm{k}],[1,1]), \ldots$

curve2 $([\mathrm{z} 1+\mathrm{ss}, \mathrm{z} 2+\mathrm{ss}],[.37 \mathrm{e}-6+\mathrm{h}+\mathrm{k}, .37 \mathrm{e}-6+\mathrm{h}-\mathrm{d}+\mathrm{k}],[1,1]), \ldots$

curve2([z2+ss, 5.0e-5],[.37e-6+h-d+k,.37e-6+h-d+k],[1,1]), .. 


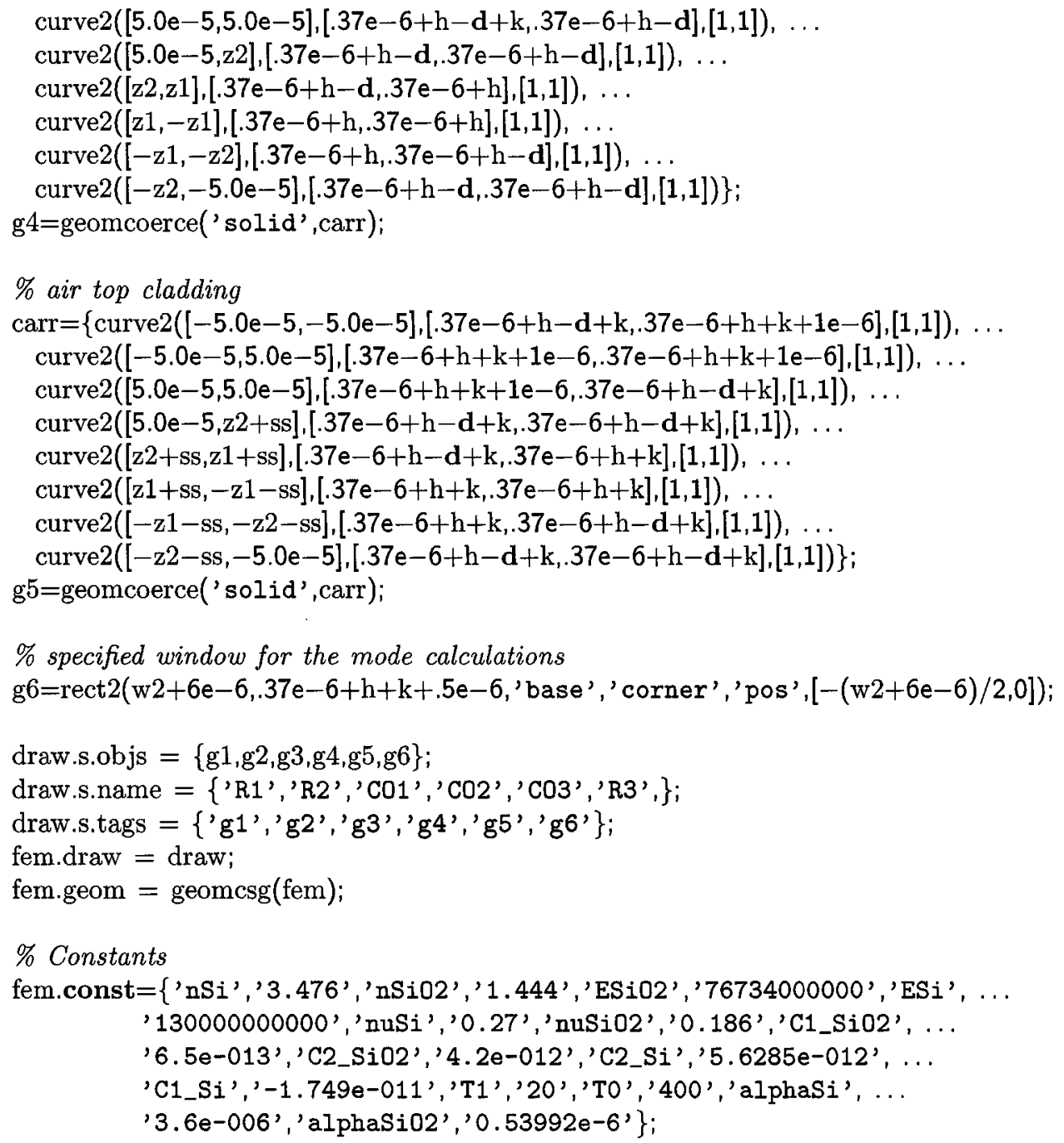

$\%$ Initialize mesh: the corner points of the ridge and the ridge region

$\%$ (Default values are not included)

\% Application mode 1: Define the Plane Stain Model for stress calculation clear appl appl.mode.class = 'SmePlaneStrain'; appl.module $=$ 'SME'; appl.gporder $=4$; appl.cporder $=2$; appl.assignsuffix $=$ '_smpn'; clear pnt pnt. Hy $=\{0,1,1\}$ pnt. $\mathrm{Hx}=\{0,1,0\}$; 


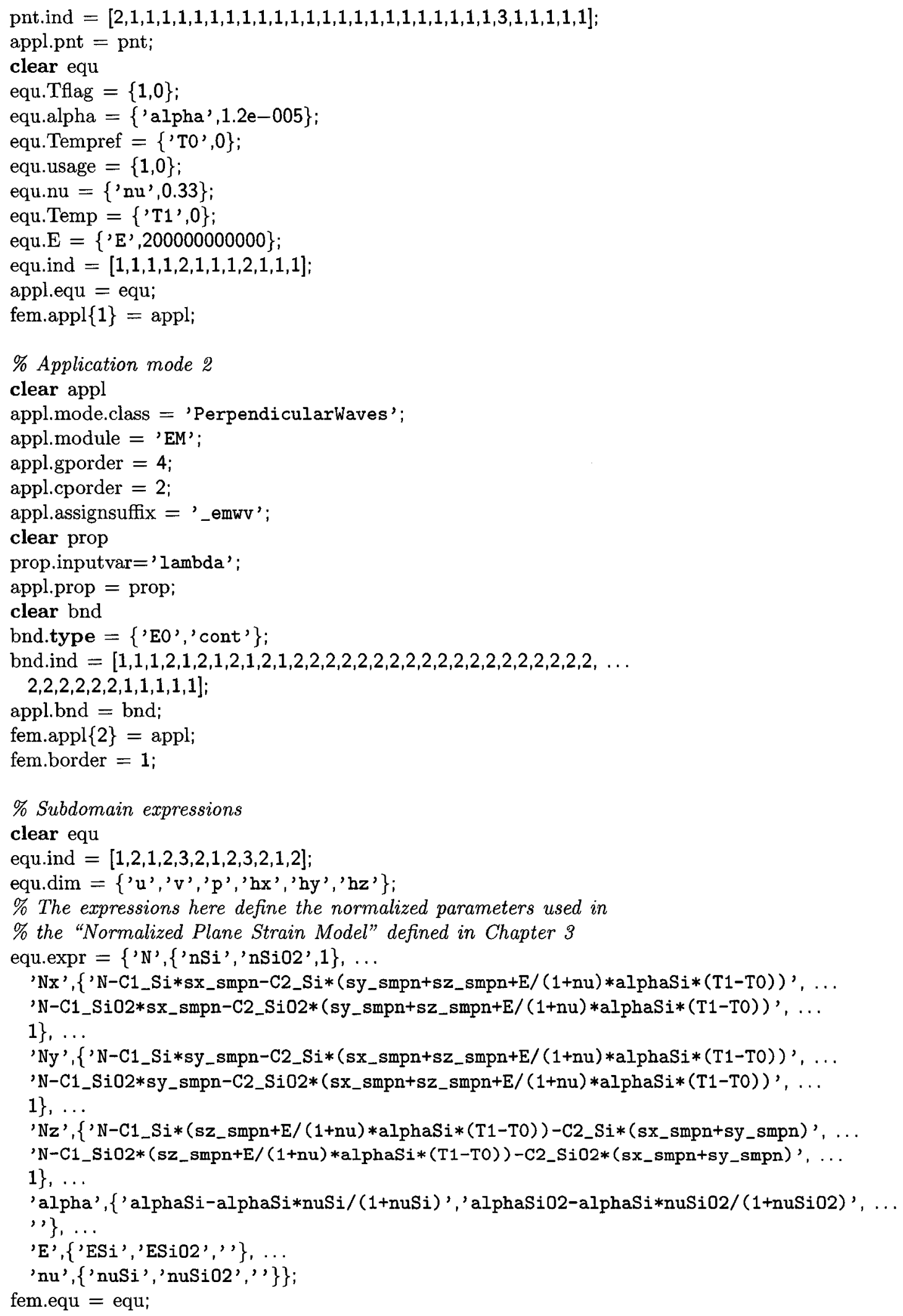




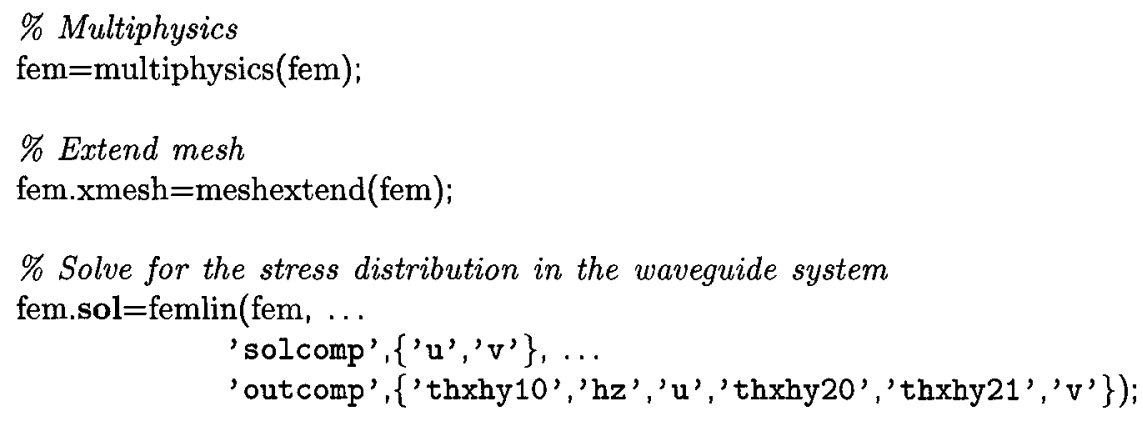

$\%$ Save current fem structure for restart purposes fem $0=$ fem;

$\%$ Plot the stress \sigma_x

figure; postplot(fem, ...

'tridata', \{'sx_pn',' cont', internal'\}, ...

'trimap',' jet(1024)', ...

'title','Surface: sx normal stress global sys.', ...

'refine', $3, \ldots$

'axis', $[-8 \mathrm{E}-6,10 \mathrm{E}-5,-3 \mathrm{E}-6,8 \mathrm{E}-6,-1,1])$;

$\%$ (Default values are not included)

\% Application mode 1: the same Plane Stain Model used for stress calculation clear appl

appl.mode.class = 'SmePlaneStrain';

appl.module = 'SME';

appl.gporder $=4$;

appl.cporder $=2$;

appl.assignsuffix = '_smpn';

clear pnt

pnt. $\mathrm{Hy}=\{0,1,1\}$;

pnt. $\mathrm{Hx}=\{0,1,0\}$;

pnt.ind $=[2,1,1,1,1,1,1,1,1,1,1,1,1,1,1,1,1,1,1,1,1,1,1,1,3,1,1,1,1,1]$;

appl.pnt $=$ pnt;

clear equ

equ. Tflag $=\{1,0\}$;

equ.alpha $=\{$ 'alpha',1.2e-005 $\}$;

equ.Tempref $=\left\{{ }^{\prime} \mathrm{TO}, 0\right\}$;

equ.usage $=\{1,0\}$;

equ.nu $=\{$ 'nu',0.33\};

equ.Temp $=\left\{{ }^{\prime} \mathrm{T} 1\right.$ ',0 0 ;

equ.E $=\left\{{ }^{\prime}{ }^{\prime}, 200000000000\right\} ;$

equ.ind $=[1,1,1,1,2,1,1,1,2,1,1,1]$;

appl.equ = equ;

fem.appl $\{1\}=$ appl;

\% Application mode 2: Model for find the propagation constants of the modes clear appl

appl.mode.class = 'PerpendicularWaves';

appl.module = 'EM'; 


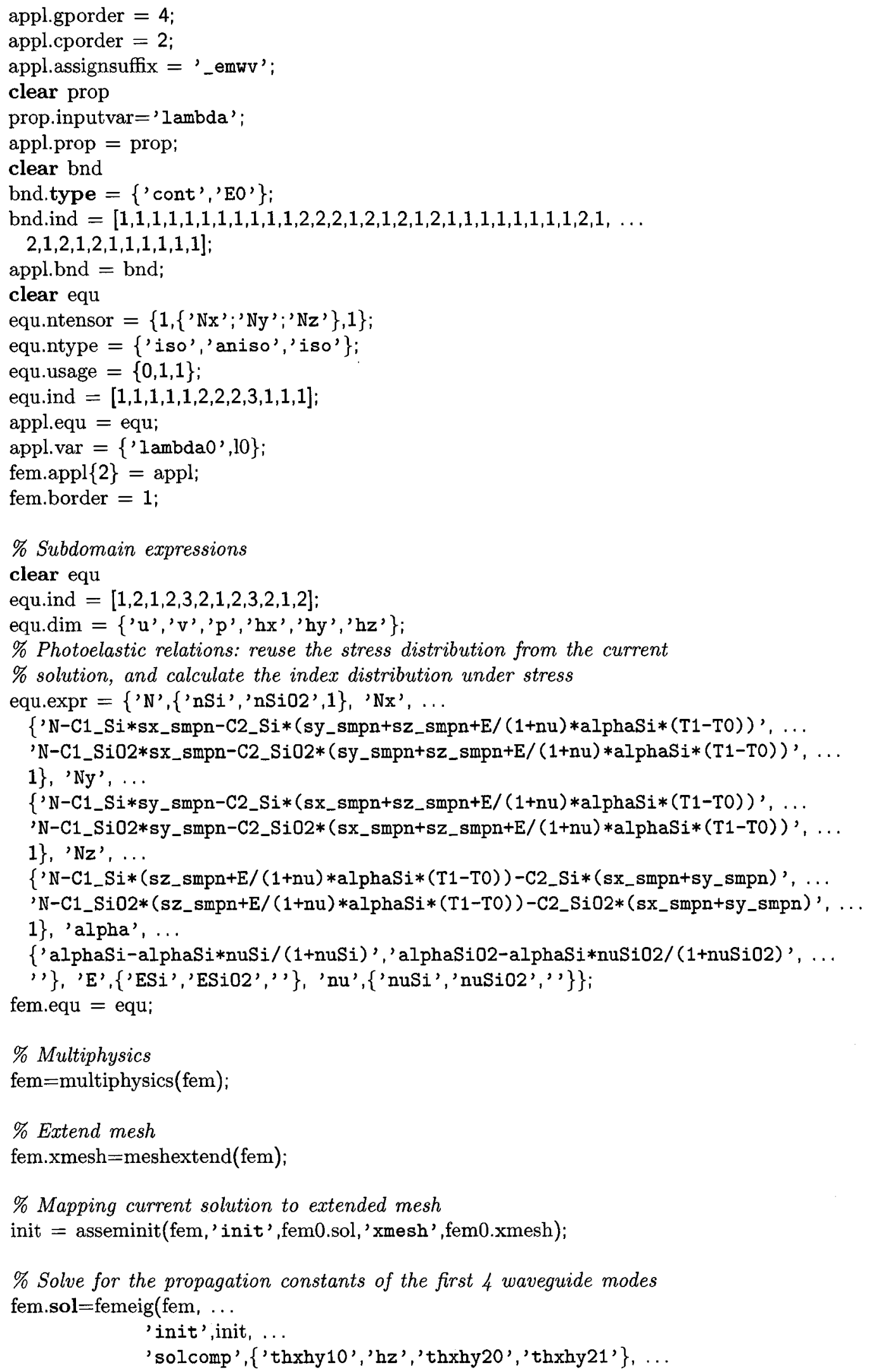




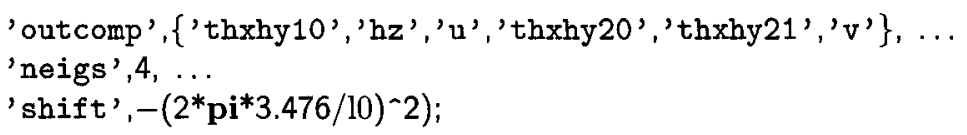

$\%$ Output the effective index of the mode n_index $=\mathbf{s q r t}(-$ fem.sol.lambda $) / 2 / \mathbf{p i}^{*} 10$ $\%$ Saves the fem structure into a matrix mode_AWG $=[$ mode_AWG fem]; end 


\section{Appendix B}

\section{Zero-order AWG Layout Generation}

$\mathrm{C}$ and Visual Basic ${ }^{\circledR}$ are used as the programming language to generate a zero-order arrayed waveguide grating device which has equal spacing between adjacent waveguides at the center of the arrayed section. Fig. B.1 shows the algorithm that is used for the layout generation. A custom-designed automated program calculates the bending radius $(R)$ of every curved waveguide in the arrayed section, and outputs the required lengths of the straight waveguides $\left(l_{0}\right.$ and $\left.l_{1}\right)$ that connects tangentially to the curved ones. The minimum bending radius of SOI waveguides is set to $500 \mu \mathrm{m}$. Given the constant spacing between adjacent waveguides at the middle of the arrayed section (i.e., ConstSep), the length of the $i^{\text {th }}$ horizontal straight waveguide is:

$$
l_{0}=l_{1}+\Delta l=\frac{\text { CenterPA_X }+(\mathrm{i}-1) * \text { ConstSep }-\operatorname{InTipPos} \mathrm{X}}{\sin \theta},
$$

if the coordinates where the input enters the splitter FPR region and the center of the bottom waveguide in the arrayed section are fixed at [InTipPosZ, InTipPosX] and [CenerPA_Z, CenterPA_X], respectively. To draw a curved waveguide which is tangential to $l_{1}$ and the horizontal straight waveguide, we need to define the center of curvature $\left[Z_{r}\right.$, $X_{r}$ ], where

$$
\begin{array}{cc}
X_{r}= & -\tan \alpha\left(Z_{r}-\operatorname{InTipPosZ}-l_{0}\right)+\operatorname{In} \operatorname{TipPosX}, \\
Z_{r}= & -\frac{X_{r}-\operatorname{InTipPosX}}{\tan \alpha}+\operatorname{In} \operatorname{TipPosZ}+l_{0} .
\end{array}
$$




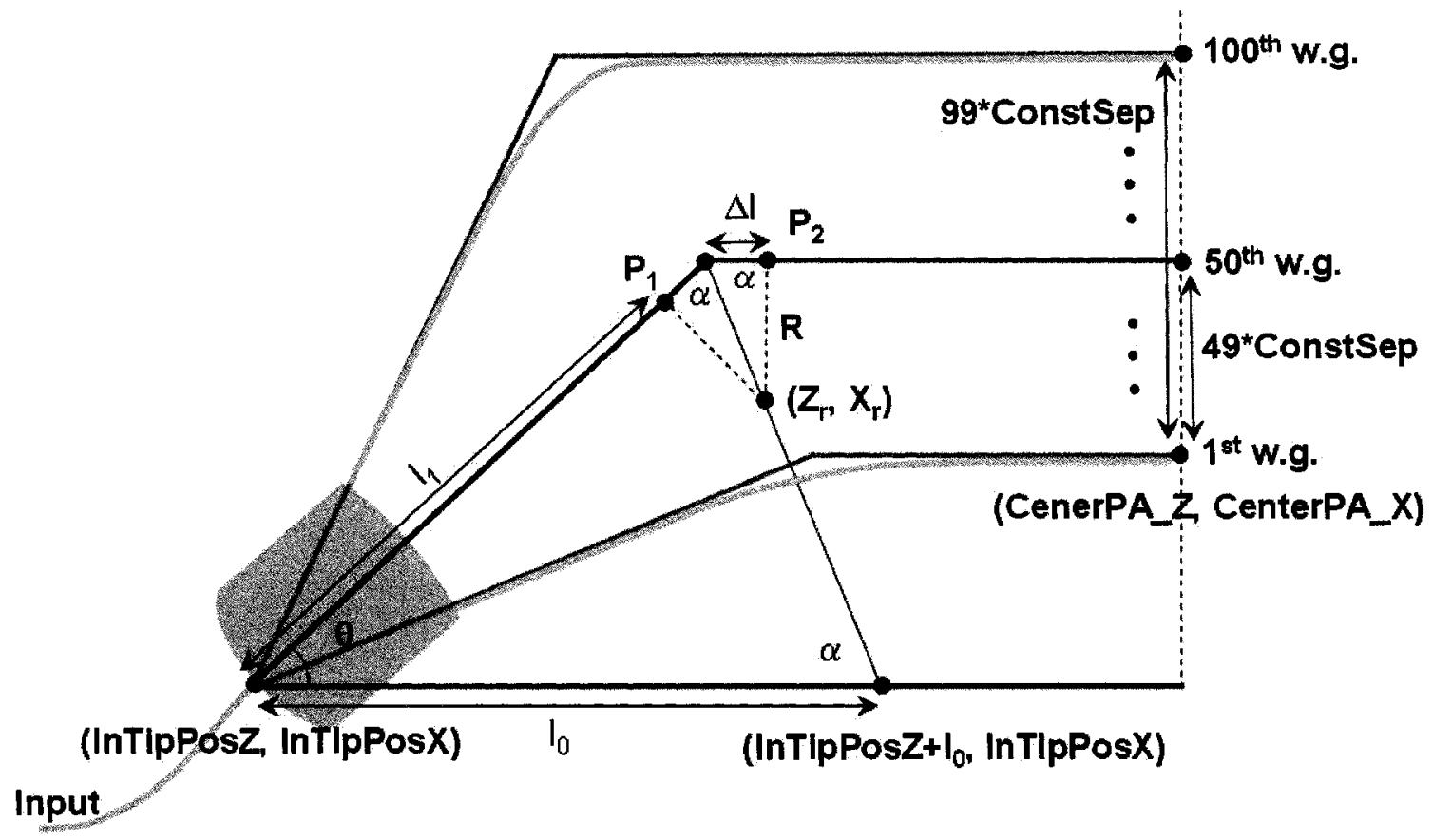

Figure B.1: Algorithm for drawing the layout of a zero-order AWG device (left half) with equal spacing at the center of the arrayed section. The constant spacing between adjacent waveguides at the middle of the arrayed section is "ConstSep". Here $\alpha=90^{\circ}-\frac{\theta}{2}$.

The $\mathrm{P}_{2}$ coordinate for the $i^{\text {th }}$ waveguide is: $\left[Z_{r}\right.$, CenterPA_X $+(\mathrm{i}-1) *$ ConstSep $]$. Since the curved waveguide must be tangentially connected to the two straight waveguides, the coordinate of the $\mathrm{P}_{1}$ point for the $i^{\text {th }}$ waveguide can be obtained as follows:

$$
\begin{array}{r}
\Delta l=\frac{\text { CenterPA_X }+(i-1) * \text { ConstSep }-X_{r}}{\tan \alpha}, \text { and } \\
P_{1}:\left[\left(l_{0}-\Delta l\right) * \cos \theta+\operatorname{InTipPosZ},\left(l_{0}-\Delta l\right) * \sin \theta+\operatorname{InTipPosX}\right] .
\end{array}
$$

It is noted that this AWG device is not of zero-order, but rather a low order regular AWG, with a constant waveguide spacing at the center of the arrayed section. The layout of a full AWG device needs to be cut into two halves. The right half of the device is then flipped horizontally. Since the waveguides have equal spacing at either sides of the "cut" interface, the flipped portion of the device can be directly connected with the original unchanged half, obtaining a true zero-order AWG device.

Based on the algorithm described here, two different approaches have been designed 
to obtain the final layout of the device. One is using the $\mathrm{C}$ programming language implemented in MATLAB ${ }^{\circledR}$. The software code includes the calculations of all the coordinates and saves them to a device structure data file (e.g., Full_AWG.scr) that can be read and plotted in a layout design program AutoCAD ${ }^{\circledR}$. The other approach uses Visual Basic ${ }^{\circledR}$ programming language, which can generate the device layout directly by loading in a phasar design software Optiwave ${ }^{\circledR}$. Both approaches have been used to verify the design specifications and accuracies.

\section{B.1 MATLAB ${ }^{\circledR}$ Programming Code}

Simulation MATLAB ${ }^{\circledR}$ code for generating our AWG device with equal vertical waveguide spacing in the center of the arrayed section:

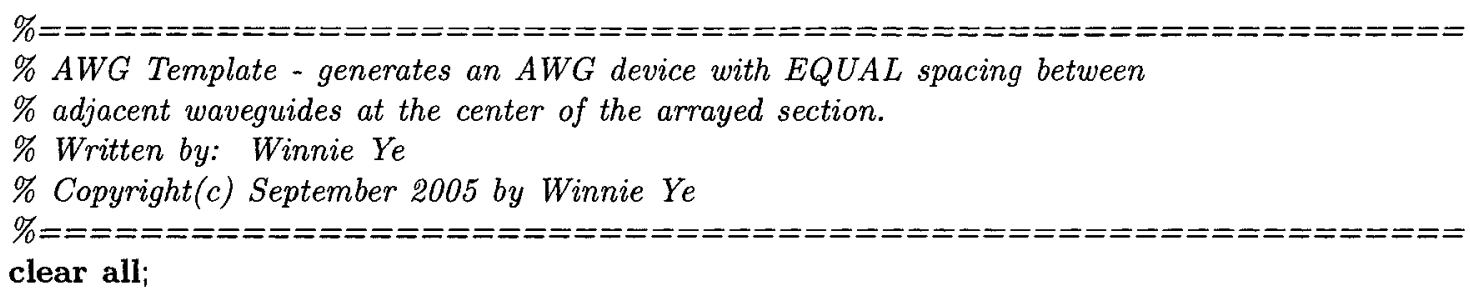


ChannelLen $=[]_{i} \quad \%$ Total channel length of the waveguides in the arrayed section

Coordinates $=[] ; \quad \%$ Starting and ending cooridnates of the curved waveguides in the arrayed section 30

$\mathrm{Rad}=[] ; \quad \%$ Radius of the curved waveguides in the arrayed section

Output_l=[]; \% Cooridinate matrix of the left side of the $A W G$

Output_r $=[] ; \quad \%$ Cooridinate matrix of the right side of the $A W G$

Output_full=[]; \% Cooridinate matrix of the FULL AWG

$\%$ The loop calculates the length, location, of the waveguides in the

$\%$ arrayed section

for $\mathrm{iPA}=1: 1:$ NumberOfPAwgs

ThetaPA =OrientationAngle+AngleIncrement* $(\mathrm{iPA}-1-($ NumberOfPAwgs -1$) / 2)$;

Alpha $=\mathrm{pi}() / 2-$ ThetaPA $/ 2 ; \quad \%$ Orientation angle of the specific waveguide

$\mathrm{Xr}=$ CenterPA_X+(iPA-1)*ConstSep-500; \% Minimum band radius is $500 u m$

$10=\operatorname{abs}([(\mathrm{PA}-1) *$ ConstSep+CenterPA_X-InTipPosX] $] / \sin ($ ThetaPA $)$;

Error $=1$;

while (abs(Error) $>0.001) \%$ Error in length is set to be less than $1 \mathrm{~nm}$

\% Calculation of the center coordinates of the curved

$\%$ waveguides $(Z r, X r)$

$\mathrm{Zr}=-(\mathrm{Xr}-\operatorname{In} \operatorname{TipPos} \mathrm{X}) / \tan (\mathrm{Alpha})+10+\operatorname{InTipPosZ}$;

if $(\mathrm{Zr}<=$ CenterPA_Z)

$\%$ Calculate the length 11

$11=10-\operatorname{abs}(((\mathrm{PA}-1) *$ ConstSep+CenterPA_X-Xr)/tan(Alpha) $)$;

$\%$ min requirement: $100 u m$ for tapers and $100 u m$ for straight

$\%$ waveguides for $l 1$

if $(11>=$ CouplerLength +200$)$

arcLength $=((\mathrm{iPA}-1) *$ ConstSep + CenterPA_X $-\mathrm{Xr}) *$ ThetaPA

Totallength=abs(CenterPA_Z-Zr) +abs(11)+abs(arcLength);

Error $=$ TotalLength $-($ TipSeparation + InitialLengthIncrement. . . +LengthIncrement*(iPA-1))/2;

else

display('Error: Cannot find a circle with given tangents');

break;

else

display('Error: BAD TipSeparation');

break;

end

$\mathrm{Xr}=\mathrm{Xr}-0.01$

\%Error

end

$\operatorname{Rad}=[\operatorname{Rad} ;[\operatorname{arcLength} /$ ThetaPA $\mathrm{Zr} \mathrm{Xr}+0.1$ ThetaPA]]; \%Radius, CenterZ, CenterX, angle

ChannelLen=[ChannelLen; [11 arcLength CenterPA_Z-Zr TotalLength]];

Coordinates $=[$ Coordinates; $[$ InTipPosZ $+11 * \cos ($ ThetaPA $)$ InTipPosX $+11 * \sin ($ ThetaPA $) ..$

$\mathrm{Zr}(\mathrm{iPA}-1)^{*}$ ConstSep+CenterPA_X]];

$\%$ Saves the coordinates of the left $A W G$ structure

Output_l=[Output_l; [CenterPA_Z CenterPA_X+(iPA-1)*ConstSep Zr ...

CenterPA_X+(iPA-1)*ConstSep arcLength/ThetaPA InTipPosZ+11*cos(ThetaPA)...

InTipPosX+11*sin(ThetaPA) InTipPosZ+(CouplerLength+200)* $\cos ($ ThetaPA ) .. .

InTipPosX $+($ CouplerLength +200$) * \sin ($ ThetaPA $) \ldots$

InTipPosZ+(CouplerLength +100$) * \cos ($ ThetaPA $) \ldots$

InTipPosX $+(\text { CouplerLength }+100)^{*} \sin ($ ThetaPA $) \ldots$

InTipPosZ+CouplerLength* $\cos$ (ThetaPA). .

[iPA Error] InTipPosX+CouplerLength*sin(ThetaPA)]]; 
end

$\%$ Plot the left half of the AWG in MATLAB for visualization

factor $=50 ; \%$ Total number of plotted data points

figure;

hold on;

$\%$ Plot the straight waveguide section in the FPR region

for $i=1: 1$ :size(Coordinates, 1 )

slope $=($ Coordinates $(\mathbf{i}, 2)-\operatorname{InTipPosX}) /(\operatorname{Coordinates}(\mathbf{i}, \mathbf{1})-\operatorname{InTipPosZ})$;

end

$\mathrm{zZ}=$ InTipPosZ:(Coordinates(i,1)-InTipPosZ)/factor:Coordinates $(\mathbf{i}, \mathbf{1})$;

$\mathrm{xx}=\operatorname{InTipPos} \mathrm{X}+$ slope $^{*}(\mathrm{zz}-\operatorname{InTipPosZ})$;

$\operatorname{plot}\left(z z, x x_{1}{ }^{\prime} g\right.$ ');

$\%$ Plot the straight waveguides (variable waveguide length) in the arrayed

$\%$ waveguide section

for $\mathbf{i}=1: 1$ :size(Coordinates, 1 )

$\mathrm{zz}=$ Coordinates $(\mathbf{i}, 3):($ CenterPA_Z_Coordinates $(\mathbf{i}, 3)) /$ factor:CenterPA_Z;

$\mathrm{xx}=$ Coordinates $(\mathbf{i}, 4)^{*}$ ones $(\operatorname{size}(\mathrm{zz}))$;

end $\operatorname{plot}\left(\mathrm{zz}, \mathrm{xx},{ }^{\prime} \mathrm{b}\right.$ ');

$\%$ Plot the curved waveguides in the arrayed waveguide section

for $j=1: 1: \operatorname{size}(\operatorname{Rad}, 1)$

$\mathrm{P} 1 \mathrm{i}=$ Coordinates $(\mathbf{j}, \mathbf{1}):(\operatorname{Coordinates}(\mathbf{j}, 3)-\operatorname{Coordinates}(\mathbf{j}, \mathbf{1})) /$ factor:Coordinates $(\mathbf{j}, 3)$;

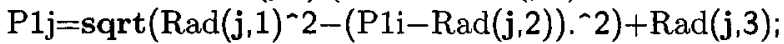

end $\operatorname{plot}\left(\mathrm{P} 1 \mathrm{i}, \mathrm{P} 1 \mathrm{j},{ }^{\prime} \mathrm{r}^{\prime}\right)$

hold off;

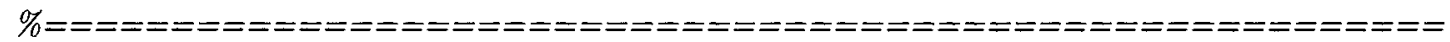
$\%$ Prepare for the final layout of a complete zero-order $A W G$ with tapered $\%$ waveguides, straight and curved waveguides

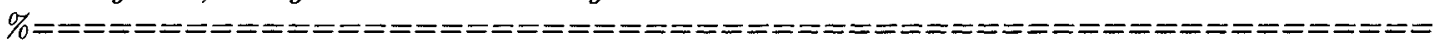

\% The right half $A W G$ is a perfect image of the left side.

for $\mathbf{i}=1: 1: 100$ end

Output_r=[Output_r; Output_l( $\left.\left.100-\mathbf{i}+\mathbf{1}_{1}:\right)\right]$;

$\%$ Reformat the Output_r to obtain the coordinates of the right half of the

$\% A W G$

$\mathrm{a}=$ Output_r;

$a(: 1)=$ Output_r $(100,1)-a(:, 1)+$ Output_l $(1,1)$;

$a(:, 3)=$ Output_r $(100,1)-a(:, 3)+$ Output_l $(1,1)$;

$\mathrm{a}(:, 6)=$ Output_r $(100,1)-\mathrm{a}(:, 6)+$ Output_l $(1,1)$;

$\mathrm{a}(:, 8)=$ Output_r $(100,1)-\mathrm{a}(:, 8)+$ Output_l$(1,1)$;

$\mathrm{a}(:, 10)=$ Output_r $(100,1)-\mathrm{a}(:, 10)+$ Output_l $(1,1)$;

$\mathrm{a}(:, 12)=$ Output_r $(100,1)-\mathrm{a}(:, 12)+$ Output_l $(1,1)$;

$\mathrm{a}(:, 2)=2 *$ Output_r $(100,2)+$ ConstSep $*$ (NumberOfPAwgs -1$)-\mathrm{a}(:, 2)$;

$a(:, 4)=2^{*}$ Output_r $(100,2)+$ ConstSep $*$ (NumberOfPAwgs-1)-a $(:, 4)$;

$\mathrm{a}(:, 7)=2^{*}$ Output_r $(100,2)+$ ConstSep* ${ }^{*}$ NumberOfPAwgs-1)-a $(:, 7)$;

$\mathrm{a}(:, 9)=2 *$ Output_r $(100,2)+$ ConstSep*(NumberOfPAwgs-1)-a(:,9);

$\mathrm{a}(:, 11)=2 *$ Output_r $(100,2)+$ ConstSep*(NumberOfPAwgs-1) $-\mathrm{a}(:, 11)$;

$a(:, 13)=2 *$ Output_r $(100,2)+$ ConstSep*(NumberOfPAwgs -1$)-a(:, 13)$;

Output_r=a; 
\% Combine the left and right sides of the AWG

Output_full=[Output_l Output_r];

\% Save Output matrix to a structure data file that can be read by AutoCAD

fid =fopen('Full_AWG. $s c r^{\prime}$, 'w');

$\%$ waveguide width is set to $2 u m$ here as an example.

fprintf(fid,'pline $\% 1.9 e, \% 1.9 e$ w $22 \% 1.9 e, \% 1.9 e$ a $\times \% 1.9 e \% 1.9 e, \% 1.9 e 1 \% 1.9 e, \% 1.9 e$

fclose(fid);

w $22 \% 1.9 e, \% 1.9 e$ w $23.5 \% 1.9 e, \% 1.9 e \backslash r^{\prime}$, Output_full');

\section{B.2 Visual Basic ${ }^{\circledR}$ Programming Code}

Simulation Visual Basic ${ }^{\circledR}$ code for generating our AWG device with equal vertical waveguide spacing in the center of the arrayed section:

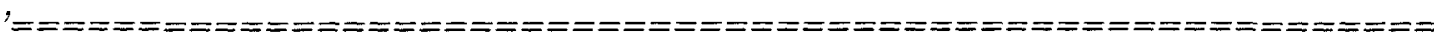

' $A W G$ Template - generates an $A W G$ device with $E Q U A L$ spacing between adjacent

, waveguides at the center of the arrayed section.

' Created by: Winnie Ye in September 2005

' (based on a sample code provided by Optiwave)

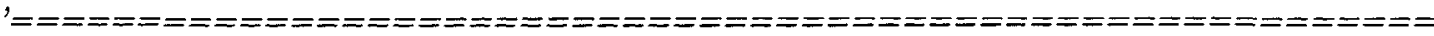

'Draw the $A W G$ layout according to the $A W G$ parameters listed below

Option Explicit

WgMgr.DeleteAll()

Dim IOWgSeparation, CouplerLength, PAWgLength, Pi, TipSeparation, InTipPosX Dim InTipPosZ, LengthIncrement, IOWgLength, AngularWidth, WaveguideWidth Dim InitialLengthIncrement, OrientationAngle, NumberOfPAwgs, PAWgSeparation Dim IONumber, PortSeparation, ConnectionOffset, WaferLength, WaferWidth

Dim ConstSep, CenterPA_X, CenterPA_Z

$\mathrm{Pi}=3.14159$

' $A W G$ Parameters

TipSeparation $=8970^{\prime}$ Separation between star coupler tips

InTipPosX $=-1100^{\prime}$ 'Vertical coordinate of the tip of input star coupler InTipPosZ $=2000$ 'Horizontal coordinate of the tip of input star coupler

LengthIncrement $=9{ }^{\prime}$ Difference in path length of adjacent waveguides in 'the Phased Array

InitialLengthIncrement $=377$ 'Distance added to the first path in the Phased Array

OrientationAngle $=30^{\prime}$ Angle of inclination of the star couplers from the horizontal NumberOfPAwgs $=100^{\prime}$ Number of waveguides in Phased Array 


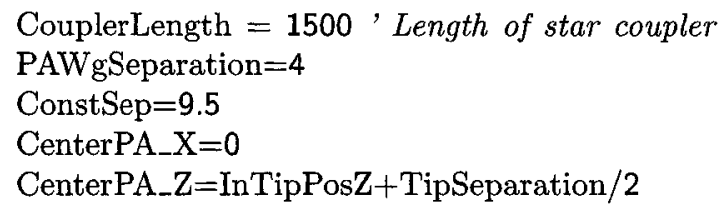

IOWgSeparation $=4.4^{\prime}$ Minimum waveguide separation in $I / O$ waveguide array PAWgLength $=200$ ' Length of waveguides on PA side of star coupler IOWgLength $=400^{\prime}$ Length of waveguides on I/O side of star coupler AngularWidth $=30.0^{\prime}$ 'Angle specifying width of the star couplers IONumber $=17$ ' Number of waveguides in $I / O$ Array

PortSeparation $=50$ 'Distance between adjacent ports at the wafer edge

ConnectionOffset $=0$ 'Vertical distance between wafer edge and first port

WaferLength $=15029$ ' Length of the wafer (horizontal)

WaferWidth $=4350^{\prime}$ 'Width of the wafer (vertical)

WaveguideWidth $=2$, Width of all waveguides

'Specify a waveguide design called WaveguideGeo1

Dim WaveguideGeo1, result, error, Tol

Set WaveguideGeo1 = New WaveguideGeometry WaveguideGeol.Initialize()

WaveguideGeo1.mWidth $=$ WaveguideWidth

WaveguideGeo1.mThickness $=0.5$

WaveguideGeol.mDepth $=0.0$

WaveguideGeol.mProfileName $=$ "ChannelPro1"

' Specify AWG Phased Array parameters

Dim PhasedArray

Set PhasedArray $=$ New SA_Template

PhasedArray.mTipSeparation $=$ TipSeparation

PhasedArray.mInTipPosX $=$ InTipPosX

PhasedArray.mInTipPosZ $=$ InTipPosZ

PhasedArray.mLengthIncrement $=$ LengthIncrement

PhasedArray.mInitialLengthIncrement $=$ InitialLengthIncrement

PhasedArray.mOrientationAngle $=\mathrm{Pi}$ * OrientationAngle $/ 180$.

PhasedArray.mAngleIncrement = PAWgSeparation / CouplerLength

PhasedArray.mNumberOfPAwgs $=$ NumberOfPAwgs

PhasedArray $\cdot$ mEndRadius = CouplerLength + PAWgLength

PhasedArray.mConstSep $=$ ConstSep

PhasedArray.mCenterPA_X $=$ CenterPA_X

PhasedArray.mCenterPA_Z = CenterPA_Z

'Draw the phased array

PhasedArray.Initialize(WaveguideGeo1)

PhasedArray.Create("PA1")

'Specify Input Star Coupler parameters

Dim Coupler

Set Coupler $=$ New Star

Coupler.Initialize(WaveguideGeo1)

Coupler.mOrientationAngle $=\mathrm{Pi} *(180-$ OrientationAngle $) / 180$.

Coupler.mTipPositionX $=$ InTipPosX

Coupler.mTipPositionZ = InTipPosZ

Coupler.mPAWgLength $=$ PAWgLength 
Coupler.mIOWgLength $=$ IOWgLength

Coupler.mAngularWidth $=\mathrm{Pi}^{*}$ AngularWidth $/ 180$.

Coupler.mCouplerLength $=$ CouplerLength

Coupler.mPANumber $=$ NumberOfPAwgs

Coupler.mIONumber $=$ IONumber

Coupler.mIOWgSeparation = IOWgSeparation

Coupler.mPAWgSeparation $=$ PAWgSeparation

' Draw input star coupler

Coupler.Create("InputStar")

'Specify Output Star Coupler parameters and draw it

Coupler.mTipPositionZ = InTipPosZ + TipSeparation

Coupler.mOrientationAngle $=\mathrm{Pi}^{*}$ OrientationAngle $/ 180$.

Coupler.Create("OutputStar")

' Specify input array and draw it

Dim IOArray

Set IOArray $=$ New Input_Array

IOArray.Initialize(WaveguideGeo1)

IOArray.mArrayCentreX $=$ InTipPosX +

CouplerLength $* \operatorname{Sin}(\mathrm{Pi} *$ OrientationAngle / 180.)

IOArray.mArrayCentreZ = InTipPosZ +

CouplerLength * $\operatorname{Cos}(\mathrm{Pi} *$ OrientationAngle / 180.)

IOArray. OrientationAngle $=\mathrm{Pi} *$ OrientationAngle $/ 180$.

IOArray.mAngleIncrement = IOWgSeparation $/$ CouplerLength

IOArray.mNumberOfIOwgs $=$ IONumber

IOArray $\cdot$ mIORadius $=$ CouplerLength + IOWgLength

IOArray $\cdot$ mPortSeparation $=$ PortSeparation

IOArray.mConnectionOffset $=$ ConnectionOffset

IOArray.mWaferWidth $=$ WaferWidth

IOArray $\cdot$ mWaferLength $=$ WaferLength

IOArray.Create("InputArray")

' Specify output array and draw it

Set IOArray $=$ New Output_Array

IOArray.Initialize(WaveguideGeo1)

IOArray.mArrayCentreX $=$ InTipPosX +

CouplerLength * $\operatorname{Sin}\left(\mathrm{Pi}^{*}\right.$ OrientationAngle / 180.)

IOArray mOrientationAngle $=\mathrm{Pi} *(180.0-$ OrientationAngle $) / 180.0$

IOArray $\cdot \mathrm{mArrayCentreZ}=\operatorname{InTipPosZ}+$ TipSeparation -

CouplerLength * $\operatorname{Cos}(\mathrm{Pi} *$ OrientationAngle / 180.)

IOArray.mAngleIncrement $=$ IOWgSeparation / CouplerLength

IOArray.mNumberOfIOwgs $=$ IONumber

IOArray.mIORadius $=$ CouplerLength + IOWgLength

IOArray .mPortSeparation $=$ PortSeparation

IOArray.mConnectionOffset $=$ ConnectionOffset

IOArray.mWaferWidth $=$ WaferWidth

IOArray.mWaferLength $=$ WaferLength

IOArray.Create("OutputArray") 
' End of main program

Class SA_Template

Dim SA_ID 'ID to make waveguide names unique

Dim mTipSeparation 'Separation between star coupler tips

Dim mInTipPosX 'Vertical coordinate of the tip of input star coupler

Dim mInTipPosZ' Horizontal coordinate of the tip of input star coupler

Dim mLengthIncrement 'Diff in path length of adjacent waveguides

' in the Phased Array

Dim mInitiallengthIncrement 'Distance added to the first path in the

'Phased Array

Dim mOrientationAngle 'Angle of inclination of the star couplers

' from the horizontal

Dim mAngleIncrement 'Angle between $P A$ waveguides

Dim mNumberOfPAwgs ' Number of waveguides in Phased Array

Dim mEndRadius 'CouplerLength + PAWgLength

Dim mWaveguideGeo ' WaveguideGeometry object holding waveguide details

Dim mConstSep 'Cosntant separation in the center of the PA section

Dim mCenterPA_X, mCenterPA_Z' Coordinates of the center of the bottom ' waveguide in the $P A$ section

Sub Initialize(WaveguideGeo)

Set $m$ WaveguideGeo $=$ New WaveguideGeometry

mWaveguideGeo.Initialize

mWaveguideGeo.mWidth $=$ WaveguideGeo $m$ Width

mWaveguideGeo.mThickness $=$ WaveguideGeo.mThickness

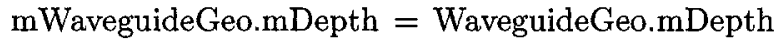

$\mathrm{mWaveguideGeo \cdot mProfileName}=$ WaveguideGeo.mProfileName

mWaveguideGeo.mRadiusUsed = WaveguideGeo.mRadiusUsed

$\mathrm{mWaveguideGeo \cdot mRadiusType}=$ WaveguideGeo.mRadiusType

$\mathrm{mWaveguideGeo \cdot mNewPosBase}=$ WaveguideGeo $m$ NewPosBase

$\mathrm{mWaveguideGeo \cdot mAngleOffset}=$ WaveguideGeo.mAngleOffset

End Sub

Sub Create(SA_ID)

Dim iPA, ThetaPA, PA_Radius, PA_x, oLinear, oArc, result, X1, Z1

Dim X2, Z2, PathLength, arcLength, TotalLength

Dim 10, 11, Xr, Zr, Alpha, Error

For $\mathrm{iPA}=1$ to $\mathrm{mNumberOfPAwgs}$

ThetaPA $=$ mOrientationAngle + mAngleIncrement $*$

(iPA - $1-(\mathrm{mNumberOfPAwgs}-1) / 2.0)$

Alpha $=\mathrm{Pi} / 2-\mathrm{ThetaPA} / 2$

$\mathrm{Xr}=\mathrm{mCenterPA} \mathrm{X}+(\mathrm{iPA}-1) * \mathrm{mConstSep}-500$ 'minimum band radius

Do

Error $=1$

'MsgBox("While loop ran")

$\mathrm{Zr}=-(\mathrm{Xr}-\mathrm{mInTipPos} \mathrm{X}) / \tan ($ Alpha $)+10+\mathrm{mInTipPosZ}$

If $(\mathrm{Zr}<=\mathrm{mCenterPA} Z \mathrm{Z})$ Then

$11=10-\operatorname{abs}\left(\left((\mathrm{PA}-1)^{*} \mathrm{mConstSep}+\mathrm{mCenterPA} \mathrm{X}-\mathrm{Xr}\right) / \mathrm{Tan}(\mathrm{Alpha})\right)$

If (11>=mEndRadius) Then

arcLength $=\left((\mathrm{PPA}-1)^{*} \mathrm{mConstSep}+\mathrm{mCenterPA} \mathrm{X}-\mathrm{Xr}\right)^{*}$ ThetaPA

TotalLength=abs (mCenterPA_Z-Zr) + abs(11)+abs(arcLength)

Error $=$ TotalLength $-(\mathrm{mTipSeparation}+$ mInitialLengthIncrement 
End If

$$
+ \text { mLengthIncrement*(iPA-1))/2 }
$$

End If

$\mathrm{Xr}=\mathrm{Xr}-0.1$

Loop Until (abs(Error) $<=0.1$ )

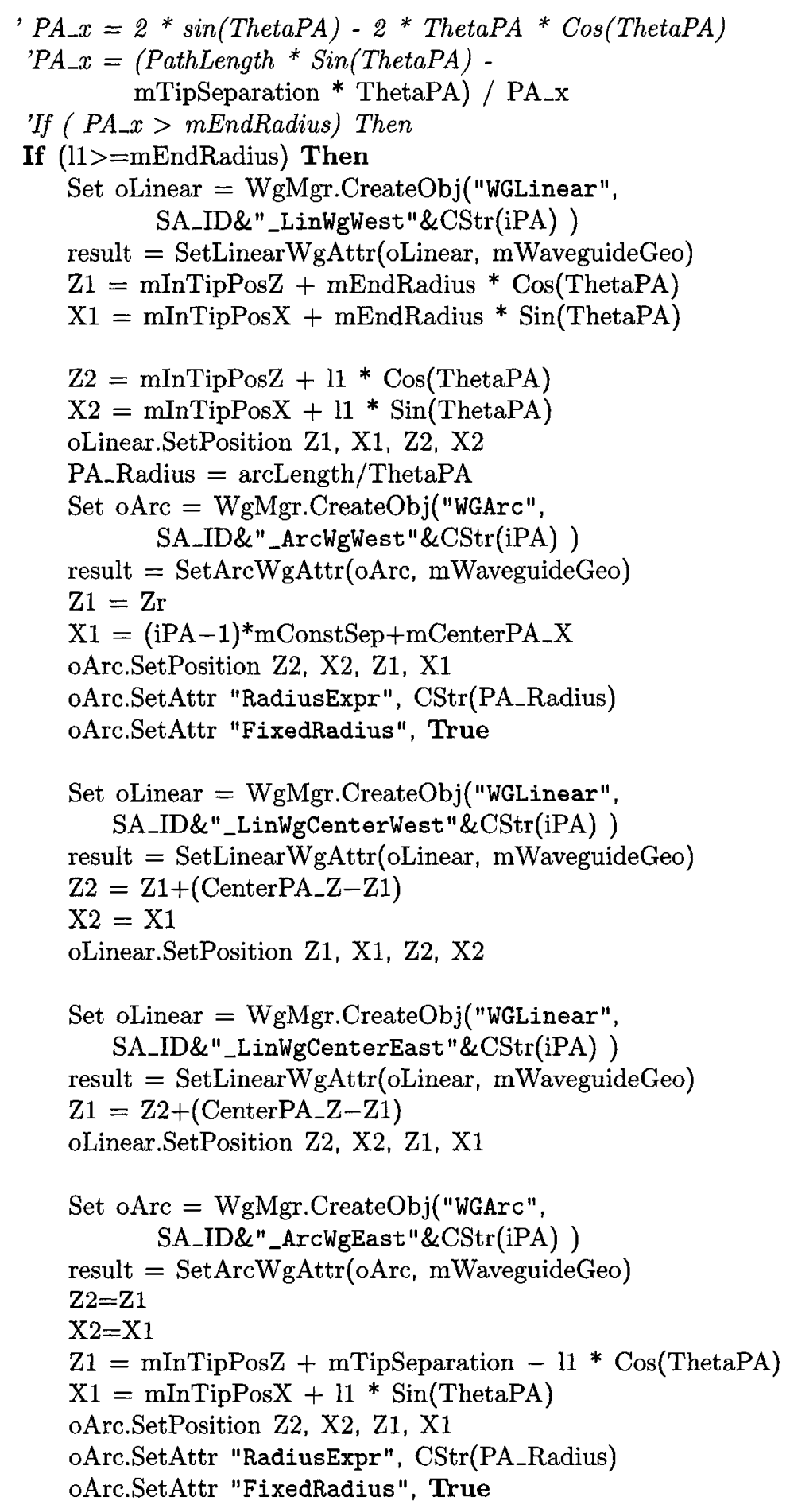


Set oLinear $=$ WgMgr.CreateObj("WGLinear", SA_ID\&"_LinWgEast"\&CStr(iPA) )

result $=$ SetLinearWgAttr(oLinear, mWaveguideGeo)

$\mathrm{Z} 2=$ mInTipPosZ + mTipSeparation - mEndRadius $*$ Cos(ThetaPA $)$

$\mathrm{X} 2=$ mInTipPos $\mathrm{X}+$ mEndRadius $* \operatorname{Sin}($ ThetaPA $)$

oLinear.SetPosition $\mathrm{Z} 1, \mathrm{X} 1, \mathrm{Z} 2, \mathrm{X} 2$

\section{End If}

Next

End Sub' end Create subroutine

End Class ' end of class SA_Template

'Set the properties of the linear waveguide object oLinear according to

' those found in class WaveguideGeo

Function SetLinearWgAttr(oLinear, WaveguideGeo)

oLinear.SetAttr "WidthExpr", CStr(WaveguideGeo.mWidth)

oLinear.SetAttr "Depth", CStr(WaveguideGeo.mDepth)

oLinear.SetAttr "StartThickness", CStr(WaveguideGeo.mThickness)

oLinear.SetAttr "EndThickness",CStr(WaveguideGeo.mThickness )

oLinear.SetProfileName WaveguideGeo.mProfileName

oLinear.SetDefaultThickness TaperMode False

oLinear.SetThicknessTaperType 1

SetLinearWgAttr = True

\section{End Function}

'Set the properties of the arc waveguide object oArc according to

'those found in class WaveguideGeo

Function SetArcWgAttr(oArc, WaveguideGeo)

oArc.SetAttr "WidthExpr", CStr(WaveguideGeo.mWidth)

oArc.SetAttr "Depth", CStr(WaveguideGeo.mDepth )

oArc.SetAttr "StartThickness", CStr(WaveguideGeo.mThickness )

oArc.SetAttr "EndThickness",CStr(WaveguideGeo.mThickness )

oArc.SetProfileName WaveguideGeo.mProfileName

oArc.SetAttr "RadiusExpr", "O"

oArc.SetAttr "FixedRadius", False

oArc.SetAttr "BoundaryDirection", True

oArc.SetDefaultThickness TaperMode True

SetArcWgAttr = True

\section{End Function}

' Class to hold all attributes of any kind of waveguide

Class WaveguideGeometry

Dim mWidth

Dim mThickness

Dim mDepth

Dim mProfileName

Dim mRadiusUsed

Dim mRadiusType

Dim mNewPosBase 
Dim mAngleOffset

'Use this method to set default values for all attributes

' (like a constructor)

Sub Initialize()

$\mathrm{mWidth}=4.0$

mThickness $=2.0$

$\mathrm{mDepth}=0.0$

mProfileName $=$ "ChannelPro1"

mRadius Used $=$ False

mRadiusType $=0$

$\mathrm{mNewPosBase}=0$

mAngleOffset $=0.0$

End Sub

\section{End Class}

' Class to create a Free Propagation Region Coupler in an AWG

Class Star

Dim mFPR_ID 'ID to make waveguide names unique

Dim mPhased_Array ' RadialArray object for array on the phased array side

Dim mIO_Array ' RadialArray object for array on the $I / O$ waveguide array side

Dim mOrientationAngle' Angle of inclination of Star coupler to $Z$ axis

Dim mAngularWidth 'Angular width of FPR

Dim mCouplerLength ' Length of Star coupler

Dim mWaveguideGeo 'Profile geometry of waveguides used in the

' waveguide array and coupler

Dim mTipPositionZ ' $Z$ coordinate of tip of Star coupler

Dim mTipPositionX ' $X$ coordinate of tip of Star coupler

Dim mPAWgLength 'Length of waveguides on phased array side

Dim mPAWgSeparation 'Distance between PA waveguide centres at junction

Dim mPANumber 'Number of waveguides on phased array side

Dim mIOWgLength 'Length of waveguides on $I / O$ array side

Dim mIOWgSeparation 'Distance between $I / O$ waveguide centres at junction

Dim mIONumber 'Number of waveguides on $I / O$ array side

Sub Initialize(WaveguideGeo)

$$
\begin{aligned}
& \text { mFPR_ID }=0 \\
& \text { mOrientationAngle }=0.0 \\
& \text { mAngularWidth }=0.2 \\
& \text { mCouplerLength }=250.0 \\
& \text { mTipPositionZ }=1200.0 \\
& \text { mTipPositionX }=0.0 \\
& \text { mPAWgLength }=750.0 \\
& \text { mPAWgSeparation }=5.0 \\
& \text { mPANumber }=15 \\
& \text { mIOWgLength }=200 . \\
& \text { mIOWgSeparation }=\text { mPAWgSeparation }
\end{aligned}
$$$$
\text { mIONumber }=4
$$

Set $\mathrm{mWaveguideGeo}=$ New WaveguideGeometry

mWaveguideGeo.Initialize

$\mathrm{mWaveguideGeo.mWidth}=$ WaveguideGeo.mWidth

mWaveguideGeo.mThickness $=$ WaveguideGeo.mThickness 
$\mathrm{mWaveguideGeo \cdot mDepth}=$ WaveguideGeo.mDepth $\mathrm{m}$ WaveguideGeo.mProfileName $=$ WaveguideGeo.mProfileName mWaveguideGeo.mRadiusUsed $=$ WaveguideGeo.mRadiusUsed mWaveguideGeo.mRadiusType $=$ WaveguideGeo.mRadiusType $\mathrm{mWaveguideGeo \cdot mNewPosBase}=$ WaveguideGeo.mNewPosBase mWaveguideGeo.mAngleOffset $=$ WaveguideGeo.mAngleOffset Set $m$ Phased_Array $=$ New RadialArray

Set mIO_Array $=$ New RadialArray

\section{End Sub}

Sub Create(CouplerID)

mFPR_ID $=$ CouplerID

' Create Phased Array waveguides

mPhased_Array.Initialize(mWaveguideGeo)

$\mathrm{mPhased \_ Array.mNumberOfWg}=\mathrm{mPANumber}$

$\mathrm{mPhased \_ Array} \cdot \mathrm{mInnerRadius}=\mathrm{mCouplerLength} / 2$

mPhased_Array $\cdot$ mOuterRadius $=$ mPAWgLength + mCouplerLength

Dim angle

angle $=($ mPAWgSeparation $/$ mCouplerLength $) *($ mPANumber -1$) / 2$

mPhased_Array.mStartAngle $=\mathrm{Pi}+$ angle - mOrientationAngle

mPhased_Array $\cdot \mathrm{mEndAngle}=\mathrm{Pi}-$ angle - mOrientationAngle

$\mathrm{mPhased}$ _Array $\cdot \mathrm{mXCentre}=\mathrm{mTipPositionX}$

mPhased_Array. $\mathrm{mZCentre}=\mathrm{mTipPositionZ}$

mPhased_Array.Create(mFPR_ID\&"_PAwg")

' Create I/O Array waveguides

mIO_Array.Initialize(mWaveguideGeo)

mIO_Array.mNumberOfWg $=$ mIONumber

mIO_Array.mInnerRadius $=\mathrm{mCouplerLength} / 2$

mIO_Array.mOuterRadius $=$ mIOWgLength + mCouplerLength

angle $=($ mIOWgSeparation $/$ mCouplerLength $) *($ mIONumber -1$) / 2$

mIO_Array.mStartAngle $=+$ angle - mOrientationAngle

mIO_Array.mEndAngle $=-$ angle - mOrientationAngle

mIO_Array.mXCentre $=\mathrm{m}$ TipPositionX + mCouplerLength $*$ $\operatorname{Sin}(m$ OrientationAngle)

mIO_Array.mZCentre $=\mathrm{mTipPositionZ}-\mathrm{mCouplerLength}$ * $\operatorname{Cos}($ mOrientationAngle)

mIO_Array.Create(mFPR_ID\&"_IOwg")

' Draw the circular end of the coupler on PA side

Dim Circ

Set Circ $=$ WGMgr.CreateObj("WGLensCircular",mFPR_ID\&"_PA")

Circ.SetStart mTipPositionZ, mTipPositionX

Circ.SetAttr "RadiusOffset", CDbl(mCouplerLength)

Circ.SetAttr "RightClipped",true

Circ.SetAttr "ClipRightoffset", CDbl(mCouplerLength)

Circ.SetAttr "ClipLeftoff set", CDbl (mCouplerLength *

Circ.SetAttr "LeftClipped",true cos(mAngularWidth / 2.0))

Circ.SetAttr "ClipUpOffset", CDbl(mCouplerLength)

Circ.SetAttr "UpClipped",true

Circ.SetAttr "ClipDownOff set", CDbl(-mCouplerLength)

Circ.SetAttr "DownClipped", true 


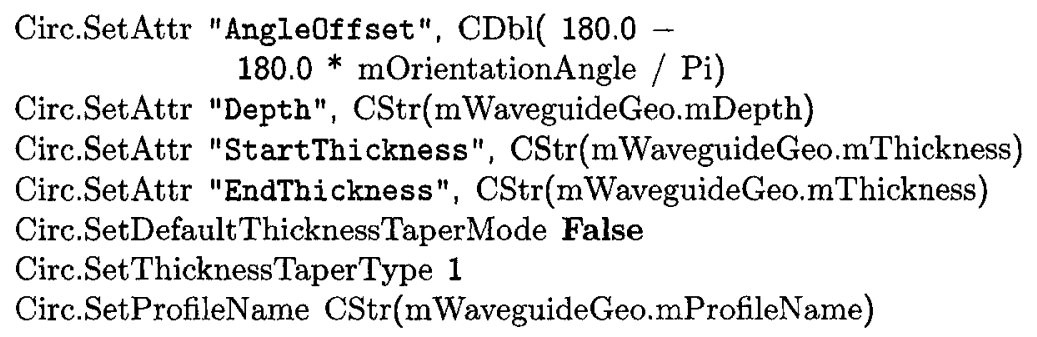

'Draw the rectangular part of the coupler

Dim FPR, Halfwidth, FPR1, FPR2,Z1, Z2, X1, X2

Set $\mathrm{FPR}=$ WgMgr.CreateObj("WGLinear",mFPR_ID\&"_FPR")

result $=$ SetLinearWgAttr(FPR, mWaveguideGeo)

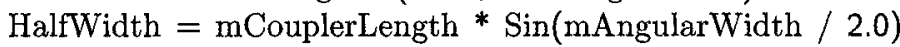

FPR.SetAttr "WidthExpr", CStr(Halfwidth * 2.0)

FPR.SetAttr "StartThickness", CStr(mWaveguideGeo.mThickness)

FPR.SetAttr "EndThickness", CStr(mWaveguideGeo.mThickness)

FPR.SetDefaultThicknessTaperMode False

FPR.SetThicknessTaperType 1

FPR1 $=$ mCouplerLength $*$ Cos (mAngularWidth / 2.0)

FPR2 $=$ mCouplerLength $/ 2.0-\operatorname{sqr}\left(\right.$ mCouplerLength ${ }^{\wedge} 2 / 4.0-$ HalfWidth^2)

$\mathrm{Z} 1=$ mTipPositionZ - FPR1 * Cos(mOrientationAngle $)$

$\mathrm{X} 1=\mathrm{mTipPositionX}+\mathrm{FPR} 1 * \operatorname{Sin}(\mathrm{m}$ OrientationAngle)

$\mathrm{Z} 2=$ mTipPositionZ - FPR2 $* \operatorname{Cos}($ mOrientationAngle $)$

$\mathrm{X} 2=\mathrm{mTipPositionX}+\mathrm{FPR} 2 * \operatorname{Sin}(\mathrm{m}$ OrientationAngle)

FPR.SetPosition Z1, X1, Z2, X2

'Draw the circular end of the coupler on $I / O$ side

Set Circ = WGMgr.CreateObj("WGLensCircular",mFPR_ID\&"_IO")

$\mathrm{Z} 1=\mathrm{m}$ TipPositionZ - mCouplerLength $* \operatorname{Cos}($ mOrientationAngle) $/ 2.0$

$\mathrm{X} 1=\mathrm{mTipPositionX}+\mathrm{m}$ CouplerLength $* \operatorname{Sin}($ mOrientationAngle) $/ 2.0$

Circ.SetStart Z1, X1

Circ.SetAttr "RadiusOffset", CDbl( mCouplerLength / 2.0)

Circ.SetAttr "RightClipped",true

Circ.SetAttr "ClipRightoff set", CDbl( mCouplerLength / 2.0)

Circ.SetAttr "ClipLeftoffset", CDbl( mCouplerLength / 2.0 - FPR2 )

Circ.SetAttr "LeftClipped",true

Circ.SetAttr "ClipUpoffset", CDbl( mCouplerLength / 2.0)

Circ.SetAttr "UpClipped", true

Circ.SetAttr "ClipDownoffset", $\mathrm{CDbl}(-$ mCouplerLength / 2.0)

Circ.SetAttr "DownClipped", true

Circ.SetAttr "Angleoffset", $\mathrm{CDbl}(-180.0 *$ mOrientationAngle / Pi )

Circ.SetAttr "Depth", CStr(mWaveguideGeo.mDepth)

Circ.SetAttr "StartThickness", CStr(mWaveguideGeo.mThickness)

Circ.SetAttr "EndThickness", CStr(mWaveguideGeo.mThickness)

Circ.SetDefaultThicknessTaperMode False

Circ.SetThickness TaperType 1

Circ.SetProfileName CStr(mWaveguideGeo.mProfileName)

\section{End Sub}

End Class ' end Class Star 
' Class to create a radial array of linear waveguides

Class RadialArray

Dim mArrayID 'ID to make waveguide names unique

Dim mNumberOfWg ' Number of waveguides in the array

Dim mInnerRadius 'Radius at narrow end of waveguide array

Dim mOuterRadius ' Radius at wide end of waveguide array

Dim mStartAngle 'Angle of first waveguide in array

Dim mEndAngle 'Angle of last waveguide in array

Dim $m X$ Centre ' $X$ coordinate of common point

Dim mZCentre ' $Z$ coordinate of common point

Dim mWaveguideGeo 'Profile geometry of waveguides

Sub Initialize(WaveguideGeo)

mArrayID $=0$

$\mathrm{mXCentre}=0.0$

mZCentre $=0.0$

mNumberOfWg $=3$

mInnerRadius $=100$

mOuterRadius $=500$

mStartAngle $=-0.2$

mEndAngle $=0.2$

Set $\mathrm{mWaveguideGeo}=$ New WaveguideGeometry

mWaveguideGeo.Initialize

$\mathrm{mWaveguideGeo.mWidth}=$ WaveguideGeo.mWidth

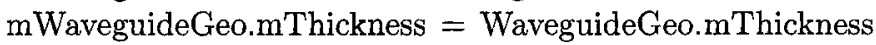

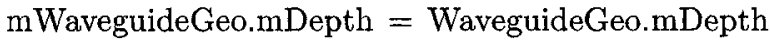

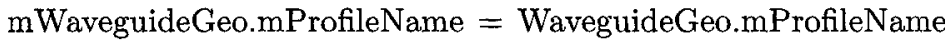

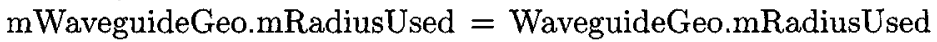

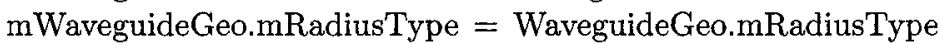
$\mathrm{m}$ WaveguideGeo.mNewPosBase $=$ WaveguideGeo.mNewPosBase $\mathrm{mWaveguideGeo \cdot mAngleOffset}=$ WaveguideGeo.mAngleOffset

End Sub

Sub Create(ArrayID)

Dim iwg, Linear, result, angle, Z1, Z2, X1, X2

mArrayID $=$ ArrayID

For iwg $=1$ to $\mathrm{mNumberOfWg}$

Set Linear = WgMgr.CreateObj("WGLinear", CStr(mArrayID)\&"_Wg"\&CStr(iwg) )

result $=$ SetLinearWgAttr(Linear, mWaveguideGeo $)$

angle $=$ mStart Angle $+($ mEndAngle - mStartAngle $) *$

(iwg - 1) / (mNumberOfWg - 1)

$\mathrm{Z} 1=$ mZCentre + mInnerRadius $*$ Cos (angle)

$\mathrm{X} 1=\mathrm{mXCentre}+$ mInnerRadius $*$ Sin(angle)

$\mathrm{Z} 2=$ mZCentre + mOuterRadius * Cos(angle)

$\mathrm{X} 2=\mathrm{mXCentre}+\mathrm{mOuterRadius} *$ Sin(angle)

Next

End Sub

End Class 'end Class RadialArray 
Class Input_Array

Dim mIO_ID

Dim mArrayCentreX

Dim mArrayCentreZ

Dim mOrientationAngle

Dim mAngleIncrement

Dim mNumberOfIOwgs

Dim mIORadius

Dim mWaveguideGeo

Dim mPortSeparation

Dim mConnectionOffset

Dim mWaferWidth

Dim mWaferLength

Sub Initialize(WaveguideGeo)

Set mWaveguideGeo $=$ New WaveguideGeometry

mWaveguideGeo.Initialize

$\mathrm{mWaveguideGeo.mWidth}=$ WaveguideGeo.mWidth

mWaveguideGeo.mThickness = WaveguideGeo.mThickness $\mathrm{mWaveguideGeo.mDepth}=$ WaveguideGeo.mDepth $\mathrm{mWaveguideGeo.mProfileName}=$ WaveguideGeo.mProfileName

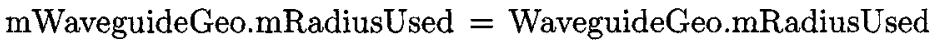
$\mathrm{mWaveguideGeo \cdot mRadiusType}=$ WaveguideGeo.mRadiusType $\mathrm{mWaveguideGeo \cdot mNewPosBase}=$ WaveguideGeo.mNewPosBase

End Sub $\mathrm{mWaveguideGeo.mAngleOffset}=$ WaveguideGeo.mAngleOffset

Sub Create(IO_ID) mIO_ID $=$ IO_ID

Dim iwg, alpha, $\mathrm{Cx}, \mathrm{Cz}, \mathrm{Ix}, \mathrm{Iz}, \mathrm{Xarc}$, Rio, Zarc, oLinear, oArc, Jz For $\mathrm{iwg}=1$ to $\mathrm{mNumberOfIOwgs}$

$$
\begin{gathered}
\text { alpha }=\text { mOrientationAngle }- \text { mAngleIncrement } * \\
(\mathrm{iwg}-1-(\mathrm{mNumberOfIOwgs}-1) / 2.0)
\end{gathered}
$$

$\mathrm{Ix}=(\mathrm{iwg}-1) *$ mPortSeparation mWaferWidth / $2.0+$ mConnectionOffset

$\mathrm{Cx}=$ mArrayCentreX - mIORadius * Sin(alpha)

$\mathrm{Xarc}=\mathrm{Cx}-\mathrm{Ix}$

Rio $=$ Xarc $/(1.0-\operatorname{Cos}($ alpha $))$

Zarc $=$ Rio $*$ Sin (alpha)

$\mathrm{Cz}=$ mArrayCentreZ - mIORadius * Cos(alpha)

Set oLinear = WgMgr.CreateObj("WGLinear",

$$
\text { CStr(IO_ID)\&"_wg"\&Cstr(iwg)) }
$$

result $=$ SetLinearWgAttr(oLinear, mWaveguideGeo)

Set oArc = WgMgr.CreateObj("WGArc",CStr(IO_ID)\&"_Arc"\&Cstr(iwg))

result $=$ SetArcWgAttr(oArc, mWaveguideGeo)

'MsgBox Rio,vbExclamation,IO_ID

oArc.SetAttr "RadiusExpr", CStr(Rio)

oArc.SetAttr "FixedRadius", True

$\mathrm{Jz}=\mathrm{Cz}-$ Zarc

$\mathrm{Iz}=0.0$

oLinear.SetPosition $\mathrm{Iz}, \mathrm{Ix}_{1} \mathrm{Jz}, \mathrm{Ix}$

oArc.SetPosition $\mathrm{Cz}, \mathrm{Cx}, \mathrm{Jz}, \mathrm{Ix}$ 


\section{Next \\ End Sub}

End Class 'end of class Input_Array

Class Output_Array

Dim mIO_ID

Dim mArrayCentreX

Dim mArrayCentreZ

Dim mOrientationAngle

Dim mAngleIncrement

Dim mNumberOfTOwgs

Dim mIORadius

Dim mWaveguideGeo

Dim mPortSeparation

Dim mConnectionOffset

Dim mWaferWidth

Dim mWaferLength

Sub Initialize(WaveguideGeo)

Set $\mathrm{mWaveguideGeo}=$ New WaveguideGeometry

mWaveguideGeo.Initialize

mWaveguideGeo.mWidth = WaveguideGeo.mWidth

mWaveguideGeo.mThickness = WaveguideGeo.mThickness

mWaveguideGeo.mDepth $=$ WaveguideGeo $\cdot \mathrm{mDepth}$

mWaveguideGeo.mProfileName $=$ WaveguideGeo.mProfileName

mWaveguideGeo.mRadiusUsed $=$ WaveguideGeo.mRadiusUsed

mWaveguideGeo.mRadiusType $=$ WaveguideGeo.mRadiusType

mWaveguideGeo.mNewPosBase $=$ WaveguideGeo.mNewPosBase

$\mathrm{mWaveguideGeo} \cdot \mathrm{mAngleOffset}=$ WaveguideGeo.mAngleOffset

End Sub

Sub Create(IO_ID)

mIO_ID $=$ IO_ID

Dim iwg, alpha, $\mathrm{Cx}, \mathrm{Cz}, \mathrm{Ix}, \mathrm{Iz}$, Xarc, Rio, Zarc, oLinear, oArc, Jz

For iwg $=1$ to mNumberOfIOwgs

alpha $=$ mOrientationAngle + mAngleIncrement ${ }^{*}$

$($ iwg $-1-($ mNumberOfIOwgs -1$) / 2.0)$

$\mathrm{Ix}=(\mathrm{iwg}-1) *$ mPortSeparation -

mWaferWidth / $2.0+$ mConnectionOffset

$\mathrm{Cx}=\mathrm{mArrayCentreX}-$ mIORadius $* \operatorname{Sin}($ alpha $)$

$\mathrm{Xarc}=\mathrm{Cx}-\mathrm{Ix}$

Rio $=\operatorname{Xarc} /(1.0+\operatorname{Cos}($ alpha $))$

Zarc $=$ Rio ${ }^{*}$ Sin(alpha)

$\mathrm{Cz}=$ mArrayCentre $Z$ - mIORadius * Cos(alpha)

Set oLinear = WgMgr.CreateObj("WGLineax",

CStr(IO_ID)\&"_wg"\&Cstr(iwg))

result $=$ SetLinearWgAttr(oLinear, mWaveguideGeo)

Set oArc $=$ WgMgr.CreateObj("WGArc",CStr(IO_ID)\&"_Arc"\&Cstr(iwg))

result $=$ SetArcWgAttr(oArc, mWaveguideGeo)

oArc.SetAttr "RadiusExpr", CStr(Rio)

oArc.SetAttr "FixedRadius", True

$\mathrm{Jz}=\mathrm{Cz}+$ Zarc 
$\mathrm{Iz}=\mathrm{mWaferLength}$

oLinear.SetPosition $\mathrm{Iz}, \mathrm{Ix}, \mathrm{Jz}, \mathrm{Ix}$ oArc.SetPosition $\mathrm{Jz}, \mathrm{Ix}_{1} \mathrm{Cz}, \mathrm{Cx}$ Next

End Sub

End Class 'end of class Output_Array 


\section{Bibliography}

[1] D.-X. Xu, A. Delage, P. Cheben, B. Lamontagne, S. Janz, and W. N. Ye, "Siliconon-insulator (SOI) as a photonics platform," in Proc. 12 $2^{\text {th }}$ International Symp. on Silicon-on-Insulator Technology and Devices, vol. 1, 2005.

[2] G. T. Reed and A. P. Knights, Silicon Photonics: An Introduction. England: John Wiley \& Sons, Ltd., 2004.

[3] C. A. Barrios, V. R. Almeida, and M. Lipson, "Electrooptic modulation of silicon-oninsulator submicrometer-size waveguide devices," IEEE J. Lightw. Technol., vol. 21, pp. 2332-2339, 2003.

[4] C. E. Png, G. T. Reed, R. M. H. Atta, G. Ensell, and A. G. R. Evans, "Development of small silicon modulators in silicon-on-insulator (SOI)," in Proc. SPIE'03, vol. 4997, 2003, pp. 190-197.

[5] P. D. Waldron, P. E. Jessop, A. P. Yuen, L. Winchiu, P. Chyurlia, N. G. Tarr, T. J. Smy, I. Golub, D.-X. Xu, and S. Janz, "Optical modulator in silicon-on-insulator with a low thermal signature," J. Vac. Sci. Technol. A, vol. 22, pp. 800-802, 2004.

[6] V. R. Almeida, C. Barrios, R. Panepucci, and M. Lipson, "All-optical control of light on a silicon chip," Nature, vol. 431, pp. 1081-1084, 2004.

[7] P. Cheben, A. Bezinger, A. Delâge, L. Erickson, S. Janz, and D.-X. Xu, "Polarization compensation in silicon-on-insulator arrayed waveguide grating devices," in Proc. SPIE, vol. 4293, 2001, pp. 15-22.

[8] I. Day, I. Evans, A. Knights, F. Hooper, S. Roberts, J. Johnston, S. Day, J. Leff, H. Tsang, and M. Asghari, "Tapered silicon waveguides for low insertion loss highlyefficient high-speed electronic variable optical attenuators," in Proc. Optical Fiber Comm. (OFC) 2003, 2003, pp. 249-251.

[9] B. E. Little, J. S. Foresi, E. R. Thoen, G. Steinmeyer, S. T. Chu, H. A. Haus, E. P. Ippen, E. P. Kimerling, and W. Greene, "Ultra-compact $\mathrm{Si}_{-} \mathrm{SiO}_{2}$ microring resonator optical channel dropping filters," IEEE Photon. Technol. Lett., vol. 10, pp. 549-551, 1998.

[10] P. Dumon, W. Bogaerts, V. Wiaux, J. Wouters, S. Beckx, J. V. Campenhout, D. Taillaert, B. Luyssaert, P. Bienstman, D. V. Thourhout, and R. Baets, "Low-loss 
SOI photonic wires and ring resonators fabricated with deep UV lithography," IEEE Photon. Technol. Lett., vol. 16, pp. 1328-1330, 2004.

[11] V. A. Almeida, R. Panepucci, and M. Lipson, "Nanotaper for compact mode conversion," Opt. Lett., vol. 10, pp. 1302-1304, 2003.

[12] T. K. Liang and H. K. Tsang, "Integrated polarization beam splitter in high index contrast silicon-on-insulator waveguides," IEEE Photon. Technol. Lett., vol. 17, pp. 393-395, 2005.

[13] O. Boyraz and B. Jalali, "Demonstration of a silicon Raman laser," Opt. Express, vol. 12 , pp. 5269-5273, 2004.

[14] K. Iizuka, Elements of Photonics. New York: Wiley-Interscience, 2002.

[15] D.-X. Xu, W. N. Ye, A. Bogdanov, D. Dalacu, A. Delage, P. Cheben, S. Janz, B. Lamontagne, and M. J. Picard, "Design of polarization-insensitive components using geometrical and stress-induced birefringence in SOI waveguides," in Proc. SPIE, vol. 5730-19, 2005, p. 2.

[16] J. D. Plummer, M. D. Deal, and P. B. Griffin, Silicon VLSI Technology: Fundamentals, Practice, and Modeling. Prentice Hall, 2000.

[17] G. K. Celler and S. Cristoloveanu, "Frontiers of silicon-on-insulator," J. Appl. Phys., vol. 93, pp. 4955-4978, 2003.

[18] Soitec. (2006, Jan.) Innovative process. [Online]. Available: http://www.soitec.com/en/techno/t_2.htm

[19] SiGen. (2006, Jan.) Nanocleave fabrication process. [Online]. Available: http://www.sigen.com/nanocleaveprocess.htm

[20] R. A. Soref, J. Schmidtchen, and K. Petermann, "Large single-mode waveguides in GeSi-Si and Si-on-SiO 2 ," IEEE J. Quantum Electron., vol. 27, pp. 1971-1974, 1991.

[21] S. P. Pogossian, L. Vescan, and A. Vonsovici, "The single-mode condition for semiconductor rib waveguides with large cross section," IEEE J. Lightw. Technol., vol. 16, pp. 1851-1853, 1998.

[22] K. Okamoto, Fundamentals of Optical Waveguides. New York: Academic Press, 2000 .

[23] L. Pavesi and D. Lockwood, Silicon Photonics. Berlin, Germany: Springer Verlag, 2004 , ch. 10.

[24] G. P. Agrawal, Lightwave Technology: Components and Devices. New Jersey: John Wiley \& Sons, Inc., 2004. 
[25] A. G. Rickman, G. T. Reed, and F. Namavar, "Silicon-on-insulator optical rib waveguide loss and mode characters," IEEE J. Lightw. Technol., vol. 12, pp. 1771-1776, 1994.

[26] O. Powell, "Single-mode conversion for silicon rib waveguides," IEEE J. Lightw. Technol., vol. 20, pp. 1851-1855, 2002.

[27] S. P. Chan, C. E. Png, S. T. Lim, G. T. Reed, and V. M. N. Passaro, "Singlemode and polarization-independent silicon-on-insulator waveguides with small cross section," IEEE J. Lightw. Technol., vol. 23, no. 6, pp. 2103-2111, 2005.

[28] A. Tarraf, J. Daleiden, S. Irmer, D. Prasai, and H. Hillmer, "Stress investigation of PECVD dielectric layers for advanced optical MEMS," J. Micromech. Microeng., vol. 14, pp. 317-323, 2004.

[29] A. Yariv and P. Yeh, Optical Waves in Crystals. New Jersey: Wiley-Interscience, 2003.

[30] L. Vivien, S. Laval, B. Dumont, S. Lardenois, A. Koster, and E. Cassan, "Polarization-independent single-mode rib waveguides on silicon-on-insulator for telecommunication wavelengths," Optics Comm., vol. 210, pp. 43-49, 2002.

[31] M. R. T. Pearson, A. Bezinger, A. Delâge, J. W. Fraser, S. Janz, P. E. Jessop, and D.-X. Xu, "Arrayed waveguide grating demultiplexers in silicon-on-insulator," in Proc. SPIE'99, vol. 3593, 2000, pp. 11-18.

[32] D.-X. Xu, P. Cheben, D. Dalacu, S. Janz, M.-J. Picard, N. G. Tarr, and W. N. Ye, "Control and compensation of birefringence in SOI waveguides," in Proc. $16^{\text {th }}$ Annual Meeting of the IEEE Lasers and Electro-Optics Society, LEOS'03, 2003, pp. 530-531.

[33] S. Janz, A. Balakrishnan, S. Charbonneau, P. Cheben, M. Cloutier, A. Delage, K. Dossou, L. Erickson, M. Gao, P. A. Krug, B. Lamontagne, M. Packirisamy, M. Pearson, and D.-X. Xu, "Planar waveguide echelle gratings in silica-on-silicon," IEEE Photon. Technol. Lett., vol. 16, pp. 503-505, 2004.

[34] S. Suzuki, Y. Inoue, and Y. Ohmori, "Polarisation insensitive arrayed-waveguide grating multiplexer with $\mathrm{SiO}_{2}$-on-SiO 2 structure," Electron. Lett., vol. 30, pp. 642643, 1994.

[35] B. Buchold and E. Voges, "Polarisation insensitive arrayedwaveguide grating multiplexers with ion-exchanged waveguides in glass," Electron. Lett., vol. 32, pp. 2248$2250,1996$.

[36] P. Ayras, G. N. Conti, S. Honkanen, and N. Peyghambarian, "Birefringence control for ion-exchanged channel glass waveguides," Appl. Opt., vol. 37, pp. 8400-8405, 1998. 
[37] S. Suzuki, S. Sumida, Y. Inoue, M. Ishii, and Y. Ohmori, "Polarisation insensitive arrayed-waveguide gratings using dopant-rich silica-based glass with thermal expansion adjusted to Si substrate," Electron. Lett., vol. 33, pp. 1173-1179, 1997.

[38] S. M. Ojha, C. Cureton, T. Bricheno, S. Day, D. Moule, A. J. Bell, and J. Taylor, "Simple method of fabricating polarisation insensitive and very low crosstalk AWG grating devices," Electron. Lett., vol. 34, pp. 78-79, 1998.

[39] J. Canning, "Birefringence control in planar waveguides using doped top layers," Optics Comm., vol. 191, pp. 225-228, 2001.

[40] A. Kilian, J. Kirchhof, B. Kuhlow, G. Przyrembel, and W. Wischmann, "Birefringence free planar optical waveguide made by flame hydrolysis deposition (FHD) through tailoring of the overcladding," IEEE J. Lightw. Technol., vol. 18, pp. 193$198,2000$.

[41] H. Takahashi, Y. Hibino, and I. Nishi, "Polarization-insensitive arrayed-waveguide grating wavelength multiplexer on silicon," Opt. Lett., vol. 17, pp. 499-501, 1992.

[42] M. Okuno, A. Sugita, K. Jinguji, and M. Kawachi, "Birefringence control of silica waveguides on $\mathrm{Si}$ and its application to a polarization-beam splitter/switch," IEEE J. Lightw. Technol., vol. 12, pp. 625-633, 1994.

[43] H. Takahashi, Y. Hibino, Y. Ohmori, and M. Kawachi, "Polarization-insensitive arrayed-waveguide wavelength multiplexer with birefringence compensating film," IEEE Photon. Technol. Lett., vol. 5, pp. 707-709, 1993.

[44] E. K. Wildermuth, C. K. Nadler, M. Lanker, W. Hunziker, and H. Melchior, "Penalty-free polarisation compensation of $\mathrm{SiO}_{2} / \mathrm{Si}$ arrayed waveguide grating wavelength multiplexers using stress release grooves," Electron. Lett., vol. 34, pp. 16611663, 1998.

[45] C. K. Nadler, E. K. Wildermuth, M. Lanker, W. Hunziker, and H. Melchior, "Polarization insensitive, low-loss, low-crosstalk wavelength multiplexer modules," IEEE J. Select. Top. Quantum Electron., vol. 5, pp. 1407-1412, 1999.

[46] J.-J. He, E. S. Kosteles, B. Lamontagne, L. Erickson, A. Delage, and M. Davis, "Integrated polarization compensator for WDM waveguide demultiplexers," IEEE Photon. Technol. Lett., vol. 11, pp. 224-226, 1999.

[47] J. Albert, F. Bilodeau, D. C. Johnson, K. O. Hill, S. J. Milhailov, D. Stryckman, T. Kitagawa, and Y. Hibino, "Polarization-independent strong Bragg gratings in planar lightwave circuits," Electron. Lett., vol. 34, pp. 485-486, 1998.

[48] J. Canning, M. Aslund, A. Ankiewicz, M. Dainese, H. Fernando, J. K. Sahu, and L. Wosinski, "Birefringence control in plasma-enhanced chemical vapor deposition planar waveguides by ultraviolet irradiation," Appl. Opt., vol. 39, pp. 4296-4299, 2000 . 
[49] X. Dai, S. J. Milhailov, C. Blanchetiere, C. L. Callender, and R. B. Walker, "High birefringence control and polarization insensitive Bragg grating fabricated in PECVD planar waveguide with UV polarized irradiation," Optics Comm., vol. 248, pp. 123-130, 2005.

[50] J. Canning and M. Aslund, "Birefringence compensation, improved fringe contrast and trimming in an integrated asymmetric Mach-Zehnder interferometer using midir laser processing," Opt. Mat., vol. 14, pp. 175-183, 2000.

[51] J. Albert, F. Bilodeau, D. C. Johnson, K. O. Hill, K. Hattori, T. Kitagawa, Y. Hibino, and M. Abe, "Low-loss planar lightwave circuit oadm with high isolation and no polarization dependence," IEEE Photon. Technol. Lett., vol. 11, pp. 346-348, 1999.

[52] H. Bissessur, F. Gaborit, B. Martin, P. Pagnod-Rossiaux, J.-L. Peyre, and M. Renaud, "16 channel phased array wavelength demultiplexer on InP with low polarisation sensitivity," Electron. Lett., vol. 30, pp. 336-337, 1994.

[53] H. Bissessur, P. Pagnod-Rossiaux, M. Renaud, and B. Martin, "Extremely small polarization independent phased-array demultiplexer on InP," IEEE Photon. Technol. Lett., vol. 8, pp. 554-556, 1996.

[54] L. G. de Peralta, A. A. Bernussi, H. Temkin, M. M. Borhani, and D. E. Doucette, "Silcon-dioxide waveguides with low birefringence," IEEE J. Select. Top. Quantum Electron., vol. 39, pp. 874-879, 2003.

[55] J. B. D. Soole, M. R. Amersfoort, H. P. LeBanc, N. C. Andreadakis, A. Rajhel, C. Caneau, M. A. Koza, R. Bhat, C. Youtsey, and I. Adesida, "Polarisationindependent InP arrayed waveguide filter using square cross-section waveguides," Electron. Lett., vol. 32, pp. 323-324, 1996.

[56] M. Zirngibl, C. H. Joyner, L. W. Stulz, T. Gaiffe, and C. Dragone, "Polarisation independent $8 \times 8$ waveguide grating multiplexer on InP," Electron. Lett., vol. 29, pp. 201-202, 1993.

[57] M. Zirngibl, C. H. Joyner, and P. C. Chou, "Polarisation compensated waveguide grating on InP," Electron. Lett., vol. 31, pp. 1662-1664, 1995.

[58] E. Gini, W. Hunziker, and H. Melchior, "Polarization independent WDM multiplexer/demultiplexer module," IEEE J. Lightw. Technol., vol. 16, pp. 625-630, 1998.

[59] E. Cassan, L. Vivien, and S. Laval, "Polarization-independent $90^{\circ}$-turns in singlemode micro-waveguides on silicon-on-insulator wafers for telecommunication wavelengths," Optics Comm., vol. 235, pp. 83-88, 2004.

[60] T. Baba, "Si photonic wire waveguides," in Proc. SPIE'04, vol. 5515, 2004, pp. 150-157. 
[61] K. Sasaki, F. Ohno, A. Motegi, and T. Baba, "Arrayed waveguide grating of $70 \times$ $60 \mu \mathrm{m}$ size based on Si photonic wire waveguides," Electron. Lett., vol. 41, pp. $801-802,2005$.

[62] D. Dai and S. He, "Design of a polarization-insensitive arrayed waveguide grating demultiplexer based on silicon photonic wires," Opt. Lett., vol. 31, pp. 1988-1990, 2006 .

[63] D. Dai and S. He, "Optimization of ultracompact polarization insensitive multimode interference couplers based on Si nanowire waveguides," IEEE Photon. Technol. Lett., vol. 18, pp. 2017-2019, 2006.

[64] D. Dai and S. He, "Analysis of the birefringence of silicon-on-insulator rib waveguide," Appl. Opt., vol. 43, pp. 1156-1161, 2004.

[65] P. D. Trinh, S. Yegnanarayanan, F. Coppinger, and B. Jalali, "Silicon-on-insulator (SOI) phased-array wavelength mulit/demultiplexer with extremely low-polarization sensitivity," IEEE Photon. Technol. Lett., vol. 9, pp. 940-942, 1997.

[66] P. Cheben, D.-X. Xu, S. Janz, A. Delâge, and D. Dalacu, "Birefringence compensation in silicon-on-insulator planar waveguide demultiplexers using a buried oxide layer," in Proc. SPIE, vol. 4997, 2003, pp. 181-189.

[67] W. R. Headley, G. T. Reed, S. Howe, A. Liu, and M. Paniccia, "Polarizationindependent optical racetrack resonators using rib waveguides on silicon-oninsulator," Appl. Phys. Lett., vol. 85, pp. 5523-5525, 2004.

[68] S. W. Roberts, "Controlling birefringence in an optical waveguide and in an arrayed waveguide grating," Bookham Technology, Great Britain Patent WO02/27366, April $4,2002$.

[69] W. N. Ye, D.-X. Xu, S. Janz, P. Cheben, A. Delâge, M. J. Picard, B. Lamontagne, and N. G. Tarr, "Stress-induced effects on birefringence for silion-on-insultor (SOI) waveguides," in Proc. SPIE, vol. 5357, 2004, pp. 57-66.

[70] D.-X. Xu, S. Janz, and P. Cheben, "Design of polarization-insensitive ring resonators in silicon-on-insulator using MMI couplers and cladding stress engineering," IEEE Photon. Technol. Lett., vol. 18, pp. 343-345, 2006.

[71] D.-X. Xu, P. Cheben, A. Delage, S. Janz, B. Lamontagne, M.-J. Picard, E. Post, P. Waldron, and W. N. Ye, "Applications of cladding stress induced effects for advanced polarization control in silicon photonics," in Proc. Progress In Electromagnetics Research Symposium (PIERS'07), Mar. 2007 (to be published).

[72] S. Janz, J. Ctyroky, and S. Tanev, Frontiers in Planar Lightwave Circuit Technology: Design, Simulation, and Fabrication. Dordrecht, The Netherlands: Springer Verlag, 2006, ch. 1 . 
[73] A. R. Vellekoop and M. K. Smit, "A small-size polarization splitter based on a planar optical phase array," IEEE J. Lightw. Technol, vol. 8, pp. 118-124, 1990.

[74] O. Mikami, "LiNbO ${ }_{3}$ coupled-waveguided TE/TM mode splitter," Appl. Phys. Lett., vol. 36, pp. 491-492, 1980.

[75] G. Kim, B. Kang, S. Lee, H. Chang, M. Choi, S. Lee, D. Woo, and S. Kim, "A multimode-interferenced electrooptic TE/TM mode splitter," in Proc.1999 CLEOPacific Rim, vol. 2, 1999.

[76] P.-K. Wei and W.-S. Wang, "A TE-TM mode splitter on lithium niobate using Ti, $\mathrm{Ni}$, and MgO diffusions," IEEE Photon. Technol. Lett., vol. 6, pp. 245--248, 1994.

[77] Y.-P. Liao, R.-C. Lu, C.-H. Yang, and W.-S. Wang, "Passive Ni:LiNbO 3 polarization splitter at $1.3 \mu \mathrm{m}$ wavelength," Electron. Lett., vol. 32, pp. 1003-1004, 1996.

[78] W.-H. Hsu, K.-C. Lin, J.-Y. Li, W. Y.-S, and W.-S. Wang, "Polarization splitter with variable TE-TM mode converter using $\mathrm{Zn}$ and $\mathrm{Ni}$ codiffused $\mathrm{LiNbO}_{3}$ waveguides," IEEE J. Lightw. Technol., vol. 11, pp. 271-277, 2005.

[79] L. B. Soldano, A. H. de Vreede, M. K. Smit, B. H. Verbeek, E. G. Metaal, and F. H. Groen, "Mach-Zehnder interferometer polarization splitter in InGaAsP/InP," IEEE Photon. Technol. Lett., vol. 6, pp. 402-405, 1994.

[80] P. Albrecht, M. Hamacher, H. Heidrich, D. Hoffmann, H.-P. Nolting, and C. M. Weinert, "TE/TM mode splitter on InGaAsP/InP," IEEE Photon. Technol. Lett., vol. 2, pp. 114-115, 1990.

[81] A. A. I. Kiyat and N. Dagli, "A compact silicon-on-insulator polarization splitter," IEEE Photon. Technol. Lett., vol. 17, pp. 100-102, 2005.

[82] R. A. Betts and F. Lui, "Broadband polarization splitting couoplers in ion-exchange glass," Electron. Lett., vol. 26, pp. 450-452, 1990.

[83] M. Huang, "Stress effects on the performance of optical waveguides," Inter. J. Solids and Structures, vol. 40, pp. 1615-1632, 2003.

[84] K.-H. Hellwege and A. Hellwege, Eds., Landolt-Bornstein: Numerical Data and Functional Relationships in Science and Technology: New Series., ser. Group III, Crystal and Solid State Physics. Berlin, Germany: Springer Verlag, 1979, vol. 11.

[85] S. P. Timoshenko and J. N. Goodier, Theory of Elasticity. New York: McGraw-Hill, 1970.

[86] K. Saitoh, M. Koshiba, and Y. Tsuji, "Stress analysis method for elastically anisotropic material based optical waveguides and its application to strain-induced optical waveguides," IEEE J. Lightw. Technol., vol. 17, pp. 255--259, 1999.

[87] X.-Z. Zhao, Y.-Z. Xu, and C. Li, "Birefringence control in optical planar waveguides," IEEE J. Lightw. Technol., vol. 21, pp. 2352-2357, 2003. 
[88] K. Röll, "Analysis of stress and strain distribution in thin films and substrates," $J$. Appl. Phys., vol. 47, pp. 3224-3229, 1976.

[89] D.-X. Xu, P. Cheben, D. Dalacu, A. Delâge, S. Janz, B. Lamontagne, M. Picard, and W. N. Ye, "Eliminating the birefringence in silicon-on-insulator ridge waveguides using the cladding stress," Opt. Lett., vol. 29, no. 20, pp. 2384-2386, 2004.

[90] D.-X. Xu, J.-M. Baribeau, P. Cheben, D. Dalacu, A. Delâge, B. Lamontagne, S. Janz, M. Picard, W. N. Ye, and N. G. Tarr, "Prospects and challenges for microphotonic waveguide components based on Si and SiGe," in Proc. Electrochemical Society Fall Meeting (ECS'04), vol. 1, 2004.

[91] T.-H. Kim, H.-K. Sung, J.-W. Choi, and K.-H. Yoon, "Effective silicon oxide formation on silica-on-silicon platforms for optical hybrid integration," Electron. Telecom. Res. Inst. (ETRI) Journal, vol. 25, pp. 74-80, 2003.

[92] D.-X. Xu, P. Cheben, S. Janz, and D. Dalacu, "Control of SOI waveguide polarization properties for microphotonic applications," in Proc. $5^{\text {th }}$ CLEO-Pacific Rim, vol. 1, 2003.

[93] L. B. Soldano and E. C. M. Pennings, "Optical multi-mode interference devices based on self-imaging: principles and applications," IEEE J. Lightw. Technol., vol. 13, pp. 615-627, 1995.

[94] M. K. Smit and C. van Dam, "PHASAR-based WDM-devices: principles, design and applications," IEEE J. Select. Top. Quantum Electron., vol. 2, pp. 236-250, 1996.

[95] E. Fluck, F. Horst, B. J. Offrein, R. Germann, H. W. M. Salemink, and G.-L. Bona, "Compact versatile thermooptical space switch based on beam steering by a waveguide array," IEEE Photon. Technol. Lett., vol. 11, pp. 1399-1401, 1999. 